\title{
Uma introdução à Lógica de Segunda Ordem
}

Enéas Alves Nogueira Júnior

DisSERTAÇÃO APRESENTADA

$\mathrm{AO}$

Instituto DE MATEMÁtica E EstatísticA

DA

Universidade DE SÃo PAUlo

PARA

OBTENÇÃO DO TÍTULO

$\mathrm{DE}$

Mestre EM CiÊNCIAS

Programa: Matemática

Orientador: Prof. Dr. Francisco Miraglia Neto

Durante o desenvolvimento deste trabalho o autor recebeu auxílio financeiro do CNPq

São Paulo, fevereiro de 2013 


\title{
Uma Introdução à Lógica de Segunda Ordem
}

\begin{abstract}
Esta versão da dissertação contém as correções e alterações sugeridas pela Comissão Julgadora durante a defesa da versão original do trabalho, realizada em 26/04/2013. Uma cópia da versão original está disponível no Instituto de Matemática e Estatística da Universidade de São Paulo.
\end{abstract}

Comissão Julgadora:

- Prof. Dr. Francisco Miraglia Neto (orientador) - IME-USP

- Prof. Dr. Ricardo Bianconi - IME-USP

- Prof. Dr. Walter Alexandre Carnielli - UNICAMP 


\title{
Agradecimentos
}

\author{
A lista é longa.
}

Em primeiro lugar, eu agradeço a todos que tiveram um envolvimento mais direto com o desenvolvimento deste trabalho: meu orientador, o Prof. Dr. Francisco Miraglia Neto, e a minha família, que me aturou a maior parte do tempo: meus pais Enéas e Elaine e meu irmão Bruno. Também agradeço a Deus aqui; eu e Ele temos nossas diferenças, mas o reconheço como provedor de tudo.

Em segundo lugar, agradeço a todos os meus amigos que me ouviram quando eu reclamava da vida e dizia que não aguentava mais trabalhar neste texto, com destaque para o Mauricio HCS, o Tiago Mendonça e o Rodolfo Marinho.

Em terceiro lugar, eu gostaria de citar alguns amigos que não me acompanharam o tempo todo, mas que foram significativos em diversos momentos: Patrícia Nascimento, Caio Hornhardt, Helen Samara, Carolina Alexiou, Antonieta Pierroti, Gabriel Zanetti, Anderson Almeida, André Ferreira, Eric Araújo, Lucas Torres, Elaine Torres, Karina Silva, Cinthia Campos, Claudia Correia, Rodrigo Freire e o Prof. Dr. Hugo Mariano. Sem a ajuda e as conversas que eu joguei fora com vocês, minha vida teria sido muito chata e mais difícil, obrigado por tudo.

Em quarto lugar, eu agradeço aos professores do IME-USP que eu tive o privilégio de ser aluno.

Em quinto lugar, eu agradeço ao CNPq pelo auxílio financeiro, e à comissão do IME-USP que me possibilitou o acesso à esta bolsa. 


\section{Resumo}

Neste trabalho investigamos alguns aspectos da Lógica de Segunda Ordem, dividindo o tema em três capítulos. No primeiro capítulo discorremos sobre os conceitos básicos desta Lógica, tais como conjunto de fórmulas, sistemas dedutivos e semânticas. Fazemos também um contraste com a Lógica de Primeira Ordem, que é mais conhecida, para se ter uma espécie de modelo do qual estamos nos diferenciando. Provamos o teorema da completude para a Lógica de Segunda Ordem, devido a L. Henkin em Henkin (1950).

No segundo capítulo nós procuramos entender o que acontece com a semântica da teoria de conjuntos $Z F C$ (que é de primeira ordem) se adicionarmos alguns axiomas de segunda ordem, criando uma teoria que chamamos de $Z F 2$. Mostramos um teorema devido a Zermelo (Zermelo (1930)) que diz que os modelos desta teoria são essencialmente os mesmos. Também procuramos investigar a questão da Hipótese do Contínuo com relação à $Z F 2$ e, através de um metódo de forcing para esta teoria, mostramos que a $H C$ continua sem resposta.

No terceiro capítulo, escrevemos sobre três temas diferentes: o primeiro é sobre a relação que existe entre a propriedade da completude, da compacidade e a semântica de Henkin. O teorema de Lindström, que provamos nesta seção, diz essencialmente que não podemos ter completude e compacidade para a Lógica de Segunda Ordem ao menos que usemos esta semântica. Na segunda seção, investigamos o número de Hanf da Lógica de Segunda Ordem com a semântica Padrão e, na terceira seção, mostramos que é possível fazer uma redução das Lógicas de ordem superior à segunda e que o conjunto das fórmulas válidas da Lógica de Segunda Ordem não é definível na estrutura dos números naturais.

Palavras-chave: Lógica de Segunda Ordem, Lógica de Ordem Superior, Teoria de Tipos. 


\section{Abstract}

In this work we investigate some aspects of Second-Order Logic, splitting the theme in three chapters. In the first one, we discuss the basic concepts of that Logic, such as set of formulas, deductive systems and semantics. We also make a contrast with First-Order Logic, which is better know, in order to have some kind of model from wich we are differentiating. We prove the theorem of the completeness for the Second-Order Logic, due to L. Henkin in Henkin (1950).

In the second chapter we try to understand what happens with the semantics of the $Z F C$ set theory (which is a First-Order theory) if we add some Second-Order axioms, creating a theory that we call $Z F 2$. We prove a theorem due to Zermelo (Zermelo (1930)) which says that the models of this theory are essentially the same. We also investigate the question of the Continuum Hypothesis in relation to $Z F 2$ and, through a method of forcing for that theory, we show that the $H C$ still has no answer.

In the third chapter, we write about three different themes: the first is about the relation that exists between the property of completeness, of compactness and the Henkin semantics. The Lindström's theorem, which we prove in this section, says essentially that we can't have the completeness and the compactness for the Secon-Order Logic without Henkin semantics. In the second section, we investigate the Hanf Number of Second-Order Logic and, in the third section, we show that it is possible to make a reduction of Logics of order higher than the second to the second and that the set of the Second-Order valid formulas is not definable in the structure of the natural numbers.

Keywords: Second-Order Logic, Higher-Order Logic, Type Theory. 


\section{Sumário}

Lista de Símbolos $\quad$ ix

1 Primeira Ordem versus Segunda Ordem 1

1.1 Introdução . . . . . . . . . . . . . . . . . . . . . . . 1

1.2 Sintaxe e Semântica Padrão de L2 . . . . . . . . . . . . . . . . 7

1.3 Semântica de Henkin . . . . . . . . . . . . . . . . . . . . . . . 14

1.4 Sistemas Dedutivos e L $\ldots \ldots \ldots \ldots \ldots$. . . . . . . . . . . . 21

2 Segunda Ordem e Teoria de Conjuntos 25

2.1 ZF2 e sua consistência com a Hipótese do Contínuo . . . . . . . . . . . 25

2.2 Forcing em ZF2 . . . . . . . . . . . . . . . . . . . . . . 32

$2.3 \mathrm{Um}$ modelo de $\mathrm{ZF} 2+\neg \mathrm{HC} \ldots \ldots \ldots \ldots \ldots$. . . . . . . . . . . . . . .

2.4 Importando resultados de consistência com ZFC . . . . . . . . . . . . . 47

2.5 Forcing para Classes . . . . . . . . . . . . . . . . 50

2.6 Cardinais em $\mathrm{M}[\mathrm{G}]$ para $\mathrm{G}$ fortemente genérico $\ldots \ldots \ldots \ldots$

2.7 A independência da HGC e o teorema de Easton . . . . . . . . . . . . . 58

3 Outros Tópicos $\quad 63$

3.1 O Teorema de Lindström . . . . . . . . . . . . . . . . . . 63

3.2 O Número de Hanf de $L 2 \ldots \ldots$. . . . . . . . . . . . . . . . . 66

3.3 Definição de Verdade e Reduções . . . . . . . . . . . . . . . . 71

4 Considerações Finais $\quad 79$

$\begin{array}{ll}\text { Referências Bibliográficas } & 81\end{array}$ 
viii SUMÁRIO 


\section{Lista de Símbolos}

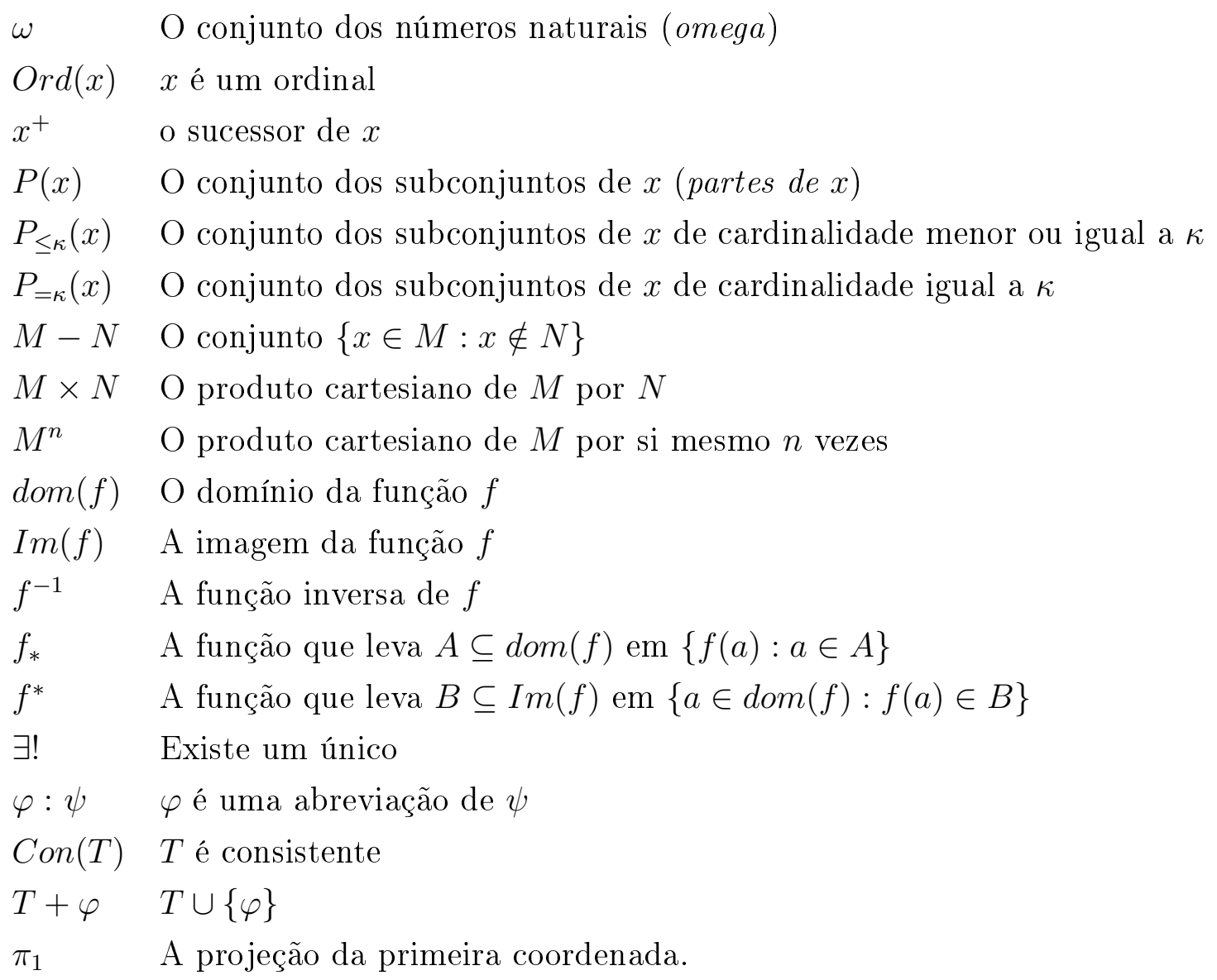




\section{Capítulo 1}

\section{Primeira Ordem versus Segunda Ordem}

\subsection{Introdução. Lógica Abstrata e Lógica de Primeira Ordem}

Uma linguagem (ou lógica) de Segunda Ordem é uma extensão de uma de Primeira Ordem; a diferença básica entre as duas é a de que em Segunda Ordem permitimos quantificação não apenas sobre os objetos do discurso, mas também sobre coleções, ou propriedades destes objetos. É claro que poderíamos também, se quiséssemos, permitir quantificação sobre coleções de coleções; neste caso teríamos uma linguagem de Terceira Ordem. Essa construção pode ser extendida até, digamos, a $n$-ésima ordem ou ainda indefinidamente, com todas as ordens; tais linguagens, junto com a de Segunda Ordem, são chamadas de linguagens de Ordem Superior; elas não são o foco do nosso trabalho, mas iremos estudá-las um pouco, como uma generalização natural deste segundo nível da linguagem que estaremos estudando.

O que nos motivou a este estudo foi, basicamente, uma curiosidade sobre o comportamento da linguagem quando deixávamos a Primeira e partíamos para a Segunda Ordem. Qualquer um que tome em mãos um livro moderno de introdução à Lógica poderá ver muito espaço dedicado à Lógica de Primeira Ordem e quase nenhum à de Segunda, se algum. E isso a despeito de ser uma extensão muito natural, a nosso ver. Na verdade, olhando históricamente, vemos que nos trabalhos do final do século XIX e no começo do XX as linguagens usadas eram, a grosso modo, de Ordem Superior (Löwenheim, em 1915, foi o primeiro a fazer uma distinção clara entre Lógica de Primeira e de Segunda Ordens, veja Moore (1980)); essa atenção especial para a Lógica de Primeira Ordem se deu anos mais tarde, por conta de que descobriu-se que esse fragmento desfruta de muitas meta-propriedades interessantes e relativamente fáceis de ser manejadas, o que não é o caso das linguagens de ordem superior (se entendermos sua noção de modelo do modo "standard"; isto deve ficar claro ao longo do texto).

Lógicas de Ordem Superior foram não apenas deixadas de lado por muito tempo, mas até mesmo sofreram "ataques" por parte de alguns estudiosos, por conta de suas pressuposições ontológicas. Acontece que dado haver essa quantificação sobre coleções de objetos, muitas propriedades que normalmente se espera depender apenas da linguagem acabam por depender da interpretação da teoria de conjuntos que modela a semântica da linguagem. Por conta disso, chegou-se até mesmo a dizer que tais linguagens não deviam nem ao menos poder se chamarem de "Lógicas"; nas palavras de W. O. Quine, "Lógica de Segunda Ordem é Teoria de Conjuntos em pele de cordeiro".

Nesta seção, queremos introduzir algumas noções de Lógica Abstrata, bem como noções sintáticas e semânticas da Lógica de Primeira Ordem, com o objetivo de estabelecer notação 
e tornar mais preciso o discurso que irá seguir.

Definição 1.1.1 (a) Um vocabulário $V$ é uma coleção qualquer, onde seus elementos são divididos de maneira disjunta em simbolos de constante, símbolos de funções e símbolos de relações. A cada símbolo de função e de relação está associado um inteiro positivo chamado de aridade do símbolo. Escrevemos $V=C \cup F \cup R$ para indicar $V$ como a união dos diversos símbolos, de tal forma que se $v \in C$, então $v$ é símbolo de constante, se $v \in F$, então $v$ é símbolo de função, e se $v \in R$, então $v$ é símbolo de relação. Também escrevemos $F=\bigcup_{n \geq 1} F_{n}$ e $R=\bigcup_{n \geq 1} R_{n}$, de tal forma que se $v \in F_{n} \cup R_{n}$, então $v$ é um símbolo $n$-ário.

(b) Uma estrutura de vocabulário $V$, ou uma $V$-estrutura, é um par $M=\langle|M|, I\rangle$, onde $|M|$ é um conjunto não vazio e I é uma função de dominio $V$. I é chamada de função de interpretação e associa, a cada simbolo de constante $c \in V$, um elemento $I(c) \in|M|$, e a cada símbolo de função $n$-ária $f \in V$, uma função $I(f):|M|^{n} \rightarrow|M|$, e a cada símbolo de relação $n$-ária $P \in V$ uma relação $n$-ária $I(P) \subseteq|M|^{n}$. Frequentemente escreve-se $c^{M}$ no lugar de $I(c), f^{M}$ no lugar de $I(f)$, etc. O vocabulário de uma estrutura $M$ indica-se por $V_{M}$. Como de praxe, iremos usar $M$ para indicar tanto o conjunto $|M|$ quanto a $V$-estrutura $\langle|M|, I\rangle$, se não houver perigo de confusão. Também escreveremos $\left\langle|M|, c^{M}, f^{M}, P^{M}\right\rangle$, por exemplo, para indicar a estrutura cuja função de interpretação I é tal que $I(c)=c^{M}, I(f)=f^{M}$, etc.

(c) Um isomorfismo entre $V$-estruturas $M$ e $M^{\prime}$ é uma função bijetora $F: M \rightarrow M^{\prime}$ tal que:

(i) para cada simbolo de constante $c \in V, F\left(c^{M}\right)=c^{M^{\prime}}$;

(ii) para cada símbolo de função n-ária $f \in V$, para todos $a_{1}, \ldots, a_{n} \in M, F\left(f^{M}\left(a_{1}, \ldots, a_{n}\right)\right)$ $=f^{M^{\prime}}\left(F\left(a_{1}\right), \ldots, F\left(a_{n}\right)\right)$, e

(iii) para cada símbolo de relação $n$-ária $P \in V$, para todos $a_{1}, \ldots, a_{n} \in M,\left\langle a_{1}, \ldots, a_{n}\right\rangle \in$ $P^{M} \Leftrightarrow\left\langle F\left(a_{1}\right), \ldots, F\left(a_{n}\right)\right\rangle \in P^{M^{\prime}}$.

Escrevemos $F: M \cong M^{\prime}$ para indicar que $F: M \rightarrow M^{\prime}$ é um isomorfismo entre $M$ e $M^{\prime}$.

(d) $S e V^{\prime} \subseteq V$ e $M$ é uma $V$-estrutura, então definimos o reduto de $M$ a $V^{\prime}, M \uparrow V^{\prime}$, como sendo a $V^{\prime}$-estrutura formada pelo conjunto $M$ e cuja função de interpretação $I^{\prime}$ é a função de interpretação I de $M$ restrita a $V^{\prime}$.

(e) Uma função $\rho: V^{\prime} \rightarrow V$ é chamada de um renomeamento de $V^{\prime}$ em $V$ se for uma bijeção que leva símbolos de constante em símbolos de constante, símbolos de função n-ária em símbolos de função n-ária e símbolos de relação n-ária em símbolos de relação n-ária. Dada uma $V$-estrutura $M$, indicamos por $M^{\rho}$ a estrutura $\langle M, I \circ \rho\rangle$ obtida por renomear-se os elementos de $V$.

Definição 1.1.2 Uma lógica é um par $\left\langle L, \vDash_{L}\right\rangle$, onde $L$ é uma função definida para cada vocabulário $V$ tal que $L(V)$ é uma classe, chamada de classe das $L$-sentenças de vocabulário $V, e \vDash_{L}$ é uma relação entre $V$-estruturas e L-sentenças, chamada de relação de $L$-satisfação, verificando as seguintes propriedades:

(i) $S$ e $V^{\prime} \subseteq V$, então $L\left(V^{\prime}\right) \subseteq L(V)$;

(ii) $S$ e $M \vDash_{L} \varphi$, entãa $\varphi \in L\left(V_{M}\right)$;

(iii) Propriedade do Isomorfismo. Se $M \vDash_{L} \varphi$ e $M \cong N$, então $N \vDash_{L} \varphi$;

(iv) Propriedade do Reduto. Se $\varphi \in L\left(V^{\prime}\right)$ e $V^{\prime} \subseteq V$, então

$$
M \vDash_{L} \varphi \Leftrightarrow M \uparrow V^{\prime} \vDash_{L} \varphi \text {. }
$$

(v) Propriedade do renomeamento. Seja $\rho: V^{\prime} \rightarrow V$ um renomeamento. Então, para cada $\varphi \in L(V)$ existe uma sentença $\varphi^{\rho} \in L\left(V^{\prime}\right)$ tal que para todas as $V$-estruturas $M$,

$$
M \vDash_{L} \varphi \Leftrightarrow M^{\rho} \vDash_{L} \varphi^{\rho} .
$$


Aqui também, como de costume, escrevemos simplesmente $L$ no lugar de $\left\langle L, \vDash_{L}\right\rangle, e \vDash n o$ lugar de $\vDash_{L}$, desde que não haja perigo de confusão.

Nós agora começaremos a definir o que seria a Lógica de Primeira Ordem. Faltam ainda várias definições necessárias e que são gerais o suficiente para qualquer Lógica que iremos considerar neste trabalho (como, por exemplo, equivalência de estruturas, ou a noção de consequencia semântica), mas vamos deixar para introduzí-las conforme for necessário.

Definição 1.1.3 O alfabeto da linguagem de primeira ordem com vocabulário $V$, Alf $f_{V}$, é o conjunto formado pela união disjunta dos seguintes conjuntos:

(a) um vocabulário $V$;

(b) um conjunto Var de variáveis (de primeira ordem);

(c) um conjunto de símbolos lógicos $\{\neg($ não), $\rightarrow$ (implica), $\forall$ (para todo) $\}$;

(d) um conjunto de simbolos linguísticos $\{()$, ,", $\}$ (isto é, parêntesis à esquerda e à direita e vírgula).

Vemos assim que o conjunto $V \cup \operatorname{Var} \cup\{\neg, \rightarrow, \forall\} \cup\{($,$) , "," \}$ é um alfabeto para uma linguagem de primeira ordem. Assumiremos daqui para frente que Var é um conjunto infinito enumerável $\operatorname{Var}=\left\{x_{1}, x_{2}, \ldots\right\}$ e, como sempre, subscritos serão omitidos, se não houver perigo de confusão.

Definição 1.1.4 A linguagem de primeira ordem com vocabulário $V$ é a tripla $\left\langle A l f_{V}\right.$, Term, Form $\rangle$, onde $A l f_{V}$ é o alfabeto da linguagem de primeira ordem com vocabulário $V$ e Term e Form são conjuntos, chamados respectivamente de conjuntos dos termos e das fórmulas. Eles são construídos recursivamente segundo as condições (a) e (b) a seguir:

(a) Term é um conjunto tal que $t \in$ Term se, e somente se, existe uma sequencia $t_{1}, \ldots, t_{n}$ onde $_{n}$ é $t$ e, para cada $i, 1 \leq i \leq n$,

(i) $t_{i}$ é uma variável, ou

(ii) $t_{i}$ é símbolo de constante de $V$, ou

(iii) $t_{i}$ é da forma $f\left(t_{i_{1}}, \ldots, t_{i_{m}}\right),{ }^{1}$ onde $f$ é um símbolo de função m-ária e $i_{1}, \ldots, i_{m}<$ $i$.

A complexidade $c(t)$ de um termo $t$ é o menor $n$ para o qual existe uma sequencia como acima.

(b) Form é um conjunto tal que $\varphi \in$ Form se, e somente se, existe uma sequencia $\varphi_{1}, \ldots, \varphi_{n}$ onde $\varphi_{n}$ é $\varphi$ e, para cada $i, 1 \leq i \leq n$,

(i) $\varphi_{i}$ é da forma $P\left(t_{1}, \ldots, t_{m}\right)$, com $P \in V$ um símbolo de relação $m$-ária $e$ $t_{1}, \ldots, t_{m} \in$ Term (tais elementos de Form são chamados de fórmulas atômicas), ou

(ii) $\varphi_{i}$ é da forma $\neg \varphi_{j}$ ou $\varphi_{j} \rightarrow \varphi_{k}$, com $j, k<i$, ou

(iii) $\varphi_{i}$ é da forma $\forall x \varphi_{j}$, com $x \in \operatorname{Var} e j<i$.

$N a$ fórmula em (iii), $\varphi_{j}$ recebe o nome de escopo do quantificador $\forall$, e qualquer ocorrência da variável $x$ em $\forall x \varphi_{j}$ é dita ligada; ocorrências de $x$ que não são ligadas são ditas livres. Uma sentença é uma fórmula sem ocorrência de variáveis livres. A complexidade $c(\varphi)$ de $\varphi$ é o menor n para o qual existe uma sequencia como acima.

Introduzimos os conectivos usuais restantes por abreviação: $\varphi \vee \psi:(\neg \varphi) \rightarrow \psi, \varphi \wedge \psi$ : $\neg(\neg \varphi \vee \neg \psi), \varphi \leftrightarrow \psi:(\varphi \rightarrow \psi) \wedge(\psi \rightarrow \varphi) e \exists x \varphi: \neg \forall x \neg \varphi$.

Passaremos agora a descrever o sistema dedutivo da Lógica de Primeira Ordem. Nosso estudo focará a Lógica Clássica, e portanto as definições seguintes são padrão.

\footnotetext{
${ }^{1}$ Quando dizemos que $t_{i}$ é da forma $f\left(t_{i_{1}}, \ldots, t_{i_{m}}\right)$, é claro que queremos dizer que $t_{i}$ é igual a $f\left(t_{i_{1}}, \ldots, t_{i_{m}}\right)$, para uma certo símbolo $f$ e termos $t_{i_{1}}, \ldots, t_{i_{m}}$. Não escrevemos deste modo simplesmente porque queremos distinguir entre a identidade na metalinguagem e a identidade em um modelo.
} 
Definição 1.1.5 Um sistema dedutivo $D^{2}$ para uma lógica $L$ é um conjunto de fórmulas, chamadas de axiomas ${ }^{3}$, junto com um conjunto de relações entre fórmulas, chamadas de Regras de Inferência. A seguinte lista é a lista de (esquemas de) axiomas do sistema D1 da Lógica de Primeira Ordem (Clássica). Sejam $\varphi, \psi$ e $\chi$ fórmulas, $x \in$ Var, $t \in$ Term $e$ $\varphi(x \mid t)$ a fórmula obtida pela substituição de nenhuma ou mais ocorrências de $x$ pelo termo $t$ na fórmula $\varphi$ :

1. $\varphi \rightarrow(\psi \rightarrow \varphi)$

2. $(\varphi \rightarrow \psi) \rightarrow((\varphi \rightarrow(\psi \rightarrow \chi)) \rightarrow(\varphi \rightarrow \chi))$

3. $(\neg \varphi \rightarrow \psi) \rightarrow((\neg \varphi \rightarrow \neg \psi) \rightarrow \varphi)$

4. $\forall x \varphi \rightarrow \varphi(x \mid t)$, desde que o número de ocorrências de variáveis ligadas em $\varphi$ não aumente em $\varphi(x \mid t)$.

As seguintes relações são as Regras de Inferência da Lógica de Primeira Ordem. Sejam $\varphi$, $\psi \in$ Form, $x \in$ Var, $t \in$ Term e em 2. suponha que $x$ não ocorre livre em $\varphi$. As fórmulas em cima da barra são chamadas de "premissas", e a fórmula embaixo dela se chama "conclusão".

$$
\text { 1. Modus Ponens: } \frac{\varphi, \varphi \rightarrow \psi}{\psi} \text {, 2. Generalizaçãa }: \frac{\varphi \rightarrow \psi}{\varphi \rightarrow \forall x \psi} \text {. }
$$

Observação 1.1.6 Aqui introduziremos um abuso de notação. Às vezes escreveremos $\varphi(s)$ para enfatizar que símbolos da sequência s podem estar ocorrendo na fórmula $\varphi$ e, nesse contexto, quando houver uma nova menção de $\varphi$ mas na forma $\varphi\left(s^{\prime}\right)$, estaremos querendo dizer que fizemos a substituição dos símbolos em $s$ que ocorrem em $\varphi$ pelos símbolos em $s^{\prime}$ (obtendo assim uma nova fórmula, digamos $\varphi^{\prime}$ ). Assim, por exemplo, o axioma 4. do sistema $D 1$ poderia ser enunciado como $\forall x \varphi(x) \rightarrow \varphi(t)$.

Definição 1.1.7 Uma prova de $\varphi$ (em um sistema dedutivo D) a partir de um conjunto $\Gamma$ de fórmulas é uma sequencia finita $\varphi_{1}, \ldots, \varphi_{n}$ de fórmulas tal que, para cada $i, 1 \leq i \leq n$,

(a) $\varphi_{i}$ é axioma ou

(b) $\varphi_{i} \in \Gamma$ ou

(c) $\varphi_{i}$ é conclusão de uma ou mais premissas $\varphi_{j}$ através de alguma regra de inferência, com $j<i$.

Neste caso, $\Gamma$ recebe o nome de conjunto de hipóteses, e escrevemos $\Gamma \vdash \varphi$ para dizer que existe uma prova de $\varphi$ a partir de $\Gamma$. Se $\Gamma$ for vazio, dizemos que $\varphi$ é um teorema e escrevemos simplesmente $\vdash \varphi$. Se $\Gamma \vdash \varphi \wedge \neg \varphi$ para alguma $\varphi$, então $\Gamma$ é inconsistente, e caso contrário é consistente. Quando for claro qual sistema dedutivo estiver sendo considerado, não escreveremos o subscrito de $\vdash$.

Semântica. Com frequência encontram-se na literatura diferentes notações e modos de se definir satisfação de primeira ordem. Usaremos aqui uma notação que acreditamos ser a mais clara para nossos propósitos.

No restante desta seção, permanecerá fixada uma linguagem de primeira ordem no vocabulário $V$.

\footnotetext{
${ }^{2}$ Todos os sistemas dedutivos considerados neste trabalho serão recursivos. Isto quer dizer que o sistema $D$ tem duas propriedades: (a) seu conjunto de axiomas é recursivo, isto é, existe uma função recursiva $f$ definida nos números de Gödel das fórmulas tal que se $\# \varphi$ é o número de Gödel de $\varphi$, então $f(\# \varphi)=1$ se $\varphi$ é um axioma de $D$ e $f(\# \varphi)=0$, caso contrário. (b) Suas regras de inferência são recursivas, isto é, para cada regra de inferência $R_{i}$ de $D$ existe uma função recursiva $r_{i}$ tal que se $\psi$ segue de $\varphi_{1}, \ldots, \varphi_{n}$ pelo uso de $R_{i}$, então $r_{i}\left(\# \varphi_{1}, \ldots, \# \varphi_{n}, \# \psi\right)=1$ e, se não, então $r_{i}\left(\# \varphi_{1}, \ldots, \# \varphi_{n}, \# \psi\right)=0$.

${ }^{3}$ Às vezes, por serem axiomas que não são particulares a nenhuma teoria, tais axiomas são chamados de axiomas lógicos.
} 
Definição 1.1.8 Dada uma $V$-estrutura $\langle M, I\rangle$, uma atribuição de valores às variáveis é uma função $s:$ Var $\rightarrow M$; é comum chamarmos tais funções simplesmente de atribuições. Dada uma atribuição $s$, definimos a interpretação $t^{M}[s]$ de $t \in$ Term como sendo um elemento de $M$, segundo as cláusulas:

(a) se $t$ é um simbolo de constante $c$, então $t^{M}[s]=c^{M}$, isto é, $t^{M}[s]=I(c)$.

(b) se té uma variável, então $t^{M}[s]=s(t)$.

(c) se $t$ é da forma $f\left(t_{1}, \ldots, t_{n}\right)$, onde $t_{1}, \ldots, t_{n}$ são termos e $f$ é um símbolo de função $n$ ária, então $t^{M}[s]=f^{M}\left(t_{1}^{M}[s], \ldots, t_{n}^{M}[s]\right)$. Denotaremos às vezes a sequencia $\left(t_{1}^{M}[s], \ldots, t_{n}^{M}[s]\right)$ como $\left(t_{1}^{M}, \ldots, t_{n}^{M}\right)[s]$; assim, neste caso $t^{M}[s]$ fica $f^{M}\left(t_{1}^{M}, \ldots, t_{n}^{M}\right)[s]$.

A função $t \mapsto t^{M}[s]$ às vezes é chamada de denotação dos termos.

Definição 1.1.9 Dada uma estrutura $M$, uma atribuição s e uma fórmula $\varphi$ de primeira ordem, dizemos que $M$ satisfaz $\varphi$ na atribuição $s$, em símbolos $M \vDash \varphi[s]$, se valer um dos casos:

(a) $\varphi$ é atômica $P\left(t_{1}, \ldots, t_{n}\right)$ e $\left\langle t_{1}^{M}, \ldots, t_{n}^{M}\right\rangle[s] \in P^{M}$.

(b) $\varphi$ é da forma $\neg \psi$ e $M$ não satisfaz $\psi$ na atribuição $s$ (em simbolos, $M \not \models \psi[s]$ ).

(c) $\varphi$ é da forma $\psi \rightarrow \chi$ e vale $M \not \models \psi[s]$ ou $M \vDash \chi[s]$.

(d) $\varphi$ é da forma $\forall x \psi$ e para toda atribuição $s^{\prime}:$ Var $\rightarrow M$ tal que para todo y tal que $y \neq x$ temos $s^{\prime}(y)=s(y)$ e $M \vDash \psi\left[s^{\prime}\right]$.

O seguinte resultado é bem conhecido:

Proposição 1.1.10 Seja $V L(\varphi)=\{x \in \operatorname{Var}: x$ ocorre livre em $\varphi\}$, o conjunto das variáveis livres de $\varphi$. Então a relação $M \vDash \varphi[s]$ só depende de $V L(\varphi)$. Mais precisamente,

$$
s \uparrow V L(\varphi)=s^{\prime} \uparrow V L(\varphi) \text { implica } M \vDash \varphi[s] \Leftrightarrow M \vDash \varphi\left[s^{\prime}\right] .
$$

Assim, se $V L(\varphi)=\left\{x_{1}, \ldots, x_{n}\right\}$, também escrevemos $M \vDash \varphi\left[x_{1}\left|a_{1}, \ldots, x_{n}\right| a_{n}\right]$, ou ainda $M \vDash \varphi\left[a_{1}, \ldots, a_{n}\right]$ (se não houver perigo de confusão), para dizer que a estrutura $M$ satisfaz $\varphi$ numa atribuição $s$ qualquer tal que $s\left(x_{1}\right)=a_{1}, \ldots, s\left(x_{n}\right)=a_{n}$. Se $V L(\varphi)=\emptyset$, então a satisfação de $\varphi$ em $M$ não depende de $s$ e escrevemos simplesmente $M \vDash \varphi$. Semelhantemente, se $t$ é um termo sem ocorrência de variáveis, então $t^{M}[s]=t^{M}\left[s^{\prime}\right]$ para quaisquer atribuções $s$ e $s^{\prime}$, e escrevemos então $t^{M}$ para qualquer denotação de $t$.

Definição 1.1.11 A Lógica de Primeira Ordem é o par $\langle L 1, \models\rangle$ tal que L1(V) é o conjunto das sentenças de primeira ordem de vocabulário $V$ dado pela definição 1.1 .4 e $\vDash$ é dada pela definição 1.1.9, juntamente com a proposição 1.1.10. É fácil verificar que $\langle L 1, \models\rangle$ satisfaz as cinco cláusulas da definição 1.1.2.

Definição 1.1.12 (a) Uma fórmula $\varphi$ é universalmente válida, ou simplesmente válida, se $M \vDash \varphi[s]$ para toda estrutura $M$ e toda atribuição s. $\varphi$ é satisfazível, ou tem modelo, se existe uma estrutura $M$ e uma atribuição s tal que $M \vDash \varphi[s]$; neste caso o par $M, s$ é dito um modelo de $\varphi$. Um conjunto $\Gamma$ de fórmulas é satisfazível, ou tem modelo, se existe uma estrutura $M$ e uma atribuição s tais que para toda $\varphi \in \Gamma$ vale $M \vDash \varphi[s]$; neste caso $M \vDash \Gamma[s]$ e dizemos que o par $M, s$ é um modelo para $\Gamma{ }^{4}$

(b) Uma fórmula $\varphi$ é uma consequencia semântica de $\Gamma$ se para todo modelo $M, s$ tal que $M \vDash \Gamma[s]$ temos $M \vDash \varphi[s]$; escrevemos $\Gamma \vDash \varphi$ (apesar de $\vDash$ estar sendo usado para duas noções distintas, a de "satisfação" e a de "consequencia semântica", no contexto deve ficar claro qual o seu significado).

\footnotetext{
${ }^{4}$ Frequentemente quando dissermos que $M$ é um modelo para Gamma, vamos subentender que $V_{M}$ tem apenas os símbolos que ocorrem em $\Gamma$.
} 
A definição de consequencia semântica pode ser generalizada:

Definição 1.1.13 Seja L uma Lógica qualquer e $\Sigma \cup\{\varphi\}$ um conjunto de sentenças de L. Definimos $\Sigma \vDash_{L} \varphi$, ou $\varphi$ é consequencia semântica de $\Sigma$ em relação à $L$, se para toda estrutura $M$ tal que $M \vDash \sigma$ para toda $\sigma \in \Sigma$ temos $M \vDash \varphi$.

Propriedades importantes. Vamos definir agora algumas propriedades muito importantes para uma Lógica arbitrária. Como vamos ver, a semântica padrão da Lógica de Segunda Ordem não satisfará a maioria destas propriedades, contrastando fortemente com a Lógica de Primeira Ordem, que satisfaz a todas. A prova de que $L 1$ satisfaz estas propriedades não será dada aqui; este fato será apenas enunciado na forma de um teorema. Ela pode ser encontrada, por exemplo, em Enderton (2000) e Chang e Keisler (1992). A maior parte das definições que seguem não provém de referências, sendo motivadas por certas definições em Barwise e Feferman (1985). A partir daqui, suponha dada uma Lógica $L$.

Definição 1.1.14 Propriedade da Completude. Existe um sistema dedutivo $D$ tal que para toda $\varphi$, se $\Gamma \vDash_{L} \varphi$, então $\Gamma \vdash_{D} \varphi$.

Definição 1.1.15 Propriedade da Compacidade. Se todo subconjunto finito de $\Gamma$ tem modelo, então $\Gamma$ tem modelo.

Para as definições de Löwenheim-Skolem vamos precisar da noção de subestrutura:

Definição 1.1.16 Sejam $\langle M, I\rangle$ e $\left\langle M^{\prime}, I^{\prime}\right\rangle V$-estruturas. $M^{\prime}$ é subestrutura de $M$ se $M^{\prime} \subseteq$ $M$ e, para cada símbolo $v \in V, I^{\prime}(v)$ é uma "restrição" de $I(v)$ em $M^{\prime}$, isto é,

(i) se $v$ é um símbolo de constante, então $I^{\prime}(v)=I(v)$,

(ii) se vé um símbolo de função n-ária, então $I^{\prime}(v)=I(v) \uparrow\left(M^{\prime}\right)^{n} e$

(iii) se $v$ é um símbolo de relação n-ária, então $I^{\prime}(v)=I(v) \cap\left(M^{\prime}\right)^{n}$.

Definição 1.1.17 Propriedade de Löwenheim-Skolem para baixo. Se $M$ é uma estrutura, então $M$ tem uma subestrutura $M^{\prime}$ com as seguinte propriedades: a cardinalidade de $M^{\prime}$ é no máximo a cardinalidade do vocabulário de $M$ ou infinita enumerável, a que for maior, e se s é uma atribuição que só assume valores em $M^{\prime}$, então

$$
M^{\prime} \vDash_{L} \varphi[s] \Leftrightarrow M \vDash_{L} \varphi[s] .
$$

Em particular, se $\Gamma$ tem modelo, então $\Gamma$ tem um modelo com dominio de cardinalidade no máximo a do conjunto dos símbolos usados em $\Gamma$ ou infinita enumerável, a que for maior.

Definição 1.1.18 Propriedade de Löwenheim-Skolem para cima. Se $\Gamma$ tem modelo de cardinalidade menor ou igual a $\lambda$, e se o vocabulário de $\Gamma$ também tiver cardinalidade menor ou igual a $\lambda$, então $\Gamma$ tem modelo de cardinalidade $\lambda$.

A próxima definição exige que a Lógica $L$ em questão possua a noção de variáveis livres.

Definição 1.1.19 Propriedade da Omissão de tipos. Suponha que T seja um conjunto de sentenças satisfazivel e que $\Gamma$ seja um conjunto de fórmulas maximal satisfazível em $\left\{\Sigma \subseteq\right.$ Form $: T \subseteq \Sigma$ e $\left.\varphi \in \Sigma \Rightarrow V L(\varphi) \subseteq\left\{x_{1}, \ldots, x_{n}\right\}\right\}$ (tal $\Gamma$ é chamado de um n-tipo sobre T). Suponha também que não existe $\varphi$ tal que para toda $\psi \in \Gamma$ temos $T \vDash_{L} \varphi \rightarrow \psi$ (ou seja, $\Gamma$ não é isolado) e que o vocabulário em questão seja enumerável. Então existe uma estrutura $M$ enumerável tal que $M \vDash T$ e existem $a_{1}, \ldots, a_{n}$ tais que $M \not \nvdash_{L} \Gamma\left[a_{1}, \ldots, a_{n}\right]$. 


\subsection{Sintaxe e Semântica Padrão de L2}

Vamos começar a definir agora o que seria Lógica de Segunda Ordem. O leitor notará que, diferentemente do que fizemos para Primeira Ordem, descreveremos as estruturas antes de definirmos o sistema dedutivo que usaremos. O porque disto ficará mais claro adiante.

Há mais de um modo de se estender uma linguagem de primeira ordem para que ela possa se referir à totalidade das relações entre os objetos. A mais direta e despreocupada é simplesmente adicionar variáveis de relação $n$-árias e permitir quantificação sobre estas variáveis, e esta é a posição adotada pela semântica padrão. Outras formas podem ser encontradas na literatura, tais como permitir apenas variáveis de relações unárias (Lógica de Segunda Ordem Monádica), introduzir as variáveis mas não permitir quantificação sobre elas (Lógica de Segunda Ordem Livre), ou ainda permitir apenas a formação de relações que possam ser definidas em termos de relações já definidas (Lógica de Segunda Ordem Predicativa). Cada uma destas formas tem seus méritos, que não serão discutidos aqui. O leitor interessado pode consultar Shapiro (2000).

Definição 1.2.1 A linguagem de segunda ordem com vocabulário $V$ é a tripla $\langle$ Alf, Term, Form $\rangle$, onde

(a) $A l f=A l f_{1} \cup \operatorname{Var}_{2}^{F} \cup \operatorname{Var}_{2}^{R}$ é uma união disjunta e Alf $f_{1}$ é um alfabeto de uma linguagem de primeira ordem com vocabulário $V e$

(i) $\operatorname{Var}_{2}^{F}=\bigcup_{n>1} \operatorname{Var}_{2}(F, n)$ é uma união disjunta, chamada de conjunto das variáveis (de segunda ordem) de funções. Se $f \in \operatorname{Var}_{2}(F, n)$, então $f$ é dita uma variável de função $n$ ária.

(ii) $\operatorname{Var}_{2}^{R}=\bigcup_{n \geq 1} \operatorname{Var}_{2}(R, n)$ é uma união disjunta, chamada de conjunto das variáveis (de segunda ordem) de relações. Se $X \in \operatorname{Var}_{2}(R, n)$, então $X$ é dita uma variável de relação $n$-ária.

Usaremos a notação Var ${ }_{1}$ para as variáveis em Alf $f_{1}$. Escreveremos $\operatorname{Var}=\operatorname{Var}_{1} \cup \operatorname{Var}_{2}^{F} \cup$ $\operatorname{Var}_{2}^{R}$ para o conjunto das variáveis.

(b) Term é um conjunto tal que $t \in$ Term se, e somente se, existe uma sequencia $t_{1}, \ldots, t_{n}$ tal que $t_{n}$ ét e, para cada $i, 1 \leq i \leq n$,

(i) $t_{i} \in \operatorname{Var}_{1}$ ou

(ii) $t_{i}$ é símbolo de constante ou

(iii) $t_{i}$ é da forma $f\left(t_{i_{1}}, t_{i_{2}}, \ldots, t_{i_{m}}\right)$, onde $f \in F_{m} \cup \operatorname{Var}_{2}(F, m)$ e $i_{1}, i_{2}, \ldots, i_{m}<i$.

$A$ complexidade $c(t)$ de um termo $t$ é definida como em 1.1.4.

(c) Form é um conjunto tal que $\varphi \in$ Form se, e somente se, existe uma sequencia $\varphi_{1}, \ldots, \varphi_{n}$ tal que $\varphi_{n}$ é $\varphi$ e, para cada $i, 1 \leq i \leq n$,

(i) $\varphi_{i}$ é $P\left(t_{1}, \ldots, t_{m}\right)$, com $P \in R_{m} \cup \operatorname{Var}_{2}(R, m)$ e $t_{1}, \ldots, t_{m} \in$ Term, caso em que $\varphi$ é dita atômica, ou

(ii) $\varphi_{i}$ é $\neg \varphi_{j}$ ou $\varphi_{i}$ é $\varphi_{j} \rightarrow \varphi_{k}$, com $j, k<i$, ou

iii) (Quantificação de primeira ordem) $\varphi_{i}$ é $\forall x \varphi_{j}$, com $x \in \operatorname{Var}_{1}$ e $j<i$, ou

(iv) (Quantificação de funções) $\varphi_{i}$ é $\forall f \varphi_{j}$, com $f \in \operatorname{Var}_{2}^{F}$ e $j<i$.

(v) (Quantificação de relações) $\varphi_{i}$ é $\forall X \varphi_{j}$, com $X \in \operatorname{Var}_{2}^{R}$ e $j<i$.

A complexidade $c(\varphi)$ de uma fórmula $\varphi$, a ocorrência de variáveis livres e ligadas, a definição de uma sentença e a definição dos conectivos usuais restantes são feitos análogos a 1.1.4.

A Semântica Padrão. Queremos agora definir uma relação de satisfação para $L 2$. Há uma maneira muito natural de fazê-lo para uma linguagem de segunda ordem, que é simplesmente interpretar quantificadores de segunda ordem $\forall X$, com $X n$-ária, como tomando valores sobre a coleção de todos os subconjuntos $X$ do produto cartesiano $n$-ário do domínio; 
esta é a semântica chamada padrão. Essa não é a única maneira, contudo; iremos ver outra muito importante logo adiante, chamada de semântica de Henkin. A semântica padrão, como iremos mostrar, não satisfaz nenhuma das propriedades 1.1.14 a 1.1.19.

Definição 1.2.2 Dado uma estrutura $M$, definimos uma atribuição s para variáveis de primeira e segunda ordem como uma função $s: \operatorname{Var}_{1} \cup \operatorname{Var}_{2}^{F} \cup \operatorname{Var}_{2}^{R} \rightarrow M \cup \bigcup_{n \geq 1} P\left(M^{n}\right)$ tal que

(a) se $x \in \operatorname{Var}_{1}$, então $s(x) \in M$,

(b) se $f \in \operatorname{Var}_{2}(F, n)$, então $s(f) \in P\left(M^{n+1}\right)$ e é função, com $s(f): M^{n} \rightarrow M e$

(c) se $X \in \operatorname{Var}_{2}(R, n)$, então $s(X) \in P\left(M^{n}\right)$.

Dada a atribuição s, definimos a interpretação $t^{M}[s]$ de $t \in$ Term como em 1.1.8, adicionando a cláusula de que se $t$ é $f\left(t_{1}, \ldots, t_{n}\right)$ com $f \in \operatorname{Var}_{2}(F, n)$, então $t^{M}[s]=s(f)\left(t_{1}^{M}, \ldots, t_{n}^{M}\right)[s]$.

Definição 1.2.3 Dado um modelo $M$ e uma atribuição s para variáveis de primeira e segunda ordem e uma fórmula $\varphi$, dizemos que $M$ satisfaz $\varphi$ na atribuição $s$ se

(a) $\varphi$ for $X\left(t_{1}, t_{2}, \ldots, t_{n}\right)$ com $X \in R_{n}$ e for o caso de $\left\langle t_{1}^{M}, t_{2}^{M}, \ldots, t_{n}^{M}\right\rangle[s] \in X^{M}$; se $X \in \operatorname{Var}_{2}(R, n)$, tem de ser o caso de $\left(t_{1}^{M}, t_{2}^{M}, \ldots, t_{n}^{M}\right) \in s(X)$.

Os casos (b), (c) e (d) nos quais $\varphi$ é da forma $\neg \psi, \psi \rightarrow \chi e \forall x \psi$, com $x \in \operatorname{Var}_{1}$, são exatamente como em primeira ordem, definição 1.1.4. A nova cláusula é como esperada:

(e) $\varphi$ é da forma $\forall X \psi$ com $X \in \operatorname{Var}_{2}^{R} \cup \operatorname{Var}_{2}^{F}$ e, se para toda atribuição $s^{\prime}$ tal que $Y \neq X$ e $s^{\prime}(Y)=s(Y)$, temos $M \vDash \psi\left[s^{\prime}\right]$.

A definição 1.1.12 também faz sentido em relação à semântica padrão de L2, com as alterações óbvias, e vamos usá-la. Análogamente, a Proposição 1.1.10, que diz que a satisfação só depende de $V L(\varphi)$, também é válida para fórmulas de segunda ordem.

Definição 1.2.4 A Lógica de Segunda Ordem (com a semântica padrão) é o par $\langle L 2, \vDash\rangle$ tal que $L 2(V)$ é o conjunto das sentenças de segunda ordem de vocabulário $V$ dado pela definição 1.2.1 e $\vDash$ é dado pela definição 1.2.3.

Passaremos agora a mostrar que as propriedades de 1.1 .14 a 1.1 .19 não são satisfeitas pela semântica padrão. Compacidade pode ser refutada de imediato:

Proposição 1.2.5 A semântica padrão não satisfaz a propriedade da Compacidade (1.1.15).

Prova. Considere o seguinte conjunto de sentenças:

$$
\begin{gathered}
\forall f[\forall x \forall y(f x=f y \rightarrow x=y) \rightarrow \forall y \exists x(f x=y)], \\
\exists x_{1} \exists x_{2}\left(x_{1} \neq x_{2}\right), \exists x_{1} \exists x_{2} \exists x_{3}\left(x_{1} \neq x_{2} \wedge x_{1} \neq x_{3} \wedge x_{2} \neq x_{3}\right), \ldots
\end{gathered}
$$

Isto é, a primeira sentença diz que toda função injetora é sobrejetora, e as outras são uma coleção infinita de sentenças cada uma dizendo que existem ao menos $n$ elementos, com $n \geq 2$. Então cada subconjunto finito destas sentenças tem um modelo (um conjunto finito), mas não existe um modelo de todas elas.

Sabemos que em Lógica de Primeira Ordem, com o auxílio do sistema dedutivo D1 (ou algum outro que prova as mesmas fórmulas que $D 1$ ), que as propriedades da Compacidade e da Completude são equivalentes (Enderton (2000)). No entanto, pela forma como estamos enunciando estas propriedades, elas não precisam ser equivalentes numa lógica arbitrária e, assim, não segue que Completude não é válida em $L 2$ apenas a partir da última proposição. Para ver que não vale a Completude em $L 2$, vamos mostrar que a Semântica Padrão não é axiomatizável, isto é, não existe um sistema dedutivo para as fórmulas universalmente 
válidas de $L 2{ }^{5}$; foi por conta disto que escolhemos discutir a semântica antes de escolher um sistema dedutivo. Para este resultado, vamos precisar mostrar que a teoria da aritmética de segunda ordem é categórica, isto é, que ela tem um único modelo a menos de isomorfismo. Isso também vai garantir, é claro, que $L 2$ não satisfaz a propriedade de Löwenheim-Skolem para cima (1.1.18).

Na verdade, a situação é ainda mais complexa: o conjunto das fórmulas de segunda ordem universalmente válidas não é nem ao menos definível na hierarquia analítica e nem por nenhuma fórmula de primeira ordem nos modelos $\left\langle V_{\kappa}, \in\right\rangle$ da Teoria de Conjuntos, com $\kappa$ o primeiro cardinal inacessível. Entretanto, estes últimos resultados vamos deixar mais para frente, para quando estivermos discutindo a metateoria da Semântica Padrão de modo mais aprofundado.

Teorema 1.2.6 Categoricidade da Aritmética. Se $M_{1}$ e $M_{2}$ são modelos (padrão) dos axiomas da função sucessor,

$$
\forall x(s x \neq 0), \forall x \forall y(s x=s y \rightarrow x=y)
$$

e do princípio de indução de segunda ordem,

$$
\forall X[(X 0 \wedge \forall x(X x \rightarrow X s x)) \rightarrow \forall x X x]
$$

(estes axiomas mais as definições recursivas da soma ${ }^{6}$ e do produto ${ }^{7}$ são chamados de axiomas de segunda ordem da aritmética de Peano), então $M_{1} \uparrow\{0, s\} \cong M_{2} \uparrow\{0, s\}$.

Prova. Diga que $S \subseteq M_{1} \times M_{2}$ é fechado por sucessor se $\left\langle 0^{M_{1}}, 0^{M_{2}}\right\rangle \in S$ e se $\langle a, b\rangle \in S$ então $\left\langle s^{M_{1}} a, s^{M_{2}} b\right\rangle \in S$, e considere $f=\bigcap\{S: S$ é fechado por sucessor $\}$. Como $M_{1} \times M_{2}$ é fechado por sucessor, existe $f$ e $f \neq \emptyset$, já que $\left\langle 0^{M_{1}}, 0^{M_{2}}\right\rangle \in f$. Além disso, $f$ é fechado por sucessor. Vamos mostrar que $f$ é o isomorfismo procurado.

Primeiro vamos provar que $\operatorname{dom}(f)=M_{1}$. Considere $D=\left\{a \in M_{1}: \exists b(\langle a, b\rangle \in f)\right\}$, o domínio de $f$. Como $\left\langle 0^{M_{1}}, 0^{M_{2}}\right\rangle \in f$, temos $0^{M_{1}} \in D$. Suponha que $\langle a, b\rangle \in f$; como $f$ é fechado por sucessor, $\left\langle s^{M_{1}} a, s^{M_{2}} b\right\rangle \in f$. Como $M_{1}$ satisfaz o axioma de indução de segunda ordem, $D=M_{1}$ (observe aqui o uso da relação de satisfação da semântica padrão). Para ver que $f$ é uma função bijetora, considere agora $P=\left\{a \in M_{1}: \exists ! b(\langle a, b\rangle \in f)\right\}$. Se existisse $\left\langle 0^{M_{1}}, c\right\rangle \in f$ tal que $c \neq 0^{M_{2}}$, então $f-\left\{\left\langle 0^{M_{1}}, c\right\rangle\right\}$ seria fechado por sucessor, e pela definição de $f$ temos $f \subseteq f-\left\{\left\langle 0^{M_{1}}, c\right\rangle\right\}$, um absurdo. O passo de indução é semelhante: dado $\langle a, b\rangle \in f$, se $\left\langle s^{M_{1}} a, c\right\rangle \in f$ e $c \neq s^{M_{2}} b$, então $f-\left\{\left\langle s^{M_{1}} a, c\right\rangle\right\}$ é fechado por sucessor, e então $f \subseteq f-\left\{\left\langle s^{M_{1}} a, c\right\rangle\right\}$. Logo $f$ é uma função. Além disso, o mesmo argumento usando o princípio de indução em $M_{2}$ mostra que $\operatorname{dom}\left(f^{-1}\right)=M_{2}$ e que $f^{-1}$ é função. Logo $f$ é bijetora. Para ver que $f$ é um isomorfismo, basta notar que pelo fato de ser fechado por sucessor, temos $f\left(0^{M_{1}}\right)=0^{M_{2}}$ e $f\left(s^{M_{1}} a\right)=s^{M_{2}}(f a)$, para todo $a \in M_{1}$.

Corolário 1.2.7 Löwenheim-Skolem para cima (1.1.18) não é satisfeito pela semântica padrão.

Observação 1.2.8 Fórmulas com símbolos não-lógicos podem ser trocadas por fórmulas que usam apenas símbolos lógicos (e linguisticos) em L2 e continuar tendo essencialmente os mesmos modelos. Vamos ilustrar o método com um exemplo: todo modelo de

\footnotetext{
${ }^{5}$ Isto às vezes é chamado de completude fraca.

${ }^{6} \forall x(x+0=x) \wedge \forall x \forall y(x+s y=s(x+y))$

${ }^{7} \forall x(x .0=0) \wedge \forall x \forall y(x . s y=x . y+x)$
} 


$$
\forall x(s x \neq 0) \wedge \forall x \forall y(s x=s y \rightarrow x=y)
$$

também é modelo de

$$
\exists f[\exists w \forall x(f x \neq w) \wedge \forall x \forall y(f x=f y \rightarrow x=y)],
$$

e é claro que todo modelo desta última fórmula pode ser estendido a um modelo da primeira (a não ser, é claro, que este modelo já tenha uma interpretação para os símbolos s ou 0).

Corolário 1.2.9 (a) O conjunto das fórmulas universalmente válidas da Lógica de Segunda Ordem não é definivel em $N$, o modelo dos números naturais, por nenhuma fórmula de segunda ordem.

(b) L2 com a semântica padrão não é axiomatizável (e assim não temos Completude).

Prova. (a) Sejam $T_{2}(N)$ o conjunto das sentenças da aritmética de segunda ordem válidas no modelo dos naturais, $P 2$ a conjunção dos axiomas de segunda ordem da aritmética de Peano e $\varphi$ uma sentença qualquer de $L 2(s, 0)$. Então

$$
\varphi \in T_{2}(N) \Leftrightarrow \vDash P 2 \rightarrow \varphi,
$$

já que $P 2$ só admite um modelo a menos de isomorfismo. Agora, o lado esquerdo da equivalência é uma condição (sobre os números de Gödel das fórmulas) não definível em $N$ por nenhuma fórmula de segunda ordem, pelo Teorema da Indefinibilidade de Tarski (cuja prova pode ser encontrada em Enderton (2000), e a demonstração para $L 2$ é a mesma que para $L 1)$ e, consequentemente, o lado direito também não é. Pela observação $1.2 .8, P 2 \rightarrow \varphi$ é equivalente (pode ser trocada recursivamente) por uma fórmula da linguagem de segunda ordem pura, isto é, uma fórmula sem símbolos lógicos, cuja interpretação não depende do vocabulário e assim é válida em todos os modelos. Logo, dentro do conjunto (dos números de Gödel) das fórmulas de segunda ordem universalmente válidas, existe um conjunto que não é definível em $N$, e é fácil de ver que se aquele conjunto fosse definível, este também seria.

(b) Pelo item (a), o conjunto das fórmulas universalmente válidas de $L 2$ não é recursivamente enumerável. Como o conjunto dos teoremas de um sistema dedutivo recursivo é recursivamente enumerável, segue a tese.

Vamos mostrar agora que a teoria de segunda ordem da Reta Real também é categórica; como a cardinalidade do conjunto dos números reais é maior que a dos naturais, vai seguir que a propriedade de Löwenheim-Skolem para baixo 1.1.17 também não se verifica na Semântica Padrão.

Teorema 1.2.10 Suponha que $M$ e $M^{\prime}$ sejam modelos dos axiomas de corpo ordenado (com vocabulário $\{0,1,+, \cdot, \leq\})$ :

$$
\begin{array}{ll}
\forall x \forall y \forall z((x+y)+z=x+(y+z)) & \forall x \forall y(x+y=y+x) \\
\forall x(x+0=x) & \forall x \exists y(x+y=0) \\
\forall x \forall y \forall z((x . y) . z=x .(y . z)) & \forall x \forall y(x . y=y \cdot x) \\
\forall x(x .1=x) & \forall x(x \neq 0 \rightarrow \exists y(x . y=1)) \\
\forall x \forall y \forall z((x .(y+z)=(x . y)+(x . z)) & \\
\forall x \forall y \forall z((x \leq y \wedge y \leq z) \rightarrow x \leq z) & \\
\forall x \forall y((x \leq y \wedge y \leq x) \rightarrow x=y) & \\
\forall x \forall y((0<x \wedge 0<y) \rightarrow(0<x+y \wedge 0<x . y)) &
\end{array}
$$

e que também satisfaçam o axioma de completude de segunda ordem, que diz que todo conjunto não vazio limitado superiormente tem um menor limitante superior, isto é, um supremo: 
$\forall X\{\exists x(X x) \wedge \exists x \forall y(X y \rightarrow y \leq x) \rightarrow \exists x[\forall y(X y \rightarrow y \leq x) \wedge \forall z(\forall y(X y \rightarrow y \leq z) \rightarrow x \leq z)]\}$

Então $M \cong M^{\prime}$.

Prova. É feita em várias etapas; vamos construir o isomorfismo explícitamente. Considere $|N|$ o menor subconjunto de $|M|$ formado pela interpretação do 0 , do 1 e fechado pela soma e seja $N$ a subestrutura de $M \uparrow\{0,1,+, \cdot\}$ de domínio $|N|$. Então, é fácil ver que temos

Lema Seja P2* a fórmula P2 trocando toda ocorrência de $s(-)$ por $(-)+1$. Então todo modelo de $P 2$ pode ser extendido a um modelo de $P 2 *$ e, reciprocamente, se $N$ for um modelo de $P 2 *$, então $N$ pode ser extendido a um modelo de P2.

Assim, se $N^{\prime}$ é o submodelo óbvio de $M^{\prime} \uparrow\{0,1,+,$.$\} , como N$ e $N^{\prime}$ satisfazem $P 2 *$, pelo lema e pelo teorema 1.2.6 existe um isomorfismo $f: N \rightarrow N^{\prime}$ (note que 1.2.6 garante um isomorfismo, entretanto, com modelos de vocabulário $\{s, 0\}$. Mas é fácil de ver que se adicionarmos os axiomas recursivos da soma e do produto, então o teorema continua valendo, e como a soma e o produto de $M$ satisfazem estes axiomas, não há qualquer problema). O que vamos fazer é estender essa função a um isomorfismo entre $M$ e $M^{\prime}$. Para isso, considere primeiro o subconjunto $|Z|=|N| \cup\{-a: a \in|N|\}$ de $|M|^{8}$ e seja $Z$ o submodelo óbvio de $M$. Então, se $Z^{\prime}$ é o submodelo correspondente de $M^{\prime}, f$ pode ser estendida a um isomorfismo de $Z$ em $Z^{\prime}$ pondo $f(-a)=-f(a)$ para todo $a \in|Z|-|N|$ (para ver que $f$ vai preservar a ordem, prove por indução que $0 \leq n \Rightarrow 0 \leq f(n)$, para todo $n \in|N|$. Daí $a \leq b \Leftrightarrow 0 \leq b-a \Rightarrow 0 \leq f(b)-f(a) \Rightarrow f(a) \leq f(b)$. Como $f$ é bijetora, vai ter que valer também $f(a) \leq f(b) \Rightarrow a \leq b)$. A partir daí, defina o subconjunto $|Q|$ de $|M|$ construído indutivamente da seguinte forma:

$$
\begin{aligned}
|Q|_{0} & =|Z| \\
|Q|_{n+1} & =|Q|_{n} \cup\left\{a . b, a^{-1}: a, b \in|Q|_{n}\right\}, \\
|Q| & =\bigcup_{n}|Q|_{n} .
\end{aligned}
$$

Por indução em $n$ nos $|Q|_{n}$, pode-se mostrar que todo elemento de $|Q|$ é da forma $p . q^{-1}$, $\operatorname{com} p, q \in|Z|$; estenda então $f$ a $|Q|$ assim: $f\left(p \cdot q^{-1}\right)=f(p) \cdot f(q)^{-1}$. Então $f$ pode fácilmente ser vista bijetora sobre o subconjunto $\left|Q^{\prime}\right|$ correspondente de $\left|M^{\prime}\right|$ (faça uma indução nos $\left.\left|Q^{\prime}\right|_{n}\right)$. Além disso, como $p \cdot q^{-1}+r . s^{-1}=(p . s+r . q) \cdot(q s)^{-1},|Q|$ é fechado pela soma, e assim faz sentido falar na subestrutura $Q$ de $M$. Finalmente, da definição de $f$ em $|Q|$ e do fato de $f: Z \cong Z^{\prime}$, temos $f: Q \cong Q^{\prime}$.

Vamos, agora, estender $f$ a $M$. Seja então $a \notin|Q|$ e considere

$$
\overleftarrow{a}=\{q \in|M|: q \in|Q| \text { e } q<a\}
$$

Então $a=\sup \overleftarrow{a}$, o supremo de $\overleftarrow{a}$; de fato, $a$ é um limitante superior de $\overleftarrow{a}$. Para ver que é o menor, usamos o fato que $Q$ é denso ${ }^{9}$ em $M$ o que, por sua vez, depende da prova de que $M$ satisfaz a propriedade arquimediana, uma propriedade que depende do axioma de segunda ordem da completude ${ }^{10}$ :

\footnotetext{
${ }^{8}$ Mais precisamente, estamos tomando os $-{ }^{M} a$. Vamos nos abster de escrever vários sobrescritos deste tipo. O contexto deverá deixar claro o que está sendo feito.

${ }^{9}(|P|, \leq)$ é denso em $\left(\left|P^{\prime}\right|, \leq^{\prime}\right)$ se $P$ é subestrutura de $P^{\prime}$ e se para todos $x, y \in\left|P^{\prime}\right|$ com $x<y$ existe $z \in|P|$ tal que que $x<z<y$.

${ }^{10}$ Depende porque existem corpos ordenados não arquimedianos; uma construção de um corpo assim pode ser encontrada para L1 em Enderton (2000). Observe contudo que esta propriedade não depende da semântica padrão: de fato, para fazer a prova, só precisamos que exista "o menor conjunto que contém a denotação do 0 e do 1 e é fechado pela soma", que é definível em segunda ordem pela fórmula $N(x) \leftrightarrow$
} 
Lema. Se $x \in|M|$, então existe $n \in|N|$ tal que $x<n$.

Prova. Suponha por absurdo que $n \leq x$ para todo $n \in|N|$. Por completude, existe $u=$ $\sup |N|$; mas então $u-1$ não é limitante superior de $|N|$ (já que é menor que $u$ ) e assim existe $m \in|N|$ tal que $u-1<m$, donde $u<m+1$, contradizendo a definição de $u$.

Como um corolário, temos que dados $a, b \in|M|$, com $a<b$, existe $m \in|N|$ tal que $1<m \cdot(b-a)$. Assim, m.a $+1<m \cdot b$. Considere agora

$$
\begin{gathered}
n=\max \{n \in|N|: n \leq m . a\} . \text { Então } \\
m . a<n+1 \leq m \cdot a+1<m . b, \text { e assim } \\
a<m^{-1} \cdot(n+1)<b,
\end{gathered}
$$

donde $Q$ é denso em $M$. Consequentemente, se $b<a$ é tal que para todo $q \in \overleftarrow{a}$ temos $q \leq b$, então existe $q_{0} \in|Q|$ tal que $b<q_{0}<a$. Mas então $q_{0} \in \overleftarrow{a}$, o que implica $q_{0} \leq b$, um absurdo.

Seja agora $n \in|N|$ tal que $a \leq n$; então, pelo isomorfismo em $Q$, para todo $q \in \overleftarrow{a}$ temos $f(q) \leq f(n)$, e portanto $f_{*} \overleftarrow{a}=\{f(q): q \in \overleftarrow{a}\}$ tem supremo. Como o supremo de um conjunto é único, podemos estender $f$ à função $f \cup\left\{\left\langle a, s u p f_{*} \overleftarrow{a}\right\rangle\right\}$. Faça isto para cada $a \notin|Q|$.

Para ver que $f$ é sobrejetora, seja $a^{\prime} \in\left|M^{\prime}\right|$ e considere $\left\{q \in|Q|: f(q)<a^{\prime}\right\}$; este subconjunto de $|M|$ tem um supremo $a$ : de fato, seja $p^{\prime} \in\left|Q^{\prime}\right|$ tal que $a^{\prime}<p^{\prime}$ e $p$ com $f(p)=p^{\prime}$. Se $\left\{q \in|Q|: f(q)<a^{\prime}\right\}$ é ilimitado, tome $q$ neste conjunto com $p<q$. Mas então $a^{\prime}<p^{\prime}=f(p) \leq f(q)<a^{\prime}$, um absurdo. Considere agora $f^{*} \overleftarrow{a^{\prime}}=\left\{q \in|Q|: f(q) \in \overleftarrow{a^{\prime}}\right\}$ vamos mostrar que

$$
f^{*} \overleftarrow{a^{\prime}}=\overleftarrow{a}
$$

Ora, se $q \in f^{*} \overleftarrow{a^{\prime}}$, então $q=f^{-1}\left(q^{\prime}\right)$ para algum $q^{\prime}<a^{\prime}$. Logo $f(q)<a^{\prime}$, e assim por definição de $a$ temos $q<a$. Se $q<a$, por definição de $a$ e pela densidade de $Q$, existe $q_{0} \in|Q|$ tal que $q<q_{0}<a$ e $f\left(q_{0}\right)<a^{\prime}$. Como $q<q_{0} \Rightarrow f(q)<f\left(q_{0}\right)$, temos $f(q)<a^{\prime}$, donde $q \in f^{*} \overleftarrow{a^{\prime}}$.

Assim, $f(a)=\sup _{*}\{q \in|Q|: q<a\}=\sup _{*} f^{*} \overleftarrow{a^{\prime}}=\sup \overleftarrow{a^{\prime}}=a^{\prime}$ (a penúltima igualdade segue por que $f$ é bijetora em $|Q|)$. Provamos então que $f$ é sobrejetora. Para ver que é injetora, suponha que $f(a)=f(b)$ e que $a<b$. Então existe $q \in|Q|$ tal que $a<q<b$. Das considerações anteriores segue que precisamos ter $f(q)<f(b)$. Agora, se fosse o caso de $f(q) \leq f(a)$, como $f: Q \cong Q^{\prime}$, teríamos $f(q)<f(a)$, donde $q<a$. Falta verificar que $f$ preserva a ordem, a soma e o produto.

Para ver que preserva a ordem, suponha $a<b$ e $f(b)<f(a)$ (já sabemos que $f(a) \neq f(b)$ pois $f$ é bijetora). Seja então $p^{\prime} \in\left|Q^{\prime}\right| \operatorname{com} f(b)<p^{\prime}<f(a)$, e $p \operatorname{com} f(p)=p^{\prime}$. Então, de modo análogo ao que foi feito acima, concluímos que $b<p<a$, um absurdo. Para ver que preserva produto, mostre primeiro que $f(p . b)=f(p) . f(b)$ para todo $p \in|Q|$ e todo $b \in|M|$, e depois que $f(a . b)=f(a) . f(b)$ para todos $a, b \in|M|$. A soma se prova análogamente.

Corolário 1.2.11 A propriedade de Löwenheim-Skolem para baixo (1.1.17) não se verifica para a Lógica de Segunda Ordem na Semântica Padrão.

Observação 1.2.12 Sobre teorias de primeira ordem e suas "contrapartes" de segunda ordem. Como se sabe, a teoria de primeira ordem da Aritmética de Peano é a teoria dada pelos axiomas da função sucessor, os axiomas recursivos da soma e do produto mais o esquema de axiomas de indução, isto é, para cada fórmula $\varphi$ de $L 1(0, s,+, \cdot)$, é um axioma

$\forall X(\forall y \forall z(X y \wedge X z \rightarrow X(y+z)) \rightarrow X x)$. Agora, que este conjunto é uma cópia isomorfa do conjunto dos números naturais, isto sim segue do fato de estarmos usando a semântica padrão, pois em outra semântica talvez não existisse o conjunto $\left\{0^{M}, S^{M}\left(0^{M}\right), \ldots\right\}$. 


$$
\forall x[\varphi(x \mid 0) \wedge(\varphi \rightarrow \varphi(x \mid s x))] \rightarrow \forall x \varphi .
$$

Análogamente, a teoria dos corpos reais fechados consiste dos axiomas de corpo ordenado mais o esquema de axiomas:

$$
(\exists x \varphi \wedge \exists x \forall y \varphi(y) \rightarrow y \leq x) \rightarrow \exists x[\forall y(\varphi(y) \rightarrow y \leq x) \wedge \forall z(\forall y(\varphi(y) \rightarrow y \leq z) \rightarrow x \leq z)],
$$

onde $\varphi \in L(0,1,+, ., \leq)$. Isto sugere um procedimento para se obter uma teoria de primeira ordem a partir de uma de segunda (e vice-versa), a saber: se todas as fórmulas da teoria são da forma $\forall X_{1} \forall X_{2} \ldots \forall X_{n} \varphi$, onde $X_{1}, X_{2}, \ldots, X_{n}$ são variáveis de segunda ordem e $\varphi$ é uma fórmula onde não há ocorrência de quantificadores de segunda ordem (permitindo-se $n=0$ ), fazemos o seguinte: para cada fórmula $\forall X_{1} \forall X_{2} \ldots \forall X_{n} \varphi$, tomamos $\varphi$ e substituímos as ocorrências de cada $X_{1}, X_{2}, \ldots, X_{n}$ por fórmulas $\varphi_{1}, \varphi_{2}, \ldots, \varphi_{n}$ de primeira ordem no vocabulário em questão. Mais para frente veremos que, tomando-se a teoria de conjuntos de segunda ordem ZF2 e aplicando-se este procedimento, obtemos a teoria de conjuntos $Z F$. Assim, em alguma medida, podemos dizer que este procedimento é natural. Mas, contudo, ele não define uma única classe de modelos para as contrapartes de primeira ordem, isto é, é possivel achar duas teorias de segunda ordem que possuem a mesma classe de modelos mas que suas contrapartes de primeira ordem (neste sentido) tem classes de modelos diferentes (Montague (1965)).

Por conta de $L 1$ satisfazer a propriedade de Löwenheim-Skolem para cima, não existe um conjunto $\Gamma$ de fórmulas de $L 1$ cujas estruturas sejam todas finitas, isto é, a classe das estruturas finitas não é definível em primeira ordem. Na verdade, se $\Gamma$ é satisfazível, então sempre existe um cardinal suficientemente grande para o qual $\Gamma$ tem modelo desta cardinalidade. A semântica padrão de $L 2$ difere de $L 1$ também neste tema.

Teorema 1.2.13 (i) Existe uma fórmula $\sigma_{<\omega} \in L 2$ que se verifica somente nas estruturas finitas.

(ii) Para cada cardinal $\aleph_{n}$ existe uma fórmula $\sigma_{\aleph_{n}}(X)$ tal que $\sigma_{\aleph_{n}}(X)$ se verifica para o conjunto $X$ (isto é, para a relação unária $X$ ) se e somente se o cardinal de $X$ é $\aleph_{n}$.

Prova. (i) Seja $\operatorname{Inj}(f): \forall x \forall y(f x=f y \rightarrow x=y)$ e $S b j(f): \forall x \exists y(f y=x)$. Então $\sigma_{<\omega}$ pode ser tomada como $\forall f(\operatorname{Inj}(f) \rightarrow \operatorname{Sbj}(f))$.

(ii) A fórmula $\sigma_{<\omega}$ pode ser modificada para dizer que símbolos unários são finitos. Defina Fun $(f, Z)$, "a restrição de $f$ a $Z$ tem imagem em $Z$ " como $\forall x[Z(x) \rightarrow Z(f x)], \operatorname{Inj}(f, Z)$, " $f$ é injetora em $Z$ ", como $\forall x \forall y(Z(x) \wedge Z(y) \wedge f x=f y \rightarrow x=y)$ e $S b j(f, Z)$, " $f$ é sobrejetora em $Z$ ", como $\forall x(Z(x) \rightarrow \exists y(Z(y) \wedge f y=x)$ e coloque

$$
\sigma_{<\omega}(X): \forall f(F u n(f, X) \wedge \operatorname{Inj}(f, X) \rightarrow \operatorname{Sbj}(f, X)) .
$$

Sejam $X, Y$ variáveis de predicado unárias. Definimos também

$$
\begin{gathered}
X \leq Y: \exists f \forall y(Y(y) \rightarrow \exists x(X(x) \wedge f x=y)), \\
X \subseteq Y: \forall z(X(z) \rightarrow Y(z)) .
\end{gathered}
$$

Então $M \vDash X \leq Y[A, B]$ somente no caso de a cardinalidade de $A$ for menor que a de $B$. Seja agora $\sigma_{<\aleph_{0}}(X)$ a fórmula $\sigma_{<\omega}(X)$ e defina por recursão em $n$ :

$$
\sigma_{<\aleph_{n+1}}(X): \forall Y\left(Y \subseteq X \rightarrow \sigma_{<\aleph_{n}}(Y) \vee X \leq Y\right)
$$

Definimos então $\sigma_{\aleph_{n}}(X)$ como $\sigma_{\aleph_{n+1}}(X) \wedge \neg \sigma_{\aleph_{n}}(X)$. 


\subsection{Semântica de Henkin}

A Semântica Padrão é, em algum sentido, a semântica mais natural. No entanto, há uma outra, introduzida por Henkin em Henkin (1950), que satisfaz as propriedades da Completude, Compacidade, Löwenheim-Skolem para baixo e para cima e Omissão de tipos. Ela consiste, basicamente, em se restringir o alcance das variáveis de segunda ordem, fazendoas tomar valores apenas sobre um subconjunto das partes. Vamos definir também uma semântica chamada de Semântica de Primeira Ordem, na qual não existe relação alguma entre os elementos do domínio e os quantificadores de segunda ordem. E provaremos que essas duas semânticas são essencialmente a mesma e, assim, seguirá que a relação entre os elementos do domínio com seu conjunto das partes na Semântica de Henkin é ilusória. Vamos primeiro estender a noção de $V$-estrutura para uma $V$-estrutura com tipos:

Definição 1.3.1 Um vocabulário com tipos é um vocabulário $V$ junto com um conjunto $\mathscr{T}_{V}$, chamado de conjunto dos tipos de $V$, tal que a cada símbolo $v \in V$ está associado um único tipo $\tau \in \mathscr{T}_{V}$. Se $V$ é um vocabulário com tipos, uma $V$-estrutura é um par $\langle M, I\rangle$ tal que $M$ é uma função de dominio $\mathscr{T}_{V}$ tal que $M(\tau) \neq \emptyset$ para todo $\tau \in \mathscr{T}_{V}$ e, se $v$ é um símbolo do tipo $\tau$, então $I(v)$ é uma constante, ou função, ou relação em $M(\tau)$.

A próxima definição é apenas uma variante notacional de uma estrutura para um vocabulário com tipos. Nós vamos usá-la porque ela é um pouco mais intuitiva sobre o que estamos fazendo com $L 2$.

Definição 1.3.2 Uma pré-estrutura de Henkin para uma linguagem 〈Alf, Term, Form〉 de segunda ordem é uma quádrupla $M^{H}=\left\langle M, F^{H}, R^{H}, I\right\rangle$, onde $M$ e I são como numa estrutura da semântica padrão, $F^{H}=\bigcup_{n \geq 1} F_{n}^{H}$ e $R^{H}=\bigcup_{n \geq 1} R_{n}^{H}$, de tal forma que se $f \in$ $F_{n}^{H}$, então f é uma função de $M^{n}$ em $M$ e se $R \in R_{n}^{H}$, então $R$ é uma relação n-ária em $M$. Dado uma pré-estrutura $M^{H}$ para $L$, uma atribuição s é uma função $s:$ Var $\rightarrow M \cup F^{H} \cup R^{H}$ tal que

(a) se $x \in \operatorname{Var}_{1}$, então $s(x) \in M$,

(b) se $f \in \operatorname{Var}_{2}(F, n)$, então $s(f) \in F_{n}^{H}$,

(c) se $R \in \operatorname{Var}_{2}(R, n)$, então $s(R) \in R_{n}^{H}$.

A interpretação dos termos é como na semântica padrão, com a única diferença de que se $f \in \operatorname{Var}_{2}(F, n)$, então $s(f) \in F_{n}^{H}$, obviamente. Também, a relação de satisfação é como na semântica padrão, com a única diferença que se $R \in \operatorname{Var}_{2}(R, n)$, então $s(R) \in R_{n}^{H}$.

As noções de Henkin-validade, Henkin-satisfação e Henkin-consequencia são definidas da maneira óbvia.

Observação 1.3.3 Segue que um modelo padrão é equivalente a um modelo de Henkin no qual, para cada $n, F_{n}^{H}$ é o conjunto de todas as funções de $M^{n}$ em $M$ e $R_{n}^{H}$ é $P\left(M^{n}\right)$. Tais modelos de Henkin às vezes são chamados de "full models".

A seguinte semântica se chama Semântica de Primeira Ordem e tem a idéia de "interpretar" a relação de pertinência, isto é, se $X$ for um símbolo de variável unária, vamos dizer que $M \vDash X(t)[s]$ se o par $\left\langle t^{M}[s], s(X)\right\rangle$ estiver em uma dada relação.

Definição 1.3.4 Uma estrutura de primeira ordem $M^{1}$ para uma linguagem de segunda ordem é uma quádrupla $M^{1}=\left\langle M, M_{F}, M_{R},\langle I, a, p\rangle\right\rangle$, onde $M$ e I são como numa estrutura da semântica padrão, $M_{F}=\bigcup_{n>1} M_{F, n}$ e $M_{R}=\bigcup_{n>1} M_{R, n}$. Cada $M_{F, n}$ é um conjunto de funções de $M^{n}$ em $M$ e cada $M_{R, n}$ é um conjunto de relações n-árias em $M$; a é uma 
sequencia onde $a_{n}$ é uma função, $a_{n}: M_{F, n} \times M^{n} \rightarrow M$ e p é outra sequencia, mas de relações, com $p_{n} \subseteq M^{n} \times M_{R, n}$. A idéia é que $a_{n}$ seja a interpretação de uma dada função $n$-ária em uma n-upla e, semelhantemente, $p_{n}$ seja a interpretação da relação de pertinência entre uma n-upla e uma relação n-ária. Dada uma estrutura $M^{1}$, uma atribuição s é uma função $s:$ Var $\rightarrow M \cup M_{F} \cup M_{R}$ tal que

(a) se $x \in \operatorname{Var}_{1}$, então $s(x) \in M$,

(b) se $f \in \operatorname{Var}_{2}(F, n)$, então $s(f) \in M_{F, n}$,

(c) se $R \in \operatorname{Var}_{2}(R, n)$, então $s(R) \in M_{R, n}$.

$A$ interpretação dos termos é feita como em uma estrutura qualquer (def. 1.1.8) adicionandose a cláusula

(d) se $t$ é da forma $f\left(t_{1}, t_{2}, \ldots, t_{n}\right)$ com $f \in \operatorname{Var}_{2}(F, n)$ e s é uma atribuiçãa, então $t^{M^{1}}[s]=a_{n}\left(s(f),\left\langle t_{1}^{M^{1}}, t_{2}^{M^{1}}, \ldots, t_{n}^{M^{1}}\right\rangle[s]\right)$.

A relação de satisfação é definida como em um modelo para uma fórmula de primeira ordem (def. 1.1.9), adicionando-se as cláusulas:

(e) se $\varphi$ é $X\left(t_{1}, t_{2}, \ldots, t_{n}\right)$ com $X \in \operatorname{Var}_{2}(R, n)$ e $t_{1}, t_{2}, \ldots, t_{n} \in T e r m$, então $M^{1} \vDash \varphi[s]$ se $\left\langle\left\langle t_{1}^{M^{1}}, t_{2}^{M^{1}}, \ldots, t_{n}^{M^{1}}\right\rangle, s(X)\right\rangle \in p_{n}$.

(f) se $\varphi$ é $\forall X \psi$ com $X \in \operatorname{Var}_{2}^{F} \cup \operatorname{Var}_{2}^{R}$, então $M^{1} \vDash \varphi[s]$ se para toda atribuição $s^{\prime}$ tal que se $Y \neq X$ e $s^{\prime}(Y)=s(Y)$ temos $M^{1} \vDash \psi\left[s^{\prime}\right]$.

As noções de primeira-ordem-validade, primeira-ordem-satisfação e primeira-ordem-consequencia são definidas da maneira óbvia.

Observação 1.3.5 Segue que para cada pré-estrutura de Henkin $\left\langle M, F^{H}, R^{H}, I\right\rangle$ existe uma estrutura de primeira ordem $\left\langle M, M_{F}, M_{R},\langle I, a, p\rangle\right\rangle$ tal que $M_{F}=F^{H}, M_{R}=F^{R}$, an é a função $a_{n}(w, u)=w(u)$ e $p_{n}$ é a relação de pertinência, isto é, $\langle u, v\rangle \in p_{n} \Leftrightarrow u \in v$. Assim, para cada modelo de Henkin $M^{H}$ existe um modelo de primeira ordem $M^{1}$ tal que para cada atribuição s em $M^{H}$ existe uma atribuição $s^{1}$ em $M^{1}$ tal que para toda fórmula $\varphi, M^{H} \vDash \varphi[s]$ se, e somente se, $M^{1} \vDash \varphi\left[s^{1}\right]$. Mas, além disso, vale a recíproca:

Proposição 1.3.6 Seja $M^{1}=\left\langle M, M_{F}, M_{R},\langle I\right.$, a,p $\left.\rangle\right\rangle$ uma estrutura de primeira ordem para uma linguagem de segunda ordem. Então existe uma pré-estrutura de Henkin $M^{H}=\left\langle M, F^{H}\right.$, $\left.R^{H}, I\right\rangle$ tal que para toda atribuição s em $M^{1}$ existe uma atribuição ${ }^{H}$ em $M^{H}$ tal que para toda fórmula $\varphi$ de $L 2, M^{1} \vDash \varphi[s]$ se, e somente se, $M^{H} \vDash \varphi\left[s^{H}\right]$.

Prova. Para cada $n \geq 1$, sejam

$$
\begin{aligned}
& F_{n}^{H}=\left\{f \in P\left(M^{n+1}\right): F u n(f) \wedge \exists g \in M_{F, n} \forall x_{1} \ldots x_{n}\left[f\left(x_{1}, \ldots, x_{n}\right)=a_{n}\left(g,\left\langle x_{1}, \ldots, x_{n}\right\rangle\right)\right]\right\} \\
& R_{n}^{H}=\left\{R \in P\left(M^{n}\right): \exists Q \in M_{R, n} \forall x_{1} \ldots \forall x_{n}\left(\left\langle x_{1}, \ldots, x_{n}\right\rangle \in R \leftrightarrow\left\langle\left\langle x_{1}, \ldots, x_{n}\right\rangle, Q\right\rangle \in p_{n}\right\}\right.
\end{aligned}
$$

(onde Fun $(f)$ é símbolo de predicado para " $f$ é função") e defina também $F^{H}=\bigcup_{n \geq 1} F_{n}^{H}$, $R^{H}=\bigcup_{n \geq 1} R_{n}^{H}$.

Seja agora $s$ uma atribuição em $M^{1}$. Defina $s^{H}$ do seguinte modo: se $x \in \operatorname{Var}_{1}$, então $s^{H}(x)=s(x)$; se $f \in \operatorname{Var}_{2}(F, n)$, então $s^{H}(f)(\cdot)=a_{n}(s(f), \cdot)$ e, se $R \in \operatorname{Var}_{2}(R, n)$, então $s^{H}(R)$ é a relação tal que $\left\langle x_{1}, x_{2}, \ldots, x_{n}\right\rangle \in s^{H}(R)$ se, e somente se, $\left\langle\left\langle x_{1}, x_{2}, \ldots, x_{n}\right\rangle, s(R)\right\rangle \in$ $p_{n}$. Agora a prova de que $M^{1} \vDash \varphi[s]$ se, e somente se, $M^{H} \vDash \varphi\left[s^{H}\right]$ é uma indução na complexidade de $\varphi$.

Corolário 1.3.7 Para cada $\varphi, \varphi$ é Henkin-válida se, e somente se, $\varphi$ é primeira-ordemválida e $\varphi$ é Henkin-satifazível se, e somente se, $\varphi$ é primeira-ordem-satisfazível. Para cada conjunto $\Gamma$ de fórmulas, $\Gamma$ é Henkin-satisfazível se, e somente se, $\Gamma$ é primeira-ordem satisfazivel e $\varphi$ é uma Henkin-consequencia de $\Gamma$ se, e somente se, $\varphi$ é primeira-ordem-consequencia de $\Gamma$. 
A semântica de Henkin possui uma bela teoria de modelos. Isso se deve, no fundo, por ela ser essencialmente a lógica de primeira ordem, como vamos ver no teorema de Lindström (seção 3.1). Começaremos provando que L2 pode satisfazer Completude com esta semântica; Para tanto, iremos primeiro escolher um sistema dedutivo para a Lógica de Segunda Ordem. É um problema decidir qual seria o sistema dedutivo mais apropriado para $L 2$; vamos discutir isto mais para frente.

Definição 1.3.8 A seguinte lista é a lista de axiomas do sitema dedutivo D2 da Lógica de Segunda Ordem. Sejam $\varphi, \psi$ e $\chi$ fórmulas, $x \in \operatorname{Var}_{1}, X \in \operatorname{Var}_{2}(R, n), f \in \operatorname{Var}_{2}(F, n)$, $t \in \operatorname{Term} P \in \operatorname{Var}_{2}(R, n) \cup R_{n}$ e $p \in \operatorname{Var}_{2}(F, n) \cup F_{n}$ :

1. $\varphi \rightarrow(\psi \rightarrow \varphi)$

2. $(\varphi \rightarrow \psi) \rightarrow((\varphi \rightarrow(\psi \rightarrow \chi)) \rightarrow(\varphi \rightarrow \chi))$

3. $(\neg \varphi \rightarrow \psi) \rightarrow((\neg \varphi \rightarrow \neg \psi) \rightarrow \varphi)$

4. $\forall x \varphi \rightarrow \varphi(x \mid t)$

5. $\forall X \varphi \rightarrow \varphi(X \mid P)$

6. $\forall f \varphi \rightarrow \varphi(f \mid p)$

As regras de inferência de D2 são as mesmas regras de inferência da lógica de primeira ordem (definição 1.1.5) podendo-se, é claro, usar variáveis de segunda ordem na regra de generalização.

Observação 1.3.9 Um sistema dedutivo $D$ é dito correto para uma lógica $L$ se para todos $\Gamma$, toda $\varphi$, se $\Gamma \vdash_{D} \varphi$ então $\Gamma \vDash_{L} \varphi$. Pré-estruturas de Henkin não são corretos para este sistema: suponha que $R_{1}^{H}=\{M\}$ e que $P$ seja um simbolo de predicado com $I(P) \neq M$; então $M \vDash \forall X \forall x X(x)$ mas $M \not \models \forall x P(x)$, e assim não satisfaz o axioma 5. Dado esse inconveniente, definimos uma estrutura de Henkin, à qual vai seguir imediatamente a correção.

Definição 1.3.10 Uma estrutura de Henkin é uma pré-estrutura de Henkin correta para o sistema dedutivo D2.

A partir de agora, consideraremos $M, s$ um modelo de Hekin apenas se $M$ for uma estrutura de Henkin.

Proposição 1.3.11 D2 é correto para L2 com a semântica das estruturas de Henkin (e consequentemente, para a semântica padrão).

Observação 1.3.12 Em linguagens de segunda ordem podemos definir a igualdade de objetos em termos puramente lógicos, diferentemente do que acontece em primeira ordem, onde é necessário um simbolo para igualdade e axiomas para descrever seu comportamento. Se $s, t \in$ Term, definimos:

$$
s=t: \forall X(X(s) \leftrightarrow X(t)),
$$

onde $X \in \operatorname{Var}_{2}^{R_{1}}$, isto é, $s=t$ se e só se todo conjunto que contiver s contém $t$ e vice-versa. A igualdade entre relações e funções é definida usando-se o "princípio da extensionalidade": se $P, Q \in R_{n} \cup \operatorname{Var}_{2}(R, n)$ e $f, g \in F_{n} \cup \operatorname{Var}_{2}(F, n)$, então

$$
\begin{gathered}
P=Q: \forall x_{1} \forall x_{2} \ldots \forall x_{n}\left(P\left(x_{1}, x_{2}, \ldots, x_{n}\right) \leftrightarrow Q\left(x_{1}, x_{2}, \ldots, x_{n}\right)\right) \\
f=g: \forall x_{1} \forall x_{2} \ldots \forall x_{n}\left(f\left(x_{1}, x_{2}, \ldots, x_{n}\right)=g\left(x_{1}, x_{2}, \ldots, x_{n}\right)\right)
\end{gathered}
$$


A partir destas definições, pode-se provar que = é uma relação de equivalência. No entanto, veja que essa definição de igualdade não faz distinção entre dois elementos do dominio que não sejam separados por conjuntos; isto é, se tivermos $a \neq b$ mas em $R_{1}^{H}$ não houver dois conjuntos $A$ e $B$ tais que $a \in A-B$ e $b \in B-A$, e se $s$ for uma atribuição com $s(x)=a$ e $s(y)=b$, então teremos $M^{H} \vDash x=y[s]$. No entanto, isto não tem maior importância; se muito incomodados, podemos tomar como dominio a classe de equivalência dos elementos que $M^{H}$ garantir que são iguais. Modelos de Henkin que tem a propriedade de garantir que $M^{H} \vDash a=b$ somente se a for $b$ são chamados de "identity models".

Vamos passar agora à demonstração do teorema da Completude com semântica de Henkin. Estaremos usando o "método das constantes", introduzido por Henkin em Henkin (1949) para provar a completude do chamado "Cálculo Funcional de Primeira Ordem", e usado depois pelo mesmo autor em Henkin (1950) para provar a Completude da "Teoria dos Tipos" em geral. Contudo o faremos, é claro, na nossa linguagem de segunda ordem. Uma tradução de um sistema para outro pode ser encontrado em Gallin (1975).

Teorema 1.3.13 Seja $\Gamma \cup\{\varphi\}$ um conjunto de fórmulas de segunda ordem consistente em relação a D2. Se todo modelo de Henkin que é modelo de $\Gamma$ é modelo de $\varphi$, então $\Gamma \vdash_{D 2} \varphi .^{11}$

Prova. A demostração é feita em várias etapas. O primeiro item a ser notado é que

Lema 1.3.14 Podemos supor que $\Gamma$ é um conjunto de sentenças. Mais precisamente, se $V L(\varphi)=\left\{x_{1}, \ldots, x_{n}\right\}$, e se $M^{\prime}$ é um $\left(V \cup\left\{c_{1}, \ldots, c_{n}\right\}\right)$-modelo tal que $M^{\prime} \uparrow V=M$, então

$$
M \vDash \varphi[s] \Leftrightarrow M^{\prime} \vDash \varphi\left(x_{1}\left|c_{1}, \ldots, x_{n}\right| c_{n}\right),
$$

onde $c_{i}^{M^{\prime}}=s\left(x_{i}\right), 1 \leq i \leq n$.

A estratégia agora é a seguinte: dado $\Sigma$ conjunto de sentenças consistente, mostramos que tem um modelo. A conclusão do teorema vai seguir contrapositivamente: se for o caso de $\Gamma \nvdash \varphi$, então $\Gamma \cup\{\neg \varphi\}$ é consistente (é um lema), e assim $\Gamma \cup\{\neg \varphi\}$ tem modelo e portanto $\Gamma \not \models \varphi$.

Suponha então que $\Sigma \subseteq L 2(V)$. Vamos construir uma sequência $\Sigma_{0}=\Sigma \subseteq \Sigma_{1} \subseteq \Sigma_{2} \subseteq \ldots$ num vocabulário $V^{\prime}$ contendo $V$ tal que (1) cada $\Sigma_{i}$ é consistente, (2) se $\exists x \varphi \in \Sigma_{i}$ (isto é, se $\neg \forall x \neg \varphi \in \Sigma$ ), onde $x$ é uma variável de primeira ou segunda ordem, então existe um símbolo $c$ que não ocorre em $\Sigma_{j}, j<i$, do tipo correspondente à variável, tal que $\varphi(x \mid c) \in \Sigma_{i}$ e (3) $\bigcup_{i>0} \Sigma_{i}$ é maximal consistente em $L 2\left(V^{\prime}\right)$.

Sejam $C^{\prime}=\left\{c_{n}: n \in \omega\right\}, F^{\prime}=\left\{f_{n, m}: n, m \in \omega\right\}$ e $R^{\prime}=\left\{R_{n, m}: n, m \in \omega\right\}$ conjuntos de novos símbolos de constantes, funções e relações, respectivamente, onde o segundo índice indica a aridade. Considere $V^{\prime}=V \cup C^{\prime} \cup F^{\prime} \cup R^{\prime}$ e seja $\sigma_{n}$ uma enumeração de $L 2\left(V^{\prime}\right)$. Defina $\Sigma_{n+1}$ segundo as 3 cláusulas seguintes:

- se $\Sigma_{n} \cup\left\{\sigma_{n}\right\}$ é inconsistente, então $\Sigma_{n+1}=\Sigma_{n}$.

- se $\Sigma_{n} \cup\left\{\sigma_{n}\right\}$ é consistente e $\sigma_{n}$ não é da forma $\exists x \varphi$, então $\Sigma_{n+1}=\Sigma_{n} \cup\left\{\sigma_{n}\right\}$.

- se $\Sigma_{n} \cup\left\{\sigma_{n}\right\}$ é consistente e $\sigma_{n}$ é da forma $\exists x \varphi$, considere se $x$ é uma variável de primeira ordem, de função ou relação, e tome o primeiro símbolo do tipo da variável (de mesma aridade da variável, se for o caso) que não ocorre em $\Sigma_{n} \cup\left\{\sigma_{n}\right\}$; digamos que seja c. Defina então $\Sigma_{n+1}=\Sigma_{n} \cup\{\exists x \varphi, \varphi(x \mid c)\}$; afirmamos que $\Sigma_{n+1}$ é consistente. De fato, considere o seguinte lema:

\footnotetext{
${ }^{11} \mathrm{Na}$ verdade, estaremos usando mais uma hipótese: a de que o vocabulário usado nas fórmulas de $\Gamma$ é um conjunto enumerável. Como para $L 1$, essa hipótese pode ser suprimida; não o faremos aqui, contudo. A alteração na demonstração é a mesma que precisa ser feita para $L 1$.
} 
Lema 1.3.15 Se $\Gamma \vdash \varphi(x \mid c)$ e c não ocorre em nunhuma fórmula de $\Gamma$, então $\Gamma \vdash \forall x \varphi$.

Logo, se $\Sigma_{n+1}$ fosse inconsistente, $\Sigma_{n}, \exists x \varphi \vdash \neg \varphi(x \mid c)$, donde $\Sigma_{n}, \exists x \varphi \vdash \forall x \neg \varphi$, contrariando a hipótese de $\Sigma_{n} \cup\left\{\sigma_{n}\right\}$ ser consistente.

Falta provar que $\Sigma_{\omega}=\bigcup_{n \geq 0} \Sigma_{n}$ é maximal consistente. A consistência é clara. Se $\sigma \in$ $L 2\left(V^{\prime}\right)-\Sigma_{\omega}$, então como $\sigma$ é $\sigma_{n}$ para algum $n$, temos $\Sigma_{n} \cup\left\{\sigma_{n}\right\}$ inconsistente (pois no $n$-ésimo passo $\sigma_{n}$ não foi adicionado a $\left.\Sigma_{n+1}\right)$, donde $\Sigma_{\omega} \cup\{\sigma\}$ é inconsistente.

Construção do modelo. Considere a seguinte relação de equivalência entre símbolos de constantes: $c \sim d \Leftrightarrow \Sigma_{\omega} \vdash c=d$. Defina $\left|M^{H}\right|=\left\{[c]: c \in C^{\prime}\right\}$, onde $[c]$ é a classe de equivalência de $c$ em relação a $\sim$ (para ver que $\sim$ é uma relação de equivalência, mostre que as seguintes fórmulas são teoremas: $\forall x(x=x), \forall x \forall y(x=y \rightarrow y=x)$ e $\forall x \forall y \forall z(x=y \wedge y=$ $z \rightarrow x=z$ ), segundo a notação 1.3.12), e coloque $I(c)=[c]$. Se $f \in F_{n}^{\prime} \cup F_{n}$, seja $I(f)=$ $\left\{\left\langle\left[c_{1}\right], \ldots,\left[c_{n+1}\right]\right\rangle: \Sigma_{\omega} \vdash f\left(c_{1}, \ldots, c_{n}\right)=c_{n+1}\right\}$ e coloque ainda $F_{n}^{H}=\left\{I(f): f \in F_{n}^{\prime} \cup F_{n}\right\}$ e $F^{H}=\bigcup_{n \geq 0} F_{n}^{H}$. Como todo termo $t$ sem ocorrência de váriáveis tem uma interpretação $t^{M^{H}}$, faz sentido definir para $P \in R_{n}^{\prime} \cup R_{n}$ a interpretação $I(P)=\left\{\left\langle\left[t_{1}^{M^{H}}\right], \ldots,\left[t_{n}^{M^{H}}\right]\right\rangle: \Sigma_{\omega} \vdash\right.$ $\left.P\left(t_{1}, \ldots, t_{n}\right)\right\}, R_{n}^{H}=\left\{I(P): P \in R_{n}^{\prime} \cup R_{n}\right\}$ e $R^{H}=\bigcup_{n \geq 0} R_{n}^{H}$. Vamos mostrar agora que $M^{H}=\left\langle\left|M^{H}\right|, F^{H}, R^{H}, I\right\rangle$ é um modelo de Henkin de $\Sigma$.

Precisamos mostrar que se $f \in F_{n}^{\prime}$, então $I(f)$ é uma função de $D^{n}$ em $D$. Suponha então que $\left\langle\left[c_{1}\right], \ldots,\left[c_{n}\right],[d]\right\rangle \in I(f)$ e $\left\langle\left[c_{1}\right], \ldots,\left[c_{n}\right],\left[d^{\prime}\right]\right\rangle \in I(f)$. Por definição de $I(f)$, temos que $\Sigma_{\omega} \vdash f\left(c_{1}, \ldots, c_{n}\right)=d$ e $\Sigma_{\omega} \vdash f\left(c_{1}, \ldots, c_{n}\right)=d^{\prime}$; segue da simetria e da transitividade da igualdade que $\Sigma_{\omega} \vdash d=d^{\prime}$, donde $[d]=\left[d^{\prime}\right]$.

Seja agora $\varphi \in \Sigma$. Vamos mostrar por indução na complexidade de $\varphi$ que $M \vDash \varphi$. Se $\varphi$ é atômica, então $\varphi$ é $P\left(t_{1}, \ldots, t_{n}\right)$, onde $t_{1}, \ldots, t_{n}$ são termos sem variáveis e $P \in R_{n}$. Como $\Sigma \subseteq \Sigma_{\omega}$, temos $\Sigma_{\omega} \vdash P\left(t_{1}, \ldots, t_{n}\right)$ e assim por definição de $I(P),\left\langle\left[t_{1}^{M^{H}}\right], \ldots,\left[t_{n}^{M^{H}}\right]\right\rangle \in I(P)$.

Se $\varphi$ é $\neg \psi$, então $M \vDash \neg \psi \Leftrightarrow M \not \models \psi \Leftrightarrow \Sigma_{\omega} \nvdash \psi$ (caso contrário $M \vDash \psi$ por hipótese de indução) $\Leftrightarrow \Sigma_{\omega} \vdash \neg \psi$, já que $\Sigma_{\omega}$ é maximal consistente.

Se $\varphi$ é $\psi \rightarrow \chi$, é direto.

Se $\varphi$ é $\forall x \psi$, suponha que $x \in \operatorname{Var}_{1}$. Então para todo símbolo de constante de indivíduo temos $\Sigma_{\omega} \vdash \psi(x \mid c)$; como todo $d \in\left|M^{H}\right|$ é a classe de equivalência de algum $c, M \vDash \forall x \psi$ (a prova é uma indução na complexidade de $\psi$ ).

Se $\varphi$ é $\forall X \psi$ com $X \in \operatorname{Var}_{2}(R, n)$, então para todo símbolo de relação $n$-ária $P$, temos $\Sigma_{\omega} \vdash \psi(X \mid P)$. Como todo $r \in R_{n}^{H}$ é a interpretação de algum símbolo de relação $n$-ária (por construção de $\left.R_{n}^{H}\right), M \vDash \psi[X \mid r]$ para todo $r \Leftrightarrow M \vDash \forall X \psi$. O caso em que $\varphi$ é da forma $\forall f \psi$ é análogo.

Concluímos que $\left\langle M, R^{H}, F^{H}, I\right\rangle$ é um modelo de Henkin de $\Sigma$.

Observação 1.3.16 Observe que poderíamos ter enunciado o teorema para pré-estruturas de Henkin, isto é, para estruturas $M^{H}$ nas quais não são válidos os axiomas 5 e 6 da lista em 1.3 .8 (como toda estrutura de Henkin é uma pré-estrutura de Henkin, teorias consistentes em tal sistema tem modelo); neste caso, se quiséssemos ter correção, teríamos que retirar estes axiomas do sistema dedutivo. Semelhantemente, poderíamos ter adicionado à lista de axiomas de D2 sentenças que são válidas no modelo que construímos acima. Isso será discutido mais adiante, na seção 1.4.

Corolário 1.3.17 Semântica de Henkin satisfaz Compacidade (1.1.15).

Prova. A prova é a mesma de $L 1$. Suponha que todo subconjunto finito de $\Gamma$ tem modelo. Como D2 é correto, $\Gamma$ é consistente; por completude, tem modelo. 
Na verdade, a semântica de Henkin irá satisfazer todas as propriedades 1.1.14 a 1.1.19. Para mostrarmos Löwenheim-Skolem, contudo, vamos precisar da noção de submodelo para modelos de Henkin, que difere um pouco da usual por conta de uma estrutura de Henkin na verdade ser uma estrutura para um vocabulário com tipos. Desta forma, as propriedades de Löwenheim-Skolem também precisam ser modificadas, mas não essencialmente:

Definição 1.3.18 $N a$ semântica de Henkin, $M^{\prime}=\left\langle\left|M^{\prime}\right|, F^{H^{\prime}}, R^{H^{\prime}}, I^{\prime}\right\rangle$ é uma subestrutura de $M=\left\langle|M|, F^{H}, R^{H}, I\right\rangle$ se

(a) $\left|M^{\prime}\right| \subseteq|M|$,

(b) para cada $f^{\prime} \in F_{n}^{H^{\prime}}$ existe $f \in F_{n}^{H}$ tal que $f^{\prime}=f \uparrow\left|M^{\prime}\right|^{n}$,

(c) para cada $P^{\prime} \in R_{n}^{H^{\prime}}$ existe $P \in R_{n}^{H}$ tal que $P^{\prime}=P \cap\left|M^{\prime}\right|^{n}$ e $I^{\prime}$ é como na definição de subestrutura, definição 1.1.16. Neste caso, existem funções $k$, chamadas de correspondências entre $M^{\prime}$ e $M$, tais que

(i) $f \in F_{n}^{H^{\prime}} \Rightarrow k(f) \in F_{n}^{H}$ e $f=k(f) \uparrow\left|M^{\prime}\right|^{n}$,

(ii) $P \in R_{n}^{H^{\prime}} \Rightarrow k(P) \in R_{n}^{H}$ e $P=k(P) \cap\left|M^{\prime}\right|^{n}$.

Dada uma atribuição s em $M^{\prime}$, para cada correspondência $k$ definimos uma atribuição $s^{k}$ em $M$ do seguinte modo: se $x \in \operatorname{Var}_{1}$, então $s^{k}(x)=s(x)$, se $f \in \operatorname{Var}_{2}(F, n)$, então $s^{k}(f)=k(s(f))$ e se $X \in \operatorname{Var}_{2}(R, n)$, ent $\tilde{a} o s^{k}(X)=k(s(X))$.

Teorema 1.3.19 Seja $M=\left\langle|M|, F^{H}, R^{H}, I\right)$ uma estrutura de Henkin. Então existe uma subestrutura $M^{\prime}=\left\langle\left|M^{\prime}\right|, F^{H^{\prime}}, R^{H^{\prime}}, I^{\prime}\right\rangle$ de $M$ e uma correspondência $k$ entre $M^{\prime}$ e $M$ tais que

(1) $M^{\prime}$ e cada $F_{n}^{H^{\prime}}, R_{n}^{H^{\prime}}$ tem cardinalidade no máximo a do vocabulário de $M$ ou infinita enumerável, a que for maior $e$

(2) $M^{\prime} \vDash \varphi[s] \Leftrightarrow M \vDash\left[s^{k}\right]$.

Em particular, se $\Gamma$ é um conjunto de fórmulas que tem modelo, então $\Gamma$ tem modelo de cardinalidade no máximo a do vocabulário de $\Gamma$ ou infinita enumerável, a que for maior.

Prova. Considere a função e definida assim: para cada fórmula da forma $\exists x \varphi$ tal que $M \vDash \exists x \varphi$ (com $x$ de primeira ou segunda ordem), seja $e(\exists x \varphi)$ um $a \in|M|$ (ou $a \in F^{H}$, ou $a \in R^{H}$, conforme o caso) tal que $M \vDash \varphi[x \mid a]$. Seja $V=C \cup F \cup R$ o vocabulário de $M$ e defina

$$
\begin{aligned}
M_{0}^{\prime}= & \{I(c): c \in C\} \cup\left\{\operatorname{todos} e(\exists x \varphi), x \in \operatorname{Var}_{1}\right\} \\
M_{n+1}^{\prime}= & M_{n} \cup\left\{I(f)\left(a_{1}, \ldots, a_{m}\right): a_{1}, \ldots, a_{m} \in M_{n}^{\prime}, f \in F_{n} \text { e } m \in \omega\right\} \\
& \cup\left\{\operatorname{todos} e(\exists f \varphi)\left(a_{1}, \ldots, a_{m}\right): a_{1}, \ldots, a_{m} \in M_{n}^{\prime}, f \in \operatorname{Var}_{2}(F, n) \text { e } m \in \omega\right\}
\end{aligned}
$$

e $M^{\prime}=\bigcup M_{n}^{\prime}$. Coloque agora, para cada $n \in \omega$,

$$
\begin{aligned}
F_{n}^{H^{\prime}}= & \left\{I(f) \uparrow\left(M^{\prime}\right)^{n}: f \in F_{n}\right\} \\
& \cup\left\{\operatorname{todos} e(\exists f \varphi) \uparrow\left(M^{\prime}\right)^{n}: f \in \operatorname{Var}_{2}(F, n)\right\} \\
R_{n}^{H^{\prime}}= & \left\{I(P) \cap\left(M^{\prime}\right)^{n}: P \in R_{n}\right\} \\
& \cup\left\{\operatorname{todos} e(\exists X \varphi) \cap\left(M^{\prime}\right)^{n}: X \in \operatorname{Var}_{2}(R, n)\right\},
\end{aligned}
$$

e sejam $F^{H^{\prime}}=\bigcup F_{n}^{H^{\prime}}, R^{H^{\prime}}=\bigcup R_{n}^{H^{\prime}}$. Definindo agora $I^{\prime}$ da maneira óbvia, $M^{\prime}$ é uma subsestrutura de $M$ satisfazendo (1), já que cada função em $F^{H}$ é fechada em $M^{\prime}$ : de fato, se $g \in F_{n}^{H^{\prime}}$, então $g$ é da forma $I(f) \uparrow\left(M^{\prime}\right)^{n}$ ou $e(\exists f \varphi) \uparrow\left(M^{\prime}\right)^{n}$, para algum $n \in \omega$; dados então $a_{1}, \ldots, a_{n} \in M^{\prime}$, seja $k=\min \left\{m: a_{1}, \ldots, a_{n} \in M_{m}^{\prime}\right\}$. Então $g\left(a_{1}, \ldots, a_{n}\right) \in M_{k+1}^{\prime} \subseteq M^{\prime}$.

Para provar (2), considere uma correspondência $k$ entre $M^{\prime}$ e $M$, que existe por construção de $M^{\prime}$. Observe primeiro que se $t$ é um termo qualquer e s uma atribuição em $M^{\prime}$, então $t^{M^{\prime}}[s]=t^{M}\left[s^{k}\right]$ (a prova é uma indução na complexidade de $t$ ). Faremos uma indução na complexidade de $\varphi$ : se é atômica $X\left(t_{1}, \ldots, t_{n}\right) \operatorname{com} X \in \operatorname{Var}_{2}(F, n)$, então 


$$
\begin{aligned}
M^{\prime} \vDash X\left(t_{1}, \ldots, t_{n}\right)[s] & \Leftrightarrow\left\langle t_{1}^{M^{\prime}}[s], \ldots, t_{n}^{M^{\prime}}[s]\right\rangle \in s(X) \\
& \left.\Leftrightarrow\left\langle t_{1}^{M}[s], \ldots, t_{n}^{M}[s]\right\rangle \in s^{k}(X) \text { (por definição de } s^{k}\right) \\
& \Leftrightarrow M \vDash X\left(t_{1}, \ldots, t_{n}\right)\left[s^{k}\right] .
\end{aligned}
$$

Se $\varphi$ é $P\left(t_{1}, \ldots, t_{n}\right)$ com $P$ um símbolo de relação, é imediato. Os casos em que $\varphi$ é da forma $\psi \wedge \chi \mathrm{e} \neg \psi$ seguem diretamente da hipótese de indução. Se $\varphi$ é $\exists x \psi, x \in \operatorname{Var}_{1}$, considere $s(x \mid a)$, a atribuição obtida de $s$ trocando seu valor na variável $x$ por $a$; então, para algum $a \in M^{\prime}$, temos

$$
\begin{aligned}
M^{\prime} \vDash \exists x \psi[s] & \Leftrightarrow M^{\prime} \vDash \psi[s(x \mid a)] \\
& \Leftrightarrow M \vDash \psi\left[s^{k}(x \mid a)\right] \text { (pela hipótese de indução) } \\
& \Rightarrow M \vDash \exists x \psi\left[s^{k}\right] .
\end{aligned}
$$

Para a volta, se $M \vDash \psi\left[s^{k}\right]$, então $M \vDash \psi\left[s^{k}(x \mid e(\exists x \psi))\right]$, e assim $M \vDash \psi\left[s^{k}(x \mid a)\right]$ para algum $a \in M^{\prime}$, que por hipótese de indução é equivalente a $M^{\prime} \vDash \psi[s(x \mid a)]$, donde $M^{\prime} \vDash \exists x \psi[s]$, como queríamos.

Os casos restantes são semelhantes a este último.

Teorema 1.3.20 Semântica de Henkin satisfaz Omissão de tipos (1.1.19), isto é: suponha que $\Gamma$ seja um $(n+m+k)$-tipo isolado sobre $T$, com $V L(\Gamma) \subseteq\left\{x_{1}, \ldots, x_{n}, X_{1}, \ldots, X_{m}, f_{1}, \ldots, f_{k}\right\}$. Então existe um modelo $M, s$ enumerável tal que $M \vDash T$ mas $M \not \models \Gamma[s]$.

Prova. Suponha sem perda de generalidade que as variáveis livres de $\Gamma$ sejam apenas de primeira ordem e de relação, de tal forma que $\Gamma$ seja um $(n+m)$-tipo sobre $T$, e seja $K=\max \{n, m\}$. Sejam $c_{2 i}, P_{2 i+1}, i \geq 0$, novos símbolos de constante e de relação, respectivamente, e $V^{\prime}=V \cup\left\{c_{2 i}, P_{2 i+1}: i \geq 0\right\}$. Seja também $\sigma_{i}$ uma enumeração de $L 2\left(V^{\prime}\right)$. Vamos construir uma sequencia

$$
T=T_{0} \subseteq T_{1} \subseteq T_{2} \subseteq \ldots \subseteq T_{i} \subseteq \ldots
$$

tal que, para cada $i \in \omega$,

(1) $T_{i}$ é um conjunto de sentenças consistente de $L 2\left(V^{\prime}\right)$ que é uma extensão finita de $T$.

(2) $\sigma_{i} \in T_{i+1}$ ou $\neg \sigma_{i} \in T_{i+1}$.

(3) Se $\sigma_{i}$ for da forma $\exists x \psi$ (onde $x$ pode ser uma variável de primeira ou segunda ordem) e $\sigma_{i} \in T_{i+1}$, então $\psi\left(x \mid v_{p}\right) \in T_{i+1}$, onde $v_{p}$ é o primeiro símbolo de $V^{\prime}$ do mesmo tipo que a variável $x$ que não ocorre em $T_{i}$ ou $\sigma_{i}$.

(4) Existe $\psi \in \Gamma$ tal que $\neg \psi\left(c_{2 i}, \ldots, c_{2(K+i)}, P_{2 i+1}, \ldots, P_{2(K+i)+1}\right) \in T_{2 i+1}$.

Suponha que $T_{2 i}=T \cup\left\{\theta_{1}, \ldots, \theta_{r}\right\}, r \geq 1$, e que $\theta$ seja a conjunção $\theta_{1} \wedge \ldots \wedge \theta_{r}$. Sejam $c_{0}, \ldots, c_{j}, P_{1}, \ldots, P_{j}$ contendo todos os símbolos de $V^{\prime}-V$ que ocorrem em $\theta$. Forme $\bar{\theta}$ uma fórmula sem símbolos de $V^{\prime}-V$ a partir de $\theta$ da seguinte forma: substitua cada símbolo $c_{k}$ ou $P_{k}$ pela variável $x_{k}$ ou $X_{k}$, conforme o caso, renomeando variáveis ligadas se necessário, e depois prefixe $\exists x_{k}$ ou $\exists X_{k}$, se $k \neq 2 i, \ldots, 2(K+i)+1$, conforme o caso. Então $\bar{\theta}$ é consistente com $T$ e, como $\Gamma$ é isolado, existe $\psi \in \Gamma$ tal que $\bar{\theta} \wedge \neg \psi$ é consistente com $T$. Note que $V L(\bar{\theta} \wedge \psi) \subseteq\left\{x_{2 i}, \ldots, x_{2(K+i)}, X_{2 i+1}, \ldots, X_{2(K+i)+1}\right\}$. Coloque $\neg \psi\left(c_{2 i}, \ldots, c_{2(K+i)}\right.$, $\left.P_{2 i+1}, \ldots, P_{2(K+i)+1}\right)$ em $T_{2 i+1}$. Assim, (4) se verifica.

As propriedades (1), (2) e (3) podem ser verificadas como no teorema da completude, 1.3.13.

Seja agora $T_{\omega}=\bigcup_{n \in \omega} T_{n}$. Seja $M^{H}$ um modelo enumerável de $T_{\omega}$; então, por (1), (2) e (3), segue por indução na complexidade de $\varphi$ que $M^{H} \vDash \varphi \Leftrightarrow T_{\omega} \vdash \varphi$. Finalmente, por (4) existe uma atribuição $s$ tal que $M^{H} \not \models \Gamma[s]$. 


\subsection{Sistemas Dedutivos e L2}

Pelo corolário 1.2.9, não existe um sistema dedutivo completo para lógica de Segunda Ordem com a semântica padrão mas, como vimos pelo teorema 1.3.13, é possível definir uma semântica com uma classe mais vasta de estruturas para a qual existe um tal sistema. Na observação 1.3.16 chamamos a atenção para o fato de que o sistema que usamos podia ser alterado para um com menos axiomas; a seguir, vamos mostrar que ele também pode ser alterado para se ter mais axiomas.

Inicialmente, temos uma razão óbvia para querer mais axiomas: quanto mais fórmulas tivermos por válidas, tanto maior é o nosso poder de demonstração, isto é, com um sistema com mais axiomas lógicos, podemos ter mais fórmulas que são demonstráveis e válidas em todas as estruturas, o que parece algo atraente para a prática matemática comum. Porém aqui podemos entrar numa questão que é, até mesmo, filosófica: será que queremos aumentar nossas pressuposições a respeito de sistemas de segunda ordem? Dependendo de quais axiomas escolhermos, podemos acabar, implicitamente, exigindo mais do que gostaríamos que fosse verdadeiro. Aumentar o número de fórmulas demonstráveis aumenta a probabilidade não apenas de se poder provar uma contradição, mas também de algo que possa incomodar nossa intuição sobre o objeto que estamos estudando. Essa questão pode parecer tola, por não ser um problema matemático propriamente dito, mas ao tratarmos de Lógica de Segunda Ordem estamos também entrando, por assim dizer, no reino da Teoria dos Conjuntos, já que as variáveis de segunda ordem tomam valores no conjunto das partes das estruturas. Já há muito tempo que se notou uma espécie de dependência mútua entre o axioma das partes e o axioma da especificação ${ }^{12}$; uma referência onde pode-se achar uma discussão sobre este tópico é Fraenkel et al. (1973).

Olhando do ponto de vista das semânticas que estamos adotando, aumentar o número de axiomas também tem o seguinte efeito: faz com que se diminua a quantidade de modelos admissíveis, e assim aproxima a classe de modelos à Semântica Padrão. É claro que não é possível, no entanto, estreitarmos a classe até a Semântica Padrão, pois por 1.2.9 esta não é axiomatizável.

O primeiro axioma que vamos considerar é o seguinte axioma (impredicativo) da compreensão:

Definição 1.4.1 Axioma da Compreensão. Se $X \in \operatorname{Var}_{2}(R, n)$ não ocorre livre em $\varphi$ e se $x_{1}, x_{2}, \ldots, x_{n}$ são as únicas variáveis que ocorrem livres em $\varphi$ e se são todas de primeira ordem, então

$$
\exists X \forall x_{1} \forall x_{2} \ldots \forall x_{n}\left(X\left(x_{1}, x_{2}, \ldots, x_{n}\right) \leftrightarrow \varphi\right)
$$

é um axioma de compreensão.

Este axioma diz que para cada fórmula $\varphi$ com apenas variáveis livres de primeira ordem existe uma relação de objetos de primeira ordem que é a "extensão" de $\varphi$. Observe que ele permite a formação de vários conjuntos (isto é, relações unárias) que são, a princípio, comuns à Teoria de Conjuntos, tais como o de par não-ordenado, a união de um conjunto, etc.

Proposição 1.4.2 Seja D2' o sistema dedutivo obtido por se adicionar o Axioma da Compreensão à lista de axiomas de D2. Então o teorema da completude (1.3.13) continua válido se trocarmos $D 2$ por $D 2^{\prime}$.

\footnotetext{
${ }^{12}$ Que também é chamado às vezes de axioma da compreensão. No nosso trabalho será chamado sempre de axioma da especificação, pois reservaremos o nome de Axioma da Compreensão para outro axioma.
} 
Prova. Em primeiro lugar, note que a lista de axiomas de $D 2^{\prime}$ é consistente, já que seus axiomas são válidos na semântica padrão e porque $D 2^{\prime}$ é um sistema correto para esta mesma semântica. Considere agora todas as construções do teorema da completude (tais como $\Sigma_{\omega}$ e $M^{H}$ ) e seja $\varphi$ tal que $V L(\varphi) \subseteq\left\{x_{1}, \ldots, x_{n}\right\}$; então

$$
\Sigma_{\omega} \vdash \exists X \forall x_{1}, \ldots \forall x_{n}\left(X\left(x_{1}, \ldots x_{n}\right) \leftrightarrow \varphi\right),
$$

já que o Axioma da Compreensão é agora um axioma do sistema dedutivo em questão. Ao construirmos $\Sigma_{\omega}$, adicionamos um símbolo $P$ ao vocabulario para cada fórmula do tipo $\exists X \psi$ que $\Sigma_{\omega}$ provava e ainda garantimos que, neste caso, $\psi(X \mid P) \in \Sigma_{\omega} ; \log 0$

$$
\Sigma_{\omega} \vdash \forall x_{1}, \ldots \forall x_{n}\left(P\left(x_{1}, \ldots x_{n}\right) \leftrightarrow \varphi\right)
$$

e, consequentemente, $\Sigma_{\omega} \vdash P\left(t_{1}, \ldots, t_{n}\right) \leftrightarrow \varphi\left(t_{1}, \ldots, t_{n}\right)$, para todos os termos $t_{1}, \ldots, t_{n}$ do vocabulário $V^{\prime}$. Agora, como cada elemento de $\left|M^{H}\right|$ tem um nome neste vocabulário, temos que para todos $a_{1}, \ldots, a_{n} \in\left|M^{H}\right|$,

$$
\left\langle a_{1}, \ldots, a_{n}\right\rangle \in P^{M^{H}} \Leftrightarrow M^{H} \vDash \varphi\left[a_{1}, \ldots, a_{n}\right] .
$$

Finalmente, por construção de $M^{H}, P^{M^{H}} \in R_{n}^{H} \subseteq R^{H}$, e assim podemos tomar uma atribuição $s$ tal que $s(X)=P^{M^{H}}$, e neste caso $M^{H} \vDash \forall x_{1} \ldots \forall x_{n}\left(X\left(x_{1} \ldots, x_{n}\right) \leftrightarrow \varphi\right)[s]$, donde segue que $M^{H} \vDash \exists X \forall x_{1} \ldots \forall x_{n}\left(X\left(x_{1}, \ldots x_{n}\right) \leftrightarrow \varphi\right)$. Assim, $\Sigma$ tem modelo, donde segue a tese.

Corolário 1.4.3 Se o Axioma da Compreensão for adicionado à lista de axiomas de D2 ainda teremos Correção e Completude para L2.

Um motivo final para se usar este axioma se relaciona à area da análise: chame um modelo de Henkin de $\omega$-modelo se seu domínio for $\omega$ e se seu vocabulário for o mesmo de $N$, com a interpretação usual. Enderton (2000) prova que se dois $\omega$-modelos tem o mesmo conjunto de relações unárias, então eles são iguais e, assim, dois $\omega$-modelos diferem apenas no conjunto das partes de $\omega$ (o argumento é que relações $n$-árias podem ser codificadas recursivamente em relações unárias. Como a codificação é recursiva, a relação unária é representável por uma fórmula da teoria dos números de primeira ordem; usa-se então o axioma da compreensão para garantir a sua existência. Dado então que esta relação existe no outro modelo, construímos a relação $n$-ária correspondente de modo semelhante). Esta abordagem da análise ${ }^{13}$ é motivada por não haver, digamos assim, um claro entendimento do conjunto $P(\omega)$.

Agora, nossa lista de axiomas pode continar crescendo: considere ainda uma forma do axioma da escolha:

Definição 1.4.4 Axioma Universal da Escolha. ${ }^{14}$ Suponha que $X \in \operatorname{Var}_{2}^{R_{n+1}}$ e que $f \in \operatorname{Var}_{2}^{F_{n}}$. Então

$$
\forall X\left[\forall x_{1} \ldots \forall x_{n} \exists y X\left(x_{1}, \ldots, x_{n}, y\right) \rightarrow \exists f \forall x_{1} \ldots \forall x_{n} X\left(x_{1}, \ldots, x_{n}, f\left(x_{1}, \ldots, x_{n}\right)\right)\right],
$$

é um axioma universal de escolha.

\footnotetext{
${ }^{13}$ Este nome para a Teoria dos Números de Segunda Ordem se deve ao fato de existir uma correspondência entre conjuntos de números naturais e números reais.

${ }^{14} \mathrm{Na}$ literatura, este axioma é normalmente chamado apenas de Axioma da Escolha. Decidimos chamá-lo de Axioma Universal da Escolha para não houver confusão entre este axioma e o Axioma da Escolha de $Z F C$.
} 
Este axioma diz que se para cada sequência $x_{1}, \ldots, x_{n}$ existe um $y$ tal que $X\left(x_{1}, \ldots, x_{n}, y\right)$, então existe uma função $f$ que escolhe um tal $y$.

Proposição 1.4.5 Seja D2" o sistema dedutivo obtido por se adicionar o Axioma Universal da Escolha à lista de axiomas de D2. Então o teorema da completude (1.3.13) continua válido se trocarmos D2" por D2.

Prova. Provamos de modo semelhante a 1.4.2. Seja $P^{M^{H}} \in R_{n+1}^{H}$ tal que para todos $a_{1}, \ldots, a_{n} \in M^{H}$ existe $b \in M^{H}$ tal que $\left\langle a_{1}, \ldots, a_{n}, b\right\rangle \in P^{M^{H}}$. Então, por definição de $M^{H}, \Sigma_{\omega} \vdash \forall x_{1} \ldots \forall x_{n} \exists y P\left(x_{1}, \ldots, x_{n}, y\right)$, e como $D 2^{\prime \prime}$ tem o Axioma da Escolha,

$$
\Sigma_{\omega} \vdash \exists f \forall x_{1} \ldots \forall x_{n} P\left(x_{1}, \ldots, x_{n}, f\left(x_{1}, \ldots, x_{n}\right)\right) .
$$

Por construção de $V^{\prime}$ e $M^{H}$, existe $p \in V^{\prime}$ tal que $p^{M^{H}} \in F_{n}^{H}$ e

$$
\Sigma_{\omega} \vdash \forall x_{1} \ldots \forall x_{n} P\left(x_{1}, \ldots, x_{n}, p\left(x_{1}, \ldots, x_{n}\right)\right),
$$

e daí a tese segue como em 1.4.2.

Corolário 1.4.6 Se o Axioma Universal da Escolha for adicionado à lista de axiomas de $D 2^{\prime}$ ainda teremos Correção e Completude para L2.

Com a ajuda deste axioma poderemos formar uma teoria de conjuntos onde o axioma da substituição não seja um esquema de axiomas. O axioma da escolha usual de $Z F C$ também pode ser demonstrado com a ajuda deste axioma; na verdade, provaremos no próximo capítulo que ele fornece um princípio de escolha "universal". Agora, um resultado sobre $L 1$ com consequências para os sistemas dedutivos de $L 2$. A prova desta proposição pode ser achada em Kreisel (1968):

Proposição 1.4.7 Seja $\Gamma \subseteq L 1(V)$ tal que prova eliminação de corte para L1, e que para cada natural $k$ tenha uma definição de verdade para todas as fórmulas de complexidade menor ou igual a $k$ e que prova indução para todas as fórmulas da linguagem. Então $\Gamma$ não é finitamente axiomatizável.

Definição 1.4.8 O sistema dedutivo D2+ é o sistema obtido por se adicionar a D2 os axiomas da Compreensão e da Escolha.

Corolário 1.4.9 O sistema dedutivo D2+ não é conservativo sobre D1. 


\section{Capítulo 2}

\section{Segunda Ordem e Teoria de Conjuntos}

\subsection{ZF2 e sua consistência com a Hipótese do Contínuo}

Nesta seção vamos introduzir uma Teoria de Conjuntos de Segunda Ordem, abreviadamente $Z F 2$. $Z F 2$ tem uma propriedade, que chamaremos (seguindo Weston (1976)) de quase-categórica: todos os seus modelos ou são isomorfos entre si ou um é segmento inicial do outro. Além disso, vamos mostrar que todos os modelos de $Z F 2$ são os conjuntos $V_{\kappa}$, para $\kappa$ fortemente inacessível. Estes fatos levaram Kreisel a considerar o estudo de $Z F 2$ de extrema importância, pelo seguinte motivo: em níveis muito inferiores ao do primeiro cardinal inacessível, estão as respostas de muitos problemas importantes, como a da Hipótese do Contínuo $(H C)$. Como todos os modelos de $Z F 2$ são um uma extensão do outro, temos que se $H C$ é satisfeita em algum destes modelos, então $H C$ é satisfeita em todos os modelos (e o mesmo argumento vale para a negação de $H C$ ). Desta forma, $Z F 2$ decide $H C$, em algum sentido (Kreisel (1967)).

Isto poderia levar a pensar que o estudo da Lógica de Segunda Ordem pode ser útil na questão de decidir o valor verdade da $H C$ (se é que existe um tal valor para $H C$ uma discussão sobre o tema é Weston (1974)). No entanto, temos o fato de que o universo de conjuntos subjacente pode ser entendido como um universo de primeira ordem, como normalmente é o caso, e assim sujeito a diferentes interpretações; desta forma, o valor verdade de $H C$ volta a ser um problema em Lógica de Primeira Ordem. Vamos ver isso com mais detalhes ao longo desta seção, e provaremos que a $H C$ não é dedutível em $Z F 2$, mesmo com o sistema dedutivo mais forte que temos, $D 2+$. Atualmente já chegaram até a afirmar que esta redução da $H C$ à validade da Lógica de Segunda Ordem é obviamente de nenhuma ajuda (Van Benthem e Doets (1983)).

Definição 2.1.1 A Teoria de Conjuntos de Segunda Ordem é a teoria dada pelos seguintes axiomas:

$$
\begin{array}{ll}
\forall x \forall y[\forall z(z \in x \leftrightarrow z \in y) \rightarrow x=y] & \text { (extensionalidade) } \\
\forall x \forall y \exists z \forall w(w \in z \leftrightarrow(w=x \vee w=y)) & \text { (pares) } \\
\forall x \exists y \forall z(z \in y \leftrightarrow \exists w(z \in w \wedge w \in x)) & \text { (uniãa) } \\
\forall x \exists y \forall z(z \in y \leftrightarrow \forall w(w \in z \rightarrow w \in x)) & \text { (partes) } \\
\exists x[\exists y(y \in x) \wedge \forall z(z \in x \rightarrow \exists w(w \in x \wedge \forall v(v \in w \leftrightarrow v \in z \vee v=z)))] & \text { (infinidade) } \\
\forall x[\exists y(y \in x) \rightarrow \exists y(y \in x \wedge \forall z(\neg(z \in y \wedge z \in x)))] & \text { (fundaçãa) }
\end{array}
$$

e, por último, o axioma da substituição de segunda ordem:

$$
\forall f \forall x \exists y \forall z[z \in y \leftrightarrow \exists w(w \in x \wedge z=f(w))] .
$$


Chamaremos de ZF2 a conjunção destas sentenças. O axioma da escolha não está presente pois ele é dedutivel destes mais o axioma lógico da escolha 1.4.4, do sistema dedutivo D2+.

Nesta primeira seção nós iremos demonstrar a propriedade de $Z F 2$ ser quase-categórica na semântica padrão. Este teorema foi primeiramente demonstrado em Zermelo (1930), mas a exposição a seguir basea-se na dada em Shepherdson (1952).

Ao longo desta seção, salvo menção contrária, fixamos um modelo $M$ de $Z F 2$ e frequentemente escreveremos $M(x)$ no lugar de $x \in|M|$, para ajudar a evitar confusão entre a pertinência no modelo e na metalinguagem. As letras gregas $\sigma$ e $\tau$ indicarão ordinais e $x \in O N$ será uma abreviação para " $x$ é um ordinal".

A metateoria, ou seja, a teoria de conjuntos que usaremos para modelar a semântica de $Z F 2$ será $Z F C$. $Z F C$ é a teoria de conjuntos de primeira ordem dadas pelos axiomas de primeira ordem de $Z F 2$ mais o axioma da escolha:

$$
\forall u[\forall x \in u(x \neq \emptyset \wedge \forall y \in u(x=y \vee x \cap y=\emptyset)) \rightarrow \exists c \forall x \in u \exists ! y(y \in x \wedge y \in c)]
$$

(ou seja, se $u$ é uma família de conjuntos não vazios dois a dois disjuntos, então existe $v$ contendo um único elemento de cada $x \in u$ ) e o esquema de axiomas da substituição: para cada fórmula $\varphi$ de primeira ordem no vocabulário $\{\in,=\}$, é um axioma:

$$
\forall y[\forall x(x \in y \rightarrow \exists z \varphi) \rightarrow \exists w \forall x(x \in y \rightarrow \exists z(z \in w \wedge \varphi))]
$$

Lembramos que a igualdade de dois objetos em uma estrutura de Henkin não significa, necessariamente, igualdade deles na metateoria (obs. 1.3.12), mas sempre podemos tomar uma outra estrutura onde isso acontece e que satisfaz as mesmas sentenças que a anterior (basta substituir $|M|$ pelo conjunto das classes de equivalência da igualdade). Nesta seção vamos supor que todos os nossos modelos são deste segundo tipo.

Usaremos a convenção de chamar de "conjunto" apenas os objetos de primeira ordem de $M$, sendo que os elementos de $R^{H}$ serão chamados de "classes" e os elementos de $F^{H}$ de "funcionais". É claro que, do ponto de vista da metateoria, todos os objetos em questão são conjuntos.

Por último, frequentemente vamos escrever nossos teoremas de $Z F C$ em algum vocabulário extendendo $\{\in,=\}$; por exemplo, usaremos $P(x)$ para indicar o conjunto dado pelo axioma das partes. No entanto, todos os símbolos que usaremos terão uma definição explícita em $Z F C$, e portanto seu uso é inofensivo (para uma exposição sobre este assunto, ver Hodges (1993)). O mesmo pode acontecer quando trabalharmos em $Z F 2$.

Lema 2.1.2 ZF2 prova o axioma da escolha de $Z F C$.

Prova. Seja $X_{i}, i \in I$, uma família de conjuntos não-vazios. Vamos provar que existe um conjunto $c$ tal que $c \cap X_{i}$ é unitário para todo $i \in I$. Pelo Axioma da Compreensão, existe uma classe $C$ tal que

$$
C=\{\langle x, y\rangle:(x \neq \emptyset \rightarrow y \in x) \wedge(x=\emptyset \rightarrow y=\emptyset)\} .
$$

Então, para todo $x$, existe $y$ tal que $C(x, y)$. Pelo Axioma Universal da Escolha existe um $f$ tal que, para todo $x, C(x, f x)$ e, pelo Axioma da Substituição, $c=f_{*}\left\{X_{i}: i \in I\right\}$ é conjunto e $c \cap X_{i}=\left\{f\left(X_{i}\right)\right\}$ para todo $i \in I$.

Vamos introduzir agora a noção de relativização de uma fórmula, que equivale, para sentenças, à noção de satisfação. 
Definição 2.1.3 (a) Para cada modelo $M$ de $Z F 2$, a relativização (na semântica padrão) de um termo $t$ de $Z F 2$ é dado pela seguinte regra: se $t$ é uma variável $x$, então $t^{M}=x$; se $t$ é um símbolo de constante $c$, então $t^{M}=c^{M}$, se $t$ é $f\left(t_{1}, \ldots, t_{n}\right)$, com $f$ uma variável de função, então $t^{M}=f\left(t_{1}^{M}, \ldots, t_{n}^{M}\right)$, e se $t$ é $p\left(t_{1}, \ldots, t_{n}\right)$, com $p$ um símbolo de função, então $t^{M}=p^{M}\left(t_{1}^{M}, \ldots, t_{n}^{M}\right)$.

(b) Para cada modelo $M$ de $Z F 2$, a relativização (na semântica padrão) de uma fórmula $\varphi$ de $Z F 2$ em $Z F C$ é a fórmula $\varphi^{M}$ de primeira ordem no vocabulário $\{\in,=\}$ obtida por indução na complexidade de $\varphi$ do seguinte modo: variáveis de segunda ordem quaisquer são lidas como variáveis de primeira ordem $e$

(i) $S e \varphi^{M}$ é atômica ${ }^{1}$, então é da forma $P\left(t_{1}, \ldots, t_{n}\right)$; neste caso $\varphi^{M}$ é $\left\langle t_{1}^{M}, \ldots, t_{n}^{M}\right\rangle \in P^{M}$ (onde $P^{M}=P$ se $P$ for uma variável de relação), mas às vezes escreveremos $P^{M}\left(t_{1}^{M}, \ldots, t_{n}^{M}\right)$ (por exemplo, se $x$ é uma variável e $\omega$ um símbolo de constante, então $(x \in \omega)^{M}$ será $x \in \epsilon^{M} \omega^{M}$ ao invés de $\left.\left\langle x, \omega^{M}\right\rangle \in \in^{M}\right)$;

(ii) $(\psi \wedge \chi)^{M}$ é $\psi^{M} \wedge \chi^{M}$, e semelhantemente para $\vee e \rightarrow$;

(iii) $(\neg \psi)^{M} \dot{e} \neg\left(\psi^{M}\right)$;

(iv) se x é uma variável de primeira ordem, então $(\exists x \psi)^{M}$ é $\exists x\left(M x \wedge \psi^{M}\right)$ e $(\forall x \psi)^{M}$ é $\forall x\left(M x \rightarrow \psi^{M}\right)$;

(v) se $X$ é uma variável de relação n-ária, então $(\exists X \psi)^{M}$ é $\exists X\left(X \in P\left(M^{n}\right) \wedge \psi^{M}\right)$ e $(\forall X \psi)^{M}$ é $\forall X\left(X \in P\left(M^{n}\right) \rightarrow \psi^{M}\right)$;

(vi) se $f$ é uma variável de função n-ária, então $(\exists f \psi)^{M}$ é $\exists f\left(F u n\left(f, M^{n}, M\right) \wedge \psi^{M}\right) e$ $(\forall f \psi)^{M}$ é $\forall f\left(F u n\left(f, M^{n}, M\right) \rightarrow \psi^{M}\right)$, onde Fun $(x, y, z)$ é uma fórmula de $Z F$ dizendo que $x$ é função com domínio y e imagem em z.

No restante desta seção $M$ indica um modelo fixado (mas arbitrário) de $Z F 2$.

Lema 2.1.4 A relação $\in^{M}$ é bem-fundada em $M$.

Prova. Suponha que exista uma sequencia $x_{i}, i \in \omega$, tal que $x_{i+1} \in \in^{M} x_{i}$ para todo $i$. Notando que $x \in \in^{M} y \rightarrow M x \wedge M y$ (já que $\in^{M} \subseteq M^{2}$ ), a relativização do axioma da substituição fornece, se $f$ é uma função em $M$,

$$
\forall x\left(M x \rightarrow \exists y \forall z\left(z \in^{M} y \leftrightarrow \exists w\left(w \in^{M} x \wedge z=f w\right)\right)\right) .
$$

Seja $f: M \rightarrow M$ definida assim: $f(y)=x_{i}$, se $y=i^{M}$ e $f(y)=0^{M}$, caso contrário. Pela relativização do axioma da substituição, tomando $x=\omega^{M}$, existe $y \in M$ tal que $z \in^{M} y \leftrightarrow \exists w\left(w \in^{M} \omega^{M} \wedge z=f w\right)$. Mas tal conjunto não pode existir, pela relativização do axioma da regularidade.

Proposição 2.1.5 (a) Defina o seguinte símbolo de função $H$ (em ZFC):

$$
H(\sigma)=\left\{x \in M: \forall y\left[y \in^{M} x \rightarrow \exists \tau(\tau<\sigma \wedge y \in H \tau)\right]\right\} .
$$

Então $M x \leftrightarrow \exists \sigma(x \in H \sigma)$.

(b) Considere $H$-rank $(x)$, o menor ordinal $\sigma$ que $x \in H \sigma$. Defina transfinitamente em $H$-rank o simbolo de função F tal que

$$
F(y)=\left\{F(x): M x \wedge x \in^{M} y\right\}
$$

(os detalhes de como funçôes podem ser definidas transfinitamente desse modo podem ser encontrados em Shoenfield (1967)). Neste caso, definindo $\left|M^{\prime}\right|=F_{*}(M)$ e $M^{\prime}=\left\langle\left|M^{\prime}\right|, \in^{M^{\prime}}\right\rangle$ tal que $x \in^{M^{\prime}} y \leftrightarrow x \in y$, temos que se $M^{\prime} x \wedge z \in x$ então $M^{\prime} z$. Além disso, $F: M \cong M^{\prime}$.

\footnotetext{
${ }^{1}$ onde estamos considerando $t=s$ atômica, para $t, s$ termos, segundo a obs. 1.3.12.
} 
Prova. (a) Por definição de $H, x \in H \sigma \rightarrow M x$; para ver a implicação contrária, suponha que $\exists y(y \in M \wedge \neg \exists \sigma(y \in H \sigma))$. Como $\in^{M}$ é bem-fundada em $M$ e o subconjunto $X=\{x$ : $\neg \exists \alpha(x \in H \alpha)\}$ de $M$ é não-vazio, temos

$$
\exists y\left(y \in X \wedge \forall z \neg\left(z \in^{M} y \wedge z \in X\right)\right) ;
$$

$\operatorname{logo} \exists y\left(y \in X \wedge \forall z\left(z \in^{M} y \rightarrow \exists \sigma(z \in H \sigma)\right)\right.$. Considere o conjunto $S=\left\{\alpha: \exists z\left(z \in \in^{M}\right.\right.$ $y \wedge \alpha=H-\operatorname{rank}(z))\}$; então $\rho=\bigcup S \cup\{\bigcup S\}$ é um ordinal maior que todo elemento de $S$ e, como todos os $M$-membros de $y$ estão em $\bigcup H \alpha$, eles tem $H$-rank definido; logo

$$
z \in^{M} y \rightarrow H-\operatorname{rank}(z)<\rho,
$$

isto é, para todo $z M$-membro de $y, \exists \beta(\beta<\rho \wedge z \in H \beta)$. Assim, como $y \in M$ e todo $M$-elemento de $y$ está em algum $H \beta \operatorname{com} \beta<\rho$, temos $y \in H \rho$, um absurdo.

(b) Suponha que $M^{\prime} x \wedge z \in x$. Então $\exists y(M y \wedge x=F(y))$. Logo $z \in F(y)$, e assim $z=F(w)$ para algum $w$ tal que $M w$, donde $M^{\prime} z$.

Vamos mostrar que $F$ é o isomorfismo procurado. Só é necessário mostrar que

$$
\begin{gathered}
M x \wedge M y \rightarrow\left(x \in^{M} y \leftrightarrow F x \in F y\right) \text { (isto é, que } \in \text { é preservada), e que } \\
M x \wedge M y \rightarrow(x=y \leftrightarrow F x=F y) \text { (isto é, que } F \text { é injetora). }
\end{gathered}
$$

Suponha então que $M x$ e $M y$; se $x \in^{M} y$, então, por definição de $F$,

$$
F(y)=\left\{F(x): M x \wedge x \in^{M} y\right\}
$$

donde $F(x) \in F(y)$. Se $F(x) \in F(y)$, segue que $x \in^{M} y$, novamente por definição. Suponha agora que $F(x)=F(y)$; vamos usar indução em $H$-rank. Pela relativização do axioma da extensionalidade em $M$,

$$
\forall z\left(M z \rightarrow\left(z \in^{M} x \leftrightarrow z \in^{M} y\right)\right) \rightarrow x=y .
$$

Seja então $z \in^{M} x$; então $F(z) \in F(x)=F(y)$; logo $F(z)=F(w)$ para algum $w \in^{M} y$. Por hipótese de indução $z=w$, donde $z \in^{M} y$. Análogamente $z \in^{M} y \rightarrow z \in^{M} x$ e assim $x=y$.

Definição 2.1.6 (a) Um modelo $M$ de $Z F 2$ é um $\in$-modelo se satisfaz $x \in \in^{M} y \leftrightarrow x \in y$.

(b) Um modelo $M$ é dito transitivo se $M x \wedge y \in x \rightarrow M y$.

(c) Uma fórmula $\varphi$ é absoluta para $M$ se $\varphi \leftrightarrow \varphi^{M}$. Dizemos simplesmente que $\varphi$ é absoluta se $\varphi$ for absoluta para um $\in-$ modelo transitivo qualquer. Dizemos também que um conjunto a é absoluto se existe $\varphi$ tal que $x \in a \leftrightarrow \varphi(x)$ e $\varphi$ é absoluta.

Lema 2.1.7 Fórmulas livres de quantificadores são absolutas, e todas as relaçôes, símbolos de funções e conjuntos a seguir são definidos por fórmulas absolutas:

$\begin{array}{llll}x \subseteq y, & x=y, & \emptyset, & \{x, y\}, \\ \langle x, y\rangle, & \left\langle x_{1}, \ldots, x_{n}\right\rangle, & \bigcup_{x} x, & x^{+}, \\ x \cap y, & x-y, & \bigcap x(\operatorname{com} \cap \emptyset=\emptyset), & A \times B, \\ \text { "R é relação", } & \operatorname{Im}(R), & \operatorname{Func}(f), & \operatorname{dom}(f), \\ \text { "x é a primeira coordenada "x é transitivo", } & \operatorname{Ord}(x), & R \text { é boa ordem em } A, \\ \text { de uma n-upla, n fixado" } & \omega, & \operatorname{os~} n \in \omega, & \operatorname{rank}(x),\end{array}$

- $F(x)$ é o menor ordinal $\sigma$ tal que $\varphi(\sigma, x) \operatorname{com} \varphi$ absouluta, 
- F é um símbolo de função definido por indução transfinita em termos de um símbolo de função absoluto.

Prova. Será omitida; pode ser encontrada em Shoenfield (1967).

Graças à Proposição 2.1.5, podemos assumir agora que $M$ é um $\in$-modelo transitivo. Além disso, como $\operatorname{Ord}(x)$ é absoluta, $O N^{M}$, a classe dos ordinais em $M$, é um ordinal (na metateoria).

Definição 2.1.8 (a) Um cardinal $\sigma$ é dito inacessível se:

(i) $\omega<\sigma$,

(ii) para todo cardinal $\tau, \tau<\sigma \rightarrow 2^{\tau}<\sigma e$

(iii) $\forall x \subseteq \sigma(\operatorname{Card}(x)<\sigma \rightarrow \bigcup x<\sigma)$ (onde Card é o simbolo de função que associa a cada $x$ seu cardinal).

(b) O Axioma da Inacessibilidade é a sentença $\exists x \operatorname{Inac}(x)$, que afirma a existência de um cardinal inacessivel. ZFI é a teoria obtida de ZFC por se adicionar o Axioma da Inacessibilidade.

Observação 2.1.9 Sobre cardinais inacessíveis. O Axioma da Inacessibilidade é realmente um axioma, isto é, não pode ser demonstrado em ZFC (no caso de ZFC ser consistente, é claro). Na verdade, ele é de um tipo de axioma cuja consistência com ZFC não pode ser provada em $Z F C+C o n(Z F C)$ (algo que pode ser feito para $H C$ ). Isto se deve pelo fato de $Z F I$ provar $C o n(Z F C)$ : se $Z F C+C o n(Z F C)$ provasse $C o n(Z F I)$, então $Z F I$ provaria Con $(Z F I)$ (Shoenfield (1967)).

Teorema 2.1.10 $O N^{M}$ é um ordinal inacessível.

Prova. Veja primeiro que $\omega<O N^{M}$ segue do fato que $\omega$ é absoluto (lema 2.1.7). Vamos inicialmente mostrar que se $\tau$ é um cardinal, então

$$
\tau<O N^{M} \rightarrow 2^{\tau}<O N^{M} .
$$

Para ver isto, note primeiro que o conjunto das partes de um $x$ arbitrário é absoluto para $M$; de fato, se $M x$,

$$
P^{M}(x)=\{y: y \subseteq x\}^{M}=\left\{y: M y \wedge y \subseteq^{M} x\right\}=\{y: M y \wedge y \subseteq x\}=P(x) \cap|M| .
$$

Mas se $x \in M$ e $X \subseteq x$, então $X \subseteq M$, pois $M$ é transitivo. Agora, o princípio dos subconjuntos de segunda ordem é a fórmula

$$
\forall X \forall x \exists y \forall z(z \in y \leftrightarrow z \in x \wedge X(x)),
$$

e pode-se demonstrá-lo em $Z F 2$ do mesmo modo que o mesmo princípio de primeira ordem é demonstrado em $Z F C$. Sua relativização fica, se $X \in P(M)$ e $x \in M$,

$$
\exists y(M y \wedge \forall z(z \in y \leftrightarrow z \in x \wedge z \in X)) .
$$

Logo $y=x \cap X$; como $X \subseteq x$, temos $y=X \in M$. Segue que partes é absoluto; chamaremos um modelo com esta propriedade de supertransitivo.

Agora $2^{\sigma}=\operatorname{Card}(P(\sigma))$ é absoluto para modelos supertransitivos (Shoenfield (1967)). Logo $2^{\tau} \in M$, e assim $2^{\tau}<O N^{M}$.

Vamos mostrar agora que

$$
\forall x\left(x \subseteq O N^{M} \wedge \operatorname{Card}(x)<O N^{M} \rightarrow \bigcup x<O N^{M}\right) .
$$


Como $\operatorname{Card}(x)<O N^{M}$, temos $\operatorname{Card}(x) \in M$ e, como existe uma bijeção $f: \operatorname{Card}(x) \rightarrow x$, o axioma da substituição relativizado garante que $x \in M$. Como tomar a união de um conjunto é uma operação absoluta (lema 2.1.7), e como $x$ é um conjunto de ordinais, $\bigcup x$ é um ordinal que está em $M$. Assim $\bigcup x \in O N^{M}$.

Teorema 2.1.11 $M=V_{\rho}$, onde $\rho=O N^{M}$ e $V$ é o símbolo de função (de ZFC) tal que $V_{0}=0, V_{\alpha+1}=P\left(V_{\alpha}\right)$ e $V_{\lambda}=\bigcup_{\alpha<\lambda} V_{\alpha}$, se $\lambda$ for limite.

Prova. Primeiro, note que $\rho$ é limite: de fato, se $\rho=\sigma+1$, então $\sigma \in \rho$; mas como $\rho=O N^{M}=O N \cap M$, temos $\sigma \in M$, donde $\sigma \cup\{\sigma\} \in M$ (pois sucessor é absoluto) e assim $O N^{M} \in M$, um absurdo.

Agora, é claro que como $M$ é supertransitivo, se $\alpha \in M$, então

$$
V_{\alpha}^{M}=V_{\alpha}
$$

Vamos mostrar que $M=\bigcup_{\alpha<\rho} V_{\alpha}$. Como $V_{\alpha}^{M} \subseteq M$, só falta mostrar que $\neg \exists y(y \in$ $\left.M-\bigcup_{\alpha<\rho} V_{\alpha}\right)$; suponha o contrário. Então, como $\in$ é bem-fundada em $M$, existe $y \in M$ tal que

$$
S=\{\alpha: \exists z(z \in y \wedge \operatorname{rank}(z)=\alpha)\} \neq \emptyset ;
$$

e $\operatorname{assim} \beta=\bigcup S \cup\{\bigcup S\}$ é maior que o rank de todo $z \in y$, donde $y \in V_{\beta}$, um absurdo.

Isto completa a prova da quase-categoricidade de $Z F 2$. Agora, vamos mostrar que $H C$ é consistente com $Z F 2$ (em relação à $D 2+$ ).

Primeiro, vamos mostrar que se assumirmos na metateoria o axioma da construtibilidade (cuja definição pode ser encontrada em Shoenfield (1967)), então a relativização deste axioma a um modelo de $Z F 2$ é demonstrável; disto seguirá que podemos demonstrar a interpretação da Hipótese Generalizada do Contínuo, e assim $H C$ será consistente com $Z F 2$ (se, é claro, $Z F C$ for consistente).

Teorema 2.1.12 Suponha que na metateoria vale o axioma da construtibilidade, isto é, que $\forall x L(x)$. Então, se $M$ é um modelo de $Z F 2$, vale que

$$
L^{M}(x) \leftrightarrow M(x) .
$$

Prova. Podemos supor que $M=V_{\rho}$, com $\rho$ inacessível e que $M$ é $\in-$ modelo. Por definição, temos

$$
L x \leftrightarrow \exists y(\operatorname{Ord}(y) \wedge x=C(y))
$$

(onde $C$ é o símbolo de função usado na definição do axioma da construtibilidade em Shoenfield (1967)). Agora, $C$ é absoluto para $\in$-modelos transitivos. Logo

$$
L^{M}(x) \leftrightarrow \exists y(M y \wedge \operatorname{Ord}(y) \wedge x=C(y)) .
$$

Veja que como $C$ é absoluto, se $\tau<\rho$, então $\tau \in M$ e $C^{M}(\tau)=C(\tau) \in M$, e portanto nós temos $C_{*}(\rho) \subseteq M$, donde o que queremos mostrar é que ${ }^{2}$

$$
M \subseteq C_{*}(\rho) .
$$

\footnotetext{
${ }^{2}$ Vamos seguir a nossa notação, usando $C_{*}(\rho)$ para denotar a imagem do símbolo de função $C$ pelo conjunto $\rho$, mas chamamos à atenção para o fato de que em Shoenfield (1967) a notação usada é $C^{*}(\rho)$.
} 
Note porém que é suficiente mostrar que cada $V_{\alpha}$ para $\alpha<\rho$ é igual a um $C(\gamma)$, para algum $\gamma<\rho$ : de fato, se $x \in C(\gamma)$ então $H-\operatorname{rank}(x)<\gamma$, isto é, $H-\operatorname{rank}(x) \in \gamma$, donde $H-\operatorname{rank}(x) \in V_{\rho}$, e assim $x=C(H-\operatorname{rank}(x)) \in V_{\rho}$.

Vamos então provar por indução transfinita em $\alpha$ que

$$
\alpha<\rho \rightarrow \exists \gamma\left(\gamma<\rho \wedge V_{\alpha}=C(\gamma)\right) .
$$

Se $\alpha=0$, então $V_{0}=0=C(0)$. Se $\alpha=\beta+1$, então $V_{\alpha}=P\left(V_{\beta}\right)$; por hipótese de indução existe um $\gamma$ menor que $\rho$ com $V_{\beta}=C(\gamma)$. Como $\rho$ é inacessível, existe um ordinal $\mu$ com $\gamma<\aleph_{\mu}<\rho$ de tal forma que

$$
\begin{gathered}
V_{\beta}=C(\gamma) \subseteq C_{*}\left(\aleph_{\mu}\right), \\
\text { e assim } V_{\alpha}=P\left(V_{\beta}\right) \subseteq P\left(C_{*}\left(\aleph_{\mu}\right)\right) .
\end{gathered}
$$

Agora em Shoenfield (1967) mostra-se que

$$
P\left(C_{*}\left(\aleph_{\mu}\right)\right) \subseteq C_{*}\left(\aleph_{\mu+1}\right),
$$

donde segue que $V_{\alpha} \in P\left(C_{*}\left(\aleph_{\mu+1}\right)\right) \subseteq C_{*}\left(\aleph_{\mu+2}\right)$. Como a inacessiblidade de $\rho$ implica $\aleph_{\mu}<$ $\rho \rightarrow \aleph_{\mu+2}<\rho$, segue a conclusão desejada. Agora, se $\alpha$ for limite, sejam $V_{\alpha}^{\prime}=\left\{V_{\beta}: \beta<\alpha\right\}$ e $(H-\operatorname{rank})_{*}(x)=\{H-\operatorname{rank}(y): y \in x\}$. Por hipótese de indução,

$$
(H-\operatorname{rank})_{*}\left(V_{\alpha}^{\prime}\right) \subseteq \rho,
$$

e além disso

$$
\operatorname{Card}\left((H-\operatorname{rank})_{*}\left(V_{\alpha}^{\prime}\right)\right) \leq \operatorname{Card}\left(V_{\alpha}^{\prime}\right) \leq \operatorname{Card}(\alpha) \leq \alpha<\rho .
$$

A inacessibilidade de $\rho$ implica que $\bigcup(H-r a n k)_{*}\left(V_{\alpha}^{\prime}\right)<\rho$ e que

$$
\exists \mu\left(\aleph_{\mu}<\rho \wedge \forall \beta\left(\beta<\alpha \rightarrow H-\operatorname{rank}\left(V_{\beta}\right)<\aleph_{\mu}\right)\right) .
$$

Isto então fornece

$$
\beta<\alpha \wedge x \in V_{\beta} \rightarrow H-\operatorname{rank}(x)<\aleph_{\mu},
$$

e assim

$$
x \in V_{\alpha} \leftrightarrow \exists \beta\left(\beta<\alpha \wedge \alpha \in V_{\beta}\right) \rightarrow H-\operatorname{rank}(x)<\aleph_{\mu} .
$$

Portanto $V_{\alpha} \subseteq C_{*}\left(\aleph_{\mu}\right)$. Segue então que

$$
V_{\alpha} \in P\left(C_{*}\left(\aleph_{\mu}\right)\right) \subseteq C_{*}\left(\aleph_{\mu+1}\right),
$$

isto é,

$$
\exists \sigma\left(\sigma<\aleph_{\mu+1} \wedge V_{\alpha}=C(\sigma)\right) .
$$

Como $\aleph_{\mu}<\rho \rightarrow \aleph_{\mu+1}<\rho$, isto completa a prova.

Corolário 2.1.13 (a) Se ZFI é consistente, então ZF2 mais o Axioma da Construtibilidade é consistente.

(b) Se ZFI é consistente, então ZF2 mais a Hipótese Generalizada do Contínuo é consistente. 


\subsection{Forcing em ZF2}

Agora vamos começar a falar de forcing. Nosso primeiro objetivo será estabelecer a independência da Hipótese do Contínuo. No entanto, devido ao nosso método de forcing, será necessário supor a existência de um $\in-$ modelo transitivo de Henkin de $Z F 2$. A exposição a seguir basea-se na dada em Chuaqui (1972), mas com algumas modificações, de tal forma que inicialmente faremos forcing para conjuntos (já que Chuaqui (1972) o faz para classes); no final, isso possibilitará provar a independência de várias sentenças para $Z F 2$ (no sistema $D 2+)$.

Antes de começarmos, algumas observações. Em toda esta seção, toda referência a modelos será a modelos de Henkin e faremos muitos abusos de linguagem usuais (como por exemplo $\exists x \in M \varphi$ para indicar $\exists x(x \in M \wedge \varphi)$ para facilitar a compreensão do texto. Iremos também usar a seguinte notação pouco usual de $n$-uplas: uma única letra latina, maiúscula ou minúscula, representará uma $n$-upla, isto é, teremos algo como $\varphi(a)$ abreviando $\varphi\left(a_{1}, \ldots, a_{n}\right)$. O usual é escrever $\bar{a}$ para indicar a $n$-upla; decidimos, no entanto, fazer deste modo pois tratamentos antigos de forcing usavam $\bar{a}$ para indicar a interpretação em $M[G]$ de um nome $a$. Não querendo confundir o leitor (e nem a nós mesmos) fizemos esta convenção, de tal forma que $\bar{a}$ não aparecerá em nenhum momento deste capítulo. A aridade da $n$-upla será qualquer aridade necessária para o texto fazer sentido. Desta forma, $a$ pode até mesmo ser um único elemento.

Alguma motivação. ${ }^{3}$ Suponha que $M$ seja um $\in$-modelo transitivo arbitrário de $Z F 2$. Vamos tentar criar um outro modelo de $Z F 2$ que, em algum sentido, seja uma extensão de $M$, mas que também contenha outros conjuntos que $M$ não tem. Desta forma, esta extensão satisfará fórmulas que $M$ não precisava satisfazer e, por ser um modelo de $Z F 2$, vai seguir que estas fórmulas são consistentes com $Z F 2$.

Vamos começar com um conjunto $C \in M$, cujos elementos chamaremos de condições. A ideia é formar um conjunto a partir das das condições em $C$, um $G \subseteq C$, mas de tal forma que $G \notin M$, isto é, $G \in P(C)-P^{M}(C)$. Mas vamos, ainda, querer algo mais sobre $G$ : $G$ deverá ter apenas as propriedades absolutamente necessárias para o que queremos a seu respeito. Desta forma, teremos algum controle sobre a formação de $G$. Como $G$ deve ser tão arbitrário, vamos chamá-lo de um genérico, ou mais precisamente, um $C$-genérico sobre $M$.

Para obtermos tal $G$, vamos proceder assim: as propriedades que queremos que $G$ satisfaça serão determinadas pelas condições em $C$. De certa forma, já é isso que acontece, dado que $G$ é um subconjunto de $C$. Mas o que queremos dizer é que queremos definir uma relação entre elementos de $C$ e fórmulas, que chamaremos de forcing, de tal forma que $p \Vdash \varphi$ signifique que $\varphi$ é uma sentença que é garantida ser válida por $p$ se $p \in G$, isto é, para cada sentença $\varphi, \varphi$ será válida se alguma condição $p$ em $G$ "forçar" $\varphi$. Desta forma, as condições em $G$ seriam, em algum sentido, protótipos de sentenças consistentes entre si.

Agora, se quisermos pensar que $C$ é um protótipo de um conjunto de sentenças, do qual $G$ é um conjunto consistente, somos então levados a pensar que talvez seja bom que $C$ seja uma álgebra de Boole; mas não vamos precisar ir tão longe. Como queremos apenas que $G$ seja consistente, bastará que $G$ seja algo como um filtro ${ }^{4}$ (ou um ideal, dependendo da forma que se toma a ordem). Assim, vamos mudar um pouco ainda a intuição sobre as condições em $C$ : elas não serão protótipos de sentenças, mas protótipos de conjuntos de sentenças, de

\footnotetext{
${ }^{3}$ A explanação seguinte não é a forma como as ideias surgiram; são mais alguns pensamentos que ocorreram ao autor conforme ele tentava entender certas definições. Uma exposição das ideias originais pode ser encontrada em Cohen (2008).

${ }^{4}$ Um filtro $G$ numa ordem parcial $C$ é um $G \subseteq C$ não vazio tal que se $p \in G$ e $p \leq q$, então $q \in G$ e, se $p, q \in G$, então existe $r \in G$ tal que $r \leq p$ e $r \leq q$.
} 
tal forma que se $p \leq q$, então é porque $p$ "decide" ao menos tudo o que $q$ conseguia decidir; em algum sentido, há o mesmo tanto de informação ou mais sobre $G$ em $p$ do que em $q$. Desta forma, vamos pensar mesmo em $G$ como um filtro, e $C$ como uma ordem parcial. Também, podemos pensar que cada elemento de $G$ aproxima um pouco um determinado conjunto e, se $q<p$ e $q \in G$, então $q$ aproxima este conjunto ainda mais. O conjunto que aproximamos pelas condições seria então, na verdade, $\bigcup G$. Por exemplo, se queremos construir uma função injetora $\bigcup G: 2^{\omega} \rightarrow \omega_{1}$, deveríamos tomar como condições funções injetoras parciais de $2^{\omega}$ em $\omega_{1}$.

Será útil, técnicamente, que $C$ tenha um máximo, digamos 1 , de tal forma que as fórmulas forçadas por 1 são aquelas que precisam ser válidas para todo $G$ (por exemplo, $\varphi \vee \neg \varphi$ para qualquer $\varphi$ ). Vamos por algumas destas ideias numa definição.

Definição 2.2.1 Uma noção de forcing em $M$ é uma ordem parcial $\langle C, \leq\rangle \in M$ com maior elemento, que indicamos por 1 . Os elementos de $C$ são chamados de condições e, se $p \leq q$, dizemos que $p$ estende $q$. Se dadas as condições $p$ e q existir uma condição $r$ tal que $r \leq p$ e $r \leq q$, dizemos que $p$ e q são compativeis; se existir a maior das condições que estendem $p$ e $q$, ela se escreve $p \wedge q$.

A relação de forcing precisará satisfazer algumas propriedades; por exemplo, para cada $\varphi$ deverá existir $p \in G$ tal que $p \Vdash \varphi$ ou $p \Vdash \neg \varphi$, mas não os dois. Será um problema que consideraremos futuramente como definir $p \Vdash \varphi$ para fórmulas atômicas, mas para fórmulas não-atômicas será útil pensar na definição agora. Podemos, é claro, definir por indução na complexidade das fórmulas: $p \Vdash \psi \wedge \chi$ se $p \Vdash \psi$ e $p \Vdash \chi$ (não deve existir nada mais razoável do que isso); $p \Vdash \forall x \psi$ se, para todo $x, p \Vdash \psi(x)$ (é uma questão a ser considerada onde $x$ toma valores, mas fora isto, também é uma definição bem natural). Agora, a questão de se $p \Vdash \neg \varphi$ merece especial atenção: não é do nosso interesse definir, por exemplo, $p \Vdash \neg \varphi$ como $\neg p \Vdash \varphi$; pois neste caso $p$ forçaria, para toda $\varphi, \varphi$ ou $\neg \varphi$, e desta forma uma única condição $p$ decidiria tudo a respeito de $G$, e aí seria de se esperar que $G \in M$ (a não ser que, talvez, $\Vdash$ não seja definível em $M$ ). Mas há uma outra maneira de definir $p \Vdash \neg \varphi$ mais de acordo com nossa ideia de que as condições são aproximações: se $p$ é uma aproximação, é razoável de se esperar que existam sentenças que $p$ não "saiba decidir" se são válidas ou não, isto é, não há problema em que exista $\varphi$ tal que $\neg(p \Vdash \varphi)$ e $\neg(p \Vdash \neg \varphi)$. Mas então, quando deveríamos ter $p \Vdash \neg \varphi$ ? Pensando em $G$ como um conjunto de condições consistentes, deveríamos ter um $p \in G$ tal que $p \Vdash \neg \varphi$ somente se for o caso em que não há $q \in G$ tal que $q \Vdash \varphi$. Como $G$ é um filtro, basta exigirmos isto para os $q$ que estão abaixo de $p$; assim, vamos definir $p \Vdash \neg \varphi \Leftrightarrow \forall q \leq p \neg(q \Vdash \varphi)$.

Com estas definições em mãos, passaremos a ver agora que propriedade, ou propriedades, $G$ precisará satisfazer para, por assim dizer, satisfazer o princípio do terceiro excluído. Queremos que, para cada $\varphi$, exista $p \in G$ tal que $p \Vdash \varphi \vee p \Vdash \neg \varphi$, e somente um dos dois. Mas, pela definição de $p \Vdash \neg \varphi$, e por $G$ ser um filtro, não poderá valer ambos. Então, em outras palavras, o que queremos é que, se $D_{\varphi}=\{p: p \Vdash \varphi \vee p \Vdash \neg \varphi\}$, então $G \cap D_{\varphi} \neq \emptyset$. Agora, pensando ainda em $G$ como um protótipo de uma coleção de conjuntos de sentenças consistentes, podemos pensar também em como caracterizar, de modo abstrato, olhando apenas para a ordem parcial, os conjuntos $D_{\varphi}$. Assim, perguntamos: quais propriedades um $D$ contido numa ordem parcial precisa satisfazer para que $D$ seja um $D_{\varphi}$, para alguma $\varphi$ ? A propriedade que chamaremos a atenção que $D_{\varphi}$ tem é esta: seja $p \in G$. Então, existe alguma $\varphi$ tal que $\neg(p \Vdash \varphi) \wedge \neg(p \Vdash \neg \varphi)$; mas deve existir também uma $q \leq p$ tal que $q \Vdash \varphi \vee q \Vdash \neg \varphi$ que esteja em $G$, se é que podemos decidir tudo a respeito de $G$ com as condições em $C$. Em outras palavras, para todo $p \in G$, existe $q \leq p$ tal que $q \in G \cap D_{\varphi}$, para toda $\varphi$. Pondo de um modo mais geral, já que um genérico deve poder ser construído a partir de qualquer $p$ 
(esperamos), temos que $\forall p \exists q \leq p\left(q \in D_{\varphi}\right)$. Chamaremos um conjunto com esta propriedade de um conjunto denso.

Assim, observe então que se exigirmos que $G \cap D \neq \emptyset$ para todo denso $D$, então temos uma condição que não temos motivos para crer que seja necessária, mas que é suficiente para que $G$ possa ter "informação suficiente" para forçar tudo o que for preciso. Mas, na verdade, ela será decisiva para que possamos obter $G \notin M$; vejamos como. Primeiro, note que se $\Vdash$ for definível em $M$, então os $D_{\varphi}$ serão conjuntos em $M$, e consequentemente a condição sobre os densos passa a ser que $G \cap D \neq \emptyset$ para todo $\underline{D \in M}$ denso. Observe agora uma espécie de simetria conceitual entre um filtro $G$ e um denso $D$. Podemos pensar que um filtro é algo que "sobe", enquanto que um denso é algo que pode "descer" arbitrariamente. Assim, o complemento de $G$ em $C$ pode muito bem ser um denso, por ser justamente o "contrário" de um filtro. Agora $G \in M \leftrightarrow C-G \in M$; assim, para que $G \notin M$, basta que $D=G-C \notin M$. Vamos então tentar fazer com que $D$ seja um denso em $M$; se isto acontecer, não poderá valer que $G \cap D \neq \emptyset$, e aí $D \notin M$, donde $G \notin M$. Bom, dado $p \in C$, se $p \notin G$, então $p \in D$. Agora, se $p \in G$, o que é necessário para que exista $q \leq p$ tal que $q \in D$ ? Pensando intuitivamente, sabemos que deve existir alguma $\varphi$ tal que $\neg(p \Vdash \varphi) \wedge \neg(p \Vdash \neg \varphi)$, e que deve existir $q \leq p$ tal que $q \Vdash \varphi \vee q \Vdash \neg \varphi$. Mas, se também existir $r \leq p$ forçando o contrário do que $q$ forçar, então ou $r$ ou $q$ não poderá estar em $G$, e assim um deles estará em $D$. Assim, a condição que falta é

$$
\text { (*) } \forall p \in C \exists q, r \leq p(q \text { é incompatível com } r) .
$$

Neste caso, $G \notin M$. Vamos então agora definir um genérico, e provar que existe ao menos um (aqui será necessário fortalecer a hipótese sobre $M: M$ precisará ser enumerável). Uma última observação sobre a condição (*): ela só é necessária para provar que $G \notin M$; assim, não iremos exigi-la em nossas considerações até que seja estritamente necessário.

Definição 2.2.2 Seja $C$ uma noção de forcing e $D \subseteq C$; então

(a) $D$ é $C$-denso se $\forall p(p \in C \rightarrow \exists q(q \in D \wedge q \leq p))$.

(b) $D$ é $C$-denso abaixo de $p$ se $\forall q(q \leq p \rightarrow \exists r(r \in D \wedge r \leq q))$.

Definição 2.2.3 Seja $C$ uma noção de forcing em $M$. Um $C$-genérico sobre $M$ é um $G \subseteq C$ tal que:
(a) $1 \in G$;
(b) $p \leq q$ e $p \in G$ implicam $q \in G$;
(c) Se $p, q \in G$, então existe $r \in G$ tal que $r \leq p, q$ e
(d) Para todos os $D \in M$ que são $C$-densos, $D \cap G \neq \emptyset$.

Lema 2.2.4 Se $M$ é um $\in$-modelo enumerável de $Z F 2, C$ uma noção de forcing em $M e$ $p \in C$, então existe um $G$ que é $C$-genérico sobre $M$ tal que $p \in G$.

Prova. Seja $D_{n}$ uma enumeração de todos os subconjuntos densos de $C$ que estão em $M$. Escolha indutivamente uma sequência $q_{n}, n \in \omega$ tal que $p=q_{0} \geq q_{1} \geq \ldots$ e $q_{n+1} \in D_{n+1}$; tome então $G=\left\{q: q \geq q_{n} \wedge n \in \omega\right\}$.

Agora queremos definir um modelo $M[G]$, formado a partir do $M$ que contém o conjunto $C$ de condições e que contenha o genérico $G$ (que já sabemos existir se $M$ for enumerável). Nossa ideia é simples: vamos começar definindo uma nova relação de pertinência, que chamaremos de $\epsilon^{G}$, que pode ser pensado como " $a$ pertence a $b$ segundo $G$ ", isto é, em $G$ deverá haver a informação de que " $a$ pertence a $b$ ". Uma forma simples de definir algo assim é por 


$$
a \in^{G} b \Leftrightarrow \exists p \in G(\langle a, p\rangle \in b) .
$$

Veja que sob esta definição podemos fazer algumas operações comuns de formação de conjuntos. Por exemplo, dados os conjuntos $a$ e $b$, podemos formar o par não-ordenado $c$ de $a$ e $b$ "segundo $G$ " assim: $c=\{\langle a, 1\rangle,\langle b, 1\rangle\}$; desta forma, $x \in{ }^{G} c \leftrightarrow x=a \vee x=b$. Na verdade, a partir deste exemplo, parece que qualquer conjunto criado a partir dos axiomas de $Z F 2$ parece poder ser "copiado" de um jeito tal que o novo conjunto tem as mesmas propriedades que seu "original" tinha. Além disso, observe que o conjunto $\operatorname{diag}(C \times C)=\{\langle x, x\rangle: x \in C\}$ é tal que $x \in \in^{G} \operatorname{diag}(C \times C) \leftrightarrow x \in G$. Assim, é possível formar uma espécie de representante de $G$ a partir desta relação $\in^{G}$ e $M$. Também não esperamos poder formar nenhum outro conjunto além de $G$ e dos que estão em $M$ (e dos que podem ser criados em cima destes), já que as operações de $Z F 2$ parecem poder ser aplicadas somente em $M$ e em $G$. Isso parece promissor: $M[G]$ será, como vamos provar, o menor modelo que estende $M$ e que conterá $G$. Mas antes de nos empolgarmos demais com $\epsilon^{G}$, vejamos que há um certo problema com a igualdade: por conta do axioma da extensionalidade, a relação $\epsilon^{G}$ induz uma relação de igualdade, $={ }^{G}$ :

$$
x={ }^{G} y \Leftrightarrow \forall z\left(z \in^{G} x \leftrightarrow z \in^{G} y\right) .
$$

Esta igualdade satisfaz $x={ }^{G} y \wedge z \in^{G} x \rightarrow z \in^{G} y$, mas não tem motivo para satisfazer

$$
x={ }^{G} y \wedge x \in^{G} z \rightarrow y \in^{G} z .
$$

Podemos tentar resolver isto tentando tomar classes de equivalência, algo como

$$
b^{G}=\bigcup_{x \in{ }^{G} b}\left\{y: y={ }^{G} x\right\} .
$$

Mas, em Teoria de Conjuntos, os elementos do universo são construídos a partir de elementos do próprio universo, isto é, temos que $b=\{x: x \in b\}$; assim, se $b^{G}$ deverá ser um conjunto em algum universo, seria de se esperar que ele seja construído a partir de outros conjuntos deste universo, isto é, somos tentados a definir

$$
b^{G}=\bigcup_{x \in G_{b}}\left\{y^{G}: y={ }^{G} x\right\},
$$

e como $y={ }^{G} x \rightarrow y^{G}=x^{G}$, temos

$$
b^{G}=\left\{x^{G}: x \in^{G} b\right\}
$$

Desta forma, observe que $a^{G} \in b^{G} \Leftrightarrow a \in^{G} b$, isto é, a informação que " $a$ pertence a $b$ segundo $G^{\prime \prime}$ é preservada na relação comum de pertinência nos conjuntos $x^{G}$. Definiremos então o domínio dos elementos de primeira ordem de nosso modelo $M[G] \operatorname{como} M[G]=\left\{x^{G}\right.$ : $x \in M\}$. Temos então que $M[G]$ é um $\in$-modelo de domínio enumerável e, ainda, transitivo: dados $x \in b^{G}$, por definição $x=a^{G}$ para algum $a \in^{G} b$; como $a \in^{G} b \leftrightarrow \exists p \in G(\langle a, p\rangle \in b)$ e $M$ é transitivo, temos $a \in M$, donde $x=a^{G} \in M[G]$.

Antes de definirmos qual deve ser a interpretação dos objetos de segunda ordem, vamos ver se $M[G]$ preserva, em algum sentido, o conjunto $G$ o os elementos de $M$, como nós tínhamos inicialmente com $\in^{G}$. É, talvez, de se esperar que sim, por conta do modo que construímos $M[G]$ a partir de $\epsilon^{G}$. A partir de agora, como $(-) \mapsto(-)^{G}$ é uma função definida em $M$ que é, por definição, sobrejetora em $M[G]$, dado $a^{G} \in M[G]$, vamos chamar $a$ de um nome para $a^{G}$; obviamente não há necessidade de haver um único nome em $M$ para 
um elemento fixo de $M[G]$. Assim, para mostrar que $M \subseteq M[G]$, basta que dado $b \in M$, achemos um nome $\hat{b}$ para $b$, isto é, um $\hat{b} \in M$ tal que $b=\overline{\hat{b}}^{G} \in M[G]$.

Agora, dado um $c^{G}$ qualquer, como $c^{G}=\left\{x^{G}: \exists p \in G(\langle x, p\rangle \in c)\right\}$, a parte de $c$ que tomamos para formar $c^{G}$ é o subconjunto $\hat{c} \subseteq c$ tal que $x \in \hat{c} \rightarrow x=\langle y, p\rangle$ para algum $p \in G$. Então, vamos tentar tomar um certo $\hat{b}$ para formar $b$; $\hat{b}$ deve ser alguém que, interpretando seu elementos, teremos os elementos de $b$, isto é, queremos que

$$
b=\hat{b}^{G}=\left\{a^{G}: \exists p(\langle a, p\rangle \in \hat{b})\right\} .
$$

Para facilitar, como $1 \in G$, vamos tomar

$$
b=\left\{a^{G}:\langle a, 1\rangle \in \hat{b}\right\} .
$$

Então $\hat{b}$ é alguém do tamanho de $b$ tal que $\langle a, 1\rangle \in \hat{b} \leftrightarrow a^{G} \in b$. Como $M[G]$ é construído por recursão, vamos tentar achar $b$ por recursão: suponha que se $x \in b$, então $\hat{x}^{G}=x$. Neste caso, se

$$
\hat{b}=\{\langle\hat{x}, 1\rangle: x \in b\}
$$

Então

$$
\begin{aligned}
\hat{b}^{G} & =\left\{a^{G}: \exists p \in G(\langle a, p\rangle \in \hat{b})\right\} \text { (por definição de }(-)^{G} \text { ) } \\
& =\left\{\hat{x}^{G}: x \in b\right\} \text { (pela hipótese sobre } \hat{b} \text { ) } \\
& =\{x: x \in b\} \text { (pela hipótese de indução). }
\end{aligned}
$$

Agora, pondo $G^{\prime}=\{\langle\hat{p}, p\rangle: p \in C\} \in M$, então $G^{\prime}$ é como o conjunto $\operatorname{diag}(C \times C)$ que tínhamos usado antes para representar $G$, e é claro que $G^{\prime G}=G$. Vamos por algumas destas ideias numa definição.

Definição 2.2.5 Sejam $M=\left\langle|M|, R^{H}, F^{H}, \in\right\rangle$ um $\in$-modelo transitivo e enumerável de ZF2 (podemos tomá-lo enumerável por conta de Löwenheim-Skolem, 1.3.19), C uma noção de forcing em $M$ e $G$ um genérico sobre $M$. Se $a, b \in|M|$, definimos

$$
a \in^{G} b \leftrightarrow \exists p(p \in G \wedge\langle a, p\rangle \in b)
$$

e, por indução transfinita no rank de b,

$$
b^{G}=\left\{a^{G}: a \in^{G} b\right\},
$$

(de tal forma que o domínio do modelo $M[G]$ será dado pelos $x^{G}$ ). Se $R$ for uma relação $n$-ária que está em $R^{H}$, defina

$$
R^{G}=\left\{\left\langle x_{1}^{G}, \ldots, x_{n}^{G}\right\rangle: \exists p \in G \text { tal que }\left\langle\left\langle x_{1}, p\right\rangle, \ldots,\left\langle x_{n}, p\right\rangle\right\rangle \in R\right\}
$$

e, se f é uma função n-ária que está em $F^{H}$, defina

$$
\begin{gathered}
\tilde{f}=\left\{\left\langle\left\langle x_{1}, p\right\rangle, \ldots,\left\langle x_{n}, p\right\rangle,\left\langle f\left(x_{1}, \ldots, x_{n}\right), p\right\rangle\right\rangle: p \in C\right\}, \\
f^{G}=\left\{\left\langle x_{1}^{G}, \ldots, x_{n+1}^{G}\right\rangle: \exists p \in G \text { tal que }\left\langle\left\langle x_{1}, p\right\rangle, \ldots,\left\langle x_{n+1}, p\right\rangle\right\rangle \in \tilde{f}\right\}
\end{gathered}
$$

(observe que, desta forma, $(f x)^{G}=f^{G}\left(x^{G}\right)$ ). Definimos então um $\in$-modelo $M[G]$ do seguinte modo: $|M[G]|=\left\{a^{G}: a \in|M|\right\}, R[G]^{H}=\left\{P^{G}: P \in R^{H}\right\}$ e $F[G]^{H}=\left\{f^{G}: f \in\right.$ $\left.F^{H}\right\}$. 
Vamos agora dar a definição de $p \Vdash \varphi$ para $\varphi$ atômica, começando pelo caso $p \Vdash a \in b$. Queremos que a relação de forcing seja definível em $M$ e que no caso em que $p \in G$, então $a \in b$ seja verdadeira em $M[G]$, isto é, que $a^{G} \in b^{G}$. Como $a^{G} \in b^{G} \leftrightarrow a \in^{G} b$, podemos pensar em definir simplesmente

$$
p \Vdash a \in b \Leftrightarrow\langle a, p\rangle \in b,
$$

e daí se $p \in G$, então $a^{G} \in b^{G}$. Contudo, também queremos que se $p$ força $\varphi$, então qualquer extensão de $p$ também force $\varphi$, isto é, se $q \leq p$, então $q$ aproxima $G$ melhor que $p$. Então a definição precisa ser

$$
p \Vdash a \in b \Leftrightarrow \forall q \leq p(\langle a, q\rangle \in b),
$$

ou, ainda, algo menos exigente, e mais razoável:

$$
p \Vdash a \in b \Leftrightarrow \forall q \leq p \exists r \leq q(\langle a, r\rangle \in b) .
$$

Assim, "metade" da definição está pronta: se $p \Vdash a \in b$, então $a^{G} \in b^{G}$. Mas nós queremos algo mais da relação de forcing, a saber: se $a^{G} \in b^{G}$, então deve existir $p \in G$ tal que $p \Vdash a \in b$ e, com esta definição, isto não segue: suponha que $b=\{\langle a, 1\rangle\}$; então $a^{G} \in b^{G}$ mas não vale que $1 \Vdash a \in b$, se $C \neq\{1\}$. Para corrigir isto, vamos acrescentar à definição a possibilidade de que exista algum outro elemento de $b$ que não seja nosso $\langle a, r\rangle$ mas que, sob a interpretação $(-)^{G}$, seja igual a $a^{G}$ :

$$
p \Vdash a \in b \Leftrightarrow \forall q \leq p \exists r \leq q \exists s \geq r \exists x \in M(\langle x, s\rangle \in b \wedge r \Vdash x=a) .
$$

Tomamos $s \geq r$ pois para que $x^{G} \in b^{G}$ basta que exista $s \in G$ com $\langle x, s\rangle \in b$ e, como devemos ter $r \in G$, se $s \geq r$, temos $s \in G$. E, para que $x^{G}=a^{G}$, basta que exista um $r^{\prime} \in G$ tal que $r^{\prime} \Vdash x=a$, e é claro que podemos tomar $r=r^{\prime}$. Nós, no entanto, caímos no problema de que agora a definição de $p \Vdash a \in b$ depende da definição de $p \Vdash x=a$; mas vamos conseguir contornar isto na definição de $p \Vdash a=b$.

A maneira mais natural de definir $p \Vdash a=b$ é, apesar de tudo, em cima da definição de $p \Vdash x \in y$, já que queremos $p \Vdash a=b \Leftrightarrow p \Vdash \forall x(x \in a \leftrightarrow x \in b)$. Assim, vamos ver como fica a definição de $p \Vdash a \subseteq b$; deveria ser para todo $x, p \Vdash x \in a \Rightarrow p \Vdash x \in b$, isto é, para todo $x$,

$$
\begin{aligned}
& \forall q \leq p \exists r \leq q \exists s \geq r \exists x^{\prime} \in M\left(\left\langle x^{\prime}, s\right\rangle \in a \wedge r \Vdash x^{\prime}=x\right) \Rightarrow \\
& \forall q \leq p \exists r^{\prime} \leq q \exists s^{\prime} \geq r^{\prime} \exists y^{\prime} \in M\left(\left\langle y^{\prime}, s^{\prime}\right\rangle \in a \wedge r^{\prime} \Vdash y^{\prime}=x\right),
\end{aligned}
$$

que equivale a (observe que como $r, r^{\prime} \in G$, podemos tomar $r \leq r^{\prime}$ e, na verdade, $r=r^{\prime}$ ):

$$
\begin{array}{r}
\forall q \leq p \exists r \leq q\left[\exists x^{\prime} \in M \exists s \geq r\left(\left\langle x^{\prime}, s\right\rangle a \wedge r \Vdash x^{\prime}=x\right) \rightarrow\right. \\
\left.\exists s^{\prime} \geq r \exists y \in M\left(\left\langle y, s^{\prime}\right\rangle \in b \wedge r \Vdash y=x\right)\right] .
\end{array}
$$

Como queremos, na verdade, que $p \Vdash \forall x(x \in a \rightarrow x \in b)$, ficamos com

$$
\begin{gathered}
\forall x \in M \forall q \leq p \exists r \leq q\left[\exists x^{\prime} \in M \exists s \geq r\left(\left\langle x^{\prime}, s\right\rangle \in a \wedge r \Vdash x^{\prime}=x\right) \rightarrow\right. \\
\left.\exists s^{\prime} \geq r \exists y \in M\left(\left\langle y, s^{\prime}\right\rangle \in b \wedge r \Vdash y=x\right)\right]
\end{gathered}
$$

que, por sua vez, equivale a 


$$
\begin{aligned}
\forall x \in M \forall x^{\prime} \in M \forall q \leq p \exists r \leq q & {\left[\exists s \geq r\left(\left\langle x^{\prime}, s\right\rangle \in a \wedge r \Vdash x^{\prime}=x\right) \rightarrow\right.} \\
\exists s^{\prime} & \left.\geq r \exists y \in M\left(\left\langle y, s^{\prime}\right\rangle \in b \wedge r \Vdash y=x\right)\right] .
\end{aligned}
$$

Agora, como $r \Vdash x^{\prime}=x \wedge r \Vdash y=x \Leftrightarrow r \Vdash y=x^{\prime}$, isto equivale a

$$
\begin{aligned}
\forall x \in M \forall x^{\prime} \in M \forall q \leq p \exists r \leq q[ & {\left[\exists s \geq r\left(\left\langle x^{\prime}, s\right\rangle \in a \wedge r \Vdash x^{\prime}=x\right) \rightarrow\right.} \\
& \left.\exists s^{\prime} \geq r \exists y \in M\left(\left\langle y, s^{\prime}\right\rangle \in b \wedge r \Vdash y=x^{\prime}\right)\right] .
\end{aligned}
$$

Fazendo algumas simplificações, ficamos com nossa forma final:

$$
\forall x \in M \forall q \leq p \exists r \leq q\left[\exists s \geq r(\langle x, s\rangle \in a) \rightarrow \exists s^{\prime} \geq r \exists y \in M\left(\left\langle y, s^{\prime}\right\rangle \in b \wedge r \Vdash y=x\right)\right] .
$$

Assim, é isso que deve significar $p \Vdash a \subseteq b$. Antes de nos opormos ao fato de que $p \Vdash a \subseteq b$ depende de $p \Vdash x=y$, notemos que o rank de $x$ e de $y$ são menores que o maior dos ranks de $a$ e $b$. Como $p \Vdash a=b \Leftrightarrow p \Vdash a \subseteq b \wedge b \subseteq a$, poderemos definir $p \Vdash a=b$ por indução no rank.

Definição 2.2.6 Suponha que $V L(\varphi) \subseteq\{X, f, x\}$ e que todas as variáveis tomem valores em $M$, que $P \in R^{H}, g \in F^{H}, c, d \in M$ (isto é, que $P_{1}, \ldots, P_{n} \in R^{H}$ etc), que $C$ é uma noção de forcing em $M$ e que $p, q, r, s, s^{\prime} \in C$. Estenda o vocabulário de $Z F 2$ (que é formado apenas pelos simbolos $d o \in e$ do $=$ ) adicionando um simbolo de constante para cada $x \in M$, cada $X \in R^{H}$ e cada $f \in F^{H}$ formando assim um novo vocabulário $V^{\prime} ; V^{\prime}$ será usado apenas nas fórmulas que serão "forçadas". Definimos a relação $p$ força a sentença $\varphi(P, g, a)$, em simbolos $p \Vdash \varphi(P, g, a)$, por indução na complexidade de $\varphi(P, g, a)$ :

(a) $p \Vdash c=d$ se, e somente se,

$$
\begin{gathered}
\forall x \forall q \leq p \exists r \leq p\left[\exists s \geq r(\langle x, s\rangle \in a) \rightarrow \exists y \exists s^{\prime} \geq r\left(r \Vdash x=y \wedge\left\langle y, s^{\prime}\right\rangle \in b\right) \wedge\right. \\
\left.\exists t \geq r(\langle x, t\rangle \in b) \rightarrow \exists z \exists t^{\prime} \geq r\left(r \Vdash x=y \wedge\left\langle y, t^{\prime}\right\rangle \in a\right)\right] .
\end{gathered}
$$

Chamaremos a subfórmula $\exists s \geq r(\langle x, s\rangle \in a) \rightarrow \exists y \exists s^{\prime} \geq r\left(r \Vdash x=y \wedge\left\langle y, s^{\prime}\right\rangle \in b\right) d e$ primeira implicação de $p \Vdash c=d, e \exists t \geq r(\langle x, t\rangle \in b) \rightarrow \exists z \exists t^{\prime} \geq r\left(r \Vdash x=y \wedge\left\langle y, t^{\prime}\right\rangle \in a\right)$ de segunda implicação.

(b) $p \Vdash c \in d$ se, e somente se,

$$
\forall q \leq p \exists r \leq q \exists s \geq r \exists x(\langle x, s\rangle \in d \wedge r \Vdash x=c) .
$$

(c) $p \Vdash P\left(a_{1}, \ldots, a_{n}\right)$ se, e somente se,

$\left.\forall q \leq p \exists r \leq q \exists s \geq r \exists x_{1}, \ldots, x_{n}\left(\left\langle x_{1}, s\right\rangle, \ldots,\left\langle x_{n}, s\right\rangle\right\rangle \in P \wedge r \Vdash x_{1}=a_{1} \wedge \ldots \wedge r \Vdash x_{n}=a_{n}\right)$.

(d) $p \Vdash \neg \psi(P, g, a)$ se, e somente se, $\forall q \leq p \neg(q \Vdash \psi(P, g, a))$.

$(e) p \Vdash(\psi \wedge \chi)(P, g, a)$ se, e somente se, $p \Vdash \psi(P, g, a)$ e $p \Vdash \chi(P, g, a)$.

(f) se $x$ é uma variável de primeira ordem, então

$$
p \Vdash \forall x \psi(P, g, a) \Leftrightarrow \forall x \in M(p \Vdash \psi(P, g, a)) .
$$

(g) se X é uma variável de relação $n$-ária, então

$$
p \Vdash \forall X \psi(P, g, a) \Leftrightarrow \forall X\left(X \in R_{n}^{H} \rightarrow p \Vdash \psi(P, g, a)\right) .
$$

(h) se f é uma variável de função $n$-ária, então 


$$
p \Vdash \forall f \psi(P, g, a) \Leftrightarrow \forall f\left(f \in F_{n}^{H} \rightarrow p \Vdash \psi(P, g, a)\right) .
$$

Agora, dois lemas que seguem imediatamente da definição. O primeiro é apenas o fato de que se $q$ estende $p$, então $q$ força aquilo que já sabíamos que $p$ forçava e o segundo diz que a relação de forcing, em relação a uma única fórmula $\varphi$, é definível em $M$. Prova-se por indução na complexidade de $\varphi$.

Lema 2.2.7 Se $p \Vdash \varphi$ e $q \leq p$, então $q \Vdash \varphi$.

Lema 2.2.8 Se $V L(\varphi)=\{X, f, x\}$, então existe uma fórmula $\psi_{\varphi} \operatorname{com} V L\left(\psi_{\varphi}\right)=\{y, w, z, X, f, x\}$ tal que

$$
p \Vdash \varphi(P, g, a) \Leftrightarrow M \vDash \psi_{\varphi}[p, C, \leq, P, g, a] .
$$

O próximo resultado é fundamental. A parte $(i)$ diz que se $p \Vdash \varphi$, então $M[G]$ precisa satisfazer $\varphi$, onde $G$ é um genérico arbitrário sobre $M$ que contém $p$; a parte $(i i)$ diz que, se $M[G]$ satisfaz $\varphi$, então de fato existe uma condição em $G$ que força $\varphi$.

Teorema 2.2.9 $(i) p \Vdash \varphi(P, g, a) \Leftrightarrow$ para todo genérico $G$ com $p \in G$, temos $M[G] \vDash$ $\varphi\left[P^{G}, g^{G}, a^{G}\right]$ (onde $a^{G}=a_{1}^{G}, \ldots, a_{n}^{G}$, etc).

(ii) Se $M[G] \vDash \varphi\left[P^{G}, g^{G}, a^{G}\right]$, então existe $p \in G$ tal que $p \Vdash \varphi(P, g, a)$.

Prova. Vamos provar $(i)$ e $(i i)$ simultâneamente por indução na complexidade de $\varphi(P, g, a)$.

Fórmulas atômicas. Suponha que $\varphi(P, g, a)$ é da forma $c=d$, para certos símbolos de constante $c$ e $d$ de $V^{\prime}$, e que $(i)$ e (ii) são válidos para conjuntos de rank menor que $\max \{\operatorname{rank}(c), \operatorname{rank}(d)\}$. Então

$$
\begin{aligned}
& M[G] \vDash c=d \Leftrightarrow c^{G}=d^{G} \\
& \Leftrightarrow \quad \forall x^{G} \in M[G]\left(x^{G} \in c^{G} \leftrightarrow x^{G} \in d^{G}\right) \\
& \Leftrightarrow \quad \forall x \in M\left(x \in^{G} c \rightarrow \exists y \in M\left(y \in \in^{G} d \wedge y^{G}=x^{G}\right) \wedge\right. \\
&\left.\quad x \in^{G} d \rightarrow \exists z \in M\left(z \in^{G} c \wedge z^{G}=x^{G}\right)\right),
\end{aligned}
$$

que equivale, por hipótese de indução, à forma que iremos usar:

$$
\begin{gathered}
\forall x \in M\left[\exists s \in G(\langle x, s\rangle \in c) \rightarrow \exists y \in M \exists r \in G \exists s^{\prime} \geq r\left(\left\langle y, s^{\prime}\right\rangle \in d \wedge r \Vdash x=y\right) \wedge\right. \\
\left.\exists t \in G(\langle x, t\rangle \in d) \rightarrow \exists z \in M \exists q \in G \exists t^{\prime} \geq q\left(\left\langle z, t^{\prime}\right\rangle \in c \wedge q \Vdash x=z\right)\right] .
\end{gathered}
$$

Vamos provar $(i)$ primeiro. Suponha que $p \Vdash c=d$, que $G$ é um genérico com $p \in G$, que $x \in M$ e que $\exists s \in G(\langle x, s\rangle \in c)$. Seja $q \leq p, s$ e considere, em $M$, o conjunto

$$
D=\left\{r \leq q: \exists s \geq r(\langle x, s\rangle \in c) \rightarrow \exists y \exists s^{\prime} \geq r\left(\left\langle y, s^{\prime}\right\rangle \in d \wedge r \Vdash x=y\right)\right\} .
$$

Como $p \Vdash c=d, D$ é denso abaixo de $q$. Observe agora que

Lema 2.2.10 Se $G$ é um genérico tal que $p \in G$ e $D$ é denso abaixo de $p$, então $G \cap D \neq \emptyset$.

Prova. Seja $D^{\prime}=D \cup\{q: q$ não é compatível com $p\}$. Então $D^{\prime}$ é denso em $M$ : dado $r \in C$, se $r$ é compatível com $p$, seja $s \leq p, r$; como $s \leq p$ e $D$ é denso abaixo de $p$, existe $s^{\prime} \leq s$ em $D \subseteq D^{\prime}$; se $r$ não é compatível com $p$, então $r \in D^{\prime}$ e assim $D^{\prime}$ é denso. Agora, como $p \in G$ e $G$ é genérico, temos $G \cap\{q: q$ não é compatível com $p\}=\emptyset$, e portanto $G \cap D \neq \emptyset$.

Tome então $r \in D \cap G$; daí $r \leq q \leq s$, e assim existe $s \geq r$ tal que $\langle x, s\rangle \in c$, e portanto por definição de $D$, 


$$
\exists y \in M \exists r \in G \exists s^{\prime} \geq r\left(\left\langle y, s^{\prime}\right\rangle \in d \wedge r \Vdash x=y\right),
$$

e assim vale uma das implicações de $(*)$. Mas é claro que se tomarmos $D^{\prime}=\{r \leq q: \exists t \geq$ $\left.r(\langle x, t\rangle \in d) \rightarrow \exists z \exists t^{\prime} \geq r\left(\left\langle z, t^{\prime}\right\rangle \in c \wedge r \Vdash x=z\right)\right\}$ podemos provar da mesma forma que vale a outra implicação de $(*)$.

Suponha agora que para todo genérico $G$ com $p \in G$ vale $(*)$ e seja $q \leq p$. Se $\neg \exists s \geq$ $q(\langle x, s\rangle \in c) \wedge \neg \exists t \geq q(\langle x, t\rangle \in d)$, tomo $r=q$ na definição de $p \Vdash c=d$. Se $\neg \exists s \geq q(\langle x, s\rangle \in$ $c)$, seja $G$ um genérico com $q \in G$. Como $q \leq p, s$, temos $p, s \in G$. Logo, $\exists s \in G(\langle x, s\rangle \in c)$ e, portanto, por $(*)$,

$$
\exists y \in M \exists r \in G \exists s^{\prime} \geq r\left(\left\langle y, s^{\prime}\right\rangle \in d \wedge r \Vdash x=y\right) .
$$

Como $r, q \in G$, podemos tomar $r \leq q$, donde temos a primeira implicação de $p \Vdash c=d$. Se $\neg \exists t \geq q(\langle x, t\rangle \in d)$, está demonstrado e, caso contrário, procedemos como no caso em que $\exists s \geq q(\langle x, s\rangle \in c)$.

Vamos provar (ii) agora. Suponha que $G$ é um genérico satisfazendo (*) e defina em $M$

$$
\begin{aligned}
D=\{q \in C: \quad & \exists x \forall r \leq q\left(\left[\exists s \geq r(\langle x, s\rangle \in c) \wedge \forall y \forall s^{\prime} \geq r\left(r \Vdash x=y \rightarrow\left\langle y, s^{\prime}\right\rangle \notin d\right)\right] \vee\right. \\
& {\left.\left[\exists t \geq r(\langle x, t\rangle \in d) \wedge \forall z \forall t^{\prime} \geq r\left(r \Vdash x=y \rightarrow\left\langle y, t^{\prime}\right\rangle \notin c\right)\right]\right) \vee } \\
& q \Vdash c=d\} .
\end{aligned}
$$

Então $D$ é denso em $M$, pois dado $p \in C$ se nenhum $q \leq p$ satisfaz o lado esquerdo da disjunção, então $p \Vdash c=d$. Tome agora $q \in D \cap G$; suponha por absurdo que

$$
\exists x \in M \forall r \leq q\left[\exists s \geq r(\langle x, s\rangle \in c) \wedge \forall y \forall s^{\prime} \geq r\left(r \Vdash x=y \rightarrow\left\langle y, s^{\prime}\right\rangle \notin d\right)\right] .
$$

Logo, dado um tal $x$, para todo $r \leq q, \exists s \geq q(\langle x, s\rangle \in c)$. Vamos mostrar que $c^{G} \neq$ $d^{G}$, contrariando a hipótese $(*)$. Seja $x^{G} \in c^{G}$; vamos mostrar que $x^{G} \in d^{G}$ leva a uma contradição: neste caso $\exists y \in M \exists q_{0} \in G\left(\left\langle y, q_{0}\right\rangle \in d \wedge y^{G}=x^{G}\right)$. Por hipótese de indução $\exists q_{1} \in G\left(q_{1} \Vdash x=y\right)$. Como $r$ é arbitrário menor ou igual a $q$, podemos tomar $r \leq q_{1} \leq q_{0}$; mas então $r \leq q_{0}$ donde $\left\langle y, q_{0}\right\rangle \notin d$, pela hipótese sobre $x$, uma contradição. Assim $x^{G} \in$ $c^{G}-d^{G}$. De modo análogo provamos que $x$ também não pode satisfazer $[\exists t \geq r(\langle x, t\rangle \in$ d) $\left.\wedge \forall z \forall t^{\prime} \geq r\left(r \Vdash x=y \rightarrow\left\langle y, t^{\prime}\right\rangle \notin c\right)\right]$, donde $q \Vdash c=d$.

Assuma agora que $(i)$ e (ii) são válidos para $c=d$ e vamos prová-los para $c \in d$. Temos que

$$
M[G] \vDash c \in d \Leftrightarrow c \in^{G} d \Leftrightarrow \exists x \in M\left(x \in^{G} d \wedge x^{G}=c^{G}\right),
$$

que por hipótese de indução é equivalente a

$$
(* *) \quad \exists x \in M \exists r \in G \exists s \geq r(\langle x, s\rangle \in d \wedge r \Vdash x=c) .
$$

Para provar $(i)$, suponha que $p \Vdash c \in d$. Dado $G$ um genérico com $p \in G$, como $p \Vdash c \in d$, o conjunto

$$
D=\{r \in C: \exists x \exists s \geq r(\langle x, s\rangle \in d \wedge r \Vdash x=c)\}
$$

é denso abaixo de $p$ e pertence a $M$. Tome então $r \in D \cap G$; tal $r$ satisfaz (**). Suponha agora que $(* *)$ é válido para todo genérico $G$ tal que $p \in G$ e tome $q \leq p$; tome também um genérico $G^{\prime}$ tal que $q \in G^{\prime}$. Assim, $p \in G^{\prime}$ e podemos tomar o $r$ em $(* *)$ de tal forma que $r \leq q$, e então $p \Vdash c \in d$.

Para provar (ii), suponha (**) e tome um $r$ fornecido por esta hipótese. Dado um genérico $G^{\prime} \operatorname{com} r \in G^{\prime}$ temos que $c^{G^{\prime}} \in d^{G^{\prime}}$. Assim, por $(i), r \Vdash c \in d$.

Suponha agora que $\varphi(P, g, a)$ é da forma $P\left(a_{1}, \ldots, a_{n}\right)$, e vamos provar $(i)$ neste caso. É análogo ao caso $c \in d$ : suponha que $p \Vdash P\left(a_{1}, \ldots, a_{n}\right)$ e que $G$ é um genérico com $p \in G$. Pela hipótese de indução, $M[G] \vDash P\left(a_{1}, \ldots, a_{n}\right) \Leftrightarrow$ 
$\exists x_{1}, \ldots, x_{n} \in M \exists r \in G \exists p \geq r\left(\left\langle\left\langle x_{1}, p\right\rangle, \ldots,\left\langle x_{n}, p\right\rangle\right\rangle \in P \wedge r \Vdash x_{1}=a_{1} \wedge \ldots \wedge r \Vdash x_{n}=a_{n}\right)$.

Como $p \Vdash P\left(a_{1}, \ldots, a_{n}\right)$, o conjunto

$D=\left\{r \in C: \exists p \geq r \exists x_{1}, \ldots, x_{n}\left(\left\langle\left\langle x_{1}, p\right\rangle, \ldots,\left\langle x_{n}, p\right\rangle\right\rangle \in P \wedge r \Vdash x_{1}=a_{1} \wedge \ldots \wedge r \Vdash x_{n}=a_{n}\right)\right\}$

é denso abaixo de $p$, e assim existe $r \in D \cap G$. A prova do outro lado da equivalência, bem como a de (ii), são tão análogas que vamos omití-las.

Passo de indução da conjunção. $(i): p \Vdash \psi \wedge \chi(P, g, a) \Leftrightarrow p \Vdash \psi(P, g, a)$ e $p \Vdash \chi(P, g, a) \Leftrightarrow$ $\forall G \overline{\text { genérico tal que } p \in G \text { temos }} M[G] \vDash \psi\left[P^{G}, g^{G}, a^{G}\right]$ e $\forall G$ genérico tal que $p \in G$ temos $\left.M[G] \vDash \chi\left[P^{G}, g^{G}, a^{G}\right]\right) \Leftrightarrow \forall G$ genérico tal que $p \in G$ temos $\left.M[G] \vDash(\psi \wedge \chi)\left[P^{G}, g^{G}, a^{G}\right]\right)$.

(ii) Suponha que $M[G] \vDash(\psi \wedge \chi)\left[P^{G}, g^{G}, a^{G}\right]$; então $M[G] \vDash \psi\left[P^{G}, g^{G}, a^{G}\right]$ e $M[G] \vDash$ $\chi\left[P^{G}, g^{G}, a^{G}\right]$. Por hipótese de indução $\exists r, s \in G$ tais que $r \Vdash \psi(P, g, a)$ e $s \Vdash \chi(P, g, a)$; como $r, s \in G, \exists p \leq r, s$ e aí $p \Vdash(\psi \wedge \chi)(P, g, a)$.

Passo de indução da negação. $(i)$ : Se $p \Vdash \neg \psi(P, g, a)$, suponha por absurdo que $M[G] \vDash$

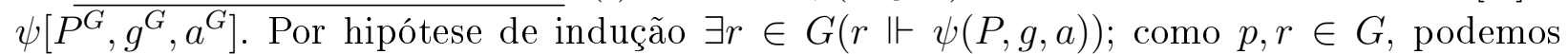
tomar $r \leq p$, um absurdo com $p \Vdash \neg \psi(P, g, a)$. Se $\forall G$ genérico tal que $p \in G$ temos $M[G] \not \models$ $\psi\left[P^{G}, g^{G}, a^{G}\right]$, suponha que $\neg(p \Vdash \neg \psi)$, isto é, que $\exists q \leq p(q \Vdash \psi(P, g, a))$. Seja $G^{\prime}$ tal que $q \in G^{\prime}$; como $q \leq p$, temos $p \in G^{\prime}$. Mas $q \Vdash \psi(P, g, a)$ implica, por hipótese de indução, que $M\left[G^{\prime}\right] \vDash \psi\left[P^{G^{\prime}}, g^{G^{\prime}}, a^{G^{\prime}}\right]$ e $p \in G^{\prime}$ implica, pela hipótese em $p$, que $M\left[G^{\prime}\right] \not \models \psi\left[P^{G^{\prime}}, g^{G^{\prime}}, a^{G^{\prime}}\right]$, um absurdo.

(ii): Suponha $M[G] \vDash \neg \psi(P, g, a)$; então, por hipótese de indução, se $p \in G$ temos $\neg(p \Vdash \psi(P, g, a))$. Seja $D=\{q: q \Vdash \psi(P, g, a) \vee q \Vdash \neg \psi(P, g, a)\}$; como $p \Vdash \psi(P, g, a)$ ou $\exists q \leq p(q \Vdash \psi(P, g, a)), D$ é denso abaixo de $p$, e portanto existe $r \in D \cap G$. Como $r \in G$, temos $r \Vdash \neg \psi(P, g, a)$.

Passo de indução para quantificadores de primeira ordem. $(i)$ :

\begin{aligned} \hline$p \Vdash \forall x \psi(P, g, a) & \Leftrightarrow \forall x \in M(p \Vdash \psi(P, g, a)) \\ & \Leftrightarrow \forall x \in M \forall G\left(G\right.$ é genérico e $\left.p \in G \rightarrow M[G] \vDash \psi\left[P^{G}, g^{G}, a^{G}\right]\right) \\ & \Leftrightarrow \forall x^{G} \in M[G] \forall G\left(G\right.$ é genérico e $\left.p \in G \rightarrow M[G] \vDash \psi\left[P^{G}, g^{G}, a^{G}\right]\right) \\ & \Leftrightarrow \forall G\left(G\right.$ é genérico e $\left.p \in G \rightarrow \forall x^{G} \in M[G]\left(M[G] \vDash \psi\left[P^{G}, g^{G}, a^{G}\right]\right)\right) \\ & \Leftrightarrow \forall G(G$ é genérico e $p \in G \rightarrow M[G] \vDash \forall x \psi)\end{aligned}$.

(ii): Seja em $M$

$$
D=\{r \in C: \exists x(r \Vdash \neg \psi(P, g, a)) \vee \forall x(r \Vdash \psi(P, g, a))\} .
$$

Então $D$ é denso em $M$ : dado $r \in C$, se $\neg \forall q \leq r \exists x \in M(q \Vdash \neg \psi(P, g, a))$, isto é, se nenhum $q \leq r$ força $\neg \psi(P, g, a)$ para nenhum $x \in M$, como $r \Vdash \neg \neg \psi(P, g, a) \Leftrightarrow \forall q \leq r \neg(q \Vdash$ $\neg \psi(P, g, a)$ por definição, temos $\forall x \in M(r \Vdash \neg \neg \psi(P, g, a))$. Mas então, pela hipótese de indução $(i)$,

$$
\forall x \in M(r \Vdash \psi(P, g, a)) .
$$

Suponha então que $M[G] \vDash \forall x \psi\left[P^{G}, g^{G}, a^{G}\right]$. Seja $p \in D \cap G$ e suponha que $\exists x \in M(p \Vdash$ $\neg \psi(P, g, a)$. Como $M[G] \vDash \forall x \psi\left[P^{G}, g^{G}, a^{G}\right]$, dado $x \in M, M[G] \vDash \psi\left[P^{G}, g^{G}, a^{G}\right]$, e assim $\exists q \in G(q \Vdash \psi(P, g, a))$. Mas aí $\exists r \in G, r \leq p, q$ tal que $r \Vdash(\psi \wedge \neg \psi)(P, g, a)$.

O passo de indução para o quantificador de segunda ordem de relações é semelhante ao caso do quantificador de primeira ordem, e vamos omití-lo.

M[G] é modelo de ZF2. Vamos passar agora à prova de que $M[G]$ é um modelo de Henkin correto para o sistema dedutivo $D 2+$ e é tal que $M[G] \vDash Z F 2$. Até o final desta seção, suponha as hipóteses do teorema a seguir: 
Teorema 2.2.11 Sejam $M$ um $\in$-modelo transitivo enumerável de $Z F 2$ correto para o sistema dedutivo $D 2+,\langle C, \leq\rangle$ uma noção de forcing em $M$ e $G \subseteq C$ genérico sobre $M$. Então $M[G]$ é um $\in$-modelo transitivo enumerável de $Z F 2$ correto para D2+ com os mesmos ordinais que $M, M \subseteq M[G]$ e $G \in M[G]$. Além disso, é o menor modelo com tais propriedades.

Prova. Do fato de $M[G]$ ser $\in$-modelo transitivo segue que $M[G]$ satisfaz os axiomas da extensionalidade e da regularidade. A prova do teorema consistirá de uma série de lemas; vamos começar mostrando que $M[G]$ é correto para $D 2+$.

Lema 2.2.12 Dada $\varphi$ tal que $V L(\varphi)=\left\{x_{1}, \ldots, x_{n}\right\}$, existe $A^{G} \in R[G]^{H}$ tal que

$$
M[G] \vDash \varphi\left[a_{1}^{G}, \ldots, a_{n}^{G}\right] \Leftrightarrow\left\langle a_{1}^{G}, \ldots, a_{n}^{G}\right\rangle \in A^{G} .
$$

Prova. Como $M$ é correto para $D 2+$, existe $A \in R^{H}$ tal que

$$
\left\langle\left\langle x_{1}, p\right\rangle, \ldots,\left\langle x_{n}, p\right\rangle\right\rangle \in A \Leftrightarrow p \in C \wedge p \Vdash \varphi\left(x_{1}, \ldots, x_{n}\right) .
$$

Então $\left\langle x_{1}^{G}, \ldots, x_{n}^{G}\right\rangle \in A^{G} \Rightarrow \exists p \in G\left(\left\langle\left\langle x_{1}, p\right\rangle, \ldots,\left\langle x_{n}, p\right\rangle\right\rangle \in A\right) \Rightarrow p \Vdash \varphi\left(x_{1}, \ldots, x_{n}\right) \Rightarrow$ $M[G] \vDash \varphi\left[x_{1}^{G}, \ldots, x_{n}^{G}\right]$. Recíprocamente, se $M[G] \vDash \varphi\left[x_{1}^{G}, \ldots, x_{n}^{G}\right]$, então $\exists p \in G(p \Vdash$ $\left.\varphi\left(x_{1}, \ldots, x_{n}\right)\right)$, e daí $\left\langle\left\langle x_{1}, p\right\rangle, \ldots,\left\langle x_{n}, p\right\rangle\right\rangle \in A$, donde $\left\langle x_{1}^{G}, \ldots, x_{n}^{G}\right\rangle \in A^{G}$.

Lema 2.2.13 Suponha que $A^{G} \in R[G]^{H}$ seja uma relação $(n+1)$-ária tal que para todos $x_{1}^{G}, \ldots, x_{n}^{G}$ existe $y^{G}$ tal que $\left\langle x_{1}^{G}, \ldots, x_{n}^{G}, y^{G}\right\rangle \in A^{G}$. Então existe $f^{G} n$-ária em $F[G]^{H}$ tal que para todos $x_{1}^{G}, \ldots, x_{n}^{G}$, temos $\left\langle x_{1}^{G}, \ldots, x_{n}^{G}, f^{G}\left(x_{1}^{G}, \ldots, x_{n}^{G}\right)\right\rangle \in A^{G}$.

Prova. Como $M$ é correto para $D 2+, \exists \tilde{A} \in R^{H}$ tal que $\langle x, y\rangle \in \tilde{A} \Leftrightarrow \exists p \in C(\langle\langle x, p\rangle,\langle y, p\rangle\rangle \in$ $A)$. Considere $\bar{A}=\tilde{A} \cup\left\{\langle x, \emptyset\rangle: x \in M \wedge x \notin \pi_{1} \tilde{A}\right\} \in R^{H}$. Então $\forall x \exists y(\langle x, y\rangle \in \bar{A}) \mathrm{e}$ $\forall x \in \pi_{1} \tilde{A} \exists p \in C(\langle\langle x, p\rangle,\langle f(x), p\rangle\rangle \in A)$. Dado $a \in M[G]$, existe $x \in \pi_{1} \tilde{A}$ tal que $x^{G}=a$; $\operatorname{assim} \exists p \in C\left(\langle\langle x, p\rangle,\langle f(x), p\rangle\rangle \in A\right.$. Logo $\left\langle x^{G},(f(x))^{G}\right\rangle \in A^{G}$, ie, $\left\langle a, f^{G}(a)\right\rangle \in A^{G}$.

Assim, $M[G]$ é correto para $D 2+$. Vamos mostrar agora que $M[G] \vDash Z F 2$.

Lema 2.2.14 Se $A^{G} \in R[G]^{H}$ é unária e tal que $A^{G} \subseteq a^{G}$ para algum $a^{G} \in M[G]$, então existe $c^{G} \in M[G]$ tal que $c^{G}=A^{G}$.

Prova. Tome

$$
c=\{\langle x, p\rangle \in \operatorname{dom}(a) \times C: p \Vdash A(x)\} .
$$

Então $c \in M$. Se $x^{G} \in A^{G}$, então $x^{G} \in a^{G}$, donde $x \in \operatorname{dom}(a)$. Considere

$$
D=\{p \in C: p \Vdash A(x) \vee p \Vdash \neg A(x)\} .
$$

Como $D$ é denso, $\exists p \in D \cap G$. Mas $x^{G} \in A^{G}$, e portanto $p \Vdash A(x)$. Assim, $\langle x, p\rangle \in c$, e aí $x^{G} \in c^{G}$. Se $x^{G} \in c^{G}$, então $\exists p \in G(\langle x, p\rangle \in c \wedge p \Vdash A(x))$, o que implica $x^{G} \in A^{G}$.

Lema 2.2.15 Se $A^{G} \in R[G]^{H}$ é unária e $\exists b \in M\left(\forall x^{G} \in A^{G} \exists y \in b\left(y^{G}=x^{G}\right)\right)$, ent $\tilde{a} o$

$$
\exists c^{G} \forall x^{G}\left(x^{G} \in c^{G} \leftrightarrow x^{G} \in A^{G}\right) .
$$

Em outras palavras, se $A^{G}$ é uma classe de $M[G]$ para a qual existe um $b \in M$ tal que todo elemento de $A^{G}$ tem um nome em $b$, então existe um conjunto em $M[G]$ com a mesma extensão que $A^{G}$. 
Prova. Seja $c=b \times\{1\}$. Vamos provar que $A^{G} \subseteq c^{G}$; o resultado segue do último lema. Se $x^{G} \in A^{G}$, então por hipótese $\exists y \in b\left(y^{G}=x^{G}\right)$. Agora, $\langle y, 1\rangle \in c$; logo $y^{G} \in c^{G}$. Assim $x^{G} \in c^{G}$.

Lema 2.2.16 Se $a^{G} \in M[G]$, então $\bigcup a^{G} \in M[G]$.

Prova. Vamos usar o último lema. Seja $y^{G}$ tal que $\exists x^{G}\left(y^{G} \in x^{G} \in a^{G}\right)$ e tome $b$ o fecho transitivo de $a$; então $b \in M$ e $y \in b$. Pelo axioma da compreensão de segunda ordem, existe $A^{G}$, a classe tal que $z^{G} \in A^{G} \leftrightarrow z^{G} \in \bigcup a^{G}$.

Lema 2.2.17 $M[G]$ satisfaz o axioma das partes, isto é, se $a^{G} \in M[G]$, então $P\left(a^{G}\right) \cap$ $M[G] \in M[G]$.

Prova. Vamos usar o lema 2.2.15 novamente. Seja $b=M \cap P(\operatorname{dom}(a) \times C)$. Então $b \in M$. Defina

$$
z=\{\langle y, p\rangle \in \operatorname{dom}(a) \times C:\langle y, p\rangle \in x\}
$$

Então $z \in b$ e $z^{G}=x^{G}$.

Lema 2.2.18 $M[G]$ satisfaz substituição de segunda ordem.

Prova. Novamente, vamos usar o lema 2.2.15. Seja $f^{G} \in F[G]^{H}$ unária e $a^{G} \in M[G]$; temos que $f_{*}^{G}\left(a^{G}\right) \in R[G]^{H}$. Seja $\varphi$ a fórmula $x_{1} \in x_{2} \wedge f x_{1}=x_{3}$; para cada $x \in \operatorname{dom}(a)$, seja

$$
D_{x}=\{p \in C: \exists w(p \Vdash(\exists y \varphi \rightarrow \varphi)(x, a, w))\} .
$$

$D_{x}$ é denso para cada $x$ : de fato, dado $p \in C$, se $p \Vdash \exists y \varphi(x, a)$, seja $G^{\prime}$ um genérico tal que $p \in G^{\prime}$; então existe $w \in M$ tal que $M\left[G^{\prime}\right] \vDash \varphi\left[x^{G^{\prime}}, a^{G^{\prime}}, w^{G^{\prime}}\right]$, donde existe $q \in G^{\prime}$ com $q \Vdash \varphi(a, x, w)$. Como $p, q \in G^{\prime}$, existe $r \leq p, q, r \in G^{\prime}$, tal que $r \Vdash(\exists y \varphi \rightarrow \varphi)(x, a, w)$, donde $r \in D_{x}$. Caso contrário, isto é, se $\exists q \leq p(q \Vdash \neg \exists y \varphi(x, a))$, então, dado um genérico $G^{\prime}$ com $q \in G^{\prime}$, temos $\neg \exists w \in M\left(M\left[G^{\prime}\right] \vDash \varphi\left[x^{G^{\prime}}, a^{G^{\prime}}, w^{G^{\prime}}\right]\right)$ e, assim, $\exists r \in G^{\prime} \forall w \in M(q \Vdash$ $(\exists y \varphi \rightarrow \varphi)(x, a, w))$. Podemos tomar $r \leq q$ e, como $M \neq \emptyset, r \in D_{x}$.

Considere agora, para cada $x \in \operatorname{dom}(a)$, cada $p \in C$, por substituição,

$$
A_{x, p}=\{w: p \Vdash(\exists y \varphi \rightarrow \varphi)(x, a, w)\} .
$$

Dado $x \in \operatorname{dom}(a)$, existe $p \in G \cap D_{x}$ e, assim, $A_{x, p} \neq \emptyset$; como $M$ satisfaz o axioma da escolha, existe

$$
g \in \prod\left\{A_{x, p}: x \in \operatorname{dom}(a) \wedge p \in C \wedge A_{x, p} \neq \emptyset\right\} .
$$

Tome $w=g\langle x, p\rangle$. Então $p \Vdash(\exists y \varphi \rightarrow \varphi)(x, a, w)$. Como $p \in G, M[G] \vDash \exists y \varphi \rightarrow \varphi\left[x^{G}, a^{G}, w^{G}\right]$. Como $x^{G} \in a^{G}, M[G] \vDash \exists y \varphi\left[x^{G}, a^{G}\right] ; \operatorname{logo} M[G] \vDash \varphi\left[x^{G}, a^{G}, w^{G}\right]$. O resultado segue então pelo lema 2.2.15, com $b=\operatorname{Im}(g)$.

Corolário 2.2.19 $M[G]$ satisfaz o axioma da infinidade.

Prova. Como $\omega \in M$ e $M \subseteq M[G], \omega \in M[G]$; pelo último lema, segue.

Lema 2.2.20 Se $M \subseteq N, G \in N$ e $N \vDash Z F 2$, então $M[G] \subseteq N$.

Prova. Defina, em $N$, por recursão no $\operatorname{rank}(y)$ : 


$$
K(y)=\{K(x): \exists p \in G(\langle x, p\rangle \in y)\} .
$$

Pela transitivadade de $M, K \uparrow M=(-)^{G}$. Seja agora $a^{G} \in M[G]$; então $a^{G}=\{K(x): \exists p \in$ $G(\langle x, p\rangle \in a)\}$. Como $a \in M$ e $M \subseteq N$, temos $a^{G} \in N$.

Lema 2.2.21 $O N^{M[G]}=O N^{M}$.

Prova. Como $M \subseteq M[G]$ e " $x$ é ordinal" é absoluto, $O N^{M} \subseteq O N^{M[G]}$. Suponha agora que $\alpha \in O N^{M[G]}$; como

$$
\operatorname{rank}\left(a^{G}\right) \leq \operatorname{rank}(a)
$$

(a prova é por indução em $a^{G}$ ) e rank é absoluto, seja $a \in M$ tal que $a^{G}=\alpha$. Então $\alpha=$ $\operatorname{rank}(\alpha)=\operatorname{rank}\left(a^{G}\right) \leq \operatorname{rank}(a)$. Como $a \in M$, temos $\operatorname{rank}(a) \in M$ e, pela transitivadade de $M, \alpha \in M$.

\subsection{Um modelo de $\mathrm{ZF} 2+\neg \mathrm{HC}$}

A partir daqui a prova é a mesma que para $Z F$. Em particular, podemos provar também que $Z F 2$ é consistente com $V \neq L$. Vamos fazer isto primeiro.

Proposição 2.3.1 Se $C$ é uma noção de forcing em $M$ tal que para todo $p \in C$ existem $r, q \in C$ tais que $r, q \leq p$ e $r$ e $q$ são incompatíveis, então não existem genéricos sobre $M$ que estejam em $M$.

Prova. Suponha que $G \subseteq C$ é um genérico sobre $M$ e que $G \in M$. Considere $D=C-G$; então $D$ é denso: dado $p \in C$, se $p \notin D$, por hipótese existem $r, q \leq p$ incompatíveis; assim, não podem estar ambos em $G$, donde um deles está em $D$. Mas como $G$ é genérico, precisamos ter $G \cap D \neq \emptyset$, um absurdo com a definição de $D$.

Corolário 2.3.2 $Z F 2+V \neq L$ é consistente.

Prova. Sejam $M$ um $\in$-modelo transitivo enumerável qualquer de $Z F 2+V=L$ e $C$ uma ordem parcial com máximo em $M$, satisfazendo as hipóteses de 2.3.1 (como, por exemplo, o conjunto das funções parciais finitas de $\omega$ em 2 , ordenado por $p \leq q \Leftrightarrow q \subseteq p$ ). Então existe um genérico $G$ sobre $M$ tal que $G \notin M$. Como " $x$ é construtível" é absoluto para $L$ (Shoenfield (1967)) e os ordinais de $M$ são os mesmos de $M[G]$, os construtíveis de $M[G]$ são os mesmos que em $M$. Mas como $G \notin M, G$ é um conjunto não construtível em $M[G]$, donde $M[G] \vDash V \neq L$.

Lema 2.3.3 Seja

$$
\operatorname{Fn}(I, J)=\{p: \operatorname{Fun}(p) \wedge \operatorname{Card}(p)<\omega \wedge \operatorname{dom}(p) \subseteq I \wedge \operatorname{Im}(p) \subseteq J\},
$$

e ordene por $p \leq q \leftrightarrow q \subseteq p$. Então, se $I, J \in M, I$ for infinito, $J \neq \emptyset e G \subseteq F n(I, J)$ é genérico sobre $M$, então $\bigcup G$ é uma função de $I$ em $J$ sobrejetora.

Prova. Como " $x$ é finito" é absoluto, $F n(I, J)^{M}=F n(I, J) \in M$. É claro que $\bigcup G$ é uma função com domínio contido em $I$ e imagem em $J$; agora, dado $j \in J$, como $I$ é infinito, $D_{j}=\{p \in F n(I, J): j \in \operatorname{Im}(p)\}$ é denso em $M$, e além disso $D_{j} \in M$. Logo $G \cap D_{j} \neq \emptyset$ para cada $j$, e assim $\operatorname{Im}(\bigcup G)=J$. Semelhantemente, se $J \neq \emptyset$, então o conjunto $D_{i}=\{p \in F n(I, J): i \in \operatorname{dom}(p)\}$ é denso para todo $i \in I$, e assim $\operatorname{dom}(\bigcup G)=I$. 
Lema 2.3.4 Se $\kappa \in M e G \subseteq F n(\kappa \times \omega, 2)$ é genérico sobre $M$, então $\left(\operatorname{Card}(\kappa) \leq 2^{\omega}\right)^{M[G]}$.

Prova. Temos que $\bigcup G: \kappa \times \omega \rightarrow 2$; defina a sequencia de funções $\left\langle f_{\alpha}: \alpha<\kappa\right\rangle$ como $f_{\alpha}(n)=(\bigcup G)(\alpha, n), n \in \omega$. Veja que as $f_{\alpha}$ são distintas: considere para cada par $\alpha, \beta<\kappa$, $\alpha \neq \beta$,

$$
D_{\alpha \beta}=\{p: \exists n \in \omega(\langle\alpha, n\rangle \in \operatorname{dom}(p) \wedge\langle\beta, n\rangle \in \operatorname{dom}(p) \wedge p(\alpha, n) \neq p(\beta, n))\} ;
$$

então $D_{\alpha \beta}$ é denso em $M$, e portanto $G \cap D_{\alpha \beta} \neq \emptyset$, e assim $f_{\alpha} \neq f_{\beta}$. Mas então $\left\langle f_{\alpha}: \alpha<\kappa\right\rangle$ é uma sequencia de $\kappa$ funções em $M[G]$ com domínio $\omega$ e imagem igual a 2.

Falta ainda mostrar que é possível tomar $\kappa \in M$ de tal forma que $\operatorname{Card}(\kappa)^{M[G]}$ seja não enumerável. Obteremos isto se $F n(I, J)$ satisfazer uma certa propriedade:

Definição 2.3.5 Se $C$ é uma ordem parcial, um $X \subseteq C$ é uma anticadeia se todos os elementos de $X$ são incompativeis entre si.

Lema 2.3.6 Se J é enumerável, então $F n(I, J)$ tem todas as suas anticadeias enumeráveis.

A prova deste lema depende do chamado lema dos $\Delta$-sistemas (que é um caso particular do Teorema de Erdös-Rado):

Lema 2.3.7 Se A é uma família não-enumerável de conjuntos finitos, então existe um $X \subseteq$ $A$ não enumerável que possui um certo $x$ tal que, para todos $y, z \in X$ distintos, temos $y \cap z=x$. Neste caso, $x$ recebe o nome de raiz de $X$.

Prova. Será omitida; pode ser encontrada em Jech (2006).

Prova de 2.3.6. Seja $P \subseteq F n(I, J)$ não-enumerável; como $J$ é enumerável, $\{\operatorname{dom}(p): p \in$ $P\}$ é não-enumerável (pois caso contrário $P$ seria enumerável). Pelo lema dos $\Delta$-sistemas existe $X \subseteq\{\operatorname{dom}(p): p \in P\}$ não-enumerável com uma raiz $x$. Seja então $Y=\{p \in P$ : $\operatorname{dom}(p) \in X\}$; novamente, como $J$ é enumerável, existe uma quantidade não-enumerável de elementos $p \in Y$, e estes são iguais se restringidos a $x$. Mas se $p$ e $q$ são funções assim, então são funções compatíveis, e portanto $P$ não pode ser uma anticadeia.

Lema 2.3.8 Se $C \in M$ é uma noção de forcing onde todas as suas anticadeias que estão em $M$ são enumeráveis, $G \subseteq C$ for genérico sobre $M, A, B \in M$ e $f^{G}: A \rightarrow B \in M[G]$, então existe $F: A \rightarrow P^{M}(B) \in M$ tal que $\forall a \in A\left(f^{G} a \in F a\right)$ e $\forall a \in A(\operatorname{Card}(F a) \leq \omega)^{M}$.

Prova. Seja $p \in G$ tal que $p \Vdash F u n(f) \wedge \operatorname{dom}(f)=\hat{A} \wedge \operatorname{Im}(f) \subseteq \hat{B}$. Defina

$$
F(a)=\{b \in B: \exists q \leq p(q \Vdash f(\hat{a})=\hat{b})\} .
$$

Então $F \in M$. Seja $a \in A$; para ver que $f^{G}(a) \in F(a)$, seja $b=f^{G}(a)$. Então $\exists r \in G(r \Vdash$ $f(\hat{a})=\hat{b})$; como $r, p \in G$, existe $q \in G, q \leq r, p$ e assim $q \Vdash f(\hat{a})=\hat{b}$, donde $b \in F(a)$.

Para ver que $(\operatorname{Card}(F a) \leq \omega)^{M}$, pelo axioma da escolha seja $Q \in M$ tal que $Q: F(a) \rightarrow$ $C$ e, para $b \leq F(a), Q(b) \leq p$ e $Q(b) \Vdash f(\hat{a})=\hat{b}$. Se $b, b^{\prime} \in F(a)$ e $b \neq b^{\prime}$, então $Q(b)$ é incompatível com $Q\left(b^{\prime}\right)$, pois caso contrário existiria um genérico $G^{\prime}$ contendo ambos, e aí teríamos $f^{G^{\prime}}: A \rightarrow B$ e $f^{G^{\prime}}(a)=b=b^{\prime}$, um absurdo. Logo, $\{Q(b): b \in F(a)\}$ é uma anticadeia em $C$ e, como $Q \in M$ e toda anticadeia de $C$ que está em $M$ é enumerável, temos $(\operatorname{Card}(F a) \leq \omega)^{M}$. 
Definição 2.3.9 (a) A cofinalidade de um ordinal $\alpha$ é o menor ordinal $\beta$ para o qual existe uma função $f: \beta \rightarrow \alpha$ estritamente crescente cuja imagem é ilimitada em $\alpha$. Neste caso dizemos que $f$ é cofinal em $\beta$ e escrevemos cf $(\alpha)=\beta$.

(b) Um cardinal $\kappa$ é regular se $c f(\kappa)=\kappa$, e singular se $c f(\kappa)<\kappa$.

Lema 2.3.10 $c f^{M[G]}(\alpha) \leq c f^{M}(\alpha)$.

Prova. Como $M \subseteq M[G]$, se existe $f: \beta \rightarrow \alpha, f \in M$, como " $x$ é função" é absoluto, $f \in M[G]$ e é função em $M[G]$.

Lema 2.3.11 Todo cardinal em $M[G]$ é cardinal em $M$.

Prova. Como $M$ não tem mais funções que $M[G]$, não pode existir uma bijeção entre ordinais de $M$ que não seja também uma bijeção entre os mesmos ordinais em $M[G]$.

Lema 2.3.12 Suponha que $C \in M$ e que sempre que $G \subseteq C$ é genérico sobre $M$ e que ( $\kappa$ é um cardinal regular não enumerável $)^{M}$, temos $(\kappa \text { é regular })^{M[G]}$. Então, se $\gamma$ é ordinal limite em $M$,

$$
c f(\gamma)^{M}=c f(\gamma)^{M[G]}
$$

Além disso, se $\beta \in O N^{M}$ e $\beta>\omega$, então $(\beta \text { é cardinal })^{M} \rightarrow(\beta \text { é cardinal })^{M[G]}$.

Prova. Seja $\gamma$ um ordinal limite em $M$ e $(\kappa=c f(\gamma))^{M}$. Então existe $f \in M$ tal que $f: \kappa \rightarrow \gamma$ estritamente crescente com imagem ilimitada em $\gamma$. Como $\left(\kappa\right.$ é regular) ${ }^{M}$ (pois $c f(c f(\alpha))=c f(\alpha)$ para todo $\alpha)$, temos $(\kappa \text { é regular })^{M[G]}$ pela hipótese (e se $\kappa=\omega$, usamos que $\omega$ é absoluto). Como $f \in M[G],(\kappa=c f(\gamma))^{M[G]}$.

Agora, se $\alpha \geq \omega$ é um cardinal regular de $M$, então $c f(\alpha)^{M[G]}=c f(\alpha)^{M}=\alpha$, e assim $\alpha$ é um cardinal regular em $M[G]$. Se $\beta \in O N^{M}$ e é um cardinal limite em $M$, então os cardinais regulares de $M$ são ilimitados em $\beta$; como eles permanecem ilimitados em $M[G]$, $\beta$ é cardinal limite em $M[G]$ também. Como todo cardinal infinito é regular ou limite, todo cardinal infinito de $M$ é um cardinal de $M[G]$.

Lema 2.3.13 Se $C \in M$ e toda anticadeia de $C$ que está em $M$ é enumerável, então para todo $\gamma$ limite de $M$ temos $c f(\gamma)^{M}=c f(\gamma)^{M[G]}$.

Prova. Se não, por 2.3.12 existe $\kappa \in M, \kappa>\omega,(\kappa \text { é regular })^{M}$ e $(\kappa \text { não é regular })^{M[G]}$. Assim, existe $\alpha<\kappa$ e $f^{G} \in M[G]$ tal que $f: \alpha \rightarrow \kappa$ é estritamente crescente e ilimitada. Por 2.3.8, seja $F \in M, F: \alpha \rightarrow P^{M}(\kappa)$ tal que $\forall \xi<\alpha\left(f^{G}(\xi) \in F(\xi)\right)$ e $\forall \xi<\alpha(\operatorname{Card}(F \xi) \leq \omega)^{M}$, e seja também $S=\bigcup_{\xi<\alpha} F(\xi)$. Então $S \in M$ e $S$ é um subconjunto ilimitado de $\kappa$. Aplicando em $M$ o fato de que a união de $\operatorname{Card}(\alpha)$ conjuntos enumeráveis tem cardinalidade $\operatorname{Card}(\alpha)$, temos $(\operatorname{Card}(S)=\operatorname{Card}(\alpha)<\kappa)^{M}$, contradizendo a hipótese que $(\kappa \text { é regular })^{M}$.

Lema 2.3.14 $Z F 2+\neg H C$ é consistente.

Prova. Seja $M$ um $\in$-modelo transitivo de $Z F 2$ e $C=F n\left(\omega_{2}^{M} \times \omega, 2\right) \in M$. Então toda anticadeia de $C$ que está em $M$ é enumerável (por 2.3.6) e assim $\omega_{2}^{M}=\omega_{2}^{M[G]}$. Segue de 2.3.4 que $\left(\omega_{2} \leq 2^{\omega}\right)^{M[G]}$. 


\subsection{Importando resultados de consistência com ZFC para consistência com ZF2}

Como o método usado para provar a consistência de $Z F 2+\neg H C$ é, na prática, o mesmo usado para provar a consistência de $Z F C+\neg H C$, podemos nos perguntar se não existe um método geral por trás disto. Na verdade, há um, mas para que possamos estabelecêlo será necessário primeiramente nos livrarmos da hipótese de existência de um $\in$-modelo transitivo de $Z F 2$. Para isso, vamos provar um teorema de reflexão para $Z F 2$ com semântica de Henkin.

Ao longo desta seção, usaremos a seguinte notação: se $P$ for um símbolo de predicado $n$-ário de segunda ordem, então $P \uparrow M=\left\{x \in M^{n}: P(x)\right\}$ e, de maneira semelhante para símbolos $n$-ários $p$ de função, $p\left\lceil M=\left\{\langle x, y\rangle \in M^{n+1}: p(x)=y\right\}\right.$.

Lema 2.4.1 Seja $\varphi$ uma fórmula de primeira ordem no vocabulário $\{\in\}$ tal que $V L(\varphi) \subseteq$ $\left\{x_{1}, \ldots, x_{n}, X_{1}, \ldots, X_{m}, f_{1}, \ldots f_{k}\right\}$. Então existe uma $\in$-pré-estrutura $M$ de Henkin transitiva enumerável tal que

(1) para todos $a_{2}, \ldots, a_{n} \in M$, para todos os simbolos de predicado $P_{1}, \ldots, P_{m}$ tais que $P_{1} \uparrow M, \ldots, P_{m} \uparrow M \in R^{H}$ e para todos os símbolos de função $p_{1}, \ldots, p_{k}$ tais que $p_{1} \uparrow$ $M, \ldots, p_{k} \uparrow M \in F^{H}$,

$$
\begin{gathered}
s e \exists x \varphi\left(x, a_{2}, \ldots, a_{n}, P_{1}, \ldots, P_{m}, p_{1}, \ldots, p_{k}\right)^{5}, \text { ent } \tilde{a} o \\
(\exists x \in M) \varphi^{M}\left(x, a_{2}, \ldots, a_{n}, P_{1} \uparrow M, \ldots, P_{m} \uparrow M, p_{1} \uparrow M, \ldots, p_{k} \uparrow M\right) .
\end{gathered}
$$

(2) para todos $a_{1}, \ldots, a_{n} \in M$, para todos os simbolos de predicado $P_{2}, \ldots, P_{m}$ tais que $P_{2}\left\lceil M, \ldots, P_{m} \uparrow M \in R^{H}\right.$ e para todos os símbolos de função $p_{1}, \ldots, p_{k}$ tais que $p_{1} \uparrow$ $M, \ldots, p_{k} \uparrow M \in F^{H}$,

$$
\begin{gathered}
s e \exists X \varphi\left(a_{1}, \ldots, a_{n}, X, P_{2}, \ldots, P_{m}, p_{1}, \ldots, p_{k}\right), \text { então } \\
\left(\exists X \in R^{H}\right) \varphi^{M}\left(a_{1}, \ldots, a_{n}, X, P_{2} \uparrow M, \ldots, P_{m} \uparrow M, p_{1}\left\lceil M, \ldots, p_{k} \uparrow M\right) .\right.
\end{gathered}
$$

(3) para todos $a_{1}, \ldots, a_{n} \in M$, para todos os simbolos de predicado $P_{1}, \ldots, P_{m}$ tais que $P_{1} \uparrow M, \ldots, P_{m} \uparrow M \in R^{H}$ e para todos os símbolos de função $p_{2}, \ldots, p_{k}$ tais que $p_{2} \uparrow$ $M, \ldots, p_{k} \uparrow M \in F^{H}$,

$$
\begin{gathered}
\operatorname{se} \exists f \varphi\left(a_{1}, \ldots, a_{n}, P_{1}, \ldots, P_{m}, f, p_{2}, \ldots, p_{k}\right), \text { ent } \tilde{a} o \\
\left(\exists f \in F^{H}\right) \varphi^{M}\left(a_{1}, \ldots, a_{n}, P_{1}\left\lceil M, \ldots, P_{m} \uparrow M, f, p_{2}\left\lceil M, \ldots, p_{k} \uparrow M\right) .\right.\right.
\end{gathered}
$$

Prova.Defina a função $\mu r(x)$ dada por:

$$
\mu r(x)=\{y \in x: \forall z \in x(\operatorname{rank}(y) \leq \operatorname{rank}(z))\} .
$$

Vamos supor sem perda de generalidade que $n=m=k=1$. Defina então para todos os conjuntos a, todas as classes $P$, todos os funcionais $p$ e todas as pré-estruturas $M$,

$$
\begin{gathered}
H(P, p)=\mu r\{x: \varphi(x, P, p)\} ; \\
G_{P}(M, a, p)=\mu r\{P \uparrow M: \varphi(a, P, p)\} ; \\
G_{p}(M, a, P)=\mu r\{p\lceil M: \varphi(a, P, p)\},
\end{gathered}
$$

\footnotetext{
${ }^{5}$ Onde estamos fazendo o uso comum de tomar elementos como seus próprios nomes.
} 
e sejam $|M|_{0}=\left(F^{H}\right)_{0}=\left(R^{H}\right)_{0}=\{\emptyset\}$, e defina por indução

$$
\begin{aligned}
|M|_{i+1}= & c l\left(| M | _ { i } \cup \bigcup \left\{H(P, p): P \text { é um símbolo de predicado com } P \uparrow|M|_{i} \in\left(R^{H}\right)_{i}\right.\right. \text { e } \\
& \left.p \text { é um símbolo de função com } p \uparrow|M|_{i} \in\left(F^{H}\right)_{i}\right\} \cup \\
& \left.\bigcup\left\{\operatorname{Im}\left(p \uparrow|M|_{i}\right): p \text { é um símbolo de função e } p \uparrow|M|_{i} \in\left(F^{H}\right)_{i}\right\}\right)
\end{aligned}
$$

(onde $\operatorname{cl}(X)$ é o menor conjunto transitivo que contém $X$ ). Sejam também

$$
\begin{gathered}
\operatorname{Ext}\left(\left(F^{H}\right)_{i},|M|_{i}\right)=\left\{p \uparrow|M|_{i+1}: p \text { é símbolo de função e } p \uparrow|M|_{i} \in\left(F^{H}\right)_{i}\right\}, \\
\operatorname{Ext}\left(\left(R^{H}\right)_{i},|M|_{i}\right)=\left\{P \uparrow|M|_{i+1}: P \text { é símbolo de predicado e } P \uparrow|M|_{i} \in\left(R^{H}\right)_{i}\right\},
\end{gathered}
$$

e ainda por indução

$$
\begin{aligned}
& \left(R^{H}\right)_{i+1}=\operatorname{Ext}\left(\left(R^{H}\right)_{i},|M|_{i}\right) \cup \bigcup\left\{G_{P}\left(|M|_{i}, a, p\right): a \in|M|_{i}, p \uparrow|M|_{i} \in\left(F^{H}\right)_{i}\right\} \\
& \left(F^{H}\right)_{i+1}=\operatorname{Ext}\left(\left(F^{H}\right)_{i},|M|_{i}\right) \cup \bigcup\left\{G_{p}\left(|M|_{i}, a, P\right): a \in|M|_{i}, P \uparrow|M|_{i} \in\left(R^{H}\right)_{i}\right\}
\end{aligned}
$$

e sejam

$$
\begin{gathered}
|M|=\bigcup_{i<\omega}|M|_{i}, \\
R^{H}=\left\{P \uparrow M: \exists i \in \omega\left(P \uparrow|M|_{i} \in\left(R^{H}\right)_{i}\right\} \mathrm{e}\right. \\
F^{H}=\left\{p \uparrow M: \exists i \in \omega\left(p \uparrow|M|_{i} \in\left(F^{H}\right)_{i}\right\} .\right.
\end{gathered}
$$

Vamos primeiro mostrar que $M=\left\langle|M|, R^{H}, F^{H}, \in\right\rangle$ é uma $\in$-pré-estrutura de Henkin transitiva (falta ver que é fechada pelas funções em $F^{H}$ ), e que satisfaz as propriedades (1), (2) e (3), e depois que $M$ pode ser tomada enumerável.

Defina $\bar{t}$, um conjunto definido a partir de um termo $t$ construído apenas com variáveis, digamos $x_{1}, \ldots, x_{n}, f_{1}, \ldots, f_{k}$, da seguinte forma: se $a_{1}, \ldots, a_{n}$ são conjuntos e $p_{1}, \ldots, p_{k}$ são funcionais, então definimos $\bar{t}$ por indução: se $t$ é $x_{i}$, então $\bar{t}=a_{i}$ e, se $t$ é $f_{i}\left(t_{1}, \ldots, t_{l}\right)$, então $\bar{t}=p_{i}\left(\bar{t}_{1}, \ldots, \bar{t}_{l}\right)$. Neste caso,

Lema. Se $a_{1}, \ldots, a_{n} \in M$ e $p_{1} \uparrow M, \ldots, p_{k} \uparrow M \in F^{H}$, então $\bar{t} \in M$.

Prova. Indução em $t$. Se $t$ é uma variável, é claro. Se $t$ é $f_{i}\left(t_{1}, \ldots, t_{l}\right)$, então $\bar{t}=p_{i}\left(\bar{t}_{1}, \ldots, \bar{t}_{l}\right)$, e por hipótese de indução existe $j \in \omega$ tal que $\overline{t_{1}}, \ldots, \bar{t}_{l} \in|M|_{j}$. Como $p_{i} \uparrow M \in F^{H}$, existe $j^{\prime} \in \omega$ tal que $p_{i} \uparrow|M|_{j^{\prime}} \in\left(F^{H}\right)_{j^{\prime}}$ e existe $j^{\prime \prime} \geq j, j^{\prime}$ tal que $p_{i} \uparrow|M|_{j^{\prime \prime}} \in\left(F^{H}\right)_{j^{\prime \prime}}$, pela definição da sequencia $\left(F^{H}\right)_{0},\left(F^{H}\right)_{1}, \ldots$ Logo, temos $\operatorname{Im}\left(p_{i} \uparrow|M|_{j^{\prime \prime}}\right) \subseteq|M|_{j^{\prime \prime}+1} \subseteq|M|$.

Segue então que $M$ é de fato uma $\in$-pré-estrutura de Henkin. A prova de que (1) se verifica é semelhante à prova de que (2) e (3) se verificam, e assim vamos provar apenas (1). Suponha que $\exists x \varphi(x, P, p)$ para um certo símbolo de predicado $P \operatorname{com} P \uparrow M \in R^{H}$ e para um símbolo de função $p \operatorname{com} p\left\lceil M \in F^{H}\right.$. Então, por definição de $H$, existe $x \in H(P, p)$ tal que $\varphi(x, P, p)$. Agora, como $P \uparrow M \in R^{H}$ e $p\left\lceil M \in F^{H}\right.$, por construção de $R^{H}$ e $F^{H}$, existe $i \in \omega$ tal que $P \uparrow|M|_{i} \in\left(R^{H}\right)_{i}$ e $p \uparrow|M|_{i} \in\left(F^{H}\right)_{i}$, donde $x \in|M|_{i+1}$, e assim existe $x \in M$ tal que $\varphi(x, P, p)$. Agora, para ver que $\varphi^{M}(a, P \uparrow M, p \uparrow M)$, vamos proceder por indução na complexidade de $\varphi$ :

se $\varphi$ é atômica: se $\varphi$ é $t \in s$, então $\varphi(a, P, p)$ é $\bar{t} \in \bar{s}$, que é o mesmo que $\varphi^{M}(a, P \uparrow$ $M, \overline{p\lceil M) \text {. Se } \varphi}$ é $X\left(t_{1}, \ldots, t_{l}\right)$, então $\varphi(a, P, p)$ é $P\left(\bar{t}_{1}, \ldots, \bar{t}_{l}\right)$, e $\varphi^{M}(a, P \uparrow M, p \uparrow M)$ é $\left\langle\bar{t}_{1}, \ldots, \bar{t}_{l}\right\rangle \in P \uparrow M$. Neste caso, sabemos que existe $j \in \omega$ tal que $\bar{t}_{1}, \ldots, \bar{t}_{l} \in|M|_{j}$, e que também existe $j^{\prime}>j$ tal que $P \uparrow|M|_{j^{\prime}} \in\left(R^{H}\right)_{j^{\prime}}$, donde segue o que queremos.

se $\varphi$ é $\psi \wedge \chi$ ou $\neg \psi$ ou envolve quantificadores: estes casos seguem imediatamente da hipótese de indução.

Para ver que $M$ pode ser tomada enumerável, pelo axioma da escolha seja $e$ uma função em $P(|M|)$ tal que $x \neq \emptyset \rightarrow e(x) \in x$. Defina então para cada $a \in M$, cada classe $P$ tal que $P \uparrow M \in R^{H}$ e funcional $p$ tal que $p \uparrow M \in F^{H}$, 


$$
\begin{gathered}
h(a, P, p)=e(H(a, P, p)) ; \\
g_{p}(M, a, p)=e\left(G_{p}(M, a, P)\right) ; \\
g_{P}(M, a, P)=e\left(G_{P}(M, a, p)\right) ;
\end{gathered}
$$

Tome agora $\left|M^{\prime}\right|_{0}=\left(R^{H}\right)_{0}^{\prime}=\left(F^{H}\right)_{0}^{\prime}=\{\emptyset\} \mathrm{e}$

$$
\begin{aligned}
\left|M^{\prime}\right|_{i+1}= & c l\left(| M ^ { \prime } | _ { i } \cup \left\{h(P, p): P \text { é um símbolo de predicado com } P \uparrow\left|M^{\prime}\right|_{i} \in\left(R^{H}\right)_{i}^{\prime} \mathrm{e}\right.\right. \\
& \left.p \text { é um símbolo de função com } p \uparrow\left|M^{\prime}\right|_{i} \in\left(F^{H}\right)_{i}^{\prime}\right\} \cup \\
& \left.\bigcup\left\{\operatorname{Im}\left(p \uparrow\left|M^{\prime}\right|_{i}\right): p \text { é um funcional e } p \uparrow\left|M^{\prime}\right|_{i} \in\left(F^{H}\right)_{i}^{\prime}\right\}\right) . \\
\left(R^{H}\right)_{i+1}^{\prime}= & \operatorname{Ext}\left(\left(R^{H}\right)_{i}^{\prime},\left|M^{\prime}\right|_{i}\right) \cup\left\{g_{P}\left(\left|M^{\prime}\right|_{i}, a, p\right): a \in\left|M^{\prime}\right|_{i}, p \uparrow\left|M^{\prime}\right|_{i} \in\left(F^{H}\right)_{i}^{\prime}\right\} \\
\left(F^{H}\right)_{i+1}^{\prime}= & \operatorname{Ext}\left(\left(F^{H}\right)_{i}^{\prime},\left|M^{\prime}\right|_{i}\right) \cup\left\{g_{p}\left(\left|M^{\prime}\right|_{i}, a, P\right): a \in\left|M^{\prime}\right|_{i}, P \uparrow\left|M^{\prime}\right|_{i} \in\left(R^{H}\right)_{i}^{\prime}\right\}
\end{aligned}
$$

A $\in$-pré-estrutura $M^{\prime}$ formada assim satisfaz (1), (2) e (3) do mesmo modo que $M$ mas com a diferença de ser enumerável.

Corolário 2.4.2 Se $\varphi_{1}, \ldots, \varphi_{l}$ são $\in$-fórmulas tais que $V L\left(\varphi_{i}\right) \subseteq\left\{x_{1}, \ldots, x_{n}, X_{1}, \ldots, X_{m}\right.$, $\left.f_{1}, \ldots f_{k}\right\}, 1 \leq i \leq l$, então existe uma $\in$-pré-estrutura de Henkin transitiva enumerável $M$ que satisfaz as propriedades (1), (2) e (3) do lema 2.4 .1 para $\varphi_{1}, \ldots, \varphi_{l}$.

Prova. Basta tomar $\varphi_{1} \wedge \ldots \wedge \varphi_{l}$ no lugar de $\varphi$ na prova de 2.4.1.

Teorema 2.4.3 Seja $\varphi$ uma $\in$-fórmula tal que $V L(\varphi) \subseteq\left\{x_{1}, \ldots, x_{n}, X_{1}, \ldots, X_{m}, f_{1}, \ldots f_{k}\right\}$. Então existe uma $\in$-pré-estrutra de Henkin $M$ transitiva enumerável tal que para todos $a_{1}, \ldots, a_{n} \in M$, todas as classes $P_{1}, \ldots, P_{m}$ tais que $P_{1} \uparrow M, \ldots, P_{m} \uparrow M \in R^{H}$ e todos os funcionais $p_{1}, \ldots, p_{k}$ tais que $p_{1} \uparrow M, \ldots, p_{k} \uparrow M \in F^{H}$ temos

$$
\begin{gathered}
\varphi\left(a_{1}, \ldots, a_{n}, P_{1}, \ldots, P_{m}, p_{1}, \ldots, P_{k}\right) \leftrightarrow \\
\varphi^{M}\left(a_{1}, \ldots, a_{n}, P_{1}\left\lceil M, \ldots, P_{m} \uparrow M, p_{1} \uparrow M, \ldots, p_{k}\lceil M) .\right.\right.
\end{gathered}
$$

Prova. Vamos supor que $n=m=k=1$ e que $\varphi_{1}, \ldots, \varphi_{l}$ são todas as subfórmulas de $\varphi$. Pelo lema 2.4.1, existe uma $\in$-pré-estrutura $M$ tal que, para $j=1, \ldots, l$,

$$
\begin{gathered}
\exists x \varphi_{j}(x, P, p) \rightarrow(\exists x \in M) \varphi_{j}^{M}(x, P \uparrow M, p \uparrow M), \\
\exists X \varphi_{j}(a, X, p) \rightarrow\left(\exists X \in R^{H}\right) \varphi_{j}^{M}(a, X, p \uparrow M), \\
\exists f \varphi_{j}(a, P, f) \rightarrow\left(\exists f \in F^{H}\right) \varphi_{j}^{M}(a, P \uparrow M, f),
\end{gathered}
$$

para todos $a \in M$, todas as classes $P$ com $P \uparrow M \in R^{H}$ e todos os funcionais $p$ com $p \uparrow M \in F^{H}$. Agora, os casos em que $\varphi$ é atômica ou é $\psi \wedge \chi$ ou $\neg \psi$ seguem imediatamente da hipótese de indução, e se $\varphi$ for da forma $\exists x \psi$, com $x$ de primeira ou segunda ordem, então o resultado segue de 2.4.1.

Corolário 2.4.4 Se $Z F 2 \vdash \exists C(C$ é uma noção de forcing) e $Z F 2 \vdash \exists p \in C(p \Vdash \varphi)$, então $\operatorname{Con}(Z F 2) \rightarrow \operatorname{Con}(Z F 2+\varphi)$.

Prova. Suponha que $Z F 2+\varphi$ seja inconsistente e que $\varphi_{1}, \ldots, \varphi_{n}$ é uma prova de $\psi \wedge \neg \psi$ a partir de $Z F 2+\varphi$, e suponha ainda que os axiomas usados nesta prova estejam no começo da prova; digamos que sejam $\varphi_{1}, \ldots, \varphi_{m}$, e que $\varphi_{m}$ seja $\varphi$, de tal forma que $\varphi_{1}, \ldots, \varphi_{m-1}$ são todos axiomas de $Z F 2$ (ou do sistema $D 2+$ ). Então, pelo teorema 2.4.3, existe um $\in-$ modelo transitivo enumerável $M$ de $\varphi_{1}, \ldots, \varphi_{m-1}$ e ainda de todos os axiomas necessários para provar a existência de $C$ e de $p$. Então, existe um genérico $G$ sobre $M$ tal que $M[G] \vDash$ $\varphi_{1} \wedge \ldots \wedge \varphi_{m} ;$ mas daí $M[G] \vDash \psi \wedge \neg \psi$, um absurdo.

Corolário 2.4.5 Suponha que podemos provar pelo método de forcing em $Z F C$ que $Z F C+\varphi$ é consistente. Então $Z F 2+\varphi$ é consistente.

Prova. Se $Z F C$ prova que $C$ é uma noção de forcing e que existe $p \in C$ tal que $p \Vdash \varphi$, então $Z F 2$ também prova a existência de $C$ e que $p \Vdash \varphi$. 


\subsection{Forcing para Classes}

Como classes próprias são entendidas intuitivamente como conjuntos "grandes", surge a natural questão de considerar se é possível que tenhamos um conjunto de condições que seja uma classe, conseguindo desta forma um genérico $G$ que seja também uma classe. A resposta é que sim, é possível, mas vamos precisar exigir algo a mais sobre o genérico $G$; os genéricos que satisfazerem a condição desejada serão chamados de fortemente genéricos, seguindo Chuaqui (1972).

A questão de se $G$ ser fortemente genérico é uma condição necessária para se provar que $M[G]$ é um modelo de $Z F 2$ não é abordada em Chuaqui (1972), mas nós esperamos poder fornecer uma motivação técnica. Durante toda a prova de que $M[G]$ satisfazia $Z F 2$, no caso em que $C$ era um conjunto de $M$, foi comum definirmos conjuntos do tipo $c=\{\langle x, p\rangle \in$ $\operatorname{dom}(a) \times C: \ldots\}$ que era por sua vez levado em $M[G]$ pela função $c \mapsto c^{G}$. O problema agora é que não temos $C \in M$, e portanto não é possível formar o conjunto $c$ em $M$. Uma primeira ideia então pode ser aproximar a classe $C$ por conjuntos $C^{\prime}$ em $M$, tendo algo do tipo $C=\bigcup\left\{C^{\prime}: C^{\prime}\right.$ aproxima $\left.C\right\}$. Resta saber como podemos definir essas aproximações para que sejam úteis na construção de $c$.

Se $c^{G}$ tiver tamanho, digamos, $\alpha$, então $C^{\prime}$ precisará "ter a informação" de $\alpha$ densos da forma $\{p: p \Vdash x \in c \vee p \Vdash \neg(x \in c)\}$, um para cada $x^{G} \in c^{G}$. Podemos tentar exigir então que $C^{\prime}$, que vamos agora chamar de $C(\alpha)$, tenha esta "quantidade de informação" necessária para a definição de $c^{G}$. Seria algo do tipo:

$$
\begin{gathered}
\forall \alpha \in O N^{M} \exists C(\alpha) \in M\left(C(\alpha) \subseteq C \wedge \forall\left\langle D_{\beta}: \beta<\alpha\right\rangle \in M \text { onde cada } D_{\beta}\right. \text { é denso temos } \\
\left.\forall \beta<\alpha \rightarrow \exists p \in C(\alpha) \cap G \cap D_{\beta}\right) .
\end{gathered}
$$

No entanto, essa definição parece muito exigente: os conjuntos $C(\alpha)$ agora vão precisar intersectar conjuntos densos arbitrários, e assim podem ficar grandes demais para serem conjuntos. Mas, reparemos que, como $G$ é denso, $G$ já intersecta os densos $D_{\beta}$. Então poderíamos exigir um pouco menos na segunda linha; talvez

$$
\forall \beta<\alpha \exists p \in C(\alpha) \wedge \exists q \in D_{\beta} \cap G(q \leq p) .
$$

Mas neste caso retornamos ao problema inicial: o $q \in D_{\beta} \cap G$ não está em $C(\alpha)$, o que seria necessário para nossos métodos de construção de $c$. Surge então a ideia de tomar um $p \in C(\alpha) \cap G$ e, como $\exists q \in G \cap D_{\beta}$ (pois $G$ é genérico), tomaríamos $p \wedge q$ como a condição "correta" tal que de $\langle x, p\rangle \in c$ e $p \wedge q \Vdash x \in c$, teríamos $x^{G} \in c^{G}$. Assim, a segunda linha fica

$$
\forall \beta<\alpha \rightarrow \exists p \in C(\alpha) \cap G \exists q \in G \cap D_{\beta} \exists p \wedge q .
$$

Contudo, há ainda mais um problema. Suponha que tomássemos o seguinte $c$ :

$$
c=\left\{\langle x, p\rangle \in \operatorname{dom}(a) \times C^{\prime}: \exists q \in D(p \wedge q \Vdash A(x))\right\},
$$

semelhante ao caso do lema 2.2.14, onde $c^{G}=A^{G}$ e $A^{G} \subseteq a^{G}$. Para mostrarmos que $c^{G} \subseteq A^{G}$, tome $x^{G} \in c^{G}$. Então $x \in \operatorname{dom}(a)$ e temos um $p \in C^{\prime}$ tal que $\langle x, p\rangle \in c$ e existe $q \in D$ tal que

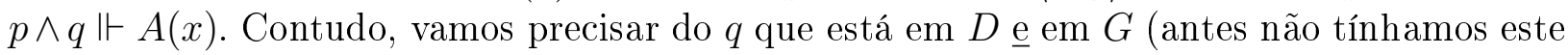
problema pois $G$ estava todo dentro de $C^{\prime}$ ). Podemos pensar assim: queremos $q \in D \cap G$, mas o $q$ está sendo fornecido pela definição de $c$, e $c \in M$; como $G \notin M$, fica difícil conseguir que $q \in G$. Uma solução então pode ser a seguinte: $q$ não é dado por $c$, isto é, ao construirmos $c$, já saberíamos de antemão da existência de um $q \in G$ com a propriedade desejada. Neste caso, chegamos à definição procurada: 
Definição 2.5.1 Se $C \in R^{H}$ é uma classe parcialmente ordenada de $M$, então $G \subseteq C$ é fortemente genérico sobre $M$ se for genérico sobre $M$ e se

$$
\begin{aligned}
\forall \alpha \in O N^{M} \exists C(\alpha) \in & M\left(C(\alpha) \subseteq C \wedge \forall\left\langle D_{\beta}: \beta<\alpha\right\rangle \in M \text { onde cada } D_{\beta}\right. \text { é denso temos } \\
& \left.\exists q \in G \forall \beta<\alpha \exists p \in C(\alpha) \cap G\left(\exists p \wedge q \in D_{\beta}\right)\right) .
\end{aligned}
$$

Observe que se $C \in M$, então todo genérico $G \subseteq C$ é fortemente genérico; isso não é de se surpreender, já que a definição de fortemente genérico foi montada de tal forma que ela aproximasse o "trabalho" de $C$ no caso em que $C \in M$. Essa alteração não afeta a noção de forcing, de tal forma que as definições de $M[G]$ (2.2.5) e de forcing (2.2.6) continuarão as mesmas. Assim, teoremas baseados nessas definições, como os lemas 2.2.7, 2.2.8 e o teorema 2.2.9 continuarão válidos. A prova de que existe um genérico (2.2.4) também pode ser facilmente adaptada para o caso de classes; mais tarde vamos nos preocupar com a existência de fortemente genéricos. Veja também que na prova de que $M[G]$ é um modelo de Henkin correto para $D 2+$ (formada pelos lemas 2.2.12 e 2.2.13) não usamos em nenhum momento o fato de que $C \in M$, donde ela permanece válida no nosso caso. Na verdade, só nos resta mostrar que $M[G]$ é um modelo de $Z F 2$.

Uma observação final: a definição de Chuaqui (1972) de fortemente genérico difere um pouco da nossa: considere a seguinte definição:

Definição 2.5.2 D é uma $C-$ seção $s e \forall p(p \in D \rightarrow \forall q(q \leq p \rightarrow q \in D))$.

Em Chuaqui (1972) temos a definição menos exigente de que as sequencias $\left\langle D_{\beta}: \beta<\alpha\right\rangle$ sejam seções densas. Não utilizamos esta definição aqui pois a necessidade de que tomemos apenas seções densas só se mostrará na próxima seção.

Teorema 2.5.3 Sejam $M$ um $\in$-modelo transitivo enumerável de ZF2 correto para D2+, $C \in R^{H}$ uma noção de forcing em $M e G \subseteq C$ fortemente genérico sobre $M$. Então $M[G]$ é um $\in-$ modelo transitivo enumerável de ZF2 correto para D2+ com os mesmos ordinais que $M, M \subseteq M[G]$ e $G \in M[G]$. Além disso, é o menor $\in$-modelo transitivo com tais propriedades.

Prova. Só precisamos mostrar que $M[G]$ satisfaz os axiomas das partes e da substituição, pois a prova de todo o resto é igual ao caso em que $C \in M$. A prova se fará em alguns lemas, análogos aos usados para a prova do teorema 2.2.11.

Lema 2.5.4 Se $a^{G} \in M[G]$, então existe $C^{\prime} \in M$ tal que para todo $A^{G} \in R[G]^{H}$ unário tal que $A^{G} \subseteq a^{G}$, existe $c \in M$ tal que $c \subseteq \operatorname{dom}(a) \times C^{\prime}$ e $A^{G}=c^{G}$.

Prova. Seja $\left\langle x_{\beta}: \beta<\alpha\right\rangle \in M$ uma enumeração de $\operatorname{dom}(a)$. Dado $A^{G} \in R[G]^{H}$, considere a sequencia de densos:

$$
D_{\beta}=\left\{p: p \Vdash A\left(x_{\beta}\right) \vee p \Vdash \neg A\left(x_{\beta}\right)\right\} .
$$

Como $G$ é fortemente genérico, existe $C^{\prime} \in M$ e $q \in G$ tais que

$$
\forall \beta<\alpha \exists p \in C^{\prime} \cap G\left(\exists p \wedge q \in D_{\beta}\right) .
$$

Considere agora

$$
c=\left\{\langle x, p\rangle \in \operatorname{dom}(a) \times C^{\prime}: p \wedge q \Vdash A(x)\right\} .
$$


Se $x^{G} \in c^{G}$, então $\exists p \in G(\langle x, p\rangle \in c)$, donde $x \in \operatorname{dom}(a)$ e $p \wedge q \Vdash A(x)$. Como $p, q \in G$, temos $p \wedge q \in G$ e assim $x^{G} \in A^{G}$. Por outro lado, se $x^{G} \in A^{G}$, então $x^{G} \subseteq a^{G}$; neste caso $x \in \operatorname{dom}(a)$ e podemos tomar $\beta<\alpha$ tal que $x=x_{\beta}$. Logo, $\exists p \in C^{\prime} \cap G$ tal que $p \wedge q \Vdash A(x)$ ou $p \wedge q \Vdash \neg A(x)$; mas como $x^{G} \in A^{G}$, temos $p \wedge q \Vdash A(x)$, e assim $\langle x, p\rangle \in c$, donde $x^{G} \in c^{G}$.

Lema 2.5.5 Se $A^{G} \in R[G]^{H}$ é unária e $\exists b \in M\left(\forall x^{G} \in A^{G} \exists y \in b\left(y^{G}=x^{G}\right)\right)$, então

$$
\exists c^{G} \forall x^{G}\left(x^{G} \in c^{G} \leftrightarrow x^{G} \in A^{G}\right) .
$$

Prova. Igual a 2.2.15.

Lema 2.5.6 $M[G]$ satisfaz o axioma das partes.

Prova. Dado $a^{G} \in M[G]$, por compreensão de segunda ordem temos

$$
P\left(a^{G}\right) \cap M[G] \in R[G]^{H} .
$$

Agora, pelo lema 2.5.4,

$$
\exists C^{\prime} \in M \forall A^{G} \in R[G]^{H}\left(A^{G} \subseteq a^{G} \rightarrow \exists c \in M\left(c \subseteq \operatorname{dom}(a) \times C^{\prime} \wedge c^{G}=A^{G}\right)\right),
$$

o que equivale a

$$
\exists C^{\prime} \in M \forall A^{G} \in P\left(a^{G}\right) \cap R[G]^{H} \exists c \in M\left(c \in P\left(\operatorname{dom}(a) \times C^{\prime}\right) \wedge c^{G}=A^{G}\right) .
$$

Mas, se $A^{G} \in P\left(a^{G}\right) \cap M[G]^{H}$, então $A^{G} \in P\left(a^{G}\right) \cap R[G]^{H}$, por compreensão de segunda ordem. Tome então $b=P\left(\operatorname{dom}(a) \times C^{\prime}\right) \cap M$; daí $b \in M$ e usamos o lema 2.5.5.

Lema 2.5.7 $M[G]$ satisfaz o axioma da substituição de segunda ordem.

Prova. A prova é análoga a 2.2.18. Seja $f^{G} \in F[G]^{H}$ unária e $a^{G} \in M[G]$; temos que $f_{*}^{G}\left(a^{G}\right) \in R[G]^{H}$. Seja $\left\langle x_{\beta}<\alpha\right\rangle \in M$ uma enumeração de $\operatorname{dom}(a)$ e $\varphi$ a fórmula $x_{1} \in$ $x_{2} \wedge f x_{1}=x_{3}$; considere, para cada $\beta<\alpha$,

$$
D_{\beta}=\left\{p \in C: \exists w\left(p \Vdash(\exists y \varphi \rightarrow \varphi)\left(x_{\beta}, a, w\right)\right)\right\} .
$$

Como em 2.2.18, cada $D_{\beta}$ é denso. Como $G$ é fortemente genérico, existem $C^{\prime} \in M$ e $q \in G$ tais que

$$
(*) \forall \beta<\alpha \exists p \in C^{\prime} \cap G\left(\exists p \wedge q \in D_{\beta}\right) \text {. }
$$

Considere agora, para cada $x_{\beta} \in \operatorname{dom}(a)$, cada $p \in C^{\prime}$, por substituição,

$$
A_{x_{\beta}, p}=\left\{w: p \wedge q \Vdash(\exists y \varphi \rightarrow \varphi)\left(x_{\beta}, a, w\right)\right\} .
$$

Dado $\beta<\alpha$, por $(*)$, existe $p \in G \cap D_{x}$ e, assim, $A_{x_{\beta}, p} \neq \emptyset$; como $M$ satisfaz o axioma da escolha, existe

$$
g \in \prod\left\{A_{x, p}: x \in \operatorname{dom}(a) \wedge p \in C \wedge A_{x, p} \neq \emptyset\right\} .
$$

Seja $w=g\left\langle x_{\beta}, p\right\rangle$. Então $p \wedge q \Vdash(\exists y \varphi \rightarrow \varphi)(x, a, w)$ e o fim da demonstração é exatamente como em 2.2.18. 


\subsection{Cardinais em $\mathrm{M}[\mathrm{G}]$ para $\mathrm{G}$ fortemente genérico}

Vamos agora estabelecer condições para a existência de classes fortemente genéricas. Vamos exigir algumas condições sobre a noção de forcing $C$ de tal forma que toda classe $C$-genérica sobre $M$ será fortemente genérica.

Nesta seção cabe uma observação. Mencionamos no final da última seção que a definição de fortemente genérico em Chuaqui (1972) é diferente da nossa; lá exigimos que exista o $C^{\prime} \in M$ que aproxima $C$ apenas para as sequencias de seções densas. Nós vamos usar esta definição de fortemente genérico aqui. Então, apenas para ressaltar:

Definição. Se $C \in R^{H}$ é uma classe parcialmente ordenada de $M$, então $G \subseteq C$ é fortemente genérico sobre $M$ se for genérico sobre $M$ e se

$\forall \alpha \in O N^{M} \exists C^{\prime} \in M\left(C^{\prime} \subseteq C \wedge \forall\left\langle D_{\beta}: \beta<\alpha\right\rangle \in M\right.$ onde cada $D_{\beta}$ é uma seção densa temos $\left.\exists q \in G \forall \beta<\alpha \exists p \in C^{\prime} \cap G\left(\exists p \wedge q \in D_{\beta}\right)\right)$.

Definição 2.6.1 (a) A noção de forcing $\langle C, \leq\rangle$ é coerente se:

(i) Existe uma familia $\left\langle C_{\xi}: \xi \in O N\right\rangle \in F^{H}$ tal que $\alpha \leq \beta \rightarrow C_{\alpha} \subseteq C_{\beta}$ e $C=\bigcup C_{\alpha}$.

(ii) $\forall \xi \in O N \exists C^{\xi} \subseteq C \forall p \in C_{\xi} \forall q \in C^{\xi} \exists p \wedge q$ e a funçã $f: C_{\xi} \times C^{\xi} \rightarrow C$ definida por $f_{\xi}(p, q)=p \wedge q$ é um isomorfismo de $\left\langle C_{\xi} \times C^{\xi}, \leq\right\rangle$ sobre $\langle C, \leq\rangle$, onde $\langle p, q\rangle \leq\langle r, s\rangle \Leftrightarrow p \leq r$ $e q \leq s$.

(iii) Se p é compativel com $q$, então existe $p \wedge q$.

(b) Seja $\langle C, \leq\rangle$ uma noção de forcing coerente. Defina para cada $\xi \in O N, p \in C$, os elementos $(p)_{\xi}$ e $(p)^{\xi}$ pela condição

$$
f_{\xi}\left\langle(p)_{\xi},(p)^{\xi}\right\rangle=p
$$

Lema 2.6.2 Se $\langle C, \leq\rangle$ é coerente e $G$ é genérico, então $G_{\xi}=G \cap C_{\xi}$ é $C_{\xi^{-}}$genérico sobre $M$.

Prova. Como $1=(1)_{\xi} \wedge(1)^{\xi}$, temos $1 \in C_{\xi}$. Se $p \in G_{\xi}$ e $q \in C_{\xi}$, com $p \leq q$, temos $q \in G_{\xi}$ pois $q \in G$. Se $p, q \in G_{\xi}$, então $\exists r \in G(r \leq p, q)$ e $r \leq p \Leftrightarrow r=(r)_{\xi} \wedge(r)^{\xi} \leq(p)_{\xi} \wedge(p)^{\xi}=p$ e, pelo isomorfismo $C_{\xi} \times C^{\xi} \cong C$, segue que $(r) \xi \leq(p)_{\xi}$, e semelhantemente temos $(r) \xi \leq(q)_{\xi}$. Como $p \wedge 1=(p)_{\xi} \wedge(p)^{\xi}$ e $p \in C_{\xi}$, segue também do isomorfismo que $p=(p)_{\xi}$, e análogamente que $q=(q)_{\xi}$. Logo $(r)_{\xi} \leq p, q$ e $(r)_{\xi} \in G_{\xi}$.

Por último, seja $D$ um conjunto $C_{\xi}$-denso em $M$. Então como $f_{\xi}$ é isomorfismo, a imagem de $f_{\xi}$ por $D \times C^{\xi}$ é $C$-densa em $M$; tome então $q$ que esteja nesta imagem e em $G$. Então $q \leq(q)_{\xi} \in G_{\xi}$ e $(q)_{\xi} \in D$.

A partir daqui, suponha sempre que $C$ é uma noção de forcing coerente.

Definição 2.6.3 Seja $\kappa$ um cardinal, $\gamma, \xi \in O N$ e g uma sequência crescente de ordinais com dominior $\gamma$ tal que se $x_{\alpha}$ é uma outra sequencia de ordinais, então $g\left(\sup _{\beta<\alpha} x_{\alpha}\right)=$ $\sup _{\beta<\alpha} g\left(x_{\alpha}\right)$. Então

(a) C satisfaz a condição da $\kappa$-cadeia se $\forall E \subseteq C(p, q \in E \rightarrow p$ e $q$ são incompatíveis $)$ vale que $\operatorname{Card}(E)<\kappa$.

(b) $C$ é $\kappa$-fechado se $\forall E \subseteq C(E$ é linearmente ordenado e $\operatorname{Card}(E)<\kappa(\exists p \in C \forall q \in$ $E(p<q)))$.

(c) C satisfaz a condição da $\kappa, \xi$-densidade, ou é $\kappa, \xi$-denso, se para toda sequencia $\left\langle D_{\alpha}: \alpha<\gamma\right\rangle$ de seções $C-$ densas com $\gamma \leq \kappa$ temos $\forall p \in C \exists q \leq p \wedge \exists \Pi \subseteq C_{\xi}(\operatorname{Card}(\Pi) \leq$ 
$\kappa \wedge \forall \alpha<\gamma \forall q^{\prime} \leq q \exists r \in \Pi\left(r\right.$ é compativel com $q^{\prime}$ e $\left.\left.(r \wedge q) \in D_{\alpha}\right)\right)$. Se, além disso, $(q)_{\xi}=(p)_{\xi}$, então dizemos que $C$ satisfaz a condição da $\kappa, \xi$-densidade forte.

(d) $C$ satisfaz a condição da $\gamma, g$-escadaria se para toda sequencia decrescente de condiçôes $\left\langle p_{\xi}: \xi<\alpha\right\rangle$ com $\alpha<\gamma$ tal que $\left(p_{\eta}\right)_{g(\xi)}=\left(p_{\xi}\right)_{g(\xi)}$ para $\xi \leq \eta<\alpha$, existe um $r \in C$ tal que

$$
r \leq p_{\xi} e(r)_{g(\xi)}=\left(p_{\xi}\right)_{g(\xi)} \text { para todo } \xi<\alpha .
$$

Lema 2.6.4 Se $\mu \leq \xi$ e $p \in C$, então $\left((p)_{\xi}\right)_{\mu}=(p)_{\mu}$.

Prova. Temos que

$$
\text { (i) } \quad p=(p)_{\xi} \wedge(p)^{\xi}=(p)_{\mu} \wedge(p)^{\mu} \mathrm{e}(p)_{\xi}=\left((p)_{\xi}\right)_{\mu} \wedge\left((p)_{\xi}\right)^{\mu},
$$

por definição. Logo

$$
\text { (ii) } \quad p=\left[\left((p)_{\xi}\right)_{\mu} \wedge\left((p)_{\xi}\right)^{\mu}\right] \wedge(p)^{\xi}
$$

Mas

$$
(\text { iii }) \quad(p)^{\xi}=\left((p)^{\xi}\right)_{\mu} \wedge\left((p)^{\xi}\right)^{\mu},
$$

donde segue que $(p)^{\xi} \leq\left((p)^{\xi}\right)_{\mu}$. Agora, $f_{\xi}$ é bijetora, e como $\left((p)^{\xi}\right)_{\mu} \in C_{\mu} \subseteq C_{\xi}$, temos $f_{\xi}\left\langle\left((p)^{\xi}\right)_{\mu},(p)^{\xi}\right\rangle=f_{\xi}\left\langle 1,(p)^{\xi}\right\rangle$, o que implica que $\left((p)^{\xi}\right)_{\mu}=1 \mathrm{e}$, ainda por $(i i i)$,

$$
\text { (iv) }(p)^{\xi}=\left((p)^{\xi}\right)^{\mu} \text {. }
$$

Agora, como $p=(p)_{\xi} \wedge(p)^{\xi}$, temos $p \leq(p)_{\xi},(p)^{\xi}$, e assim $(p)^{\mu} \leq\left((p)_{\xi}\right)^{\mu}$ e $(p)^{\mu} \leq\left((p)^{\xi}\right)^{\mu}$. Consequentemente

$$
(v) \quad\left((p)_{\xi}\right)_{\mu} \wedge(p)^{\mu} \leq\left((p)_{\xi}\right)_{\mu} \wedge\left((p)_{\xi}\right)^{\mu} \wedge\left((p)^{\xi}\right)^{\mu}=p,
$$

por (ii) e (iii). Temos também que como $p \leq(p)_{\xi}$,

$$
\text { (vi) }(p)_{\mu} \leq\left((p)_{\xi}\right)_{\mu} \text {. }
$$

Daí $p \leq\left((p)_{\xi}\right)_{\mu} \wedge(p)^{\mu}$ por (i) e (vi). Logo, usando $(v)$ e (i), temos $p=\left((p)_{\xi}\right)_{\mu} \wedge(p)^{\mu}=$ $(p)_{\mu} \wedge(p)^{\mu}$ e daí $\left((p)_{\xi}\right)_{\mu}=(p)_{\mu}$.

Lema 2.6.5 Suponha que $C_{\xi}$ satisfaça a condição da $\kappa^{+}$-cadeia e que $C^{\xi}$ seja $\kappa^{+}$-fechado. Se $D$ é uma seção densa de $C$ e $p \in C$, então $\exists q \leq p\left((q)_{\xi}=(p)_{\xi} \wedge \exists \Pi \subseteq C_{\xi}(\operatorname{Card}(\Pi) \leq\right.$ $\kappa \wedge \forall q^{\prime} \leq q \exists r \in \Pi\left(r\right.$ é compativel com $q^{\prime}$ e $\left.\left.\left.r \wedge q \in D\right)\right)\right)$.

Prova. Pelo lema de Zorn, seja $X \subseteq D$ maximal tal que

(i) $X \neq \emptyset$

(ii) $q \in X \rightarrow q \leq p$ e

(iii) $r, s \in X \rightarrow\left((r)_{\xi}\right.$ é incompatível com $(s)_{\xi}$ e $\left((r)^{\xi} \leq(s)^{\xi}\right.$ ou $\left.\left.(s)^{\xi} \leq(r)^{\xi}\right)\right)$.

Considere $X_{\xi}=\left\{(r)_{\xi}: r \in X\right\}$ e $X^{\xi}=\left\{(r)^{\xi}: r \in X\right\}$. Como $C_{\xi}$ satisfaz a condição da $\kappa^{+}$-cadeia, temos $\operatorname{Card}\left(X_{\xi}\right)=\operatorname{Card}\left(X^{\xi}\right) \leq \kappa$; tome $\Pi=X_{\xi}$. Como $X^{\xi}$ é linearmente ordenado e $C^{\xi}$ é $\kappa^{+}$-fechado, existe $s \in C^{\xi}$ tal que $s<(t)^{\xi}$ para todo $t \in X$. Tome então $q=(p)_{\xi} \wedge s$; como $s \in C^{\xi}$, do isomorfismo $f_{\xi}$ segue que $(q)_{\xi}=(p)_{\xi}$.

Vamos mostrar agora que para todo $r \in \Pi, r \wedge q \in D$ : seja $t \in X$ tal que $r=(t)_{\xi}$. Note primeiro que $r$ é compatível com $q$, já que $q=(p)_{\xi} \wedge s<(t)_{\xi}=r$. Veja também que como $t \in X$, temos que $t \leq p$ e assim $r=(t)_{\xi} \leq(p)_{\xi}$; logo $r \wedge q=(t)_{\xi} \wedge(p)_{\xi} \wedge s=(t)_{\xi} \wedge s<$ $(t)_{\xi} \wedge(t)^{\xi}=t \in X \subseteq D$. Como $D$ é seção, $r \wedge q \in D$.

Seja agora $q^{\prime} \leq q$; vamos mostrar que podemos tomar $r \in \Pi$ que seja compatível com $q^{\prime}$. Como $D$ é seção, existe $q^{\prime \prime} \leq q^{\prime}$ em $D$. Se $\left(q^{\prime \prime}\right)_{\xi} \in \Pi$, tomamos $r=\left(q^{\prime \prime}\right)_{\xi}$; se não, como $X$ é maximal, existe $t \in X$ tal que $(t)_{\xi}$ é compatível com $\left(q^{\prime \prime}\right)_{\xi}$, e aí tomamos $r=(t)_{\xi}$. 
Lema 2.6.6 Se $C_{\xi}$ satisfaz a condição da $\kappa^{+}-$cadeia e $C^{\xi}$ é $\kappa^{+}$-fechado, então $C$ satisfaz a condição da $\kappa, \xi$-densidade forte.

Prova. Seja $\left\langle D_{\alpha}: \alpha<\beta\right\rangle, \beta \leq \kappa$ uma sequencia de $C$-seções densas e $p \in C$. Vamos definir por recursão $q_{\eta}, \Pi_{\eta}$ para $\eta<\beta$ tal que:

(i) $\eta \leq \eta^{\prime}<\beta \rightarrow q_{\eta^{\prime}} \leq q_{\eta} \leq p$

(ii) $\left(q_{\eta}\right)_{\xi}=(p)_{\xi}$,

(iii) $\Pi_{\eta} \subseteq C_{\xi}, \operatorname{Card}\left(\Pi_{\eta}\right) \leq \kappa \mathrm{e}$

(iv) $\forall q^{\prime} \leq q_{\eta} \exists r \in \Pi_{\eta}\left(r\right.$ é compatível com $q^{\prime}$ e $\left.r \wedge q_{\eta} \in D_{\eta}\right)$.

Se $\eta=\gamma+1$, aplique 2.6.5 a $q_{\gamma}, D_{\eta}$.

Se $\eta=\bigcup \eta$, como $C^{\xi}$ é $\kappa^{+}$-fechado, existe $s \in C^{\xi}$ tal que $s<\left(q_{\gamma}\right)^{\xi}$ para $\gamma<\eta$. Tome $t=(p)_{\xi} \wedge s$ e aplique 2.6.5 a $t, D_{\eta}$.

Agora, como $C^{\xi}$ é $\kappa^{+}$-fechado, tome $s \in C^{\xi}$ tal que $s<\left(q_{\eta}\right)^{\xi}$ para $\eta<\beta$, e sejam $q=(p)_{\xi} \wedge s$ e $\Pi=\bigcup_{\eta<\beta} \Pi$; então $q=(p)_{\xi} \wedge s \leq(p)_{\xi} \wedge\left(q_{\eta}\right)^{\xi} \leq(p)_{\xi} \wedge(p)^{\xi}=p$ e $\operatorname{Card}(\Pi) \leq$ $\kappa . \kappa=\kappa$. Além disso, dados $q^{\prime} \leq q$ e $\alpha<\beta$, como $q^{\prime} \leq(p)_{\xi} \wedge\left(q_{\alpha}\right)^{\xi}=\left(q_{\alpha}\right)_{\xi} \wedge\left(q_{\alpha}\right)^{\xi}=q_{\alpha}$, existe $r \in \Pi_{\alpha}$ tal que $r$ é compatível com $q^{\prime}$ e $r \wedge q_{\alpha} \in D_{\alpha}$. Mas $r \in \Pi$ e $r \wedge q \leq r \wedge q_{\alpha}$, donde $r \wedge q \in D_{\alpha}$. Como $s \in C^{\xi}$ e $q=(p)_{\xi} \wedge s$, do isomorfismo $f_{\xi}$ segue que $(q)_{\xi}=(p)_{\xi}$.

Lema 2.6.7 Sejam $H$ uma $\lambda$-sequencia crescente de cardinais, $\mu=\Sigma_{\xi<\lambda} H \xi$, g uma $\lambda-$ sequencia crescente de ordinais, $\gamma=$ limg, $C_{g \xi}$ satisfazendo a condição da $H(\xi)^{+}-$cadeia, $C^{g \xi}$ $H(\xi)^{+}$-fechado e $C$ satisfazendo a condição da $\lambda, g$-escadaria. Então $C$ satisfaz a condição da $\mu, \gamma-$ densidade.

Prova. Só precisamos provar para sequencias de seções densas de tamanho $\mu$. Seja $\left\langle D_{\alpha}\right.$ : $\alpha<\mu\rangle$ uma sequencia de seções densas. Tome $\gamma<\mu$; vamos escrever $\gamma$ como uma soma em função de $H$ e de um certo $\alpha<H(\xi)$, onde $\xi=\mu \sigma(\gamma<H \sigma)$. Se $\xi=\eta+1$, tome $\gamma=H \eta+\alpha$ e, se $\xi=\bigcup \xi$, então $H \eta \leq \gamma$ para todo $\eta<\xi$; tome então $\gamma=\Sigma_{\eta<\xi} H \eta+\alpha$. Defina então, conforme o caso:

$$
\begin{array}{ll}
D_{\alpha}^{\xi+1}=D_{H \xi+\alpha} & \text { se } \alpha<H(\xi+1) \\
D_{\alpha}^{\xi}=D_{\Sigma_{\eta<\xi} H \eta+\alpha} & \text { se } \alpha<H \xi \text { e } \xi=\bigcup \xi
\end{array}
$$

Então para todo $\gamma<\mu$ existem $\alpha, \xi \operatorname{com} \alpha<H(\xi)$, tais que $D_{\gamma}=D_{\alpha}^{\xi}$. Seja $p \in C$; definiremos agora uma $\lambda$-sequencia $\left\langle q_{\xi}: \xi<\lambda\right\rangle$ de condições e uma $\lambda$-sequencia $\left\langle\Pi_{\xi}: \xi<\right.$ $\lambda\rangle$ de subconjuntos de $C$ tal que

(i) $\Pi_{\xi} \subseteq C_{g \xi}$ e $\operatorname{Card}\left(\Pi_{\xi}\right) \leq H(\xi)$;

(ii) $\xi \leq \xi^{\prime} \Rightarrow q_{\xi^{\prime}} \leq q_{\xi}$,

(iii) $q_{\xi} \leq p,\left(q_{\eta}\right)_{g \xi}=\left(q_{\xi}\right)_{g \xi}$ para $\xi \leq \eta \mathrm{e}$

(iv) $\forall q^{\prime} \leq q_{\xi} \forall \alpha<H \xi \exists r \in \Pi_{\xi}\left(r\right.$ é compatível com $q^{\prime}$ e $\left.r \wedge q_{\xi} \in D_{\alpha}^{\xi}\right)$.

Suponha que $\xi=\eta+1$. Então, pelo lema 2.6.5, como $D_{\alpha}^{\xi}$ é uma seção densa de $C$ e $q_{\eta} \in C$, podemos tomar $q_{\xi}, \Pi_{\xi}$ tal que $q_{\xi} \leq q,\left(q_{\xi}\right)_{g \xi}=\left(q_{\eta}\right)_{g \xi}, \Pi_{\xi} \subseteq C_{g \xi}, \operatorname{Card}\left(\Pi_{\xi}\right)$ e $\forall q^{\prime} \leq q_{\xi} \exists r \in \Pi_{\xi}(r$ é compatível com $q^{\prime}$ e $r \wedge q \in D_{\alpha}^{\xi}$ ), isto é, teremos garantido $(i)$, (ii) e $(i v)$. Para ver que (iii) também será garantido, veja que dado $\delta<\xi$, como $g$ é crescente, pelo lema 2.6.4 temos $\left(q_{\xi}\right)_{g \delta}=\left(\left(q_{\xi}\right)_{g \xi}\right)_{g \delta}$ e, pela relação que já temos, $\left(\left(q_{\xi}\right)_{g \xi}\right)_{g \delta}=\left(\left(q_{\eta}\right)_{g \xi}\right)_{g \delta}$ e, novamente pelo lema 2.6.4, $\left(\left(q_{\eta}\right)_{g \xi}\right)_{g \delta}=\left(q_{\eta}\right)_{g \delta}$, que por hipótese de indução é igual a $\left(q_{\delta}\right)_{g \delta}$.

Suponha agora que $\xi=\bigcup \xi$. Como $C$ satisfaz a condição da $\lambda, g$-escadaria, existe $q \in C$ tal que $q \leq q_{\eta}$ e $(q)_{g \eta}=\left(q_{\eta}\right)_{g \eta}$ para todo $\eta<\xi$. Como $C_{g \xi}$ satisfaz a condição da $H(\xi)^{+}$-cadeia e $C^{g \xi}$ é $H(\xi)^{+}$-fechado, pelo argumento do lema 2.6.5 podemos tomar $q_{\xi}$ e $\Pi_{\xi}$ que garantirão $(i)$ e $(i v)$ e tais que se $\eta<\eta^{\prime} \leq \xi$ então $q_{\eta^{\prime}} \leq q_{\eta}$ (que vai garantir o item $(i i))$ e $\left(q_{\xi}\right)_{g \xi}=(q)_{g \xi}$. Para ver que também teremos $(i i)$, seja $\delta<\xi$; então $\left(q_{\xi}\right)_{g \delta}=$ $\left(\left(q_{\xi}\right)_{g \xi}\right)_{g \delta}=\left((q)_{g \xi}\right)_{g \delta}=(q)_{g \delta}=\left(q_{\delta}\right)_{g \delta}$. 
Assim, a sequencia $q_{\xi}$ está definida; como $C$ satisfaz a condição da $\lambda, g$-escadaria, existe $q \in C \operatorname{com} q \leq q_{\xi}$ e $\xi<\lambda \rightarrow\left((q)_{g \xi}=\left(q_{\xi}\right)_{g} \xi\right)$; tome $\Pi=\bigcup_{\xi<\lambda} \Pi_{\xi}$. Então $\operatorname{Card}(\Pi) \leq \mu$. Seja $\gamma<\mu$ e $q^{\prime} \leq q$; existe $\alpha<H \xi$, $\xi<\lambda$, tal que $D_{\gamma}=D_{\alpha}^{\xi}$; além disso, $q^{\prime} \leq q$ (já que $q \leq q_{\xi}$ ). Então, existe $r \in \Pi_{\xi} \subseteq \Pi$ tal que $r$ é compatível com $q^{\prime}$ e $r \wedge q_{\xi} \in D_{\gamma}$. Mas $r \wedge q \leq r \wedge q_{\xi}$, e como $D_{\gamma}$ é seção, $r \wedge q \in D_{\gamma}$.

A partir daqui, seja $M$ um $\in$-modelo transitivo tal que $C \in R^{H}$.

Lema 2.6.8 Sejam $H$ uma sequencia crescente de cardinais cofinal em $O N^{M}$, g uma sequencia de ordinais em $M$ e $C$ uma noção de forcing em $M$ satisfazendo a condição da $H \xi, g \xi$-densidade para todo $\xi \in O N^{M}$. Então todo $C$-genérico sobre $M$ é fortemente genérico sobre $M$.

Prova. Sejam $\gamma \in O N^{M}$, $\xi$ tal que $\gamma \leq H(\xi)$ e $\left\langle D_{\alpha}: \alpha<\gamma\right\rangle$ uma sequencia de seções $C$-densas em $M$. Então

$$
D=\left\{q: \forall \alpha<\gamma \forall q^{\prime} \leq q \exists r \in C_{g \xi}\left(r \text { é compatível com } q^{\prime} \text { e } r \wedge q \in D_{\alpha}\right)\right\}
$$

é $C$-denso, já que $C$ satisfaz a condição da $H \xi, g \xi$-densidade em $M$. Seja agora $q \in D \cap G$ e considere

$$
D_{\alpha}^{\prime}=\left\{p \in C_{g \xi}: p \wedge q \in D_{\alpha} \text { ou } p \text { é incompatível com } q\right\}
$$

Observe agora que se $D_{\alpha}^{\prime}$ for $C_{g \xi}$-denso, como $G_{g \xi}$ é $C_{g \xi}$-genérico, teremos $D_{\alpha}^{\prime} \cap G_{g \xi} \neq \emptyset$. Logo, tomando $p \in D_{\alpha}^{\prime} \cap G_{g \xi}$, então $p$ é compatível com $q$, já que $G_{g \xi} \subseteq G$ e $q \in G$. Daí $\exists p \in G_{g \xi}\left(p \wedge q \in D_{\alpha}\right)$, como queríamos.

Vamos mostrar então que $D_{\alpha}^{\prime}$ é $C_{g \xi}$-denso: se $p \in C_{g \xi}$ e $q$ é compatível com $p$, então existe $p \wedge q$ e $p \wedge q \leq q$, donde $\exists r \in C_{g \xi}$ tal que $r$ é compatível com $p \wedge q$ e $r \wedge q \in D_{\alpha}$. Tome agora a condição $r^{\prime}=r \wedge(q)_{\xi}$; então $r^{\prime}$ é compatível com $p$ em $C_{g \xi}$ (já que existe $r \wedge p$ ). Tome então $t \leq r^{\prime}$, p; então $t \wedge q \leq r^{\prime} \wedge q \leq r \wedge q$ o que implica que $t \wedge q \in D_{\alpha}$ e $t \in D_{\alpha}^{\prime}$.

Lema 2.6.9 Se $C$ satisfaz a condição $d a \kappa, \xi$-densidade em $M$ e $\kappa=c f^{M[G]}(\mu)$, então $\kappa=c f^{M}(\mu)$.

Prova. Pelo lema 2.3.10, $\kappa \leq c f^{M}(\mu)$. Suponha por absurdo que que $\kappa<c f^{M}(\mu)$, e seja $f^{G}: \kappa \rightarrow \mu$, cofinal. Para cada $\alpha<\kappa$ seja

$$
D_{\alpha}=\{p: \exists x(p \Vdash \exists y(y \in \hat{\kappa} \wedge\langle\hat{\alpha}, y\rangle \in f) \rightarrow(x \in \hat{\kappa} \wedge\langle\hat{\alpha}, x\rangle \in f))\} .
$$

Então $\left\langle D_{\alpha}: \alpha<\kappa\right\rangle$ é uma sequencia de seções densas em $M$. Como $C$ satisfaz a condição da $\kappa, \xi$-densidade em $M, \exists q \in G \exists \Pi \in M\left(\operatorname{Card}^{M}(\Pi) \leq \kappa\right.$ e $\forall q^{\prime} \leq q \forall \alpha<\kappa \exists p \in \Pi(p$ é compatível com $q^{\prime}$ e $\left.p \wedge q \in D_{\alpha}\right)$ ). Defina em $M$ a função $g: \Pi \times \kappa \rightarrow \mu$ por

$$
\begin{aligned}
g(r, \alpha)= & \bigcap\{\delta: r \wedge q \Vdash \exists y(y \in \hat{\kappa} \wedge\langle\hat{\alpha}, y\rangle \in f) \rightarrow(\hat{\delta} \in \hat{\kappa} \wedge\langle\hat{\alpha}, \hat{\delta}\rangle \in f)\}, \\
& \text { se } \exists \delta<\kappa(r \wedge q \Vdash \exists y(y \in \hat{\kappa} \wedge\langle\hat{\alpha}, y\rangle \in f) \rightarrow(\hat{\delta} \in \hat{\kappa} \wedge\langle\hat{\alpha}, \hat{\delta}\rangle \in f)) \\
= & 0, \text { caso contrário. }
\end{aligned}
$$

Agora, temos $\operatorname{Card}^{M}(\Pi \times \kappa)=\kappa$; seja $h \in M, h: \kappa \rightarrow \Pi \times \kappa$ bijetora. então $g \circ h: \kappa \rightarrow \mu \in M$. Seja

$$
\delta_{0}=\bigcup(g \circ h)_{*}(\kappa) .
$$


Como $\operatorname{dom}(g \circ h)=\kappa$ e $\kappa<c f^{M}(\mu), g \circ h$ não é cofinal em $\mu$, e assim $\delta_{0}<\mu$.

Seja agora $\eta=f^{G}(\gamma), \gamma<\kappa$. Então existe $s \in G$ tal que $s \Vdash \hat{\eta} \in \hat{\kappa} \wedge\langle\hat{\gamma}, \hat{\eta}\rangle \in f$ e, como $q \in G$, podemos tomar $s \leq q$ e ainda $s \Vdash F u n(f)$. Como $s \leq q$, existe $r \in \Pi$ tal que $r$ é compatível com $s$ e $r \wedge q \in D_{\gamma}$, isto é, $\exists x \in M(r \wedge q \Vdash \exists y(y \in \hat{\kappa} \wedge\langle\hat{\gamma}, y\rangle \in f) \rightarrow(x \in$ $\hat{\kappa} \wedge\langle\hat{\kappa}, x\rangle \in f)$. Por definição de $\delta_{0}$, podemos tomar $x^{G} \leq \delta_{0}$. Seja agora $s^{\prime} \in G$ tal que $s^{\prime} \leq s$ e $s^{\prime} \leq r \wedge q$. Então

(1) $\left.s^{\prime} \Vdash \exists y(y \in \hat{\kappa} \wedge \hat{\gamma}, y\rangle \in f\right) \rightarrow(x \in \hat{\kappa} \wedge\langle\hat{\gamma}, x\rangle \in f)$,

(2) $s^{\prime} \Vdash \hat{\gamma} \in \hat{\kappa} \wedge\langle\hat{\gamma}, \hat{\eta}\rangle \in f \mathrm{e}$

(3) $s^{\prime} \Vdash$ Fun $(f)$.

De (2) temos $s^{\prime} \Vdash \exists y(y \in \hat{\kappa} \wedge\langle\hat{\gamma}, y\rangle \in f)$; combinando com (1), temos

(4) $s^{\prime} \Vdash x \in \hat{\kappa} \wedge\langle\hat{\gamma}, x\rangle \in f$.

De (2), (3) e (4) segue que $x^{G}=\eta$. Mas então $\eta \leq \delta_{0}$ e, como $\eta$ foi tomado arbitrário na imagem de $f^{G}$, temos que $f^{G}$ não é cofinal em $\mu$.

Lema 2.6.10 Se $C$ satisfaz a condição da $\kappa, \xi$-densidade em $M$, então $\operatorname{Card}^{M[G]}(P(\kappa)) \leq$ $\operatorname{Card}^{M}\left(\left(C_{\xi} \times \kappa\right)^{\kappa}\right)$.

Prova. Dado $\sigma^{G} \in P^{M[G]}(\kappa)$, para cada $\alpha<\kappa$ seja

$$
D_{\alpha, \sigma}=\{p: p \Vdash \hat{\alpha} \in \sigma \vee p \Vdash \neg(\hat{\alpha} \in \sigma)\} .
$$

Então $\left\langle D_{\alpha, \sigma}: \alpha<\kappa\right\rangle$ é uma sequencia de seções densas em $M$. Vamos definir em $M[G]$ uma função $H$ tal que sua restrição a um subconjunto de $\left\{x \in P^{M}\left(C_{\xi} \times \kappa\right): \operatorname{Card}^{M}(x) \leq \kappa\right\}$ será sobrejetora em $P^{M[G]}(\kappa)$. Como

$$
\operatorname{Card}\left(\left(C_{\xi} \times \kappa\right)^{\kappa}\right)=\operatorname{Card}\left(\left\{x \in P\left(C_{\xi} \times \kappa\right): \operatorname{Card}(x) \leq \kappa\right\}\right),
$$

a tese vai seguir. Defina

$$
\begin{aligned}
H= & \left\{\langle s, \sigma\rangle: \exists p \in G \exists \Pi \subseteq C_{\xi}\left(\Pi \in M \wedge \operatorname{Card}^{M}(\Pi) \leq \kappa \wedge p \Vdash \sigma \subseteq \hat{\kappa}\right.\right. \\
& \wedge \forall \alpha<\hat{\kappa} \forall p^{\prime} \leq p \exists q \in \Pi\left(q \text { é compatível com } p^{\prime} \text { e } q \wedge p \in D_{\alpha, \sigma}\right) \\
& \wedge s=\{\langle r, \beta\rangle: r \in \Pi \wedge \beta \in \kappa \wedge(r \wedge p \Vdash \hat{\beta} \in \sigma)\})\} .
\end{aligned}
$$

Vamos provar que

$$
\sigma^{G} \in P^{M[G]}(\kappa) \rightarrow \exists s \subseteq C_{\xi} \times \kappa\left(s \in M \wedge \operatorname{Card}^{M}(s) \leq \kappa \wedge\langle s, \sigma\rangle \in H\right) .
$$

Suponha que $\sigma^{G} \subseteq \kappa$. Então $\exists p^{\prime} \in G\left(p^{\prime} \Vdash \sigma \subseteq \hat{\kappa}\right)$. Como $C$ é $\kappa, \xi$-denso em $M, \exists q \in G \exists \Pi \in$ $M, \operatorname{Card}^{M}(\Pi) \leq \kappa$ tal que

$$
\forall \alpha<\kappa \forall q^{\prime} \leq q \exists r \in \Pi\left(r \text { é compatível com } q^{\prime} \text { e } r \wedge q \in D_{\alpha, \sigma}\right) .
$$

Tome $p \leq p^{\prime}, q$. Defina então $s$ como $s=\{\langle r, \beta\rangle \in \Pi \times \kappa: r \wedge p \Vdash \hat{\beta} \in \sigma\}$.

Vamos mostrar agora que $H$ é função, isto é, que

$$
\left\langle s, \bar{\sigma}_{1}\right\rangle \in H \wedge\left\langle s, \bar{\sigma}_{2}\right\rangle \in H \rightarrow \bar{\sigma}_{1}=\bar{\sigma}_{2} .
$$

Suponha então que $\left\langle s, \bar{\sigma}_{1}\right\rangle \in H \wedge\left\langle s, \bar{\sigma}_{2}\right\rangle \in H$ e sejam $p_{1}, p_{2}, \Pi_{1}$ e $\Pi_{2}$ correspondentes de $\bar{\sigma}_{1}$ e $\bar{\sigma}_{2}$, dados na definição de $H$. Vamos mostrar que $\bar{\sigma}_{1} \subseteq \bar{\sigma}_{2}$. Seja $\gamma \in \bar{\sigma}_{1}$ e tome $p \in G$ tal que

$$
\begin{gathered}
p \Vdash \hat{\gamma} \in \bar{\sigma}_{1} \\
p \Vdash \hat{\gamma} \in \bar{\sigma}_{2} \text { ou } p \Vdash \hat{\gamma} \notin \bar{\sigma}_{2} \\
p \leq p_{1}, p_{2}
\end{gathered}
$$

Como $r \wedge p \leq r \wedge p_{1}, p$, temos que $r \wedge p_{1}$ é compatível com $p$ e assim $r \wedge p_{1} \Vdash \hat{\gamma} \in \sigma_{1}$, e portanto $\langle r, \gamma\rangle \in s$. Como $\left\langle s, \bar{\sigma}_{2} \in H, r \wedge p_{2} \Vdash \hat{\gamma} \in \sigma_{2}\right.$; mas $r \wedge p_{2}$ é compatível com $p$ e portanto $p \Vdash \hat{\gamma} \in \sigma_{2}$. Como $p \in G, \gamma \in \bar{\sigma}_{2}$. Por simetria, $\bar{\sigma}_{2} \subseteq \bar{\sigma}_{1}$. 


\subsection{A independência da HGC e o teorema de Easton}

Um modelo para ZF2 +HGC. Nesta seção, vamos definir duas classes: na primeira, conseguiremos um genérico que terá todas as bijeções que irão satisfazer a HGC e, com a segunda, provaremos o teorema de Easton.

Definição 2.7.1 $\beth$ é a sequencia definida por:

$$
\begin{aligned}
& \beth_{0}=\omega \\
& \beth_{\alpha+1}=\operatorname{Card}\left(P\left(\beth_{\alpha}\right)\right) \\
& \beth_{\lambda}=\bigcup\left\{\beth_{\alpha}: \alpha<\lambda\right\} \text { se } \lambda \text { é limite. }
\end{aligned}
$$

Como só usaremos $\beth$ em $M$, assumiremos que ela está definida em $M$, e assim evitaremos ter que escrever $\beth^{M}$ a todo momento.

Definição 2.7.2 Sejam, em $M$ :

(a) $Q_{\alpha}=\left\{\langle\xi, \alpha, \eta\rangle: \xi<\beth_{\alpha}^{+} \wedge \eta<\beth_{\alpha+1}\right\}$

(b) $C$ é a classe dos $p$ tais que

(i) $p \subseteq \bigcup\left\{Q_{\beta}: \beta<\alpha\right\}$ para algum $\alpha$.

(ii) $\langle\xi, \alpha, \eta\rangle \in p \wedge\left\langle\xi, \alpha, \eta^{\prime}\right\rangle \in p \rightarrow \eta=\eta^{\prime}$.

(iii) $\operatorname{Card}\left(p \cap Q_{\alpha}\right) \leq \beth_{\alpha}$ para todo $\alpha$.

(c) Para todos $p, q \in C, p \leq q$ se, e somente se, $q \subseteq p$.

(d) se $p \in C$,

$$
\begin{gathered}
(p)_{\alpha}=p \cap \bigcup\left\{Q_{\beta}: \beta<\alpha\right\},(p)^{\alpha}=p-(p)_{\alpha}, \\
C_{\alpha}=\left\{(p)_{\alpha}: p \in C\right\}, C^{\alpha}=\left\{(p)^{\alpha}: p \in C\right\}
\end{gathered}
$$

Lema 2.7.3 O conjunto $C$ da definição 2.7.2 é uma noção coerente de forcing em $M$.

Prova. (i) para todo ordinal $\alpha$, temos $C_{\alpha} \subseteq C$, donde $\bigcup C_{\alpha} \subseteq C$; para ver a inclusão contrária, seja $p \in C$; então $\exists \alpha\left(p \subseteq \bigcup_{\beta<\alpha} Q_{\beta}\right)$. Logo $(p)_{\alpha}=p \cap \bigcup_{\beta<\alpha} Q_{\beta}=p$, isto é, $p \in C_{\alpha}$. Se $\alpha<\gamma$, dado $p \in C$, vamos mostrar que $(p)_{\alpha} \in C_{\gamma}$. Tome $q=p-\left(p \cap \bigcup_{\alpha \leq \beta<\gamma} Q_{\beta}\right)$; então $(q)_{\gamma}=p \cap \bigcup_{\beta<\alpha}=(p)_{\alpha}$, e como $q \subseteq p$, temos $q \in C$ pela definição 2.4.2(b).

(ii) Tome $(p)_{\alpha}$ e $(q)^{\alpha}$; então $(p)_{\alpha} \wedge(q)^{\alpha}=(p)_{\alpha} \cup(q)^{\alpha}=\left(p \cap \bigcup_{\beta<\alpha} Q_{\beta}\right) \cup\left(q-(q)_{\alpha}\right)=(p \cap$ $\left.\bigcup_{\beta<\alpha} Q_{\beta}\right) \cup\left(q-\left(q \cap \bigcup_{\beta<\alpha} Q_{\beta}\right)\right) \in C$, como é fácil de notar. Para ver que $f_{\alpha}$ é isomorfismo, note primeiro que $f_{\alpha}\left\langle(p)_{\alpha},(p)^{\alpha}\right\rangle=p$, e portanto $f_{\alpha}$ é sobrejetora e, se $\left\langle(p)_{\alpha},(q)^{\alpha}\right\rangle \neq\left\langle(r)_{\alpha},(s)^{\alpha}\right\rangle$, então $(p)_{\alpha} \cup(q)^{\alpha} \neq(r)_{\alpha} \cup(s)^{\alpha}$ : de fato, se existe $x$ tal que $x \in(p)_{\alpha}-(r)_{\alpha}$, isto é, se $x \in p \cap \bigcup_{\beta<\alpha} Q_{\beta}$ mas $x \notin r \cap \bigcup_{\beta<\alpha} Q_{\beta}$ e valesse a igualdade, teríamos $x \in(s)^{\alpha}$, isto é, $x \in s$ e $x \notin \bigcup_{\alpha<\beta} Q_{\beta}$, um absurdo $\operatorname{com} x \in(p)_{\alpha}$. Se $x \in(q)^{\alpha}-(s)^{\alpha}$ e vale a igualdade, então $x \in(r)_{\alpha}$ e temos um absurdo semelhante ao outro. Se $p \leq q$ e $r \leq s$, então $f_{\alpha}\langle p, q\rangle=p \wedge q \leq$ $r \wedge s=f_{\alpha}\langle r, s\rangle$.

Lema 2.7.4 Se $\alpha$ é sucessor, $C_{\alpha}$ satisfaz a condição da $\beth_{\alpha}^{+}$-cadeia.

Prova. Vamos mostrar que, se $\alpha=\delta+1$, então $\operatorname{Card}\left(C_{\alpha}\right)=\beth_{\alpha}$; o resultado segue trivialmente. Veja que

$$
p \leftrightarrow\left\{\begin{array}{l}
p \subseteq \bigcup_{\beta<\alpha} Q_{\beta} \\
p \text { é função de duas variáveis e } \\
\forall \gamma\left(\operatorname{Card}\left(p \cap Q_{\gamma}\right) \leq \beth_{\gamma}\right) .
\end{array}\right.
$$

e, como $\alpha=\delta+1$, isto equivale a 


$$
\exists a\left(a \subseteq \beth_{\delta}^{+} \times(\delta+1) \wedge F u n(p) \wedge \operatorname{dom}(p)=a \wedge \operatorname{Im}(p) \subseteq \beth_{\delta+1} \wedge \forall \gamma\left(\operatorname{Card}\left(p \cap Q_{\gamma}\right) \leq \beth_{\gamma}\right) .\right.
$$

Se $I=\{\gamma \leq \delta: \exists \beta(\langle\beta, \gamma\rangle \in \operatorname{dom}(p))\}$, podemos definir, para cada $\gamma \in I, p_{\gamma}(-)=p(-, \gamma)$, e temos $\operatorname{dom}(p)=\bigcup_{\gamma \in I} \operatorname{dom}\left(p_{\gamma}\right)$. Agora $\operatorname{Card}\left(p \cap Q_{\gamma}\right)=\operatorname{Card}\left(p_{\gamma}\right)=\operatorname{Card}\left(\operatorname{dom}\left(p_{\gamma}\right)\right)$, para cada $\gamma \in I$; logo $\operatorname{Card}(\operatorname{dom}(p))=\operatorname{Card}\left(\bigcup_{\gamma \in I} \operatorname{dom}\left(p_{\gamma}\right)\right)=\Sigma_{\gamma \in I} \operatorname{Card}\left(\operatorname{dom}\left(p_{\gamma}\right)\right) \leq \Sigma_{\gamma \in I} \beth_{\gamma}=$ $\sup _{\gamma \in I} \beth_{\gamma} \leq \beth_{\delta}$. Como $\operatorname{Card}\left(\beth_{\delta}^{+} \times(\delta+1)\right)=\beth_{\delta}^{+}$, temos $\operatorname{Card}\left(P_{\leq} \beth_{\delta}\left(\beth_{\delta}^{+}\right)\right)$possibilidades pra $\operatorname{dom}(p)$, no máximo. Agora $\operatorname{Card}\left(P_{\leq \beth_{\delta}}\left(\beth_{\delta}^{+}\right)\right)=\sup \left\{\left(\beth_{\delta}^{+}\right)^{\mu}: \operatorname{Card}(\mu) \wedge \mu \leq \beth_{\delta}\right\}=\left(\beth_{\delta}^{+}\right)^{\beth_{\delta}}$; assim, $\left(\beth_{\delta}^{+}\right)^{\beth_{\delta}}$ é um limitante superior para a quantidade de possíveis domínios para os $p \in C_{\alpha}$. Agora, para cada domínio a possível, temos $\operatorname{Card}\left(\beth_{\delta+1}^{a}\right)$ funções $p \in C_{\alpha}$; logo, temos no máximo

$$
\left(\beth_{\delta}^{+}\right)^{\beth_{\delta}} \cdot \beth_{\delta+1}^{\beth_{\delta}}=\beth_{\delta+1}^{\beth_{\delta}}=\left(2^{\beth_{\delta}}\right) \beth_{\delta}=2^{\beth_{\delta} \cdot \beth_{\delta}}=2^{\beth_{\delta}}=\beth_{\delta+1}=\beth_{\alpha}
$$

elementos em $C_{\alpha}$. Vamos mostrar agora que $\beth_{\alpha}$ também é um limitante inferior para a cardinalidade de $C_{\alpha}$. Tomando apenas os $p \in C_{\alpha}$ tais que $p=p_{\delta}$ e $\operatorname{Card}\left(p_{\delta}\right)=\beth_{\delta}$, temos $\operatorname{Card}(p)=\beth_{\delta}$. Assim temos $\operatorname{Card}\left(P_{=\beth_{\delta}}\left(\beth_{\delta}^{+}\right)\right)=\left(\beth_{\delta}^{+}\right)^{\beth_{\delta}}$ possibildades para os domínios destes $p$ e, para cada um destes domínios, temos $\operatorname{Card}\left(\beth_{\delta+1}^{\text {dom }(p)}\right)=\beth_{\delta+1}^{\beth_{\delta}}$ destas $p$ e, assim, temos ao menos $\left(\beth_{\delta}^{+}\right)^{\beth_{\delta}} \cdot \beth_{\delta+1}^{\beth_{\delta}}=\beth_{\alpha}$ elementos.

Lema 2.7.5 Vale que

$$
\begin{array}{ll}
\operatorname{Card}\left(C_{\alpha}\right)=\beth_{\alpha}, & \text { se } \alpha \text { não é limite, } \\
\operatorname{Card}\left(C_{\alpha}\right) \leq \beth_{\alpha+1}, & \text { se } \alpha \text { é limite }
\end{array}
$$

e assim, se $\alpha$ não é limite, $C_{\alpha}$ satisfaz a condição da $\beth_{\alpha}^{+}-$cadeia. Além disso, também temos que $C^{\alpha}$ é $\beth_{\alpha}^{+}-$fechado.

Prova. Dado $E \subseteq C^{\alpha}$ com $\operatorname{Card}(E)<\beth_{\alpha}^{+}$, linearmente ordenado, podemos tomar uma sequencia decrescente $\left\langle p_{\xi}: \xi<\kappa\right\rangle, \kappa \leq \beth_{\alpha}^{+}$, tal que $\exists q \in E \forall p \in E\left(p \leq q \wedge \exists \xi\left(p_{\xi}=p\right)\right)$; de fato, tome $p=\bigcup p_{\xi}$. Então $p$ é uma condição: $(i)$ tome $\alpha=\sup \left\{\sigma: \exists \xi\left(p_{\xi} \subseteq \bigcup_{\beta<\sigma}\right)\right\}$. Se $\langle\xi, \gamma, \eta\rangle \in p$, então $\exists \delta\left(\langle\xi, \gamma, \eta\rangle \in p_{\delta}\right)$ e assim $\langle\xi, \gamma, \eta\rangle \in \bigcup_{\beta<\sigma} Q_{\beta}$, donde $p \subseteq \bigcup_{\beta<\sigma} Q_{\beta}$. (ii) É claro que $\langle\xi, \alpha, \eta\rangle \in p \wedge\left\langle\xi, \alpha, \eta^{\prime}\right\rangle \in p \rightarrow \eta=\eta^{\prime}$. (iii) Dado um ordinal $\delta, \operatorname{Card}\left(p \cap Q_{\delta}\right)=$ $\operatorname{Card}\left(\bigcup_{\xi} p_{\xi} \cap Q_{\delta}\right)=\operatorname{Card}\left(\bigcup_{\xi}\left(p_{\xi} \cap Q_{\delta}\right)\right)$. Agora, se $\kappa \leq \beth_{\delta}$, então como $\forall \xi\left(\operatorname{Card}\left(p_{\xi} \cap Q_{\delta}\right) \leq\right.$ $\left.\beth_{\delta}\right)$, temos $\operatorname{Card}\left(\bigcup_{\xi}\left(p_{\xi} \cap Q_{\delta}\right)\right) \leq \beth_{\delta}$. Agora, se $\beth_{\delta}<\kappa$, então como $p_{\xi}$ é decrescente, temos que $\bigcup p_{\xi}=\bigcup\left\{p_{\xi}: \beth_{\delta} \leq \gamma \leq \xi<\kappa\right\}$, para algum $\gamma$ tal que $\operatorname{Card}\left(\left\{p_{\xi}: \gamma \leq \xi<\kappa\right\}\right)<\beth_{\delta}$, donde $\operatorname{Card}\left(\bigcup_{\xi}\left(p_{\xi} \cap Q_{\delta}\right)\right) \leq \beth_{\delta}$.

Lema 2.7.6 Se $\alpha$ for limite e Id for a sequencia identidade em $\alpha$, então $C$ satisfaz a condição da $\alpha, I d_{\alpha}$-escadaria.

Prova. Tome $\gamma<\alpha$ e $\left\langle p_{\xi}: \xi<\gamma\right\rangle$ uma sequencia decrescente tal que se $\xi \leq \eta<\gamma$ então $\left(p_{\eta}\right)_{\xi}=p_{\xi}$. Defina $r=\bigcup_{\eta<\gamma} p_{\eta}$. Então $(r)_{\xi}=r \cap \bigcup_{\beta<\xi} Q_{\beta}=\bigcup_{\eta<\gamma} p_{\eta} \cap \bigcup_{\beta<\xi} Q_{\beta}=$ $\bigcup_{\eta<\gamma}\left(p_{\eta} \cap \bigcup_{\beta<\xi} Q_{\beta}\right)=\bigcup_{\eta<\gamma}\left(p_{\eta}\right)_{\xi}=\bigcup_{\eta<\xi}\left(p_{\eta}\right)_{\xi} \cup \bigcup_{\xi \leq \eta<\gamma}\left(p_{\eta}\right)_{\xi}=\bigcup_{\eta<\xi}\left(p_{\eta}\right)_{\xi} \cup p_{\xi}$. Agora $\eta<\xi \rightarrow p_{\eta} \leq p_{\xi}$ e então $\left(p_{\eta}\right)_{\xi} \leq\left(p_{\xi}\right)_{\xi}=p_{\xi}$, donde $(r)_{\xi}=(p)_{\xi}$.

Corolário 2.7.7 C satisfaz a condição da $\beth_{\alpha}, \alpha$-densidade para todo $\alpha$. Consequentemente, todo $C$-genérico sobre $M$ é fortemente genérico.

Prova. Se $\alpha$ não é limite, então pelo lema 2.7.4, $C_{\alpha}$ satisfaz a condição da $\beth_{\alpha}^{+}$-cadeia e, se $\alpha$ é limite, $C_{\alpha}$ satisfaz a condição da $\beth_{\alpha}^{+}$-cadeia pelo lema 2.7.5. Por este mesmo lema, $C^{\alpha}$ sempre é $\beth_{\alpha}^{+}$-fechado. Logo, o resultado segue do lema 2.6.6. 
Corolário 2.7.8 Se Gé um C-genérico sobre $M$, então $M[G] \vDash 2^{\aleph_{\alpha}}=\aleph_{\alpha+1}$ para todo $\alpha$.

Prova. Seja $G$ um $C$-genérico sobre $M$; pelo lema 2.6.8, $G$ é fortemente genérico. Assim, $M[G] \vDash Z F 2$ e, pelo lema 2.6.10,

$$
(*) \operatorname{Card}\left(P\left(\beth_{\alpha}\right)\right)^{M[G]} \leq \operatorname{Card}^{M}\left(\left(C_{\alpha} \times \beth_{\alpha}\right)^{\beth_{\alpha}}\right) \leq \beth_{\alpha+1} .
$$

Agora, considere $F_{\alpha}=\left\{\langle\xi, \eta\rangle \in \beth_{\alpha}^{+} \times \beth_{\alpha+1}:\langle\xi, \alpha, \eta\rangle \in \bigcup G\right\}$. Então $F_{\alpha} \in M[G]$ é uma bijeção já que

$$
D_{\eta}=\left\{p:\langle\xi, \alpha, \eta\rangle \in p \wedge \xi \in \beth_{\alpha}^{+}\right\} \text {é denso para todo } \eta \in \beth_{\alpha+1} \text {. }
$$

Vamos provar agora por indução em $\alpha$ que $\operatorname{Card}^{M[G]}\left(\beth_{\alpha}\right)=\aleph_{\alpha}^{M[G]}$. Para $\alpha=0$ é claro; temos ainda, em $M[G]$, usando as $F_{\alpha}$, que $\operatorname{Card}\left(\beth_{\alpha+1}\right)=\operatorname{Card}\left(\beth_{\alpha}^{+}\right)$. Agora, pelo lema 2.6.9, temos

$$
c f^{M[G]}\left(\beth_{\alpha}^{+}\right)>\beth_{\alpha},
$$

e $\operatorname{assim} \operatorname{Card}\left(\beth_{\alpha}^{+}\right)>\beth_{\alpha}=\aleph_{\alpha}$, donde $\operatorname{Card}\left(\beth_{\alpha+1}\right)>\aleph_{\alpha}$. Logo $\operatorname{Card}\left(\beth_{\alpha+1}\right)=\aleph_{\alpha+1}$. Se $\alpha$ for limite,

$$
\operatorname{Card}\left(\beth_{\alpha}\right)=\Sigma_{\beta<\alpha} \operatorname{Card}\left(\beth_{\beta}\right)=\Sigma_{\beta<\alpha} \operatorname{Card}\left(\aleph_{\beta}\right)=\aleph_{\alpha} .
$$

Segue de $(*)$ que $\operatorname{Card}\left(P\left(\aleph_{\alpha}\right)\right)=\operatorname{Card}\left(P\left(\beth_{\alpha}\right)\right) \leq \operatorname{Card}\left(\beth_{\alpha+1}\right)=\aleph_{\alpha+1}$.

O teorema de Easton. Com este útlimo resultado encerraremos nossa discussão sobre a Lógica de Segunda Ordem e a Hipótese do Contínuo.

Teorema 2.7.9 Seja $M$ um $\in$-modelo transitivo enumerável de $Z F 2$ tal que $M \vDash H G C$, e seja $H \in F^{H}$ tal que para todos os cardinais regulares $\kappa, \lambda$ de $M$,

(i) $H(\kappa)$ é um cardinal regular de $M$.

(ii) Se $\kappa \leq \lambda$, então $H(\kappa) \leq H(\lambda)$.

(iii) $\kappa<c f^{M}(H(\kappa))$.

Então existe uma noção de forcing $C \in R^{H}$ tal que, para todo $G \subseteq C$ fortemente genérico, temos

(a) $c f^{M}=c f^{M[G]} e$

(b) $M[G] \vDash 2^{\kappa}=H(\kappa)$, para todo cardinal regular $\kappa$ de $M[G]$.

Prova. Seja $\left\langle\kappa_{\xi}: \xi \in O N^{M}\right\rangle$ a sequencia crescente de todos os cardinais regulares de $M$ e seja, para cada $\xi \in O N^{M}$,

$$
Q_{\xi}=\left\{\left\langle\kappa_{\eta}, \alpha, \beta\right\rangle: \eta \leq \xi \wedge \alpha<H\left(\kappa_{\xi}\right) \wedge \beta<\alpha\right\} .
$$

Seja $C$ a classe de todas as funções $p \in M$ tais que

(1) $\operatorname{Im}(p) \subseteq 2$,

(2) $\exists \xi\left(\operatorname{dom}(p) \subseteq Q_{\xi}\right) \mathrm{e}$

(3) $\forall \xi\left(C \operatorname{Card}\left(\operatorname{dom}(p) \cap Q_{\xi}\right)<\kappa_{\xi}\right)$,

e seja $p \leq q \Leftrightarrow q \subseteq p$. Para cada $p \in C$ sejam

$$
\begin{array}{cl}
(p)_{\xi}=p \nmid Q_{\xi}, & (p)^{\xi}=p-(p)_{\xi}, \\
C_{\xi}=\left\{(p)_{\xi}: p \in C\right\}, & C^{\xi}=\left\{(p)^{\xi}: p \in C\right\} .
\end{array}
$$

Lema 2.7.10 $C$ é uma noção de forcing coerente. 
Prova. Vamos checar os três itens da definição 2.6.1.

(i) Seja $p \in C$; então existe $\xi$ tal que $\operatorname{dom}(p) \subseteq Q_{\xi}$, e assim $p=p \uparrow Q_{\xi}=(p)_{\xi} \in C_{\xi}$, donde $C=\bigcup C_{\xi}$, e assim $p=p \uparrow Q_{\xi}=(p)_{\xi} \in C_{\xi}$, donde $C=\bigcup_{\xi} C_{\xi}$, e é claro que $\alpha \leq \beta \rightarrow C_{\alpha} \subseteq C_{\beta}$.

(ii) Sejam $(p)_{\xi} \in C_{\xi}$ e $(q)^{\xi} \in C^{\xi}$. Então $r=(p)_{\xi} \cup(q)^{\xi}=p \nmid Q_{\xi} \cup\left(q-q \uparrow Q_{\xi}\right)$ e, assim, $r$ é uma função com domínio igual a $\operatorname{dom}(q)$ e com imagem em 2, e portanto satisfaz as condições (1), (2) e (3) dos elementos de $C$. Para ver que $f_{\xi}: C_{\xi} \times C^{\xi} \rightarrow C$ dado por $f_{\xi}(p, q)=p \cup q$ é um isomorfismo, repare que (1) $f$ é sobrejetora: $f_{\xi}\left\langle(p)_{\xi},(p)^{\xi}\right\rangle=p$. (2) $f_{\xi}$ é injetora: se $f_{\xi}\left\langle(p)_{\xi},(q)^{\xi}\right\rangle=f_{\xi}\left\langle(r)_{\xi},(s)^{\xi}\right\rangle$, então $p\left\lceil Q_{\xi} \cup\left(q-q\left\lceil Q_{\xi}\right)=r \uparrow Q_{\xi} \cup\left(s-s\left\lceil Q_{\xi}\right)\right.\right.\right.$, e $\operatorname{assim} p \nmid Q_{\xi}=r \uparrow Q_{\xi}$ e $\left(q-q \uparrow Q_{x} i\right)-\left(s-s \uparrow Q_{\xi}\right)$, donde $(p)_{\xi}=(r)_{\xi}$ e $(q)^{\xi}=(s)^{\xi}$. $(3) f_{\xi}$ preserva a ordem: se $\left\langle(p)_{\xi},(q)^{\xi}\right\rangle \leq\left\langle(r)_{\xi},(s)^{\xi}\right\rangle$, isto é o mesmo que $(r)_{\xi} \subseteq(p)_{\xi}$ e $(s)^{\xi} \subseteq(q)^{\xi}$, donde $(r)_{\xi} \cup(s)^{\xi} \subseteq(p)_{\xi} \cup(q)^{\xi}$, isto é, $f_{\xi}\left\langle(p)_{\xi},(q)^{\xi}\right\rangle \leq f_{\xi}\left\langle(r)_{\xi},(s)^{\xi}\right\rangle$.

(iii) É claro.

Lema 2.7.11 Para todo $\xi, C^{\xi}$ é $\kappa_{\xi}^{+}$-fechado.

Prova. De fato, seja $\gamma \leq \kappa_{\xi}$ e $\left\langle\left(p_{\alpha}\right)^{\xi}: \alpha<\gamma\right\rangle$ uma sequencia decrescente de condições em $C^{\xi}$. Então $q=\bigcup_{\alpha<\gamma}\left(p_{\alpha}\right)^{\xi}$ é uma função com domínio $\bigcup_{\alpha<\gamma} \operatorname{dom}\left(p_{\alpha}\right) \cap Q_{\xi}$, com imagem em 2 e, dado $\beta$,

Caso 1. $\beta \leq \xi$ : Então $\operatorname{Card}\left(\operatorname{dom}(q) \cap Q_{\beta}\right)=\operatorname{Card}\left(\bigcup_{\alpha<\gamma} \operatorname{dom}\left(p_{\alpha}\right) \cap Q_{\beta}\right)$. Agora, temos que $\operatorname{Card}\left(\bigcup_{\alpha<\gamma} \operatorname{dom}\left(p_{\alpha}\right) \cap Q_{\beta}\right) \leq \sup _{\alpha<\gamma} \operatorname{Card}\left(\operatorname{dom}\left(p_{\alpha}\right) \cap Q_{\beta}\right)$. Como $\alpha \leq \alpha^{\prime} \rightarrow \operatorname{dom}\left(p_{\alpha}\right) \subseteq$ $\operatorname{dom}\left(p_{\alpha^{\prime}}\right)$, temos

$$
\sup _{\alpha<\gamma} \operatorname{Card}\left(\operatorname{dom}\left(p_{\alpha}\right) \cap Q_{\beta}\right)=\bigcup_{\alpha<\gamma} \operatorname{Card}\left(\operatorname{dom}\left(p_{\alpha}\right) \cap Q_{\beta}\right) .
$$

Mas Id : $\bigcup_{\alpha<\gamma} \operatorname{Card}\left(\operatorname{dom}\left(p_{\alpha}\right) \cap Q_{\beta}\right) \rightarrow \operatorname{Card}\left(\bigcup_{\alpha<\gamma} \operatorname{dom}\left(p_{\alpha}\right) \cap Q_{\beta}\right)$ é injetora. Logo

$$
\operatorname{Card}\left(\bigcup_{\alpha<\gamma} \operatorname{dom}\left(p_{\alpha}\right) \cap Q_{\beta}\right)=\sup _{\alpha<\gamma} \operatorname{Card}\left(\operatorname{dom}\left(p_{\alpha}\right) \cap Q_{\beta}\right),
$$

e $\operatorname{assim} \operatorname{Card}\left(\operatorname{dom}(q) \cap Q_{\beta}\right)=\sup _{\alpha<\gamma}\left(\kappa_{\beta}\right)=\kappa_{\beta}$.

Caso 2. $\xi<\beta: \operatorname{Card}\left(\operatorname{dom}(q) \cap Q_{\beta}\right)=\operatorname{Card}\left(\bigcup_{\alpha<\gamma} \operatorname{dom}\left(p_{\alpha}\right) \cap Q_{\xi}\right)=\kappa_{\xi} \leq \kappa_{\beta}$.

Lema 2.7.12 Para todo $\xi, C_{\xi}$ satisfaz a condição da $\kappa_{\xi}^{+}$-cadeia.

Prova. Seja $I \subseteq C_{\xi}$ um conjunto de condições incompatíveis. Vamos primeiro provar que existe $A \subseteq Q_{\xi}$ tal que

$$
I \subseteq F(A, 2) \text { e } \operatorname{Card}(A)=\kappa_{\xi},
$$

onde $F(A, 2)$ é o conjunto de todas as funções com domínio contido em $A$ e de cardinalidade menor que $\kappa_{\xi}$ e com imagem em 2. Defina, por recursão, uma sequencia $\left\langle A_{\alpha}: \alpha \leq \kappa_{\xi}\right\rangle$ tal que

(i) $A_{0}=0$

(ii) $A_{\alpha}=\bigcup_{\beta<\alpha} A_{\beta}$ se $\beta$ é limite e

(iii) Para cada $p \in F\left(A_{\alpha}, 2\right)$ tome, se houver, $q_{p} \in I$ tal que $q_{p}\left\lceil A_{\alpha}=p\right.$ e seja

$$
A_{\alpha+1}=A_{\alpha} \cup \bigcup\left\{\operatorname{dom}\left(q_{p}\right): p \in F\left(A_{\alpha}, 2\right)\right\} .
$$

Vamos provar que $\operatorname{Card}\left(A_{\alpha}\right) \leq \kappa_{\xi}$ para todo $\alpha \leq \kappa_{\xi}$; tomando $A=A_{\xi}$, vai seguir o resultado desejado.

É claro que se o resultado é válido se $\alpha$ é 0 ou limite; para ver que é válido para $\alpha+1$, suponha que $\operatorname{Card}\left(A_{\alpha}\right) \leq \kappa_{\xi}$. Então 


$$
\operatorname{Card}\left(F\left(A_{\alpha+1}, 2\right)\right) \leq \kappa_{\xi}^{<\kappa_{\xi}} \cdot 2=\kappa_{\xi},
$$

já que $M \vDash H G C$. Assim,

$$
\operatorname{Card}\left(A_{\alpha+1}\right) \leq \operatorname{Card}\left(A_{\alpha}\right)+\operatorname{Card}\left(F\left(A_{\alpha}, 2\right)\right) \cdot \kappa_{\xi} \leq \kappa_{\xi} .
$$

Em particular, $\operatorname{Card}\left(F\left(A_{\kappa_{\xi}}, 2\right)\right) \leq \kappa_{\xi}$. Vamos mostrar agora que $\left.I \subseteq F\left(A_{\kappa_{\xi}}, 2\right)\right)$. Seja $p \in I$; como $\operatorname{Card}(\operatorname{dom}(p))<\kappa_{\xi}$, existe $\alpha$ tal que $\operatorname{dom}(p) \cap A_{\alpha}=\operatorname{dom}(p) \cap A_{\alpha+1}$. Seja $q \in I$ tal que $q \uparrow A_{\alpha}=p$ e $\operatorname{dom}(q) \subseteq A_{\alpha}+1$. Vamos mostrar que $p$ e $q$ são compatíveis; como $p, q \in I$, vai seguir que $p=q$. Seja $x \in \operatorname{dom}(p) \cap \operatorname{dom}(q)$; então $x \in \operatorname{dom}(p) \cap A_{\alpha+1}$. Segue que $p(x)=q(x)$; mas como $x$ foi tomado arbitrário em $\operatorname{dom}(p) \cap \operatorname{dom}(q)$, segue que $p=q$. $\operatorname{Logo} \operatorname{dom}(p) \subseteq A_{\alpha+1}$ e, assim, $p \in F\left(A_{\kappa_{\xi}}, 2\right)$.

Corolário 2.7.13 (a) $C_{\xi}$ satisfaz a condição da $\kappa_{\xi}, \xi$-densidade para todo $\xi$.

(b) $c f^{M}=c f^{M[G]}$.

(c) $M$ e $M[G]$ tem os mesmos cardinais.

Corolário 2.7.14 $M[G] \vDash \forall \xi\left(2^{\kappa_{\xi}}=H\left(\kappa_{\xi}\right)\right)$.

Prova. Seja $J=\bigcup G \in R[G]^{H}$ e defina $B_{\alpha}=\left\{\beta \in \kappa_{\xi}: J\left(\kappa_{\xi}, \alpha, \beta\right)=0\right\}$. Dados $\alpha, \alpha^{\prime}<$ $H\left(\kappa_{\xi}\right)$ com $\alpha \neq \alpha^{\prime}$, a classe

$$
D=\left\{p \in C: \exists \beta\left(p\left(\kappa_{\xi}, \alpha, \beta\right) \text { e } p\left(\kappa_{\xi}, \alpha^{\prime}, \beta\right) \text { estão definidos e são diferentes }\right\}\right.
$$

é densa, e assim $B_{\alpha} \neq B_{\alpha^{\prime}}$.

Além disso, $B_{\alpha} \subseteq \kappa_{\xi}$ em $M[G]$, para todo $\alpha<H\left(\kappa_{\xi}\right)$. Logo, para cada $\alpha<H\left(\kappa_{\xi}\right)$, $J\left(\kappa_{\xi}, \alpha,-\right)$ é uma função de $\kappa_{\xi}$ em 2 , e assim $M[G] \vDash H\left(\kappa_{\xi}\right) \leq 2^{\kappa_{\xi}}$.

Por outro lado, pelo lema 2.6.10, temos que $M[G] \vDash 2^{\kappa_{\xi}} \leq \operatorname{Card}^{M}\left(\left(C_{\xi} \times \kappa_{\xi}\right)^{\kappa_{\xi}}\right)$. Mas $\operatorname{Card}^{M}\left(C_{\xi}\right) \leq \operatorname{Card}^{M}\left(F\left(Q_{\xi}, 2\right)\right)=\left(H\left(\kappa_{\xi}\right)^{<\kappa_{\xi}}\right)^{M}=H\left(\kappa_{\xi}\right)$, já que $M \vDash H G C$ (Jech (2006)). Segue que $M \vDash C \operatorname{Card}\left(\left(C_{\xi} \times \kappa_{\xi}\right)^{\kappa_{\xi}}\right)=\left(H\left(\kappa_{\xi}\right) \cdot \kappa_{\xi}\right)^{\kappa_{\xi}}=H\left(\kappa_{\xi}\right)$. Logo $M[G] \vDash 2^{\kappa_{\xi}} \leq H\left(\kappa_{\xi}\right)$. 


\section{Capítulo 3}

\section{Outros Tópicos}

\subsection{O Teorema de Lindström}

Vamos provar que sob a concepção adotada a respeito do que é uma lógica que qualquer lógica satisfazendo as propriedades da Compacidade e de Löwenheim-Skolem (para pelo menos conjuntos enumeráveis de sentenças) e que "contenha" a Lógica de Primeira Ordem, não é uma Lógica mais "forte" do que a Lógica de Primeira Ordem. Vamos tornar estas noções precisas.

Definição 3.1.1 $\operatorname{Mod}_{L}^{V}$ é a classe de todas as $V$-estruturas da lógica L. $\operatorname{Mod}_{L}^{V}(\varphi)=$ $\left\{V\right.$-estrutras $\left.M: M \vDash_{L} \varphi\right\}$. Se $\Gamma \cup\{\varphi\} \subseteq L(V)$, definimos a relação de consequência semântica:

$$
\Gamma \vDash_{L} \varphi \Leftrightarrow \text { para todo } M \in \operatorname{Mod}_{L}^{V} \text {, se } M \vDash_{L} \Gamma \text {, então } M \vDash_{L} \varphi \text {. }
$$

Definição 3.1.2 Se $M$ e $N$ são $V$-estruturas, então $M$ e $N$ são L-equivalentes, em símbolos $M \equiv_{L} N$, se e somente se para toda $\varphi \in L(V), M \vDash_{L} \varphi \Leftrightarrow N \vDash_{L} \varphi$.

As propriedades a seguir servem para garantir que qualquer lógica que iremos considerar tem, ao menos, tanto poder expressivo quanto a Lógica de Primeira Ordem.

Definição 3.1.3 (i) Para todo $V$ e toda $\varphi \in L 1(V)$, existe $\psi \in L(V)$ tal que

$$
\operatorname{Mod}_{L}^{V}(\psi)=\operatorname{Mod}_{L 1}^{V}(\varphi)
$$

(ii) Para todo $V$ e toda $\varphi \in L(V)$, existe $\psi \in L(V)$ tal que

$$
\operatorname{Mod}_{L}^{V}(\psi)=\operatorname{Mod}_{L}^{V}-\operatorname{Mod}_{L}^{V}(\varphi) .
$$

(iii) Para todo $V$ e todas $\varphi, \chi \in L(V)$, existe $\psi \in L(V)$ tal que

$$
\operatorname{Mod}_{L}^{V}(\psi)=\operatorname{Mod}_{L}^{V}(\varphi) \cap \operatorname{Mod}_{L}^{V}(\chi)
$$

(iv) Se vé um simbolo de constante (de função n-ária, de relação n-ária) e $v \in V$, então para toda $\varphi \in L(V)$ existe $\psi \in L(V-\{c\})$ tal que para todas as $(V-\{v\})$-estruturas $M$, $M \vDash \psi \Leftrightarrow\langle M, a\rangle \vDash \varphi$ para algum $a \in M$ (a uma função n-ária de $M$ em $M$, a uma relação $n$-ária em $M)$.

Frequentemente iremos indicar fórmulas correspondentes pelo mesmo símbolo. Por exemplo, se $\varphi \in L 1(V), \psi \in L(V)$ e $\operatorname{Mod}_{L}^{V}(\psi)=\operatorname{Mod}_{L 1}^{V}(\varphi)$, escreveremos $\varphi$ no lugar de $\psi$. 
Definição 3.1.4 Uma fórmula $\varphi \in L^{\prime}(V)$ é equivalente a $\psi \in L(V)$, em símbolos $\varphi \equiv_{V} \psi$, se $\operatorname{Mod}_{L^{\prime}}^{V}(\varphi)=\operatorname{Mod}_{L}^{V}(\psi)$ (assim, fórmulas equivalentes tem "o mesmo sentido"). A lógica $L^{\prime}$ é mais forte do que a lógica $L$, em símbolos $L \leq L^{\prime}$, se toda $\psi \in L(V)$ é equivalente a uma $\varphi \in L^{\prime}(V)$. $L^{\prime}$ é equivalente a $L$ se $L \leq L^{\prime}$ e $L^{\prime} \leq L$; em símbolos, $L \equiv L^{\prime}$.

Teorema 3.1.5 Seja $L$ uma lógica com $L 1 \leq L$ e tendo as propriedades da Compacidade (1.1.15) e Löwenheim-Skolem para baixo (1.1.17). Então $L \equiv L 1{ }^{1}$

Prova. A idéia é supor por absurdo que existe $\psi \in L(V)$ que não é equivalente a uma sentença de primeira ordem. Por um argumento de Compacidade, vamos mostrar que a noção de satisfabilidade de $\psi$ depende apenas de um subconjunto finito $V_{0}$ de $V$. Depois, por um outro argumento de Compacidade, vamos construir dois modelos, um satisfazendo $\psi$ e o outro $\neg \psi$, mas de tal forma que sejam $L 1\left(V_{0}\right)$-equivalentes (a importância de tomar um $V_{0}$ finito vem de que neste passo fazemos uma enumeração das sentenças). Mas, por Löwenheim-Skolem, podemos assumir ainda que eles são enumeráveis; adicionaremos então condições aos modelos que permitirão construir um back-and-forth system (veja Hodges (1993)) entre os dois. Mas então, do teorema de que modelos enumeráveis parcialmente isomorfos são isomorfos (Hodges (1993)), chegaremos a uma contradição com 1.1.2.

Afirmação: Dado $\psi \in L(V)$, existe $V_{0} \subseteq_{f} V$ (isto é, $V_{0}$ está contido em $V$ e é finito) tal que a satisfabilidade de $\psi$ só depende de $V_{0}$. Mais precisamente,

$$
M \uparrow V_{0} \cong N \uparrow V_{0} \Rightarrow(M \vDash \psi \Leftrightarrow N \vDash \psi) .
$$

De fato, seja $\Gamma \subseteq L\left(V \cup V^{\prime}\right)$, onde $V^{\prime}$ é um renomeamento disjunto de $V$, o seguinte conjunto:

$$
\begin{aligned}
\Gamma= & \left\{\forall x_{1} \ldots \forall x_{n}\left(R x_{1} \ldots x_{n} \leftrightarrow R^{\prime} x_{1} \ldots x_{n}\right): n \geq 1, R n \text {-ária } \in V\right\} \\
& \cup\left\{\forall x_{1} \ldots \forall x_{n}\left(f\left(x_{1}, \ldots, x_{n}\right)=f^{\prime}\left(x_{1}, \ldots, x_{n}\right): n \geq 1, f n \text {-ária } \in V\right\}\right. \\
& \cup\left\{c=c^{\prime}: c \text { é símbolo de constante } \in V\right\}
\end{aligned}
$$

Temos que $\Gamma \vDash \psi \leftrightarrow \psi^{\prime}$. Logo, existe $\Gamma_{0} \subseteq_{f} \Gamma$ tal que $\Gamma_{0} \vDash \psi \leftrightarrow \psi^{\prime}$, pois se para todo $\Gamma_{0} \subseteq_{f} \Gamma$ existe uma estrutura $M \operatorname{com} M \vDash \Gamma_{0}$ e $M \not \models \psi \leftrightarrow \psi^{\prime}$, então cada parte finita de $\Gamma \cup\left\{\neg\left(\psi \leftrightarrow \psi^{\prime}\right)\right\}$ tem modelo, e assim por Compacidade tem modelo, uma contradição.

Assuma agora que $L 1$ não é tão forte quanto $L$, isto é, que existe $\psi \in L(V)$ não equivalente a uma sentença de primeira ordem. Tome $V_{0} \subseteq_{f} V$ tal que a satisfabilidade de $\psi$ só dependa de $V_{0}$. Considere $\varphi_{n}, n \geq 1$, uma enumeração de $L 1\left(V_{0}\right)$. Vamos construir uma sequencia $\psi_{n}, n \geq 1$, tal que para cada $n, \psi \wedge \psi_{1} \wedge \ldots \wedge \psi_{n}$ não é equivalente a uma sentença de primeira ordem; vai seguir que $\psi \wedge \psi_{1} \wedge \ldots \wedge \psi_{n}$ e $(\neg \psi) \wedge \psi_{1} \wedge \ldots \wedge \psi_{n}$ são satisfazíveis. A construção é por indução; o passo $n=1$ será suficiente. Veja que $\psi$ tem modelo, pois se não é equivalente a $\varphi \wedge \neg \varphi$ para qualquer $\varphi$ de primeira ordem. Semelhantemente, $\neg \psi$ também tem modelo. Agora se $\varphi\left(=\varphi_{1}\right)$ é tal que $\operatorname{Mod}(\varphi)=\operatorname{Mod}$, coloque então $\psi_{1}=\varphi$, pois como $\operatorname{Mod}(\psi \wedge \varphi)=\operatorname{Mod}(\psi)$, temos o que queríamos. Se $\operatorname{Mod}(\varphi)=\emptyset$, então $\psi_{1}=\neg \varphi$. Se ambos $\varphi$ e $\neg \varphi$ tem modelo, suponha por absurdo que $\psi \wedge \varphi$ e $\psi \wedge \neg \varphi$ são ambos equivalentes a fórmulas de primeira ordem, digamos a $\sigma$ e a $\chi$, respectivamente. Então, como $L$ satisfaz 3.1.3, valem as "leis de Boole", e assim

$$
\begin{aligned}
(\psi \wedge \varphi) & \vee(\psi \wedge \neg \varphi) \equiv \sigma \vee \chi \\
((\psi \wedge \varphi) \vee \psi) & \wedge((\psi \wedge \varphi) \vee \neg \varphi) \equiv \sigma \vee \chi
\end{aligned}
$$

\footnotetext{
${ }^{1}$ Essa é uma versão do teorema de Lindström; o original é um pouco diferente (Lindström (1969)). Veja também que não usaremos todo o poder de Löwenheim-Skolem para baixo: basta que seja válido para sentenças.
} 


$$
\begin{gathered}
\psi \wedge(\varphi \vee \neg \varphi) \equiv \sigma \vee \chi \\
\psi \equiv \sigma \vee \chi
\end{gathered}
$$

absurdo com a hipótese sobre $\psi$. Tome então $\psi_{1}$ como desejado. Veja que $(\neg \psi) \wedge \psi_{1}$ é satisfazível, caso contrário $\psi \vee\left(\neg \psi_{1}\right) \equiv \sigma \vee \neg \sigma$, donde $\left(\psi \vee\left(\neg \psi_{1}\right)\right) \wedge \psi_{1} \equiv \psi_{1}$, e assim $\psi \wedge \psi_{1} \equiv \psi_{1}$, um absurdo.

Seja agora $\Psi=\left\{\psi_{n}, n \geq 1\right\}$. Por Compacidade e Löwenheim-Skolem para $L$, existem estruturas enumeráveis $M$ e $N$ tais que $M \vDash \Psi \cup\{\psi\}$ e $N \vDash \Psi \cup\{\neg \psi\}$. Mas então $M \uparrow$ $V_{0} \equiv_{L 1} N \uparrow V_{0}$, e como a satisfabilidade de $\psi$ depende de $V_{0}, M \uparrow V_{0} ¥ N \uparrow V_{0}$. Além disso, $|M|$ e $|N|$ são infinitos enumeráveis, e assim podemos assumir que $|M|=|N|$.

O que vamos fazer agora é mostrar que podemos tomar $M \uparrow V_{0} \cong N \uparrow V_{0}$, obtendo uma contradição. Cosidere $V^{\prime}$ um renomeamento disjunto de $V$ e novos símbolos de funções $(2 n+1)$-árias $\left\{f_{n}, g_{n}: n \in \omega\right\}$. Seja $V^{*}=V \cup V^{\prime} \cup\left\{f_{n}, g_{n}: n \in \omega\right\}$. Para cada $n \in \omega$, enumere as fórmulas $\chi$ de $L 1\left(V_{0}\right)$ tais que $V L(\chi) \subseteq\left\{x_{1}, \ldots, x_{n}\right\}$, e considere o seguinte conjunto $\Gamma$ de sentenças de $L\left(V^{*}\right)$, que servirão para construir o isomorfismo desejado (sejam $\bar{x}=x_{1}, \ldots, x_{n}$ e $\left.\bar{y}=y_{1}, \ldots, y_{n}\right)$ :

$$
\begin{aligned}
& \forall \bar{x} \forall \bar{y} \forall x\left(\exists y\left(\bigwedge_{i=0}^{r}\left(\chi_{i}(\bar{x}, x) \leftrightarrow \chi_{i}^{\prime}(\bar{y}, y)\right)\right) \rightarrow \bigwedge_{i=0}^{r}\left(\chi_{i}(\bar{x}, x) \leftrightarrow \chi_{i}^{\prime}\left(\bar{y}, f_{n}(\bar{x}, \bar{y}, x)\right)\right)\right), \\
& \forall \bar{x} \forall \bar{y} \forall x\left(\exists y\left(\bigwedge_{i=0}^{r}\left(\chi_{i}(\bar{x}, x) \leftrightarrow \chi_{i}^{\prime}(\bar{y}, y)\right)\right) \rightarrow \bigwedge_{i=0}^{r}\left(\chi_{i}\left(\bar{x}, g_{n}(\bar{x}, \bar{y}, y)\right) \leftrightarrow \chi_{i}^{\prime}(\bar{y}, y)\right),\right.
\end{aligned}
$$

$n, r \in \omega$. Note que dado um subconjunto $\Gamma_{0}$ finito destas sentenças, podemos expandir uma $\left(V_{0} \cup V_{0}^{\prime}\right)$-estrutura qualquer a um modelo de $\Gamma_{0}$. Veja também que existe uma $\left(V_{0} \cup\right.$ $\left.V_{0}^{\prime}\right)$-estrutura satisfazendo

$$
\begin{gathered}
\psi, \neg \psi^{\prime} \\
\varphi \leftrightarrow \varphi^{\prime} \text {, para cada } \varphi \in L 1\left(V_{0} \cup V_{0}^{\prime}\right) .
\end{gathered}
$$

Para ver isto, tome $M$ um $V_{0}$-modelo de todas estas fórmulas exceto $\neg \psi^{\prime}$ (já vimos que existe tal $M$ ), e $N$ uma $V_{0}^{\prime}$-estrutura análoga, mas satisfazendo $\neg \psi^{\prime}$ ao invés de $\psi$. Defina agora uma $\left(V_{0} \cup V_{0}^{\prime}\right)$-estrutura $A$ assim: $|A|=|M|=|N|$, a interpretação do vocabulário $V_{0}$ é como em $M$, e a de $V_{0}^{\prime}$ como em $N$. Então $A\left\lceil V_{0}=M\right.$ e assim $A \vDash \psi$. Semelhantemente, temos $A \vDash \neg \psi^{\prime}$. Assim, por Compacidade e Löwenheim-Skolem existe um modelo enumerável $A$ de $\Gamma$. Sejam agora $M=A\left\lceil V\right.$ e $N=\left(A\left\lceil V^{\prime}\right)^{-1}\right.$. Então $|A|=|M|=|N|, M \vDash \psi, N \vDash \neg \psi$ e além disso $M \uparrow V_{0} \equiv_{L 1} N \uparrow V_{0}$. Vamos mostrar agora que $M \uparrow V_{0} \cong N \uparrow V_{0}$.

Seja $d_{n}, n \geq 1$, uma enumeração de $|A|$. Como $M \uparrow V_{0} \equiv_{L 1} N \uparrow V_{0}$, temos

$$
\begin{gathered}
\left\langle M \uparrow V_{0}, d_{1}\right\rangle \equiv_{L 1}\left\langle N \uparrow V_{0}, f_{0}\left(d_{1}\right)\right\rangle \\
\left\langle M \uparrow V_{0}, d_{1}, g_{1}\left(d_{1}, f_{0}\left(d_{1}\right), d_{1}\right)\right\rangle \equiv_{L 1}\left\langle N \uparrow V_{0}, f_{0}\left(d_{1}\right), d_{1}\right\rangle \\
\left.\left\langle M \uparrow V_{0}, d_{1}, g_{1}\left(d_{1}, f_{0}\left(d_{1}\right), d_{1}\right), d_{2}\right\rangle \equiv_{L 1}\left\langle N \uparrow V_{0}, f_{0}\left(d_{1}\right), d_{1}, f_{1}(\ldots), \ldots\right)\right\rangle
\end{gathered}
$$

Continuando assim obtemos sequencias $a_{1}, a_{2}, \ldots$ e $b_{1}, b_{2}, \ldots$ tais que $|M|=\left\{a_{n}: n \in \omega\right\}$, $|N|=\left\{b_{n}: n \in \omega\right\} \mathrm{e}$

$$
\left\langle M \uparrow V_{0}, a_{1}, a_{2}, \ldots\right\rangle \equiv_{L 1}\left\langle N \uparrow V_{0}, b_{1}, b_{2}, \ldots\right\rangle
$$

Mas então $f: M \uparrow V_{0} \cong N \uparrow V_{0}$, dado por $f\left(a_{n}\right)=b_{n}$. 


\subsection{O Número de Hanf de $L 2$}

Como visto na seção 1.2, a Semântica Padrão não satisfaz as propriedades de LöwenheimSkolem. No entanto, análogos dessas propriedades são satisfeitas por lógicas arbitrárias. Considere as seguintes definições:

Definição 3.2.1 Suponha que $\kappa$ seja um cardinal, que a lógica L contenha L1 (isto é, que $L 1 \leq L$, def. 3.1.4) e que $L(V)$ admita as mesmas estruturas que $L 1(V)$. Então

(a) O número de Löwenheim de $L(V)$ é o menor cardinal $\kappa$ tal que para toda $\varphi \in L(V)$ se $\varphi$ tem modelo, então tem modelo de cardinalidade menor ou igual a $\kappa$.

(b) O número de Hanf de $L(V)$ é o menor $\kappa$ tal que para toda $\varphi \in L(V)$ se $\varphi$ que tem modelo de cardinalidade $\kappa$, então tem modelo de cardinalidade arbitrariamente grande.

Assim, no caso de $L 1(V)$, os números de Löwenheim e de Hanf são $\aleph_{0}$. É claro que estas noções podem ser generalizadas de uma fórmula $\varphi$ para uma coleção de fórmulas $\Gamma$, e o teorema a seguir também será válido desde que $\Gamma$ forme um conjunto.

Proposição 3.2.2 Existem os números de Löwenheim e Hanf de L2.

Prova. Para cada $\varphi \in L 2$ satisfazível, seja

$$
\mathscr{L}(\varphi)=\text { menor cardinal do menor modelo de } \varphi
$$

e, se existe um limite para a cardinalidade dos modelos de $\varphi$, seja

$$
\mathscr{H}(\varphi)=\text { menor limite superior das cardinalidades dos modelos de } \varphi \text {. }
$$

Então o número de Löwenheim é o menor limite superior de $\{\mathscr{L}(\varphi): \varphi \in L 2\}$ e o número de Hanf é o menor cardinal estritamente maior que todo elemento em $\{\mathscr{H}(\varphi): \varphi \in L 2\}$.

No entanto, esta prova não fornece muita informação, além daquela que está no enunciado. Não temos, por exemplo, nenhuma estimativa sobre o tamanho do número de Hanf de $L 2$.

Observe também que na demonstração foi feito uso do axioma da substituição. O que vamos mostrar agora é que não é possível provar a existência do número de Hanf de $L 2$ sem fazer uso de alguma instância "vaga" deste axioma, no seguinte sentido: o axioma da substituição tem a forma

$$
(*) \forall y[\forall x(x \in y \rightarrow \exists z \varphi) \rightarrow \exists w \forall x(x \in y \rightarrow \exists z(z \in w \wedge \varphi))]
$$

onde $\varphi$ é qualquer fórmula de $L 1(\epsilon,=)$ onde $w$ não ocorra livre. Se $\varphi$ for uma fórmula que possua uma sub-fórmula do tipo $\forall x \psi$, com $x$ ocorrendo livre em $\psi$, então o axioma vai garantir a existência de um conjunto $w$ que, no entanto, supostamente já está dado, uma vez que o quantificador $\forall x$ percorre o universo dos conjuntos, e dessa forma temos uma espécie de argumento circular. Uma discussão sobre a formação de conceitos assim pode ser encontrada em Fraenkel et al. (1973). A exposição a seguir é baseada em Barwise (1972).

Para as considerações desta seção, será útil definir a Hierarquia Aritmética das fórmulas:

Definição 3.2.3 Uma fórmula está em (ou é) $\Sigma_{0}$, ou $\Pi_{0}$, ou $\Delta_{0}$, se for uma fórmula atômica ou se for da forma $\exists x(x \in y \wedge \psi)$ ou da forma $\forall x(x \in y \rightarrow \psi)$, com $\psi \in \Delta_{0}$. Ela estará em (ou será) $\Sigma_{n+1}$ se for $\Pi_{n}$ ou da forma $\exists x_{1} \ldots \exists x_{m} \psi$, com $\psi \in \Pi_{n}$ e estará em (ou será) $\Pi_{n+1}$ se for $\Sigma_{n}$ ou da forma $\forall x_{1} \ldots \forall x_{m} \psi$, com $\psi \in \Sigma_{n}$. 
Vamos considerar até o final da seção uma teoria de conjuntos de primeira ordem que chamaremos de $Z F_{1}$, que é a teoria obtida por se retirar todas as instâncias do axioma da substituição de $Z F$, com a exceção daquelas nas quais a fórmula $\varphi$ em (*) for $\Sigma_{1}$. Suporemos também que $L 1$ e $L 2$ estão formalizados dentro de $Z F_{1}$.

Lema 3.2.4 $Z F_{1}$ prova que se $\kappa$ é o número de Hanf de L2 e se $\varphi \in L 2$ tem, para cada cardinal $\lambda<\kappa$, um modelo de cardinalidade maior ou igual a $\lambda$, então $\varphi$ tem modelos de cardinalidade arbitrariamente grande.

Prova. Considere a sentença $\exists R \psi$, onde $\psi$ é

" $R$ é uma boa ordem e $\forall x \exists y(x R y \wedge \varphi$ se verifica relativizada a $\{z: z R y\})$ ".

Lembremos que pela observação $1.2 .8, \varphi$ pode ser tomada sem símbolos de constante, relação ou função, de tal forma que modelos de $\varphi$ são apenas conjuntos arbitrários. Suponha então que para todo $\lambda<\kappa$ exista um modelo $M_{\lambda}$ de $\varphi$ com cardinalidade maior ou igual a $\lambda$. Se algum $M_{\lambda}$ tem cardinalidade maior ou igual a $\kappa$, como $\kappa$ é número de Hanf, $\varphi$ tem modelos arbitrariamente grandes. Se não, por $\Sigma_{1}$-substituição, existe $\left\{M_{\lambda}: \lambda<\kappa\right\}$, e consequentemente existe $M=\bigcup_{\lambda<\kappa} M_{\lambda}$, que tem cardinalidade $\kappa$. Como cada $M_{\lambda}$ é um conjunto arbitrário, podemos tomar $M=\kappa$. Então, dado $x \in M$, existe $\lambda<\kappa$ tal que $x \in M_{\lambda}$; mas $M_{\lambda} \vDash \varphi$ e $M_{\lambda} \in M$, donde $M \vDash \exists R \varphi$. Mas $\kappa$ é número de Hanf; logo $\exists R \psi$ tem modelos arbitrariamente grandes, e consequentemente $\varphi$ também tem.

Corolário 3.2.5 O número de Hanf de L2 é um cardinal limite.

Prova. Suponha que seja da forma $\aleph_{\alpha+1}$ e $\operatorname{sejam~} M, \varphi$ tais que $M \vDash \varphi$ e que $M$ tenha cardinalidade $\aleph_{\alpha}$. Aplicando o lema, $\varphi$ tem que ter modelos arbitrariamente grandes; mas então $\aleph_{\alpha}$ tem que ser o número de Hanf, absurdo.

Definição 3.2.6 Um cardinal $\lambda$ é uniformemente $\kappa$-definível se existe uma fórmula $\Theta \in$ $L 1(\in,=)$ com uma única variável livre tal que para todo $\beta \geq \kappa$,

$$
\lambda=\text { único } a \in V_{\beta} \text { tal que } V_{\beta} \vDash \Theta[a] \text {, }
$$

onde $V_{\beta}$ é o mesmo conjunto do teorema 2.1.11 (observe que $V_{\beta}$ pode ser construído em $Z F_{1}$, já que $V_{\beta}=\bigcup_{\alpha<\beta} P\left(V_{\alpha}\right)$ ), que deve ser entendido como uma $\in$-estrutura.

Lema 3.2.7 $Z F_{1}$ prova que se $\kappa$ é o número de Hanf de L2 então para todo $\alpha<\kappa$ existe um cardinal $\lambda$ uniformemente $\kappa$-definivel tal que $\alpha<\lambda<\kappa$.

Prova. Observe primeiro que existe $\varphi \in L 2$ com modelo de cardinalidade maior que $\alpha$ mas sem modelos de cardinalidade maior ou igual a $\kappa$ : de fato, se não fosse assim, os modelos de toda $\varphi \in L 2$ seriam menores ou iguais a $\alpha$ ou $\varphi$ teria modelo de cardinalidade maior ou igual a $\kappa$; mas então qualquer cardinal $\lambda$ tal que $\alpha<\lambda<\kappa$ teria a propriedade de que se $\varphi$ tem modelos de cardinalidade maior ou igual a $\lambda$, então tem modelos de tamanho arbitrariamente grande, contrariando a definição de $\kappa$. Tome então $\lambda$ o menor cardinal tal que $\varphi$ não tem modelo de cardinalidade maior ou igual a $\lambda$ e seja $\Theta(\lambda)$ uma fórmula em $L 1(\epsilon,=)$ que expresse isto. Então $\Theta(x)$ é uma definição $\kappa$-uniforme de $\lambda$.

Lema 3.2.8 $Z F_{1}$ prova que se $\kappa$ é o número de Hanf de L2 e se $\varphi \in L 1(V)$ com $V_{\kappa} \vDash \varphi$, então $\forall \alpha \exists \beta\left(\beta \geq \alpha \wedge V_{\beta} \vDash \varphi\right)$. 
Prova. Seja $\psi$ a conjunção das seguintes sentenças:

$$
\begin{array}{cc}
\psi_{0}: & \forall X[\exists x X x \rightarrow \exists x(X x \wedge \neg \exists y(X y \wedge y \in x))] \\
\psi_{1}: & \forall x \forall y[\forall z(z \in x \leftrightarrow z \in y) \rightarrow x=y] \\
\psi_{2}: & \forall x \exists y \forall z[z \in y \leftrightarrow \forall w(w \in z \rightarrow w \in x)] \\
\psi_{3}: & \forall X \forall x \exists y \forall z(z \in y \leftrightarrow z \in x \wedge X x)
\end{array}
$$

(a interpretação do $\in$ é bem-fundada) (extensionalidade)

(partes)

(especificação de segunda ordem)

Note primeiro que, pelo argumento da proposição 2.1.5, se $M \vDash \psi_{0} \wedge \psi_{1}$, então $M$ é isomorfo a um $\in$-modelo transitivo e bem-fundado. Então, se $M \vDash \psi$, pelo argumento do teorema 2.1.10, $M$ é supertransitivo e, por 2.1.11, é isomorfo a $V_{\beta}$, para $\beta=O N^{M}$ ordinal limite. Note ainda que se $\beta$ é um ordinal limite qualquer, então $V_{\beta} \vDash \psi$, isto é,

$$
\text { (*) } M \vDash \psi \Leftrightarrow \exists \beta \text { limite tal que } M \cong V_{\beta} \text {. }
$$

Considere agora a sentença $\varphi \wedge \psi$; como $\kappa$ é número de Hanf e $V_{\kappa} \vDash \varphi \wedge \psi, \varphi \wedge \psi$ tem modelos arbitrariamente grandes; mas por $(*)$ estes modelos tem que ser da forma $V_{\beta}$.

Teorema 3.2.9 $Z F_{1}$ prova que se $\kappa$ é o número de Hanf de $L 2$, então $V_{\kappa} \vDash Z F_{1}$.

Prova. Como $\kappa$ é limite, $\kappa \geq \omega$. Mas, pelo último lema, $\kappa \neq \omega$, já que $V_{\omega} \vDash \neg \exists x(x=\omega)$. Agora, para qualquer ordinal limite $\beta>\omega$ temos que $V_{\beta}$ satisfaz todos os axiomas de $Z F$ com a possível exceção do axioma da substituição e, assim, só precisamos mostrar que $V_{\kappa}$ é modelo de $\Sigma_{1}$-substituição. Seja $\psi(x, y, z)$ da forma $\exists x_{1} \ldots \exists x_{n} \chi(x, y, z)$, onde todos os quantificadores de $\chi$ são limitados e suponha que para certos $a, b \in V_{\kappa}$,

$$
V_{\kappa} \vDash \forall x(x \in a \rightarrow \exists y \psi(x, y, b)),
$$

mas que

$$
V_{\kappa} \vDash \neg \exists w \forall x(x \in a \rightarrow \exists y(y \in w \wedge \psi(x, y, b))) .
$$

(o caso em que $\psi$ tem mais variáveis segue deste usando pares ordenados). Seja $\alpha<\kappa$ tal que $a, b \in V_{\alpha}$ e use o lema 3.2.7 para tomar um $\lambda$ tal que $\alpha<\lambda<\kappa$ e uma $\kappa$-definição uniforme $\Theta(x)$ de $\lambda$. A seguinte sentença é válida em $V_{\kappa}$, e consequentemente em $V_{\beta}$ para $\beta$ arbitrariamente grande:

$$
\exists z\left(\Theta(z) \wedge \exists u, v \in V_{z}(\forall x \in u \exists y \psi(x, y, v) \wedge \neg \exists w \forall x \in u \exists y \in w \psi(x, y, v))\right) .
$$

Assim, para $\beta \geq \kappa$ arbitrariamente grande, existem $a, b \in V_{\beta}$ tais que

$$
V_{\beta} \vDash \forall x \in a \exists y \psi(x, y, b) \wedge \neg \exists w \forall x \in a \exists y \in w \psi(x, y, b),
$$

que é equivalente a uma fórmula $Q(\beta, a, b) \in \Sigma_{1}$, já que o predicado " $M$ satisfaz $\varphi$ com a atribuição $s$ " é um predicado $\Sigma_{1}$. Por separação, defina $X=\left\{\langle a, b\rangle \in V_{\lambda}^{2}: \exists \beta Q(\beta, a, b)\right\}$. Para todo $\langle a, b\rangle \in X$ existe o menor $\beta$ tal que $Q(\beta, a, b)$; assim, por $\Sigma_{1}$-substituição, existe $\gamma$ maior que todos estes $\beta$. Mas como $\exists a \exists b Q(\beta, a, b)$ se verifica para $\beta$ arbitrariamente grande, para algum $\beta_{1}>\gamma$ e certos $a, b \in V_{\lambda}$ temos $Q\left(\beta_{1}, a, b\right)$. Mas então, por definição de $\gamma$, para algum $\beta_{0}<\gamma$ temos $Q\left(\beta_{0}, a, b\right)$. Logo

$$
V_{\beta_{0}} \vDash \forall x(x \in a \rightarrow \exists y \psi(x, y, b))
$$

e, consequentemente, 


$$
V_{\beta_{1}} \vDash \forall x\left(x \in a \rightarrow \exists y\left(y \in V_{\beta_{0}} \wedge \psi(x, y, b)\right)\right) .
$$

Mas isto contradiz $Q\left(\beta_{1}, a, b\right)$, tomando $w=V_{\beta_{0}}$.

Corolário 3.2.10 Se $Z F_{1}$ é consistente, então $Z F_{1}$ não prova que existe o número de Hanf de $L 2$.

Qual seria o tamanho do número de Hanf de $L 2$ ? Observe que " $\kappa$ é o número de Hanf de L2" pode ser escrita na forma

$\exists \kappa\left(\kappa=\mu \sigma\left(\forall \varphi \in L 2\left(\exists M, M^{\prime} \forall \gamma \geq \sigma\left(M \vDash \varphi \wedge \sigma \leq \operatorname{Card}(M) \rightarrow \gamma \leq \operatorname{Card}\left(M^{\prime}\right) \wedge M^{\prime} \vDash \varphi\right)\right)\right)\right)$,

que é uma fórmula $\Sigma_{3}$, já que $\operatorname{Card}(x)$ é $\Sigma_{1}$. Será que algum axioma do tipo "existem cardinais grandes, mensuráveis e inacessíveis arbitrariamente grandes" ou "existem cardinais grandes fortemente compactos arbitrariamente grandes" garantiriam a existência do número de Hanf de $L 2$ ? A resposta é não, pelo próximo teorema, já que estas sentenças são $\Pi_{2}$ e $\Pi_{3}$, respectivamente:

Teorema 3.2.11 Seja A uma sentença $\Pi_{3}$. Se

$$
Z F_{1} \vdash A \rightarrow \exists x(x \text { é o número de Hanf de L2), }
$$

então $Z F \vdash \neg A$.

Prova. Seja $\mathrm{Val}_{3}(x)$ a fórmula $\Sigma_{3}$ de uma variável livre que define validade para sentenças $\Sigma_{3}$ (Lévy (1965)).

Lema 3.2.12 ZF prova que se $\kappa$ é o número de Hanf de L2 e $\sigma$ é uma sentença $\Sigma_{3}$ tal que $V_{\kappa} \vDash \sigma$, então $\operatorname{Val}_{3}(\sigma)$.

Prova. Se $V_{\kappa} \vDash \exists x \forall y \exists z \psi(x, y, z)$, com $\psi \in \Delta_{0}$, seja $\alpha<\kappa$ tal que $a \in V_{\alpha}$ e $V_{\kappa} \vDash$ $\forall y \exists z \psi(a, y, z)$. Tome $\alpha<\lambda<\kappa$ e $\Theta(x)$ uma $\kappa$-definição uniforme de $\lambda$; então a sentença

$$
\exists \lambda\left(\Theta(\lambda) \wedge a \in V_{\lambda}(\forall y \exists z \psi(a, y, z))\right)
$$

é válida em $V_{\kappa}$, e consequentemente em $V_{\beta}$ para $\beta$ arbitrariamente grande. Considere agora $X=\left\{a \in V_{\lambda}: \exists \beta\left(V_{\beta} \vDash \forall y \exists z \psi(a, y, z)\right)\right\}$ e suponha por absurdo que

$$
\forall a \in X \exists \beta^{\prime}\left(\forall \beta\left(V_{\beta} \vDash \forall y \exists z \psi(a, y, z) \rightarrow \beta \leq \beta^{\prime}\right)\right) ;
$$

como estamos em $Z F$, podemos aplicar substituição e conseguir um $\gamma$ maior do que todos estes $\beta^{\prime}$; mas isto contraria o fato de que $\exists a\left(V_{\beta} \vDash \forall y \exists z \psi(a, y, z)\right)$ se verifica para $\beta$ arbitrariamente grande, e consequentemente existe um $a \in V_{\lambda}$ tal que $V_{\beta} \vDash \forall y \exists z \psi(a, y, z)$ se verifica para $\beta$ arbitrariamente grande. Mas então $\forall y \exists z \psi(a, y, z)$ é válida, e portanto $\operatorname{Val}_{3}(\exists x \forall y \exists z \psi(x, y, z))$.

Seja agora $\forall x \exists y \forall z B \in \Pi_{3}, B \in \Delta_{0}$, e sejam também $\varphi$ a fórmula $\neg \forall x \exists y \forall z B$ e $\psi$ "existe $x$ o número de Hanf de $L 2$ "; assim a hipótese fica $Z F_{1} \vdash \neg \varphi \rightarrow \psi$. Seja $\kappa$ o número de Hanf de $L 2$; vamos mostrar agora que $V_{\kappa} \not \models \exists \alpha(\alpha$ é o número de Hanf de $L 2)$. Note primeiro que

$\varphi$ tem modelo de cardinalidade menor que $\kappa \Leftrightarrow \varphi$ tem um modelo que pertence a $V_{\kappa}$, 
pois se $M \vDash \varphi$ e $\operatorname{Card}(M)<\kappa$, como $M$ é apenas um conjunto, temos também que $\operatorname{Card}(M) \vDash \varphi$ e $\operatorname{Card}(M) \in V_{\kappa}$. Por outro lado, se $M \in V_{\kappa}$, então $\operatorname{Card}(M) \leq \operatorname{Card}^{V_{\kappa}}(M) \in$ $V_{\kappa}$, e assim $\operatorname{Card}(M)<\kappa$. Agora, suponha que $\left(\kappa_{0} \text { é o número de Hanf de } L 2\right)^{V_{\kappa}}$. Se $\varphi \in L 2$ é tal que $M \vDash \varphi$ e $M \in V_{\kappa}$ é tal que $\kappa_{0} \leq \operatorname{Card}(M) \leq \operatorname{Card}^{V_{\kappa}}(M)$, então $\forall \alpha \in V_{\kappa} \exists M^{\prime} \in V_{\kappa}, M^{\prime} \vDash \varphi$ tal que $\alpha \leq \operatorname{Card}^{V_{\kappa}}\left(M^{\prime}\right)$. Logo, para todo $\alpha \in V_{\kappa}$ tal que $\operatorname{Card}(\alpha)$, $\exists M^{\prime} \in V_{\kappa}, M^{\prime} \vDash \varphi$, tal que $\alpha \leq \operatorname{Card}^{V_{\kappa}}\left(M^{\prime}\right)$. Assim, como $\kappa=\sup \left\{\alpha \in V_{\kappa}: \operatorname{Ord}(\kappa)\right\}$, para todo cardinal $\lambda<\kappa$ existe $M$ tal que $\operatorname{Card}^{V_{\kappa}}(M) \geq \lambda$ e $M \vDash \varphi$. Note agora que $\operatorname{Card}(M)$ também será maior ou igual a $\lambda$ : de fato, $\lambda \leq \operatorname{Card}^{V_{\kappa}}\left(M^{\prime}\right)=\alpha \Rightarrow \lambda \leq \operatorname{Card}(\alpha)$. Seja $f: M^{\prime} \rightarrow \alpha$ uma bijeção entre $M^{\prime}$ e $\alpha$ (já que $\operatorname{Card}^{V_{\kappa}}\left(M^{\prime}\right)=\alpha$ ) e seja $g$ uma bijeção entre $\alpha$ e $\operatorname{Card}(\alpha)$. Então a composta de $f$ e $g$ é uma bijeção entre $M^{\prime}$ e $\operatorname{Card}(\alpha)$, donde $\operatorname{Card}\left(M^{\prime}\right)=\operatorname{Card}(\alpha)=\operatorname{Card}\left(\operatorname{Card}^{V_{\kappa}}\left(M^{\prime}\right)\right)$. Consequentemente, pelo lema 3.2.4, $\varphi$ tem modelos de tamanho arbitrariamente grandes; mas então $\kappa_{0}$ é o número de Hanf de $L 2$, um absurdo.

Agora pelo teorema 3.2.9, $V_{\kappa} \vDash Z F_{1}$, e pelo que fizemos acima $V_{\kappa} \not \models \psi$. Logo $V_{\kappa} \vDash \varphi$, e o último Lema acarreta que $\operatorname{Val}_{3}(\varphi)$.

Corolário 3.2.13 Seja $T$ uma extensão de $Z F_{1}$ consistente com $Z F$ e obtida por se adicionar sentenças $\Pi_{3}$ a $Z F_{1}$. Então $T$ não prova a existência do número de Hanf de L2.

Vemos assim que o número de Hanf de $L 2$ é realmente um cardinal muito grande. Agora vamos encarar a seguinte pergunta: é possível, como o é para muitas lógicas, construir uma função $F$ delimitadora para o número de Hanf de $L 2$ ?

Teorema 3.2.14 Suponha que $Q(\lambda, \mu) \in \Sigma_{1}$ seja uma fórmula que define uma função $F(\lambda)=\mu, \lambda, \mu$ cardinais. Então existe $\varphi \in L 2$ tal que para $\lambda$ arbitrariamente grande, $\varphi$ tem modelo de cardinalidade $\kappa>\lambda$ mas nenhum com $\lambda \leq \kappa \leq F(\lambda)$.

Prova. Suponha por um momento que

(*) Para cada cardinal $\lambda$, existe $\varphi \in L 2$ e $\lambda^{\prime} \geq \lambda$ tais que

$\exists M\left(M \vDash \varphi \wedge \lambda^{\prime}<\operatorname{Card}(M) \mathrm{e}\right.$

$\neg \exists M\left(M \vDash \varphi \wedge \lambda^{\prime} \leq \operatorname{Card}(M) \leq F\left(\lambda^{\prime}\right)\right)$.

Se isto for verdade, suponha por absurdo que não existe $\varphi$ como no enunciado; então, para toda $\varphi \in L 2$ existe o menor $\alpha$ tal que se $\alpha \leq \lambda$, então $\varphi$ não tem modelo maior que $\lambda$ ou $\varphi$ tem modelo entre $\lambda$ e $F(\lambda)$. Por substituição, existe $\gamma$ maior que todos estes $\alpha$; logo,

$$
\forall \varphi \in L 2 \forall \lambda \geq \gamma(\neg \exists M(M \vDash \varphi \wedge \operatorname{Card}(M)>\lambda) \vee \exists M(M \vDash \varphi \wedge \lambda \leq \operatorname{Card}(M) \leq F(\lambda))) \text {, }
$$

contrariando $(*)$. Veja porém que $(*)$ pode ser escrita como uma fórmula $\Pi_{2}$, sendo, portanto, $\Sigma_{3}$; consequentemente, para prová-la, pelo teorema 3.2.9 basta assumirmos sua negação (que é $\left.\Pi_{3}\right)$ e provar em $Z F_{1}$ que o número de Hanf de $L 2$ existe.

Suponha então a negação de (*); então, para algum cardinal $\lambda$, para toda $\varphi \in L 2$, se $\lambda^{\prime} \geq \lambda$, então $\varphi$ tem modelo com cardinal entre $\lambda^{\prime}$ e $F\left(\lambda^{\prime}\right)$ se, e somente se, $\varphi$ tem modelo com cardinal maior que $\lambda^{\prime}$. Seja $X=\{\varphi \in L 2: \varphi$ não tem modelos arbitrariamente grandes $\}$; então $\varphi \in X \Leftrightarrow \exists \lambda^{\prime} \geq \lambda$ tal que $\varphi$ não tem modelos com cardinal entre $\lambda^{\prime}$ e $F\left(\lambda^{\prime}\right)$. Para cada $\varphi \in X$, seja $g(\varphi)=$ menor $\lambda^{\prime}$ tal que $\lambda^{\prime} \geq \lambda$ e $\varphi$ não tem modelo de cardinalidade entre $\lambda^{\prime}$ e $F\left(\lambda^{\prime}\right)$. g é definida por uma fórmula $\Sigma_{1}$, e $\operatorname{assim} \kappa=\sup \{g(\varphi): \varphi \in X\}$ existe por $\Sigma_{1}$-substituição; mas se $\varphi$ tem modelo de cardinalidade $\kappa$, então $\varphi$ tem modelos de cardinalidade arbitrariamente grandes, e assim o número de Hanf de L2 existe. 


\subsection{Definição de Verdade e Reduções}

Na seção 1.2 vimos que o conjunto das fórmulas válidas de $L 2$ com a semântica padrão não é recursivo. Nesta seção este conjunto será observado com mais cuidado, e veremos como a validade de uma fórmula de ordem superior à segunda (com uma semântica análoga à semântica padrão de $L 2$ ) pode ser reduzida à validade de uma de primeira ordem nos modelos de uma teoria de segunda ordem.

Ao longo desta seção, considere, para cada conjunto de sentenças da lógica $L$ num vocabulário $V$ recursivamente enumerável, a função \# que associa a cada sequencia $S$ de símbolos de $L$ seu número de Gödel \#S. Os números naturais que sob esta codificação representam sequencias serão chamados de números-sequencia. Vamos começar notando que o conjunto das fórmulas de primeira ordem válidas da aritmética é definível por uma fórmula de segunda ordem.

Definição 3.3.1 Sejam $L, L^{\prime}$ lógicas arbitrárias e $\Gamma \subseteq L\left(V_{N}\right)$, onde $V_{N}$ é o vocabulário de $N$, a estrutura dos números naturais. Então, uma definição de verdade em $L^{\prime}$ para $\Gamma$ (sobre $N$ ) é uma fórmula $\tau$ de $L^{\prime}$ com uma única variável livre tal que $N \vDash \varphi \leftrightarrow \tau[\# \varphi]$, para toda $\varphi \in \Gamma$.

Teorema 3.3.2 Não existe uma definição de verdade em L1 para o conjunto das sentenças de primeira ordem válidas em $N$, mas existe uma em $L 2$.

Prova. O fato de que não existe uma definição de verdade em $L 1$ é uma consequencia do teorema da indefinibilidade da verdade de Tarski (Enderton (2000)). Para construir a definição de verdade em $L 2$, defina uma atribuição finita de valores como um númerosequencia que representa uma sequencia da forma

$$
\left\langle\left\langle x_{0}, n_{0}\right\rangle, \ldots,\left\langle x_{k}, n_{k}\right\rangle\right\rangle
$$

onde $x_{0}, \ldots, x_{k}$ são variáveis e $n_{0}, \ldots, n_{k}$ números naturais, e seja $\operatorname{Atrib}(x)$ o predicado " $x$ é uma atribuição finita". Considere também as seguintes funções recursivas, definidas para números-sequencia:

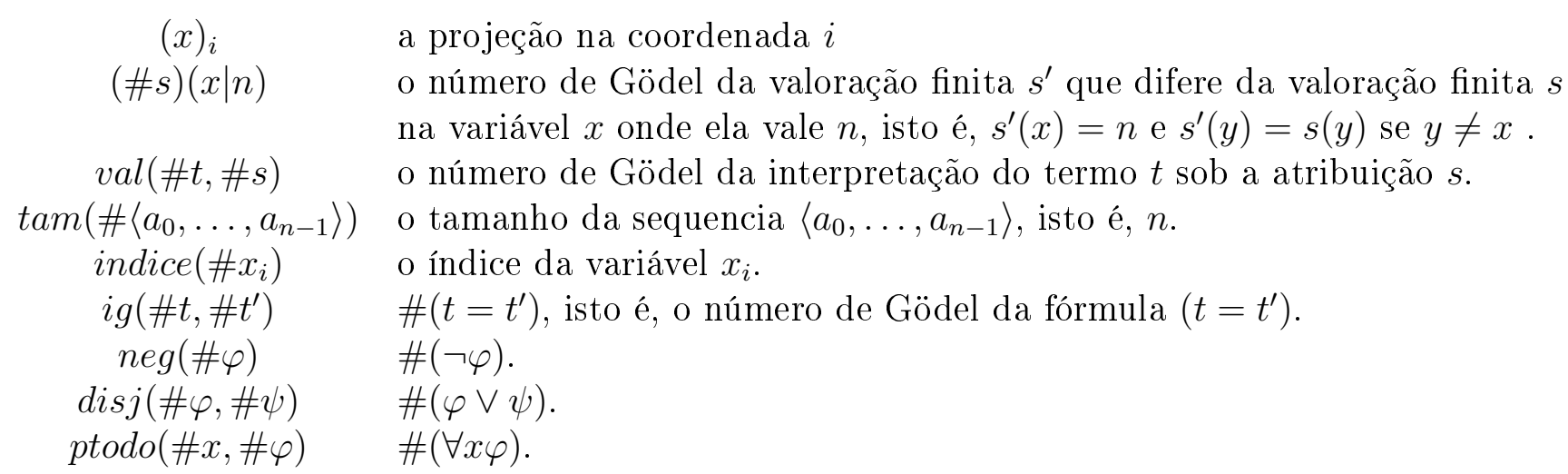

Para definir a validade de uma fórmula sob uma atribuição $s$, contudo, temos o problema de que a fórmula pode conter variáveis às quais $s$ não atribui valores, já que $s$ é finita. Definiremos então um predicado recursivo AtribVal $(\# \varphi, \# s)$, "s é uma atribução válida para $\varphi "$, como

$$
\operatorname{AtribVal}(x, y) \leftrightarrow \operatorname{Atrib}(y) \wedge \forall i<\operatorname{tam}(x)\left(\operatorname{var}\left((x)_{i}\right) \rightarrow \operatorname{indice}\left((x)_{i}\right)<\operatorname{tam}(y)\right),
$$


onde $\operatorname{var}(x)$ é o predicado " $x$ é o número de Gödel de uma variável". Vamos agora construir uma fórmula de segunda ordem $\tau_{0}$ com uma única variável livre de segunda ordem $X$ que garantirá que se $\varphi$ é válida sob a atribuição $s$, então $N \vDash \tau_{0}(X) \rightarrow X(x, y)[\# \varphi, \# s]$ :

$$
\begin{aligned}
\tau_{0}(X) \leftrightarrow \quad & \forall t, t^{\prime}, s\left(\operatorname{Term}(t) \wedge \operatorname{Term}\left(t^{\prime}\right) \wedge \operatorname{AtribVal}\left(i g\left(t, t^{\prime}\right) s\right) \rightarrow\right. \\
& \left(X\left(\operatorname{ig}\left(t, t^{\prime}\right), s\right) \leftrightarrow \operatorname{val}(t, s)=\operatorname{val}\left(t^{\prime}, s\right)\right) \\
& \wedge \forall u, s(\operatorname{Form}(u) \wedge \operatorname{AtribVal}(u, s) \rightarrow(X(\operatorname{neg}(u), s) \leftrightarrow \neg X(u, s))) \\
& \wedge \forall u, v, s(\operatorname{Form}(u) \wedge \operatorname{Form}(v) \wedge \operatorname{AtribVal}(\operatorname{disj}(u, v), s) \rightarrow \\
& (X(\operatorname{disj}(u, v), s) \leftrightarrow X(u, s) \vee X(v, s))) \\
& \forall u, x, s(\operatorname{Form}(u) \wedge \operatorname{var}(x) \wedge \operatorname{AtribVal}(u) \rightarrow(X(\operatorname{ptodo}(x, u), s) \leftrightarrow \forall n X(u, s(x \mid n)))) .
\end{aligned}
$$

Agora, a definição de $\tau_{0}$ usa um conjunto finito de funções recursivas; seja $D$ uma conjunção de fórmulas que definam estas funções e que use as variáveis de função $g_{1}, \ldots, g_{m}$ nestas definições e coloque

$$
\tau(x) \leftrightarrow \forall g_{1} \ldots g_{m}\left(D \rightarrow \forall X\left(\tau_{0}(X) \rightarrow X(x, 0)\right)\right) .
$$

Então $N \vDash \varphi \leftrightarrow \tau[\# \varphi]$ para toda sentença de primeira ordem $\varphi$.

Lógica de Ordem Finita. Vamos agora introduzir a Lógica de Ordem Finita, também chamada de $\omega$-Lógica, ou ainda de Teoria dos Tipos, e que vamos denotar por $L \omega$. Ela é uma extensão da Lógica de Segunda Ordem na qual permite-se quantificação sobre objetos de ordem arbitrária; assim, podemos enunciar, por exemplo, a existência de uma relação entre uma relação binária de segunda ordem e um elemento do domínio, o que seria então uma relação de terceira ordem. Nossa definição será a de uma variante relacional, isto é, sem variáveis para funções.

Definição 3.3.3 (a) Um tipo é uma sequencia finita gerada indutivamente do seguinte modo: ı é um tipo e se $\tau_{1}, \ldots, \tau_{k}$ são tipos, $k \geq 0$, então $\left(\tau_{1}, \ldots, \tau_{k}\right)$ é um tipo. O tipo () é denotado por o. O conjunto de todos os tipos é denotado por $\mathcal{T}$.

(b) $O$ conjunto das variáveis de $L \omega(V)$ é um conjunto infinito enumerável tal que para cada variável $X$ está associado um único tipo (chamado de tipo da variável), e para cada tipo existe uma quantidade infinita enumerável de variáveis associadas a este tipo. Semelhantemente, a cada símbolo de $V$ está associado um único tipo. Os termos de $L \omega(V)$ são as variáveis e símbolos de tipo $\iota$ e as fórmulas são construídas do modo usual, onde as fórmulas atômicas são $Z\left(P_{1}, \ldots, P_{n}\right)$, onde $Z$ é uma variável ou símbolo do tipo $\tau=\left(\tau_{1}, \ldots, \tau_{n}\right)$ e $P_{i}$ é uma variável ou símbolo de tipo $\tau_{i}, 1 \leq i \leq n$, e a quantificação é feita em cima de variáveis de qualquer tipo.

(c) A semântica padrão de Lu é definida de modo semelhante à de L2: se $M$ é um conjunto arbitrário, defina o conjunto $M_{\tau}$ dos objetos de tipo $\tau$ sobre $M$ do seguinte modo: $M_{\iota}=M$ e se $\tau=\left(\tau_{1}, \ldots, \tau_{k}\right)$, então $M_{\tau}=P\left(M_{\tau_{1}} \times \ldots \times M_{\tau_{k}}\right)$. A função de interpretação $I$ associa a símbolos de tipo $\tau$ objetos de tipo $\tau$. Uma atribuição s para L $\omega$ é então uma função definida no conjunto das variáveis tal que se $X$ é uma variável do tipo $\tau$, entãa $s(X) \in M_{\tau}{ }^{2}{ }^{2}$

(d) $A$ ordem od $(\tau)$ de um tipo $\tau \neq$ o é definida por indução: od $(\iota)=1, \operatorname{eod}\left(\tau_{1}, \ldots, \tau_{k}\right)=$ $\max \left\{\operatorname{od}\left(\tau_{1}\right), \ldots, \operatorname{od}\left(\tau_{k}\right)\right\}+1$. A ordem de uma variável é ordem de seu tipo e a ordem de $\varphi \in L \omega$ é a maior das ordens dos tipos das variáveis que ocorrem em $\varphi$. A lógica de n-ésima ordem é formada pelas fórmulas de ordem menor ou igual a n e uma atribuição de n-ésima ordem é uma atribuição de L $\omega$ restrita ao conjunto das variáveis de ordem menor ou igual a n (veja que desse modo recuperamos a variante relacional da Lógica de Segunda Ordem).

\footnotetext{
${ }^{2}$ Veja que desse modo $M_{o}=P(\{\emptyset\})=\{\emptyset,\{\emptyset\}\}$, e assim $M_{o}$ pode ser identificado com os valores verdade.
} 
Estamos agora em posição agora de estabelecer um análogo do Teorema 3.3.2:

Teorema 3.3.4 Não existe uma definição de verdade em L2 para o conjunto das sentenças de segunda ordem válidas em $N$, mas existe uma em $L 3$.

Prova. As diversas abreviações que serão utilizadas na construção das fórmulas abaixo estão descritas na prova do teorema 3.3.2. Vamos supor que todas as variáveis de segunda ordem são de relações unárias; nada é perdido pois sequencias finitas de elementos são codificadas em números-sequencia. Nós vamos codificar uma atribuição (finita) de segunda ordem num conjunto de números. Seja $S$ uma atribuição de segunda ordem com domínio $\left\{x_{1}, \ldots, x_{k}, X_{1}, \ldots, X_{l}\right\}$. Então $S$ pode ser codificada assim:

$$
\# S=\{\# s\} \cup \bigcup_{i=1}^{l}\left\{\# X_{i}\right\} \times S\left(X_{i}\right),
$$

onde abusamos da notação em \#S e $s$ é uma atribuição de primeira ordem de domínio $\left\{x_{1}, \ldots, x_{k}\right\}$ tal que $s\left(x_{i}\right)=S\left(x_{i}\right), 1 \leq i \leq k$, e $\{n\} \times A=\{\#\langle n, a\rangle: a \in A\}$. Defina os predicados $V A R(\# X)$, "X é uma variável de relação" e $A T R I B(\# S)$, "S é uma atribuição (finita) de segunda ordem":

$$
\operatorname{ATRIB}(X) \leftrightarrow \forall n(X(n) \rightarrow \operatorname{Atrib}(s) \vee \exists x \exists y(V A R(x) \wedge n=\#\langle x, y\rangle) .
$$

Defina também a função aplic $(\# V, \# t)$, que retorna \#V(t) e também o predicado $V A L(\# T, \# S)$, "a interpretação do termo ou variável de segunda ordem $T$ sob a atribuição $S$ ":

$$
\begin{gathered}
V A L(X, Y)(n) \leftrightarrow \quad A T R I B(Y) \wedge[(T e r m(X) \wedge \exists s(\operatorname{Atrib}(s) \wedge Y s \wedge \operatorname{val}(X, s)=n)) \\
\vee(V A R(X) \wedge \exists m(Y m \wedge m=\#\langle X, n\rangle))]
\end{gathered}
$$

De tal forma que se $t$ é um termo e $S$ é uma atribuição de segunda ordem, então $V A L^{N}(\# t, \# S)=$ $\left\{\right.$ val $\left.^{N}(\# t, \# s)\right\}$, onde $s$ é a atribuição de primeira ordem tal que $S \uparrow \operatorname{dom}(s)=s$ e, se $X$ é uma variável de relação, então $V A L^{N}(\# X, \# S)=S(X)$. Consideremos agora o predicado $A T R I B \operatorname{Val}(\# \varphi, \# S)$, " $S$ é uma atribuição válida para $\varphi$ ":

$$
\begin{aligned}
& \operatorname{ATRIBVal}(X, Y) \leftrightarrow A T R I B(Y) \wedge[\forall n(Y(n) \wedge \operatorname{Atrib}(n) \rightarrow \operatorname{AtribVal}(X, n)) \\
& \wedge \exists i<\operatorname{tam}(X)\left(\left(\operatorname{VAR}\left((X)_{i}\right) \rightarrow \exists m\left(\operatorname{Sm} \wedge(X)_{i}=(m)_{1}\right)\right)\right] .
\end{aligned}
$$

Defina agora a fórmula $\tau_{0}^{2}$ de tal forma que $N \vDash \tau_{0}(T) \rightarrow T(X, Y)[\{\# \varphi\}$, \#S], onde $T$ é uma variável de terceira ordem:

$$
\begin{aligned}
\tau_{0}^{2}(T) \leftrightarrow \quad \forall t, X, S(\operatorname{Term}(t) \wedge V A R(X) \wedge A T R I B V \operatorname{Val}(\operatorname{aplic}(X, t), S) \\
\rightarrow(T(\operatorname{aplic}(X, t), S) \leftrightarrow \exists n(V A L(t, S)(n) \wedge V A L(X, S)(n))) \\
\wedge \forall u, S(F \operatorname{Frm}(u) \wedge A T R I B V \operatorname{Val}(u, S) \rightarrow(T(\operatorname{neg}(u), S) \leftrightarrow \neg T(u, S))) \\
\wedge \forall u, v, S(\operatorname{Form}(u) \wedge F \operatorname{Frm}(v) \wedge A T R I B V a l(\operatorname{disj}(u, v), S) \\
\rightarrow(T(\operatorname{disj}(u, v), S) \leftrightarrow T(u, S) \vee T(v, S)) \\
\wedge \forall u, x, S(F \operatorname{Frm}(u) \wedge(\operatorname{var}(x) \vee V A R(x)) \wedge A T R I B V a l(p t o d o(x, u), S) \\
\rightarrow\left(T(\operatorname{ptodo}(x, u), S) \leftrightarrow \forall S^{\prime}\left(S^{\prime}=S \bmod (x) \rightarrow T\left(u, S^{\prime}\right)\right)\right)
\end{aligned}
$$

onde $S^{\prime}=S \bmod (x)$ abrevia

$$
\begin{gathered}
\operatorname{ATRIB}\left(S^{\prime}\right) \\
\wedge\left[\operatorname{var}(x) \rightarrow \forall n\left(\neg \operatorname{Atrib}(n) \rightarrow\left(\operatorname{Sn} \leftrightarrow S^{\prime} n\right)\right)\right. \\
\wedge \forall s, s^{\prime}\left(\operatorname{Atrib}(s) \wedge \operatorname{Atrib}\left(s^{\prime}\right) \wedge S(s) \wedge S^{\prime}\left(s^{\prime}\right)\right. \\
\left.\left.\rightarrow \operatorname{tam}\left(s^{\prime}\right)=\operatorname{tam}(s) \wedge \exists i<\operatorname{tam}(s)\left(\left(\left(s^{\prime}\right)_{i}\right)_{1}=x\right) \wedge \forall i<\operatorname{tam}(s)\left(\left(\left(s^{\prime}\right)_{i}\right)_{1} \neq x \rightarrow\left(s^{\prime}\right)_{i}=(s)_{i}\right)\right)\right] \\
\vee\left[V A R(X) \rightarrow \forall s\left(\operatorname{Atrib}(s) \wedge S(s) \rightarrow S^{\prime}(s)\right) \wedge\right. \\
\left.\forall Y, Z\left(X \neq Y \rightarrow\left((X \times Z) \subseteq S \leftrightarrow(Y \times Z) \subseteq S^{\prime}\right)\right)\right] .
\end{gathered}
$$


Logo, para toda sentença $\varphi$, temos $N \vDash \varphi \Leftrightarrow N \vDash \forall T\left(\tau_{0}^{2}(T) \rightarrow T(X, Y)\right)[\{\# \varphi\}, \emptyset]$. Terminamos análogamente a 3.3.2.

A leitura atenta da prova de 3.3.4 mostra que o resultado pode ser generalizado para outros níveis da linguagem; enunciaremos a generalização, omitindo a prova:

Teorema 3.3.5 Não existe uma definição de verdade em Ln para o conjunto das sentenças de Ln válidas sobre $N$, mas existe uma em $L(n+1)$. Também não existe uma definição de verdade de ordem finita para o conjunto das sentenças de ordem finita válidas sobre $N$.

Reduções. Há um modo de ver a Lógica de Ordem Finita como uma Teoria de Tipos de primeira ordem. Vamos examinar agora como isto pode ser feito. A primeira coisa a se notar é que a construção de modelos não-padrão feita para $L 2$ também pode ser feita para $L \omega:$

Definição 3.3.6 Uma pré-estrutura de Henkin $M^{H}$ para $L \omega(V)$ é uma coleção de conjuntos $M_{\tau}, \tau \in \mathcal{T}$, com $M_{\iota} \neq \emptyset$ satisfazendo $M_{\left(\tau_{1}, \ldots, \tau_{k}\right)} \subseteq P\left(M_{\tau_{1}} \times \ldots \times M_{\tau_{k}}\right)$ mais uma função de interpretação I tal que se $v \in V$ é do tipo $\tau$ então $I(v) \in M_{\tau}{ }^{3}$. Uma atribuição s para essa pré-estrutura é uma função que satisfaz $s(X) \in M_{\tau}$ para toda variável $X$ do tipo $\tau$.

Agora, a noção de validade de fórmulas nestas pré-estruturas pode ser reduzida à validade de fórmulas de primeira ordem num certo tipo de estruturas: suponha dado o vocabulário $V$ e, para cada tipo $\tau \neq o$, seja $D_{\tau}$ um novo símbolo de predicado unário e $\varepsilon_{\tau}$ um novo símbolo de predicado com aridade $o d(\tau)+1$. Vamos denotar o vocabulário criado a partir de $V$ mais estes símbolos novos por $V^{T}$.

Proposição 3.3.7 Seja $M^{H}$ uma pré-estrutura de Henkin para L $\omega(V)$. Então existe uma estrutura $M=\langle|M|, I\rangle$ de vocabulário $V^{T}$ satisfazendo

(1) para todos os simbolos $v$ de tipo $\tau, v^{M} \in D_{\tau}^{M}$,

(2) $D_{\tau} \neq \emptyset$ para todo $\tau$,

(3) se $\tau \neq \tau^{\prime}$, então $a \in D_{\tau}^{M} \Rightarrow a \notin D_{\tau^{\prime}}^{M}$,

(4) se $\tau=\left(\tau_{1}, \ldots, \tau_{k}\right)$, ent $\tilde{a} o\left\langle a, a_{1}, \ldots, a_{n}\right\rangle \in \varepsilon_{\tau}^{M} \Rightarrow a \in D_{\tau}^{M}$ e $a_{i} \in D_{\tau_{i}}^{M}, i \leq k$ e

(5) para todo $a \in M$, existe um tipo $\tau$ tal que $a \in D_{\tau}^{M}$.

Além disso, para toda sentença $\varphi \in L \omega(V)$,

$$
M^{H} \vDash \varphi \Leftrightarrow M \vDash \varphi^{T},
$$

onde $\varphi^{T}$ é a fórmula obtida a partir de $\varphi$ trocando-se toda ocorrência de fórmula atômica $Z\left(P_{1}, \ldots, P_{n}\right)$, onde $Z$ é uma variável ou símbolo do tipo $\tau=\left(\tau_{1}, \ldots, \tau_{k}\right), k \geq 1$ e $P_{i}$ é uma variável ou símbolo de tipo $\tau_{i}, i \leq k$, por $\varepsilon_{\tau}\left(Z, P_{1}, \ldots, P_{n}\right)$ e os quantificadores sobre variáveis de tipo $\tau$ são relativizados a $D_{\tau}$. Recíprocamente, para cada estrutura $M$ no vocabulário $V^{T}$ satisfazendo as propriedades (1) a (5), existe uma pré-estrutura de Henkin de L $\omega(V)$ para a qual a equivalência acima se verifica.

\footnotetext{
${ }^{3}$ Aqui não estamos seguindo rigorosamente a noção de modelo de Henkin do capítulo 1 já que lá não foi requerido que a interpretação dos símbolos de predicados $n$-ários estivessem nos $R_{n}^{H}$. Mas já que esta condição não é estranha e auxilia na obtenção de resultados mais adiante, decidimos exigi-la.
} 
Prova. Dada a pré-estrutura $M^{H}$, defina $M=\bigcup_{\tau \in \mathcal{T}} M_{\tau} \times\{\tau\}$ e $v^{M}=\left\langle v^{M^{H}}, \tau_{v}\right\rangle$ para cada símbolo $v \in V$, onde $\tau_{v}$ é o tipo de $v, D_{\tau}^{M}=\left\{x \in M: \pi_{2}(x)=\tau\right\}$ e, se $\tau=\left(\tau_{1}, \ldots, \tau_{k}\right)$, $k \geq 1$, então $\varepsilon_{\tau}^{M}=\left\{\left\langle a, a_{1}, \ldots, a_{k}\right\rangle \in M^{k+1}:\left\langle\pi_{1}\left(a_{1}\right), \ldots, \pi_{1}\left(a_{k}\right)\right\rangle \in \pi_{1}(a)\right\}$. Então é claro que (1) a $(5)$ e $(*)$ estão satisfeitos.

Recíprocamente, dado $M$ satisfazendo (1) a (5), defina $M_{\iota}=D_{\iota}^{M}$ e construa indutivamente uma família de funções $f_{\tau}$ e os conjuntos $D_{\tau}^{M}$ do seguinte modo: $f_{\iota}: D_{\iota}^{M} \rightarrow M_{\iota}$ é a identidade e, se $\tau=\left(\tau_{1}, \ldots, \tau_{k}\right)$, seja $f_{\tau}: D_{\tau} \rightarrow P\left(M_{\tau_{1}} \times \ldots \times M_{\tau_{k}}\right)$ uma função injetora tal que $\left\langle a, a_{1}, \ldots, a_{k}\right\rangle \in \varepsilon_{\tau}^{M} \Leftrightarrow\left\langle f_{\tau_{1}}\left(a_{1}\right), \ldots, f_{\tau_{k}}\left(a_{k}\right)\right\rangle \in f_{\tau}(a)$ e $M_{\tau}=\operatorname{Im}\left(f_{\tau}\right)$. Dado um símbolo $v \in V$ do tipo $\iota$, sua interpretação é definida como $I^{H}(v)=v^{M}$ e, se $\tau=\left(\tau_{1}, \ldots, \tau_{k}\right)$, então $I^{H}(v)=\left\{\left\langle f_{\tau_{1}}\left(a_{1}\right), \ldots, f_{\tau_{k}}\left(a_{k}\right)\right\rangle:\left\langle v^{M}, a_{1}, \ldots, a_{k}\right\rangle \in \varepsilon_{\tau}^{M}\right\}$.

Vamos provar $(*)$ por indução na complexidede de $\varphi$. Em primeiro lugar, veja que se $v$ é um símbolo do tipo $\tau$, então, por definição, $f_{\tau}\left(v^{M}\right)=I^{H}(v)$. Agora, se $\varphi$ for atômica, então é da forma $v\left(v_{1}, \ldots, v_{n}\right.$ ), onde $v, v_{1}, \ldots, v_{n}$ são símbolos (não podem ser variáveis pois $\varphi$ é sentença), $v$ é do tipo $\left(\tau_{1}, \ldots, \tau_{k}\right)$ e $v_{i}$ é do tipo $\tau_{i}, 1 \leq i \leq k$. Logo $M^{H} \vDash v\left(v_{1}, \ldots, v_{n}\right) \Leftrightarrow$ $\left\langle I^{H}\left(v_{1}\right), \ldots, I^{H}\left(v_{k}\right)\right\rangle \in I^{H}(v) \Leftrightarrow\left\langle f_{\tau_{1}}\left(v_{1}^{M}\right), \ldots, f_{\tau_{k}}\left(v_{k}^{M}\right)\right\rangle \in f_{\tau}\left(v^{M}\right) \Leftrightarrow\left\langle a, a_{1}, \ldots, a_{k}\right\rangle \in \varepsilon_{\tau}^{M} \Leftrightarrow$ $M \vDash \varepsilon_{\tau}\left(v, v_{1}, \ldots, v_{k}\right)$.

O caso dos conectivos proposicionais segue diretamente da hipótese de indução. Suponha então que $\varphi$ é da forma $\forall X^{\tau} \psi$; então $M^{H} \vDash \forall X^{\tau} \psi \Leftrightarrow$ para toda atribuição $s$ em $M^{H}$ temos $M^{H} \vDash \psi[s] \Leftrightarrow$ para toda atribuição $s$ em $M^{H}\left\langle M^{H}, s\left(X^{\tau}\right)\right\rangle \vDash \psi\left(X^{\tau} \mid Z\right)$, onde $Z$ é um novo símbolo do tipo $\tau \Leftrightarrow$ (por hipótese de indução) para toda atribuição $s$ em $M^{H}$ $\left\langle M, f^{-1}\left(s\left(X^{\tau}\right)\right)\right\rangle \vDash \psi\left(X^{\tau} \mid Z\right)^{T} \Leftrightarrow\langle M, a\rangle \vDash \psi\left(X^{\tau} \mid Z\right)^{T}$ para todo $a \in D_{\tau}^{M}$ (já que $f_{\tau}: D_{\tau}^{M} \rightarrow$ $M_{\tau}$ é uma bijeção e $\left.s\left(X^{\tau}\right) \in M_{\tau}\right) \Leftrightarrow M \vDash \psi^{T}\left[s^{\prime}\right]$ para toda atribuição $s^{\prime}$ em $M$ tal que $s^{\prime}\left(X^{\tau}\right) \in D_{\tau}^{M} \Leftrightarrow M \vDash \forall x\left(D_{\tau}(x) \rightarrow \psi^{T}\right) \Leftrightarrow M \vDash\left(\forall X^{\tau} \psi\right)^{T}$.

Agora, as condições (1) a (4) da Proposição 3.3.7 podem ser traduzidas em fórmulas de primeira ordem, isto é, existe um conjunto $\Theta$ de fórmulas de de primeira ordem tal que $M \vDash \Theta \Leftrightarrow M$ satisfaz (1) a (4). A condição (5) não pode pois equivale a uma fórmula infinitária $\forall x \bigvee_{\tau} D_{\tau}(x)$ (Hodges (1993)); contudo, vamos poder provar o seguinte: uma fórmula $\varphi$ é válida em todos os modelos $M^{H}$ se, e somente se, $\Theta \vdash \varphi^{T}$. Dessa forma, a validade de fórmulas de $L \omega$ nas estruturas $M^{H}$ é redutível à dedutibilidade de uma certa fórmula (obtida recursivamente) de uma teoria (recursiva) de primeira ordem.

Teorema 3.3.8 Considere o conjunto $\Theta$ formado pelas fórmulas de primeira ordem a seguir, correspondentes às condições (1) a (4) da Proposição 3.3.7:

(1) $D_{\tau}(v)$, para todo tipo $\tau$ e para todo simbolo $v$ de tipo $\tau$;

(2) $\exists x D_{\tau}(x)$, para todo tipo $\tau$;

(3) $\forall x\left(D_{\tau}(x) \rightarrow \neg D_{\tau^{\prime}}(x)\right)$, para todos os tipos $\tau, \tau^{\prime}, \tau \neq \tau^{\prime}$ e

(4) $\forall x, x_{1}, \ldots, x_{k}\left(\varepsilon_{\tau}\left(x, x_{1}, \ldots, x_{k}\right) \rightarrow D_{\tau}(x) \wedge D_{\tau_{1}}\left(x_{1}\right) \wedge \ldots \wedge D_{\tau_{k}}\left(x_{k}\right)\right)$, para todo tipo $\tau=\left(\tau_{1}, \ldots, \tau_{k}\right), k \geq 1$.

Então, para toda $\varphi \in L \omega(V)$,

$$
\vDash_{p H} \varphi \Leftrightarrow \Theta \vdash_{L 1} \varphi^{T},
$$

onde $\vDash_{p H}$ é a satisfação em pré-estruturas de Henkin.

Prova. Suponha que para $\vDash_{p H} \varphi$ e seja $M$ uma estrutura tal que $M \vDash \Theta$. Tome a subestrutura $M^{\prime}$ de $M$ tal que $M^{\prime}=\bigcup_{\tau} D_{\tau}^{M}$; então $M^{\prime} \vDash \Theta$ e, além disso, $M^{\prime}$ satisfaz a condição (5) da Proposição 3.3.7; assim, $M^{\prime} \vDash \varphi^{T}$. Vamos provar que para toda sentença $\varphi \in L \omega$, para todos $M$ e $M^{\prime}$ como acima,

$$
M^{\prime} \vDash \varphi^{T} \Rightarrow M \vDash \varphi^{T}
$$


Se $\varphi$ é atômica, então é da forma $v\left(v_{1}, \ldots, v_{k}\right)$, com $v, v_{1}, \ldots, v_{k}$ símbolos do tipo $\tau=$ $\left(\tau_{1}, \ldots, \tau_{k}\right), \tau_{1}, \ldots, \tau_{k}$, respectivamente, e assim $\varphi^{T}$ é $\varepsilon_{\tau}\left(v, v_{1}, \ldots, v_{k}\right)$. Logo $M^{\prime} \vDash \varphi^{T} \Leftrightarrow$ $M^{\prime} \vDash \varepsilon_{\tau}\left(v, v_{1}, \ldots, v_{k}\right) \Leftrightarrow\left\langle v^{M^{\prime}}, v_{1}^{M^{\prime}}, \ldots, v_{k}^{M^{\prime}}\right\rangle \in \varepsilon_{\tau}^{M^{\prime}}$. Agora, como $M^{\prime}$ é subestrutura de $M$, a interpretação dos símbolos em $M^{\prime}$ é a mesma que em $M$, donde $\left\langle v^{M^{\prime}}, v_{1}^{M^{\prime}}, \ldots, v_{k}^{M^{\prime}}\right\rangle=$ $\left\langle v^{M}, v_{1}^{M}, \ldots, v_{k}^{M}\right\rangle$, e além disso como $\varepsilon_{\tau}^{M^{\prime}}=\varepsilon_{\tau}^{M} \cap\left(M^{\prime}\right)^{k+1}$ (por definição), temos $\left\langle v^{M}, v_{1}^{M}, \ldots\right.$, $\left.v_{k}^{M}\right\rangle \in \varepsilon_{\tau}^{M}$, isto é, $M \vDash \varepsilon_{\tau}\left(v, v_{1}, \ldots, v_{k}\right)$.

O caso dos conectivos proposicionais segue diretamente da hipótese de indução. Suponha então que $\varphi$ é da forma $\forall x \psi$, onde $x$ é do tipo $\tau$; neste caso $\varphi^{T}$ é $\forall x\left(D_{\tau}(x) \rightarrow \psi^{T}\right)$. Assim, por hipótese, temos que para toda atribuição $s$ tal que $s(x) \in D_{\tau}^{M^{\prime}}$, vale $M^{\prime} \vDash \psi^{T}[s]$. Mas isto equivale a dizer que dado um novo símbolo de constante c, $\left\langle M^{\prime}, c^{M^{\prime}}\right\rangle \vDash \psi(x \mid c)^{T}$, onde $c^{M^{\prime}}$ é arbitrário em $D_{\tau}^{M^{\prime}}$; como $D_{\tau}^{M}=D_{\tau}^{M^{\prime}}$ por hipótese, temos que isto é equivalente a $\left\langle M, c^{M}\right\rangle \vDash \psi(x \mid c)^{T}$, onde $c^{M}$ é arbitrário em $D_{\tau}^{M}$, que equivale a $M \vDash \psi^{T}[s]$ para toda atribuição $s$ tal que $s(x) \in D_{\tau}^{M}$, que equivale a $M \vDash \forall x\left(D_{\tau}(x) \rightarrow \psi^{T}\right)$, isto é, $M \vDash(\forall x \psi)^{T}$.

Agora, $M$ era uma estrutra arbitrária tal que $M \vDash \Theta$, e asim provamos que $\Theta \vDash \varphi^{T}$. Por completude, $\Theta \vdash \varphi^{T}$.

Vamos ver agora como a validade de uma sentença de $L \omega$ na semântica padrão pode ser reduzida à validade de uma sentença de primeira ordem nos modelos de uma teoria de segunda ordem.

Teorema 3.3.9 Considere, para cada tipo $\tau=\left(\tau_{1}, \ldots, \tau_{k}\right), k \geq 1$, a fórmula de segunda ordem $\rho_{\tau}$ que expressa a representabilidade das relações sobre $D_{\tau_{1}}, \ldots, D_{\tau_{k}}$ por um objeto em $D_{\tau}$ :

$$
\begin{gathered}
\rho_{\tau} \leftrightarrow\left[\forall X \exists x \left(D _ { \tau } ( x ) \wedge \forall x _ { 1 } \ldots x _ { k } \left(D_{\tau_{1}}\left(x_{1}\right) \wedge \ldots \wedge D_{\tau_{k}}\left(x_{k}\right) \rightarrow\right.\right.\right. \\
\left.\left.\left.\left(X\left(x_{1}, \ldots, x_{k}\right) \leftrightarrow \varepsilon_{\tau}\left(x, x_{1}, \ldots, x_{k}\right)\right)\right)\right)\right] .
\end{gathered}
$$

Então, para toda sentença $\varphi \in L \omega$, temos que

$$
\vDash \varphi \Leftrightarrow \Theta \cup\left\{\rho_{\tau}\right\}_{\tau \in \mathcal{T}} \vDash \varphi^{T}
$$

na semântica padrão.

Prova. Basta observar que os modelos da semântica padrão são pré-estruturas de Henkin onde $M_{\tau}=P\left(M_{\tau_{1}} \times \ldots \times M_{\tau_{k}}\right)$ e que as fórmulas $\rho_{\tau}$ garantem a existência de todos os elementos necessários em $D_{\tau}$.

Corolário 3.3.10 Uma sentença $\varphi \in L \omega(V)$ é válida na semântica padrão se, e somente se, para uma certa fórmula $\chi_{\varphi}$ de $L 2\left(V^{T}\right)$ a fórmula $\chi_{\varphi} \rightarrow \varphi^{T}$ é válida na semântica padrão.

Prova. Veja que a existência das relações necessárias para a validade de $\varphi^{T}$ na semântica padrão depende apenas de uma quantidade finita de fórmulas $\rho_{\tau}$; tome então $\chi_{\varphi}$ como a conjunção destas fórmulas.

Encerraremos a sessão com um corolário interessante deste teorema, que ajuda a ter uma noção da complexidade do conjunto das fórmulas universalmente válidadas de $L 2$ :

Teorema 3.3.11 O conjunto (dos números de Gödel) das sentenças de segunda ordem válidas na semântica padrão não é definivel em $N$, a estrutura dos números naturais, por nenhuma fórmula da Lógica de Ordem Finita, isto é, não existe $\sigma \in L \omega$ com uma única variável livre $x$ tal que para toda $\varphi \in L 2$ 


$$
\vDash \varphi \Leftrightarrow N \vDash \sigma[\# \varphi]
$$

Prova. Suponha que exista uma tal $\sigma$. Seja $f$ um novo símbolo de função tal que $f^{N}: N \rightarrow N$ é a função primitiva recursiva tal que $f^{N}(\# \psi)=\#\left(\chi_{\psi} \rightarrow \psi^{T}\right)$, e seja $\Delta$ uma conjunção de fórmulas que definem $f$. Agora, pelo teorema 1.2.6 e pelo corolário 3.3.10,

$$
N \vDash \psi \Leftrightarrow \vDash P 2 \rightarrow \psi \Leftrightarrow N \vDash \sigma\left[f^{N}(\#(P 2 \rightarrow \psi))\right],
$$

e assim

$$
N \vDash \psi \Leftrightarrow N \vDash \Delta^{\prime} \rightarrow \sigma(f(\operatorname{impl}(\overline{\# P 2}, x)))[\# \psi],
$$

onde $\Delta^{\prime}$ é a conjunção das fórmulas em $\Delta$ mais a definição da função $i m p l(x, y)$ (que fornece o número de Gödel de $A \rightarrow B)$ e $\overline{\# 2}$ é o numeral de \#P2, isto é, é o símbolo da função sucessor aplicada \#P2 vezes. Mas então obtemos uma definição de verdade de $L \omega$ para $L \omega$ sobre $N$, contrariando o teorema 3.3.2. 


\section{Capítulo 4}

\section{Considerações Finais}

Em Chang e Keisler (1992), há uma lista de problemas em aberto sugerida pelos autores para o desenvolvimento da Teoria de Modelos. O último item da lista é "Desenvolver a Teoria de Modelos da Lógica de Segunda Ordem". Ao longo de nosso trabalho, estivemos investigando esta lógica, e alguns resultados puderam ser observados.

Frisamos que ao definir-se uma Lógica, é preciso dar a classe de estruturas nas quais queremos que as fórmulas, ou sentenças da lógica em questão sejam interpretadas. Com essa liberdade de escolha, notamos que a classe de estruturas introduzida por Henkin em Henkin (1950) permite uma teoria de modelos que podemos dizer, em certo sentido, que é tão rica quanto a teoria de modelos da Lógica de Primeira Ordem. Pudemos observar também que a teoria de conjuntos de segunda ordem introduzida no capítulo $2, Z F 2$, tem uma teoria de modelos suficientemente semelhante à de $Z F C$ para que se possa construir uma relação de forcing em $Z F 2$ e assim se obter vários resultados de independência. Em particular, a Hipótese do Contínuo não pode ser decidida a partir de $Z F 2$.

No entanto, o problema da Teoria de Modelos que Chang e Keisler (1992) se refere é com relação à semântica padrão, e não à semântica de Henkin. Como visto, esta semântica falha em cumprir várias propriedades da Lógica de Primeira Ordem, e propriedades que são fundamentais para as principais construções e teoremas conhecidos da Lógica de Primeira Ordem (Hodges (1993)). Isso dificulta, é claro, a contrução de um corpo de teoremas e resultados mais uniforme, para não mencionar que dificulta a obtenção mesmo de qualquer resultado. Pesquisando ainda a literatura sobre o assunto, nós achamos pouca coisa, tais como os resultados sobre o número de Hanf de L2 (sobre os quais nos detivemos no capítulo 3). Um resultado que nos pareceu interessante, mas que desconfiamos da demonstração em alguns momentos e não fomos mais à fundo, foi o de Ajtai (1979), que diz que a noção de isomorfismo e $L$-equivalência podem ser equivalentes, dependendo da meteoria em questão (basicamente, o argumento é que se $V=L$, então serão equivalentes; para construir um universo onde não são equivalentes, usa-se reais de Cohen).

Contudo, queremos mencionar que Barwise e Feferman (1985) nos pareceu particularmente otimista quanto ao desenvolvimento da Teoria de Modelos da semântica padrão de $L 2$, e traz um resultado devido a Shelah sobre quantificadores definíveis.

Um aspecto que não abordamos nesse trabalho, e que no entanto nos parece interessante, é o de ser menos exigente quanto à definição de sistema dedutivo. À nossa mente vem que, talvez, se exigíssemos um pouco menos sobre os sistemas dedutivos (e esse menos só Deus sabe o que seria) conseguiríamos mais recursos para a construção de modelos de teorias, e assim alguns resultados mais sistemáticos. Para uma certa lógica infinitária, por exemplo, foi possível fornecer um teorema de completude, uma vez abandonada a noção de conjuntos recursivamente enumeráveis (Barwise e Feferman (1985)). Mas na nossa pesquisa 
não achamos material sobre o assunto e, sinceramente, nem sabemos por onde começar.

Para não encerrar num tom tão pessimista, acentuamos o fato de que, apesar de que a semântica de Henkin não ser a semântica que muitos de nós gostaríamos, ela tem a sua utilidade, e quem sabe investigando-a mais a fundo, talvez possamos obter mais luz sobre a semântica padrão. O fato de que podemos adicionar axiomas lógicos aos nossos sistemas e continuar obtendo completude é, a nosso ver, motivador, e a falta de sugestões para a adição de axiomas é um tanto intrigante; esse é um problema onde nos perguntamos "afinal de contas, quais são os princípios lógicos necessários para se reger a Lógica das propriedades?", ou ainda, talvez de uma maneira ainda mais perplexa e aventureira: "afinal de contas, o que é Lógica de Segunda Ordem?" 


\section{Referências Bibliográficas}

Ajtai (1979) M. Ajtai. Isomorphism and higher order equivalence. Annals os Mathematical Logic, 16 (3):181-203. Citado na pág. 79

Barwise e Feferman (1985) J. Barwise e S. Feferman. Model-Theoretic Logics. SpringerVerlag. Citado na pág. 6, 79

Barwise (1972) K. J. Barwise. The hanf number of second order logic. The Journal of Symbolic Logic, 37:558-594. Citado na pág. 66

Chang e Keisler (1992) C. C. Chang e H. J. Keisler. Model Theory. Elsevier, $3^{\mathrm{a}}$ ed. Citado na pág. 6,79

Chuaqui (1972) R. Chuaqui. Forcing for the impredicative theory of classes. The Journal of Symbolic Logic, 37:1-18. Citado na pág. 32, 50, 51, 53

Cohen (2008) P. Cohen. Set Theory and The Continuum Hypothesis. Dover. Citado na pág. 32

Enderton (2000) H. Enderton. A Mathematical Introduction to Logic. Academic Press Pod, $2^{\text {a }}$ ed. Citado na pág. $6,8,10,11,22,71$

Fraenkel et al. (1973) A. Fraenkel, Y. Bar-Hillel e A. Levy. Foundations of Set Theory. North-Holland, $2^{\text {a }}$ ed. Citado na pág. 21, 66

Gallin (1975) D. Gallin. Intensional and higher-order modal logic: with applications to Montague semantics. North-Holland. Citado na pág. 17

Henkin (1950) L. Henkin. Completeness in the theory of types. The Journal of Symbolic Logic, 15:81-91. Citado na pág. iii, v, 14, 17, 79

Henkin (1949) L. Henkin. The completeness of the first-order functional calculus. The Journal of Symbolic Logic, 14:159-166. Citado na pág. 17

Hodges (1993) W. Hodges. Model Theory. Cambridge University Press. Citado na pág. 26, $64,75,79$

Jech (2006) T. Jech. Set Theory. Springer, $3^{\text {a }}$ ed. Citado na pág. 45, 62

Kreisel (1968) G. Kreisel. A survey of proof theory. The Journal of Symbolic Logic, 33: 321-388. Citado na pág. 23

Kreisel (1967) G. Kreisel. Comentários num artigo de mostowski. Em I. Lakatos, editor, The Philosophy of Mathematics, páginas 97-102. Elsevier. Citado na pág. 25 
Lindström (1969) P. Lindström. On extensions of elementary logic. Theoria, 35:1-11. Citado na pág. 64

Lévy (1965) A. Lévy. A hierarchy of formulas in set theory. Memoirs of the American Mathematical Society, 57. Citado na pág. 69

Montague (1965) R. Montague. Set theory and higher order logic. Em J. Crossley e M. Dummett, editors, Formal Systems and Recursive Functions, páginas 131-148. NorthHolland. Citado na pág. 13

Moore (1980) G. H. Moore. Beyond first-order logic: The historical interplay between mathematical logic and axiomatic set theory. History and Philosophy of Logic, 1:95-137. Citado na pág. 1

Shapiro (2000) S. Shapiro. Foundations without Foundationalism: A Case for Second-order Logic. Oxford University Press. Citado na pág. 7

Shepherdson (1952) J. Shepherdson. Inner models for set theory - part ii. The Journal of Symbolic Logic, 16:225-237. Citado na pág. 26

Shoenfield (1967) J. Shoenfield. Mathematical Logic. Addison-Wesley Pub. Co. Citado na pág. 27, 29, 30, 31, 44

Van Benthem e Doets (1983) J. Van Benthem e K. Doets. Higher-order logic. Em D. Gabbay e F. Guenthner, editors, Handbook of philosophical logic, volume 1, páginas 275-329. Reidel. Citado na pág. 25

Weston (1976) T. Weston. Kreisel, the continuum hypothesis and second order set theory. Journal of Philosophical Logic, 5:281-298. Citado na pág. 25

Weston (1974) T. Weston. The Continuum Hypothesis: Independende and Truth-Value. Tese de Doutorado, Massachussets Institute of Technology. Citado na pág. 25

Zermelo (1930) E. Zermelo. Über grenzzahlen und mengenbereiche. Fundamenta Mathematicae, 16:29-47. Citado na pág. iii, v, 26 